MPP-2008-144

DIAS-STP 08-15

Bonn-TH 2008-15

SLAC-PUB-13466 revised 12/2008

\title{
GUTs in Type IIB Orientifold Compactifications
}

\author{
Ralph Blumenhagen ${ }^{1}$, Volker Braun ${ }^{2}$, Thomas W. Grimm ${ }^{3}$, and Timo Weigand ${ }^{4}$ \\ ${ }^{1}$ Max-Planck-Institut für Physik, Föhringer Ring 6, \\ 80805 München, Germany \\ ${ }^{2}$ Dublin Institute for Advanced Studies, 10 Burlington Road, Dublin 4, Ireland \\ ${ }^{3}$ Bethe Center for Theoretical Physics and \\ Physikalisches Institut der Universität Bonn, Nussallee 12, \\ 53115 Bonn, Germany \\ 4 SLAC National Accelerator Laboratory, Stanford University, \\ 2575 Sand Hill Road, Menlo Park, CA 94025, USA \\ blumenha@mppmu.mpg.de, vbraun@stp.dias.ie, \\ grimm@th.physik.uni-bonn.de, timo@slac.stanford.edu
}

\begin{abstract}
We systematically analyse globally consistent $S U(5)$ GUT models on intersecting D7-branes in genuine Calabi-Yau orientifolds with O3- and O7-planes. Beyond the well-known tadpole and K-theory cancellation conditions there exist a number of additional subtle but quite restrictive constraints. For the realisation of $S U(5)$ GUTs with gauge symmetry breaking via $U(1)_{Y}$ flux we present two classes of suitable Calabi-Yau manifolds defined via del Pezzo transitions of the elliptically fibred hypersurface $\mathbb{P}_{1,1,1,6,9}[18]$ and of the Quintic $\mathbb{P}_{1,1,1,1,1}[5]$, respectively. To define an orientifold projection we classify all involutions on del Pezzo surfaces. We work out the model building prospects of these geometries and present five globally consistent string GUT models in detail, including a 3-generation $S U(5)$ model with no exotics whatsoever. We also realise other phenomenological features such as the $10105_{\mathbf{H}}$ Yukawa coupling and comment on the possibility of moduli stabilisation, where we find an entire new set of so-called swiss-cheese type Calabi-Yau manifolds. It is expected that both the general constrained structure and the concrete models lift to F-theory vacua on compact Calabi-Yau fourfolds.
\end{abstract}




\section{Contents}

\begin{tabular}{lll}
\hline & Introduction & 4
\end{tabular}

2 Orientifolds with Intersecting D7-Branes 10

2.1 Intersecting D7-Branes With Gauge Bundles . . . . . . . . . . . . . . 11

2.2 Tadpole Cancellation for Intersecting D7-Branes . . . . . . . . . . . . 16

2.3 The Massless Spectrum . . . . . . . . . . . . . . . . . . . . . . . . . 20

2.3 .1 Matter Divisors . . . . . . . . . . . . . . . . 21

2.3 .2 Matter Curves . . . . . . . . . . . . . . . . . . 22

2.4 F- and D-Term Supersymmetry Constraints . . . . . . . . . . 23

$\begin{array}{llll}3 & \text { SU(5) GUTs and Their Breaking } & 25\end{array}$

3.1 Georgi-Glashow SU(5) GUT . . . . . . . . . . . . . . . . . . . . 26

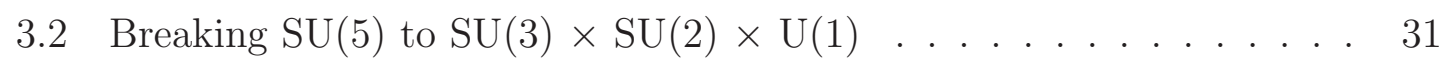

3.3 Summary of GUT Model Building Constraints . . . . . . . . . . . . . 37

$4 \quad$ Del Pezzo Transitions on $\mathbb{P}_{1,1,6,0}[18]$

4.1 Del Pezzo Surfaces and Their Involutions . . . . . . . . . . . . . . . . 39

4.2 The Geometry of Del Pezzo Transitions of $\mathbb{P}_{1,1,6,9}[18]$. . . . . . . . . 43

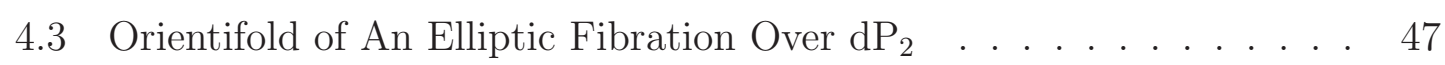

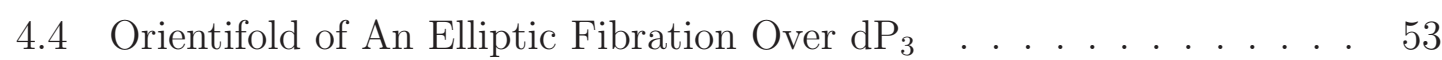

4.5 The Swiss-Cheese Property . . . . . . . . . . . . . . . . . . . 56

4.6 D-Term Conditions For D7-Branes on Del Pezzo Surfaces . . . . . . . 58

$\begin{array}{lll}5 & \text { A GUT Model on } \mathrm{M}_{2}^{\left(\mathrm{dP}_{9}\right)^{2}} & 60\end{array}$

5.1 The Chiral Model . . . . . . . . . . . . . . . . . . . . . . 61

5.2 D3-Brane Tadpole and K-Theory Constraints . . . . . . . . . . . 62

5.3 D-Flatness . . . . . . . . . . . . . . . . . . . . 64

5.4 Globally Consistent Model . . . . . . . . . . . . . . . . 65

$6 \quad$ GUT Model Search $\quad 73$

$6.1 \quad$ A 3-Generation GUT Model on $M_{2}^{\left(\mathrm{dP}_{8}\right)^{2}} \ldots \ldots \ldots$. . . . . . . . 73 
$6.2 \quad$ A GUT Model on $M_{3}^{\left(\mathrm{dP}_{9}\right)^{3}} \ldots \ldots \ldots \ldots$. . . . . . . . . 80

7 GUTs on Del Pezzo Transitions of the Quintic 85

7.1 Del Pezzo Transitions of the Quintid . . . . . . . . . . . . . . . . 87

7.2 A GUT Model Without Vector-Like Matter . . . . . . . . . . . . . . 92

7.3 A Three-Generation Model With Localised Matter on $Q^{\left(\mathrm{dP}_{9}\right)^{4}}$. . . . . 96

8 Comments on Moduli Stabilisation 98

$\begin{array}{lll}9 & \text { Conclusions } & 101\end{array}$

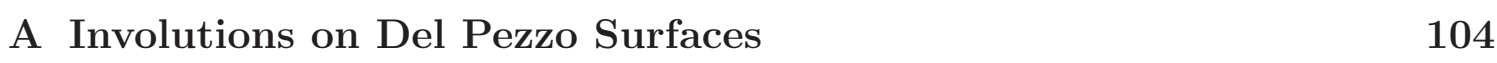

A.1 Del Pezzo Surfaces of High Degree . . . . . . . . . . . . . . . . . . . . 104

A.2 Involutions on the Projective Plane . . . . . . . . . . . . . . . . . . . 104

A.3 Involutions on the Product of Lines . . . . . . . . . . . . . . . 106

A.4 Blow-up of the Projective Plane . . . . . . . . . . . . . . . . . . . . . 107

A.5 Blow-up of the Projective Plane at Two Points . . . . . . . . . . . . . . 109

A.6 Blow-up of the Projective Plane at Three Points . . . . . . . . . . . . . 112

A.7 The Weyl Group and The Graph of Lines . . . . . . . . . . . . . . . . 115

A.8 Minimal Involutions . . . . . . . . . . . . . . . . . . . . . . . . 120

A.9 Blow-ups of Minimal Models . . . . . . . . . . . . . . . . . . . . 122

A.10 Explicit Realisations . . . . . . . . . . . . . . . . . . . 124

B Cohomology of Line Bundles over del Pezzo Surfaces 126

\begin{tabular}{lll}
\hline C Cohomology of Line Bundles On Rational Elliptic Surfaces & 129
\end{tabular}

\begin{tabular}{lr}
\hline Bibliography & 131
\end{tabular} 


\section{Introduction}

The LHC experiment is widely expected not only to confirm the existence of the Higgs particle as the last missing ingredient of the Standard Model of Particle Physics, but also to reveal new structures going far beyond. As experiments are proceeding into this hitherto unexplored energy regime, string theory, with its claim to represent the unified theory of all interactions, will have to render an account of its predictions for physics beyond the Standard Model. Clearly, these depend largely on the value of the string scale $M_{s}$, the most dramatic outcome corresponding to $M_{s}$ close to the $\mathrm{TeV}$ scale. While this is indeed a fascinating possibility, in concrete string models it often leads to severe cosmological issues such as the cosmological moduli problem. In this light it might be fair to say that a more natural (but also more conservative) scenario involves a value of $M_{s}$ at the GUT, Planck or intermediate scale.

During the last years, various classes of four-dimensional string compactifications with $\mathcal{N}=1$ spacetime supersymmetry have been studied in quite some detail (see the reviews [1, 2, 3, 4, 5, for references). From the viewpoint of realising the Minimal Supersymmetric Standard Model (MSSM) and some extension thereof the best understood such constructions are certainly the perturbative heterotic string and Type IIA orientifolds with intersecting D6-branes. On the contrary, as far as moduli stabilisation is concerned Type IIB orientifolds with O7- and O3-planes look very promising. The combination of three-form fluxes and D3-brane instantons can stabilise all closed string moduli [6] even within the solid framework of (conformal) Calabi-Yau manifolds where reliable computations can be performed. Moreover, supersymmetry breaking via Kähler moduli mediation and the resulting structure of soft terms bear some attractive features and have been studied both for the LARGE volume scenario [7,8] with an intermediate string scale and for a GUT scenario with the string scale at the GUT scale [9, 10].

These considerations are reason enough to seriously pursue model building within type IIB orientifolds. The observation that the MSSM gauge couplings appear to meet at the GUT scale furthermore suggests the existence of some GUT theory at high energies. GUT gauge groups such as $S U(5)$ and $S O(10)$ appear naturally in string theories based on gauge group $E_{8}$ like the heterotic string. On the other hand, it has become clear that for perturbative orientifolds with D-branes, exceptional gauge groups and features like the spinor representations of $S O(10)$ do not emerge. For $S U(5)$ D-brane models, by contrast, the gauge symmetry and the desired chiral matter spectrum can be realised, a fact welcome in view of the described progress in 
Type IIB moduli stabilisation. Still, at first sight there appears a serious problem in the Yukawa coupling sector. The $\mathbf{1 0 1 0} \mathbf{5}_{\mathbf{H}}$ Yukawa coupling violates global perturbative $U(1)$ symmetries which are the remnants of former $U(1)$ symmetries rendered massive by the Stückelberg mechanism [11]. As a consequence of these considerations it is sometimes argued that the natural context for Type II GUT model building is the strong coupling limit, where the crucial couplings in question are not "perturbatively" forbidden. The strongly coupled duals of type IIA and Type IIB orientifolds are given by singular M-theory compactifications on $G_{2}$ manifolds and, respectively, by F-theory compactifications on elliptically fibred Calabi-Yau fourfolds [12. The local model building rules for such F-theory compactifications have been worked out recently in [13, 14, 15, 16, 17, 18, 19]; For recent studies of 7-branes from the F-theory perspective see [20,21, 22, 23, 24].

On the other hand, investigations of non-perturbative corrections for Type II orientifold models [25, 26, 27] have revealed that the $10105_{\mathbf{H}}$ Yukawa coupling can be generated by Euclidean D-brane instantons wrapping suitable cycles $\Gamma$ in the internal manifold with the right zero mode structure [28]. Of course these couplings are suppressed 1 by the exponential of the instanton action $\Re\left(T_{\text {inst }}\right)=g_{s}^{-1} \operatorname{Vol}(\Gamma)$. It is crucial to appreciate that this suppression is not tied to the inverse gauge coupling of the Standard Model, as would be the case for effects related to gauge (as opposed to "stringy") instantons, but can in principle take any value, depending on the geometric details of our compactification manifold. This feature, which holds both for Type IIA and Type IIB orientifolds, opens up the prospect of $S U(5)$ GUT model building already in the limit $T_{\text {inst }} \rightarrow 0$ of perturbative Type II orientifolds. Once we also take the nice features of moduli stabilisation in Type IIB into account, one might seriously hope that the strong coupling limit of Type IIB orientifolds, either in their genuinely F-theoretic disguise or in their perturbative description as D-brane models with $\mathrm{O} 7$ - and $\mathrm{O} 3$ - planes and $T_{\text {inst }} \rightarrow 0$, may indeed provide a promising starting point to construct realistic GUT models.

In the recent work [14, 16], the authors draw the first conclusion (see also [13, 17]). Taking into account that F-theory models on elliptically fibred fourfolds can admit degenerations of the elliptic fibre such that exceptional gauge groups appear naturally, these references pursue the program of studying GUT type F-theory compactifications. As a physical input, the authors of [14,16] propose the working hypothesis

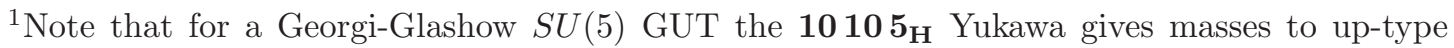
quarks, whereas for flipped $S U(5)$ it provides the down-type quark masses. In the latter case, the exponential suppression might explain the little hierarchy between up and down quarks 28].
} 
that the Planck scale ought to be decouple-able from the GUT scale, even if only in principle. From the Type IIB perspective, these F-theoretic models do not only contain usual D-branes, but also so-called $(p, q)$ seven-branes which carry charge under both the R-R and the NS-NS eight-forms. The new non-perturbative states, such as the gauge bosons of exceptional groups or the spinor representations of $S O(10)$, are given by $(p, q)$ string junctions starting and ending on these branes. Unlike fundamental strings, these string junctions can have more than two ends thus providing extra states.

From the guiding principle of decoupled gravity it is further argued in [16] that the $(p, q)$ 7-branes should wrap shrinkable four-cycles in the internal geometry. These are given by del Pezzo surfaces2. Lacking a global description of Calabi-Yau fourfolds with the desirable degeneration, the authors provide a local set-up of singularities or $(p, q)$ 7-branes and line bundles so that the GUT particle spectrum is realised. At a technical level there arises a challenge with GUT symmetry breaking because a theory on a del Pezzo surface has neither adjoints of $S U(5)$ nor discrete Wilson lines at its disposal to break $S U(5)$ to $S U(3) \times S U(2) \times U(1)_{Y}$. One option would be to adopt the philosophy of heterotic compactifications and embed a further non-trivial $U(1)$ line bundle, as discussed for the heterotic string originally in [31] and more recently in [32,33,34,35]. For line bundles non-trivial on the Calabi-Yau manifold, the associated $U(1)$ generically becomes massive due to the Stückelberg mechanism, but in presence of several line bundles special linear $U(1)$ combinations remain massless. In the heterotic context of [34, in order to maintain gauge coupling unification without relying on large threshold corrections it is necessary to consider the large $g_{s}$ limit of heterotic M-theory [36].

As a new and very central ingredient the authors of [16] propose to break the GUT gauge group instead by a line bundle embedded into $U(1)_{Y}$ such that it circumvents the sort of no-go theorem mentioned above (see also [17]). The idea is to support the bundle on a non-trivial two-cycle inside the del Pezzo surface which is trivial on the ambient four-fold base. It was argued in [16] that with this mechanism some of the notorious problems of GUTs such as the doublet-triplet splitting problem, dangerous dimension five proton decay operators and even neutrino masses can be addressed and actually solved by appropriate choices of matter localisations and line bundles on the del Pezzo divisors. Studies of supersymmetry breaking mechanisms for this class of local models have appeared in [37, 38, 39].

The Planck-scale decoupling principle might be a justification for a local approach

\footnotetext{
${ }^{2}$ Local quiver type models on del Pezzo singularities have been studied, for example, in [29, 30 .
} 
to string model building (and indeed a quite constraining one), but in absence of a realisation of the described mechanisms in globally consistent string compactifications it remains an open question if these local GUT models do really consistently couple to gravity. In fact, it is the global consistency conditions of string theory which decide whether a given construction is actually part of the string landscape or merely of the "swampland" of gauge theories. At a technical level, it is therefore no wonder that they constitute some of the biggest challenges in string model building, and many interesting local constructions fail to possess a compact embedding satisfying each of these stringy consistency conditions. For example, whether or not a given $U(1)_{Y}$ flux actually leads to a massless hypercharge depends on the global embedding of the divisor supporting the 7-brane into the ambient geometry and cannot be decided within a local context.

In F-theory the global consistency conditions, in particular the D7-brane tadpole cancellation condition, are geometrised: They are contained in the statement that indeed a compact elliptically fibred fourfold exists such that the degenerations of the fibre realise the GUT model. This is a very top-down condition and given the complexity and sheer number of fourfolds it is extremely hard to implement in practise.

It is the aim of this paper to address these global consistency conditions by taking a different route. As described above Georgi-Glashow SU(5) GUT models can naturally be realised on two-stacks of D7-branes in a perturbative Type IIB orientifold. Here we have quite good control over the global consistency conditions as they are very similar to the well-studied Type I or Type IIA orientifolds. Therefore, our approach is to first construct a GUT model on a Type IIB orientifold, satisfy all consistency conditions, check whether the top quark Yukawa is really generated by an appropriate D3-instanton and then take the local $T_{\text {inst. }} \rightarrow 0$ limit 3 .

To follow this path, we start by partly newly deriving, partly summarising the model building rules for Type IIB orientifolds. We then study how much of the appealing structure proposed in the F-theoretic context, such as the $U(1)_{Y}$ GUT gauge breaking, can already be realised in perturbative Type IIB orientifolds on Calabi-Yau threefolds with intersecting D7-branes wrapping holomorphic surfaces with non-trivial vector- or line-bundles.

In order for the $U(N)$ gauge factors on the D7-banes not to exhibit chiral mul-

\footnotetext{
${ }^{3}$ Here we make the working assumption that a Type IIB vacuum satisfying all K-theory and supersymmetry constraints has an uplift to F-theory on a Calabi-Yau fourfold. We are aware that this may not be so straightforward to show [40].
} 
tiplets transforming in the adjoint representation, the four-cycle wrapped by the 7-brane should be rigid. A natural class of such complex four-manifolds is again given by del Pezzo surfaces, which therefore remain important ingredients from a phenomenological point of view 4 . This leads us to the study of a class of suitable Calabi-Yau threefolds admitting complex divisors of del Pezzo type whose non-trivial two-cycles are partially trivial when considered as two-cycles of $Y$. A large class of such Calabi-Yau threefolds is given by del Pezzo transitions, as have been recently discussed in a slightly different context in [41. The idea is to start for instance with the well-studied "swiss cheese" type Calabi-Yau hypersurface $\mathbb{P}_{1,1,1,6,9}[18]$, which is elliptically fibred over $\mathbb{P}_{2}$, and blow up points on the base. As a second starting point for such del Pezzo transitions we consider the quintic $\mathbb{P}_{4}[5]$. As we will see such Calabi-Yau manifolds are natural candidates for GUT model building.

More concretely, in Section 2 we collect the string model building rules for Type IIB orientifolds with O7- and O3-planes. Since these involve intersecting D7-branes supporting non-trivial gauge bundles, the relevant structure is a combination of Type I and Type IIA orientifold features and as such is slightly more complicated and subtle. In particular, since the del Pezzo surfaces wrapped by the D7-branes are not Spin, one has to take into account the Freed-Witten anomaly [42] shifting the proper quantisation for the gauge fluxes. Moreover, for involutions with $h_{-}^{2}>0$ one encounters dynamical $B$-field moduli, which have to be appropriately dealt with.

In Section 3 we discuss how we can realise a D-brane sector supporting a GeorgiGlashow $S U(5)$ GUT model. In many respects this is very analogous to $S U(5)$ GUTs in intersecting D6-brane scenarios (for concrete examples see, for example, [43, 11, 44, 45, 46, 47, 48]). We show that the GUT symmetry breaking via $U(1)_{Y}$ flux can be realised in the perturbative Type IIB orientifold framework and provide the conditions under which this flux can solve the doublet-triplet splitting problem and the suppression of dimension five proton decay operators. By studying which quantities are affected by this flux we find that not only the gauge group is broken but also the non-chiral spectrum and the D3-brane tadpole generically changes. Very analogous features are expected to arise also in F-theory compactifications on compact Calabi-Yau fourfolds equipped with non-vanishing four-form flux.

The slightly more mathematical Section 4 defines a class of compact Calabi-Yau

\footnotetext{
${ }^{4}$ Note, however, that the class of rigid surfaces is larger than that of shrinkable, that is, del Pezzo, surfaces. For example, the surface $\mathrm{dP}_{9}$, which is rigid, but not contractible, is still an interesting candidate for a GUT D7-brane in this respect.

${ }^{5} \mathrm{An}$ analogous quantisation condition is expected to also arise for the four-form flux in F-theory compactifications on compact as well as on non-compact Calabi-Yau fourfolds.
} 
manifolds naturally containing del Pezzo surfaces. These compact manifolds have recently been considered in [41] and contain the kind of holomorphic surfaces allowing for the realisation of many of the GUT features we are interested in. They can be described as elliptic fibrations over del Pezzo surfaces $\mathrm{dP}_{n}, n=1, \ldots, 8$ and their various connected phases related via flop transitions. In the elliptic fibration itself, besides the $\mathrm{dP}_{n}$ basis, we find various $\mathrm{dP}_{9}$ surfaces, which via the flop transitions become $\mathrm{dP}_{8}$ surfaces or $\mathbb{P}_{2}$ surfaces with more than nine points blown up. In the course of this section, to define the orientifold actions we have to investigate the existence of appropriate involutions. In order not to interrupt the physics elaborations too much this rather technical though central discussion has mainly been shifted to appendix A. The mathematically interested reader is encouraged to consult this appendix for more details on the classification of involutions and the determination of the fixed point loci. As an important part of our analysis we will prove the "swiss cheese" structure of those del Pezzo transitions where the $\mathrm{dP}_{9}$ surfaces have all been flopped to $\mathrm{dP}_{8}$ surfaces. We will show that as a consequence of this structure the D-term supersymmetry conditions force the cycles supporting D-branes to take a vanishing volume, that is, they are dynamically driven to the quiver locus.

In Sections 5 and 6 we present some first concrete $S U(5)$ GUT models. These are the outcome of an essentially manual search which has succeeded in implementing all known global consistency conditions. As a warm-up, Section 5 discusses at length an $S U(5)$ model on the Weierstraß model over $\mathrm{dP}_{2}$ with two chiral families of $S U(5)$ GUT matter, one vector-like pair of Standard Model Higgs and no chiral exotics. The GUT matter transforming in the $\mathbf{1 0}$ is localised in the bulk of the GUT branes, while the $\overline{\mathbf{5}}$ and the Higgs pair arise at matter curves. Upon breaking $S U(5)$ by means of $U(1)_{Y}$ flux there arise extra vector-like pairs of Standard Model matter. As one of its phenomenologically appealing features, this model contains a $10105_{\mathbf{H}}$ Yukawa coupling of order one induced by a Euclidean D3-brane instanton in the limit $T_{\text {inst. }} \rightarrow 0$, but the global consistency conditions do not allow for the construction of a three-generation model on this particular geometry. To remedy this we present, in Section 6, a string vacuum of a similar type on the del Pezzo transition of this Weierstraß model, but featuring three chiral families of Standard Model matter, no chiral exotics and only two pairs of extra vector-like states. As a consequence of the swiss cheese structure of the manifold, the D-term supersymmetry conditions drive the vacuum to the boundary of the Kähler cone. This can be avoided in a three-generation GUT model on the Weierstraß model over $\mathrm{dP}_{3}$ as discussed in the remainder of this section. The key phenomenological achievements and drawbacks of these three examples are summarised in Tables 9, 13, and 15. 
In Section 7 we analyse yet another class of geometries based on the quintic hypersurface which also contain del Pezzo surfaces suitable for GUT model building by extending the examples of [41]. In this type of geometries it is possible to localise all GUT matter at matter curves, thus avoiding the appearance of extra vectorlike pairs in the bulk. The D-term supersymmetry conditions can be realised for non-zero cycle volume. Once again due to subtle global consistency constraints we only present a two-generation GUT model exhibiting these features realised on $\mathrm{dP}_{8}$ surfaces, but remarkably we do find an interesting model with just three generations of Standard Model matter and no exotics whatsoever on a related geometry featuring $\mathrm{dP}_{9}$ surfaces. The detailed properties of these two examples are summarised in Tables 17 and 19, respectively.

In Section 8 we comment on the possibilities of moduli stabilisation in our class of models. Taking our findings for GUT model building into account, the most natural realisation of the LARGE volume scenario of moduli stabilisation seems to place the GUT branes and the instantons contributing to the superpotential on different, nonintersecting $\mathrm{dP}_{n}$ surfaces. This is expected to lead to a new pattern of soft terms at the GUT scale and consequently to different collider signatures compared to the studies which have appeared in the literature so far. Furthermore, we clearly need to stabilise the string scale not at an intermediate, but at the GUT scale, for example along the lines of [10]. The explicit elaboration of such aspects is, however, beyond the scope of the present article.

\section{Orientifolds with Intersecting D7-Branes}

In this section we will introduce some preliminaries on Type IIB Calabi-Yau orientifold compactifications with space-time filling D7 branes. In order for such a scenario to be globally consistent and to preserve $\mathcal{N}=1$ supersymmetry in the four flat dimensions we consider an orientifold projection which allows for $\mathrm{O} 3$ and $\mathrm{O} 7$ planes and takes the form $\mathcal{O}=(-1)^{F_{L}} \Omega_{p} \sigma$. Here $\sigma$ is a holomorphic and isometric involution of the internal Calabi-Yau manifold $Y$. The action of $\sigma$ on the Kähler form $J$ of $Y$ is $\sigma^{*} J=J$, while the holomorphic $(3,0)$ form transforms as $\sigma^{*} \Omega=-\Omega$. Similarly, for the other string fields to remain in the spectrum they have to transform with the appropriate parity under $\sigma$.

To determine the four-dimensional effective theory one first needs to examine the surviving bulk and brane fields. At least locally, each such field can be identified with an element of an appropriate bundle valued cohomology group on the internal mani- 
fold and the cycles wrapped by the D-branes. The involution $\sigma$ splits the cohomology groups into eigenspaces, and allows one to identify the spectrum preserved by the orientifold. Focusing on the bulk fields corresponding to closed string excitations, one notes that $H^{p, q}(Y, \mathbb{C})$ splits as $H_{+}^{p, q} \oplus H_{-}^{p, q}$ with dimensions $h_{ \pm}^{p, q}$ respectively. One thus obtains the complex dilaton $\tau=C_{0}+i e^{-\phi}, h_{+}^{1,1}$ complexified Kähler moduli $T_{I}$ and $h_{-}^{1,1}$ B-field moduli $G^{i}$ given by [49, 50]

$$
T_{I}=\int_{\Gamma_{I}^{+}} \Pi, \quad G^{i}=\int_{\gamma_{-}^{i}} \Pi, \quad \Pi=e^{-B}\left(e^{-\phi} \operatorname{Re}\left[e^{i J}\right]+i C_{\mathrm{RR}}\right)
$$

where $C_{\mathrm{RR}}=C_{0}+C_{2}+C_{4}$. The cycles $\Gamma_{I}^{+}$and $\gamma_{-}^{i}$ form a basis of the homology groups $H_{2,2}^{+}$and $H_{1,1}^{-}$, respectively. We will call the continuous moduli $G^{i}$ simply $B_{-}$ moduli, since they encode the variations of the NS-NS and R-R two-forms. While no dynamical moduli are associated with the reduction of the B-field along the positive 2 -cycles $\Gamma_{+}^{I} \in H_{1,1}^{+}$there can still be discrete non-zero B-flux $\frac{1}{2 \pi} \int_{\Gamma_{+}^{I}} B=\frac{1}{2}$. This survives the orientifold action due to the axionic shift symmetry $\int_{\Gamma_{+}^{I}} B \rightarrow \int_{\Gamma_{+}^{I}} B+2 \pi$ and will sometimes be referred to as $B_{+}$flux. In the following we will determine which quantities in the four-dimensional action depend on which of these closed string moduli.

\subsection{Intersecting D7-Branes With Gauge Bundles}

We first discuss the inclusion of space-time filling D7-branes in more detail. Consider wrapping a stack of $N_{a}$ D7-branes around a four-cycle $D_{a}$ in $Y$. The calibration condition for the D7-branes requires $D_{a}$ to be a holomorphic divisor [51]. The orientifold symmetry $\sigma$ maps $D_{a}$ to its orientifold image $D_{a}^{\prime}$ so that in the upstairs geometry each brane is accompanied by its image brane. There are three different cases to be distinguished:

1. $\left[D_{a}\right] \neq\left[D_{a}^{\prime}\right]$,

2. $\left[D_{a}\right]=\left[D_{a}^{\prime}\right]$ but $D_{a} \neq D_{a}^{\prime}$ point-wise, and

3. $D_{a}=D_{a}^{\prime}$ point-wise, that is, D7-branes coincide with an O-plane.

In the first two situations, the D7-brane may or may not intersect an O7-plane. For vanishing gauge flux, branes of the first type carry unitary gauge groups, while those of the other two types yield symplectic or orthogonal gauge groups. In absence of 
CFT methods to uniquely distinguish SO vs. SP Chan-Paton factors the rule of thumb is that a stack of $N_{a}$ branes plus their $N_{a}$ image branes on top of an $O 7^{-/+_{-}}$ plant 6 gives rise to a gauge group $S O\left(2 N_{a}\right) / S P\left(2 N_{a}\right)$. The same configuration along a cycle of type 2 with locally four Dirichlet-Neumann boundary conditions to the $O 7^{-/+}$-plane yields gauge group $S P\left(2 N_{a}\right) / S O\left(2 N_{a}\right)$.

\section{Gauge fluxes on D7-branes}

Each stack of D7-branes can carry non-vanishing background flux for the YangMills field strength $F_{a}$. Recall that the field strength $F_{a}$ appears in the ChernSimons and DBI action only in the gauge invariant combination $\mathcal{F}_{a}=F_{a}+\iota^{*} B \mathbf{1}$, where $\iota: D_{a} \rightarrow Y$ denotes the embedding of the divisor $D_{a}$ into the ambient space. Therefore all physical quantities depend a priori only on $\mathcal{F}_{a}$. However, as we will describe in detail below, with the exception of the D-term supersymmetry condition only the discrete $B_{+}$-flux effectively enters the consistency conditions.

A consistent configuration of internal gauge flux is mathematically described in terms of a stable holomorphic vector bundle 7 by identifying the curvature of its connection with the Yang-Mills field strength. For all concrete applications in this article it will be sufficient to restrict ourselves to the simplest case of line bundles $L_{a}$, corresponding to Abelian gauge flux. For a single D-brane wrapping a simply connected divisor these are determined uniquely by their first Chern class $c_{1}\left(L_{a}\right)$ as an element of $H^{2}\left(D_{a}\right)$ or equivalently by a two-cycle $l_{a}$ with class in $H_{2}\left(D_{a}\right)$ as $L_{a}=\mathcal{O}\left(l_{a}\right)$. For stacks of several coincident branes wrapping the divisor $D_{a}$ we also have to specify the embedding of the $U(1)$ structure group of the line bundle into the original gauge group on the branes.

Let us start with a stack of $N_{a}$ branes of type 1 and decompose the background value of the physical Yang-Mills field strength $\mathcal{F}$ as

$$
\mathcal{F}_{a}=T_{0}\left(F_{a}^{(0)}+\iota^{*} B\right)+\sum_{i} T_{i} F_{a}^{(i)} .
$$

Here $T_{0}=1_{N_{a} \times N_{a}}$ refers to the diagonal $U(1)_{a} \subset U\left(N_{a}\right)$ while $T_{i}$ are the traceless Abelian 8 elements of $S U\left(N_{a}\right)$. This defines the line bundles $L_{a}^{(i)}$ as

$$
c_{1}\left(L_{a}^{(0)}\right)=\frac{1}{2 \pi}\left(F_{a}^{(0)}+\iota^{*} B\right) \in H^{2}\left(D_{a}\right), \quad c_{1}\left(L_{a}^{(i)}\right)=\frac{1}{2 \pi} F_{a}^{(i)} \in H^{2}\left(D_{a}\right) .
$$

\footnotetext{
${ }^{6} \mathrm{~A} O 7^{-/+}$-plane carries $-8 /+8$ times the charge of a D7-brane in the upstairs geometry.

${ }^{7}$ More generally, gauge flux is described by coherent sheaves, but for our purposes it suffices to consider vector bundles on the divisors.

${ }^{8}$ This can be generalised to non-Abelian vector bundles. For example, on a stack of $N_{a}$ coincident
} 
Note that in view of the appearance of $\mathcal{F}_{a}$ in all physical equations the $B$-field is to be included in $c_{1}\left(L_{a}^{0}\right)$.

While the effect of $L_{a}^{(0)}$ is merely to split $U\left(N_{a}\right) \rightarrow S U\left(N_{a}\right) \times U(1)_{a}$, the other $L_{a}^{(i)}$ will break $S U\left(N_{a}\right)$ further. The relevant example we will be studying in detail is the breaking of $U(5)_{a} \rightarrow S U(5) \times U(1)_{a} \rightarrow S U(3) \times S U(2) \times U(1)_{Y} \times U(1)_{a}$ by means of diagonal flux and another line bundle corresponding to the hypercharge generator $T_{Y}$. Note that the Abelian gauge factors may become massive via the Stückelberg mechanism [54].

For a stack of $2 N_{a}$ invariant branes of type 2 and 3 a non-trivial bundle $L_{a}^{(0)}$ breaks $S O\left(2 N_{a}\right) / S P\left(2 N_{a}\right) \rightarrow S U\left(N_{a}\right) \times U(1)_{a}$ and the embedding of $L_{a}^{(i)}$ works in an analogous manner. We will be more specific in the context of the concrete setup described in Subsection 3.1.

In general it is possible for some components of $c_{1}\left(L_{a}\right)$ along $H^{2}\left(D_{a}\right)$ to be trivial as elements of $H^{2}(Y)$. Recall that the inclusion $\iota: D_{a} \rightarrow Y$ defines the pushforward $\iota_{*}: H_{2}\left(D_{a}\right) \rightarrow H_{2}(Y)$ and pullback $\iota^{*}: H^{2}(Y) \rightarrow H^{2}\left(D_{a}\right)$. Then one can split $L_{a}$ as

$$
L_{a}=\iota^{*} \mathbb{L}_{a} \otimes R_{a}
$$

with $\mathbb{L}_{a}=\mathcal{O}\left(\ell_{a}\right)$ defined as a line bundle on the Calabi-Yau $Y$. The part of $L_{a}$ trivial in $Y$, denoted as $R_{a}=\mathcal{O}\left(r_{a}\right)$, corresponds to a two-cycle $r_{a}$ which is non-trivial on $D_{a}$ but a boundary in $Y$, that is, $\left[r_{a}\right] \in \operatorname{ker}\left(\iota_{*}\right)$. The possibility of considering such gauge flux in the relative cohomology of $D_{a}$ in $Y$ was first pointed out in [55, 56] and its relevance for model building was stressed in [30,41].

We need to understand which quantities are affected by a relative flux $R$. In this context, we will make heavy use of the following integrals

$$
\begin{aligned}
& \int_{D_{a}} c_{1}\left(\iota^{*} \mathbb{L}_{b}\right) \wedge c_{1}\left(\iota^{*} \mathbb{L}_{c}\right)=\int_{Y}\left[D_{a}\right] \wedge c_{1}\left(\mathcal{O}\left(\ell_{b}\right)\right) \wedge c_{1}\left(\mathcal{O}\left(\ell_{c}\right)\right)=\kappa_{a b c} \\
& \int_{D_{a}} c_{1}\left(\iota^{*} \mathbb{L}_{b}\right) \wedge c_{1}\left(R_{c}\right)=\int_{D_{a} \cap \ell_{b}} c_{1}\left(R_{c}\right)=0 \\
& \int_{D_{a}} c_{1}\left(R_{b}\right) \wedge c_{1}\left(R_{c}\right)=\eta_{a b c}
\end{aligned}
$$

branes one can define a holomorphic rank $n_{a}$ bundle (with $n_{a}$ dividing $N_{a}$ ) and embed its structure group $U\left(n_{a}\right)$ into the original $U\left(N_{a}\right)$ theory. This breaks the four-dimensional gauge group down to the commutant $U\left(N_{a} / n_{a}\right)$ of $U\left(n_{a}\right)$ in $U\left(N_{a}\right)$. See [52,53, for a general discussion in terms of D9-branes on Calabi-Yau manifolds.

${ }^{9}$ In the sequel we will sometimes omit the superscripts in $L_{a}^{(i)}$ to avoid cluttering of notation. 
where $\kappa_{a b c}$ and $\eta_{a b c}$ are not necessarily zero. We conclude that the integral over a divisor of a pull-back two form wedge a two-form which is trivial in $Y$ vanishes. As will be detailed below, this implies that a bundle in the cohomology which is trivial in $Y$ but non-trivial on $D_{a}$ does not affect the chiral spectrum, the D-term supersymmetry conditions and the D5-brane tadpole of the brane configuration. However, it does affect the gauge symmetry, the D3-brane tadpole and the non-chiral spectrum of the model.

\section{Quantisation condition}

Essential both for consistency of the theory and for concrete applications is to appreciate the correct quantisation conditions on the gauge flux. Following [42] they are determined by requiring that the worldsheet path integral for an open string wrapping the two-cycle $\Sigma$ with boundary $\partial \Sigma$ along $D_{a}$ be single-valued. Consider first a single brane wrapping the divisor $D_{a}$ and carrying Abelian gauge flux $F_{a}$. The quantity to be well-defined is given by

$$
\operatorname{Pfaff}(D) \exp \left(i \int_{\partial \Sigma} A_{a}\right) \exp \left(i \int_{\Sigma} B\right)
$$

in terms of the Pfaffian of the Dirac operator, the connection $A$ of the Abelian gauge bundle and the $B$-field. If $D_{a}$ is not Spin, that is, if $c_{1}\left(K_{D_{a}}\right) \neq 0 \bmod 2$, the Pfaffian picks up a holonomy upon transporting $\partial \Sigma$ around a loop on $D_{a}$ [42. This holonomy must be cancelled by the second factor in eq. (6) . For internal line bundles this is guaranteed if the gauge flux obeys the condition

$$
\int_{\omega} F_{a}+\frac{1}{2} \int_{\omega} K_{D_{a}} \in \mathbb{Z} \quad \forall \omega \in H_{2}\left(D_{a}, \mathbb{Z}\right),
$$

or equivalently, using our convention eq. (3),

$$
c_{1}\left(L_{a}\right)-\iota^{*} B+\frac{1}{2} c_{1}\left(K_{D_{a}}\right) \in H_{2}\left(D_{a}, \mathbb{Z}\right) .
$$

Note in particular that for trivial $B$ flux along $D_{a}, \iota^{*} B=0$, the Abelian gauge bundle on the single brane $D_{a}$ has to be half-integen 10 quantised if the divisor $D_{a}$ is not Spin.

\footnotetext{
${ }^{10}$ The quantisation condition eq. (8) with non-trivial B-field is related to the concept of vector bundles without vector structure [57] in Type I theory as studied recently, for example, in [58,55].
} 
This condition is readily generalised to line bundles on stacks of D-branes. The probe argument of [42] now implies that the path integral has to be well-defined for every disk worldsheet $\Sigma$ with boundary on each of the branes in the stack of $N_{a}$ D-branes wrapping $D_{a}$. This requires

$$
T_{0}\left(c_{1}\left(L_{a}^{(0)}\right)-\iota^{*} B\right)+\sum_{i} T_{i} c_{1}\left(L_{a}^{(i)}\right)+\frac{1}{2} T_{0} c_{1}\left(K_{D_{a}}\right) \in H_{2}\left(D_{a}, \mathbb{Z}\right)_{N_{a} \times N_{a}} .
$$

where the notation on the right hand side means that all elements of the $N_{a} \times N_{a}$ matrix on the left hand side are in $H_{2}\left(D_{a}, \mathbb{Z}\right)$. One concludes that depending on the precise from of $T_{i}$ the bundles $L_{a}^{(i)}$ can in general be fractionally quantised, a fact that will be very important for our applications.

A second constraint arises for the continuous $B_{-}$moduli in $H_{-}^{2}$ : the restriction to $D_{a}$ of the characteristic class $\zeta$ of the $B_{-}$-field, introduced in [42], has to equal the third Stiefel-Whitney class of $D_{a}$. Recall from [42] that modulo torsion, $\zeta$ is given by the field strength $H=d B_{-}$and that for complex divisors the third Stiefel-Whitney class is always zero. Moreover, for all surfaces considered in this paper, we have $H^{3}\left(D_{a}, \mathbb{Z}\right)=0$ so that $H=d B_{-}$always restricts to zero on the divisor. Therefore no further condition on the B-field moduli $G^{i}$ introduced in (1) arises from these considerations.

\section{Orientifold action}

Let us now describe the orientifold action on the gauge flux. To this end note that the orientifold action $\sigma: D \rightarrow D^{\prime}$ induces a map on cohomology, $\sigma^{*}: H^{2}\left(D_{a}, \mathbb{Z}\right) \rightarrow$ $H^{2}\left(D_{a}^{\prime}, \mathbb{Z}\right)$. The full orientifold action on a vector bundle on $D_{a}$ is given by the composition $\sigma^{*} \Omega_{p}$. Here $\Omega_{p}$ acts as dualisation, $L_{a} \rightarrow L_{a}^{\vee}$. In particular, the Chern character of the image bundle is

$$
\operatorname{ch}_{k}\left(L_{a}^{\prime}\right)=(-1)^{k} \sigma^{*} \operatorname{ch}_{k}\left(L_{a}\right)=\sigma^{*} \operatorname{ch}_{k}\left(L_{a}^{\vee}\right) .
$$

We now discuss the three cases introduced at the beginning of Subsection 2.1 in turn. In the first situation, where not even the homology class of the brane is preserved, one can define two divisors $D_{a}^{ \pm}$and two vector bundles $L_{a}^{ \pm}$by setting

$$
D_{a}^{ \pm}=D_{a} \cup\left( \pm D_{a}^{\prime}\right),\left.\quad L_{a}^{ \pm}\right|_{D_{a}}=L_{a},\left.\quad L_{a}^{ \pm}\right|_{D_{a}^{\prime}}= \pm L_{a}^{\prime},
$$

where $-D_{a}^{\prime}$ is the cycle $D_{a}^{\prime}$ with reversed orientation. Upon setting $H^{2}\left(D_{a}^{+}\right)=$ $H^{2}\left(D_{a}\right) \oplus H^{2}\left(D_{a}^{\prime}\right)$ and decomposing the latter into positive and negative eigenspaces under $\sigma^{*}, H^{2}\left(D_{a}^{+}\right)=H_{+}^{2}\left(D_{a}^{+}\right) \oplus H_{-}^{2}\left(D_{a}^{+}\right)$[55], it follows that $c_{1}\left(L_{a}^{+}\right) \in H_{-}^{2}\left(D_{a}^{+}\right)$. 
In the second case the homology class is preserved but the brane is not point-wise fixed. Hence, the homology class of $D_{a}^{-}$in eq. (11) is trivial and we can use $\left[D_{a}\right]=$ $\frac{1}{2}\left[D_{a}^{+}\right]$. The degree-2 cohomology group of $D_{a}$ thus splits again as $H^{2}\left(D_{a}, \mathbb{Z}\right)=$ $H_{+}^{2}\left(D_{a}\right) \oplus H_{-}^{2}\left(D_{a}\right)$. On the covering space of the orientifold one requires an even number of branes in the homology class of $D_{a}$ which are pairwise identified under the involution $\sigma$. Clearly, this corresponds to an integer number of branes on $D_{a}^{+}$. The Chern class $c_{1}\left(L_{a}\right)$ on $D_{a}$ is in the full $H^{2}\left(D_{a}\right)$ and $\left(D_{a}, L_{a}\right)$ is mapped to $\left(D_{a}, L_{a}^{\prime}\right)$ as in eq. (10).

In the third case, for $D_{a}$ on top of the orientifold, $H^{2}\left(D_{a}\right)=H_{+}^{2}\left(D_{a}\right)$ and $\left(D_{a}, L_{a}\right)$ is mapped to $\left(D_{a}, L_{a}^{\vee}\right)$. This case directly parallels the situation for D9-branes in Type I compactifications. An odd number of branes stuck on top of the orientifold plane is not possible, as discussed recently in [23]. Formally we therefore work upstairs with the system $2 N_{a} D_{a}$ carrying the invariant bundle $L_{a} \oplus L_{a}^{\vee}$.

\subsection{Tadpole Cancellation for Intersecting D7-Branes}

In consistent compactifications it is crucial to cancel the tadpoles of the space-time filling intersecting D7-branes. Satisfying the tadpole cancellation condition ensures that the spectrum is free of non-Abelian gauge anomalies. In general, D7-branes carry also induced D3- and D5- charges arising due to a non-trivial gauge-field configuration on the seven branes and through curvature corrections. All induced tadpoles for a compactification have to be cancelled.

Throughout this article we will be working upstairs on the ambient Calabi-Yau manifold before taking the quotient by $\sigma$. Recall that the K-theoretic charges $\Gamma$ of a D7-brane and the O7-plane along divisors $D_{a}$ and $D_{O 7}$ are encoded in the ChernSimons coupling to the closed RR-forms $2 \pi \int_{\mathcal{R}^{1,3} \times D_{a}} \sum_{2 p} C_{2 p} \Gamma$. Concretely these are given by

$$
\begin{aligned}
& S_{D 7}=2 \pi \int_{\mathcal{R}^{1,3} \times D_{a}} \sum_{2 p} C_{2 p} \operatorname{tr}\left[e^{\frac{1}{2 \pi} \mathcal{F}_{a}}\right] \sqrt{\frac{\hat{A}(T D)}{\hat{A}(N D)}}, \\
& S_{O 7}=-16 \pi \int_{\mathcal{R}^{1,3} \times D_{O 7}} \sum_{2 p} C_{2 p} \sqrt{\frac{L\left(\frac{1}{4} T D_{O 7}\right)}{L\left(\frac{1}{4} N D_{O 7}\right)}}
\end{aligned}
$$

in terms of the $\hat{A}$-roof and the Hirzebruch genus associated with the tangent and normal bundles to the respective divisors. The D7-, D5-, and D3-brane charges follow upon straightforward decomposition of eq. (12). 
Let us start by discussing cancellation of the D7-charge. In a consistent orientifold compactification the D7-brane charge has to equal the charge carried by the O7planes,

$$
\sum_{a} N_{a}\left(\left[D_{a}\right]+\left[D_{a}^{\prime}\right]\right)=8\left[D_{\mathrm{O} 7}\right]
$$

where the sum is over all D7 ${ }_{a}$-branes. Since supersymmetric D7-branes together with their orientifold images wrap cycles $D_{a}+D_{a}^{\prime}$ in the homology classes of $H_{4}^{+}(Y)$, the orientifold invariant charges are captured by the Poincaré dual cohomology $H_{+}^{2}(Y)$ on the ambient Calabi-Yau manifold $Y$.

A net D5-brane charge can be induced by a gauge-field configuration $\mathcal{F}_{a}$ on $D_{a}$ (and the respective orientifold images) if there exist non-trivial elements in $H_{-}^{2}(Y)$. The D5-brane charge along the element $\omega_{b} \in H^{2}(Y, \mathbb{Z})$ of a stack of $N_{a}$ branes carrying Abelian gauge flux as in eq. (3) reads

$$
\Gamma_{\omega}^{D 5}=\frac{1}{2 \pi} \int_{Y} \omega \wedge\left[D_{a}\right] \wedge \operatorname{tr} \mathcal{F}_{a}
$$

where

$$
\frac{1}{2 \pi} \operatorname{tr} \mathcal{F}_{a}=\sum_{I} \operatorname{tr}\left[T_{I}\right] \quad c_{1}\left(L_{a}^{(I)}\right), \quad I=0, i .
$$

The condition for cancellation of D5-brane charge therefore takes the form

$$
\sum_{a} \int_{Y} \omega \wedge\left(\left[D_{a}\right] \wedge \operatorname{tr} \mathcal{F}_{a}+\left[D_{a}^{\prime}\right] \wedge \operatorname{tr} \mathcal{F}_{a^{\prime}}\right)=0
$$

and has to be satisfied for all elements $\omega \in H_{-}^{2}(Y)$. Clearly this condition is vacuous if all branes are of the type $\left[D_{a}\right]=\left[D_{a}^{\prime}\right]$ and $c_{1}\left(L_{a^{\prime}}^{(I)}\right)=c_{1}\left(\left(L_{a}^{(I)}\right)^{\vee}\right)$, but it may be quite restrictive in more general situations.

The general condition for cancellation of the D3-brane tadpole takes the form

$$
\left(N_{\mathrm{D} 3}+N_{\mathrm{D} 3^{\prime}}\right)+N_{\mathrm{flux}}-\sum_{a}\left(Q_{\mathrm{D} 7}^{a}+Q_{\mathrm{D} 7}^{\prime a}\right)=\frac{N_{\mathrm{O} 3}}{2}+Q_{\mathrm{O} 7}
$$

Here $N_{\mathrm{D} 3}$ counts the number of $D 3$-branes, each of which is accompanied by its orientifold image11. $N_{\text {flux }}$ denotes the possible contributions from $G_{3}=F_{3}+\tau H_{3}$

\footnotetext{
${ }^{11}$ In particular, if $n_{D 3}$ D3-branes lie inside an O7-plane they come together with their images and yield gauge group $S p\left(2 n_{D 3}\right)$.
} 
form flux, which is in particular important for complex structure moduli stabilisation. The induced D3-charge on the O7-planes is given by

$$
\begin{aligned}
Q_{\mathrm{O} 7}=\frac{\chi\left(D_{\mathrm{O} 7}\right)}{6} & =\frac{1}{6} \int_{Y} c_{2}\left(D_{\mathrm{O} 7}\right) \wedge\left[D_{\mathrm{O} 7}\right] \\
& =\frac{1}{6} \int_{Y}\left[D_{\mathrm{O} 7}\right]^{3}+c_{2}\left(T_{Y}\right) \wedge\left[D_{\mathrm{O} 7}\right] .
\end{aligned}
$$

If a stack of $N_{a}$ D7-branes wraps a smooth divisor of type 1 or 3, as defined on page 11, their D3-charge reads

$$
Q_{\mathrm{D} 7}^{a}=N_{a} \frac{\chi\left(D_{a}\right)}{24}+\frac{1}{8 \pi^{2}} \int_{D_{a}} \operatorname{tr} \mathcal{F}_{a}^{2}
$$

with

$$
\frac{1}{8 \pi^{2}} \operatorname{tr} \mathcal{F}_{a}^{2}=\frac{1}{2} \sum_{I, J} \operatorname{tr}\left[T_{I} T_{J}\right] c_{1}\left(L_{a}^{(I)}\right) c_{1}\left(L_{a}^{(J)}\right)
$$

More subtle is the case 2, since eq. (17) will be modified as discussed in [21,23]. One replaces the Euler characteristic by

$$
Q_{\mathrm{D} 7}^{a}=N_{a} \frac{\chi_{o}\left(D_{a}\right)}{24}+\frac{1}{8 \pi^{2}} \int_{D_{a}} \operatorname{tr} \mathcal{F}_{a}^{2}, \quad \chi_{o}\left(D_{a}\right)=\chi\left(\Sigma_{a}\right)-n_{\mathrm{pp}},
$$

where $\Sigma_{a}$ is an auxiliary surfaces $\Sigma_{a}$ obtained by blowing up the singular points in $D_{a}$, while $n_{\mathrm{pp}}$ counts the number of pinch points in $D_{a}$.

The relation to the F-theory D3-brane tadpole condition becomes obvious if one slightly reorders the terms in eq. (17) and divides by two,

$$
N_{\mathrm{D} 3}+\frac{N_{\text {flux }}}{2}+N_{\text {gauge }}=\frac{N_{\mathrm{O} 3}}{4}+\frac{\chi\left(D_{\mathrm{O} 7}\right)}{12}+\sum_{a} N_{a} \frac{\chi_{o}\left(D_{a}\right)}{24}
$$

with

$$
N_{\text {gauge }}=-\sum_{a} \frac{1}{8 \pi^{2}} \int_{D_{a}} \operatorname{tr} \mathcal{F}_{a}^{2}=-\frac{1}{2} \sum_{a} N_{a} \int_{D_{a}} \sum_{I, J} \operatorname{tr}\left[T_{I} T_{J}\right] c_{1}\left(L_{a}^{(I)}\right) c_{1}\left(L_{a}^{(J)}\right) .
$$

The right-hand side of equation (22) is precisely $\chi\left(Y_{4}\right) / 24$ in the F-theory lift of this Type IIB orientifold, where $Y_{4}$ denotes the Calabi-Yau fourfold. This implies that generically each topologically different arrangement of D7-branes cancelling the RR 
eight-form tadpole constraints lifts to a different Calabi-Yau fourfold with different Euler characteristic. For the trivial solution with eight D7-branes placed right on top of the smooth orientifold plane with $n_{\mathrm{pp}}=0$ the right hand side of (22) simplifies to $\frac{N_{\mathrm{O} 3}}{4}+\frac{\chi\left(D_{\mathrm{O} 7}\right)}{4}$. It is a consistency check that this number is indeed an integer.

Let us emphasise that for the cancellation of anomalies only the D7 and D5tadpole constraints are important. The D3-brane tadpole is in some sense only related to the non-chiral sector of the D-brane theory. This is related to the fact that a D3-brane can never carry any chiral modes, as it can in principle be moved to a position away from the D7-branes. The expectation is that a globally consistent Type IIB orientifold model with a supersymmetric D7- and D5-brane sector lifts up to F-theory on a compact Calabi-Yau fourfold. The cancellation of the D3-brane tadpole is an additional attribute both in Type IIB orientfolds and in F-theory models. Taking also into account that for moduli stabilisation and the realisation of inflation, the presence of (a small number of) anti-D3-branes is very welcome, in this paper we take all the D7- and D5-brane supersymmetry constraints very serious but are a bit more relaxed about the existence of anti- D3-branes in the system. In fact, we will find that in our semi-realistic GUT examples the D3-brane tadpole can easily be saturated by already modest addition of gauge fluxes on the D7-branes.

\section{Role of $\mathrm{B}_{-}-$moduli}

Before proceeding we would like to comment on the role of the continuous $B_{-}$-moduli appearing in $\mathcal{F}$ and thus in the D-brane charges. It is natural to wonder how to reconcile their contribution with the discrete nature of a quantity such as the D5- or D3-brane charge.

In fact the $B_{-}$moduli decouple from the tadpole equations by means of the $D 7$ brane tadpole cancellation condition (13) and the simple observation that the $B_{-}$ field restricts trivially to the O7-plane,

$$
\int_{D_{O 7}} \omega \wedge B_{-}=0, \quad \forall \omega \in H_{-}^{2}(Y) .
$$

Concretely, the $B_{-}$-contribution to the D5-brane tadpole condition (16)

$$
\sum_{a} N_{a} \int_{Y} \omega \wedge\left(\left[D_{a}\right] \wedge B_{-}+\left[D_{a}^{\prime}\right] \wedge B_{-}\right)=8 \int_{Y} \omega \wedge\left[D_{O 7}\right] \wedge B_{-}=0
$$

indeed vanishes due to (13) and (24). 
To isolate the $B_{-}$-moduli in the D3-brane tadpole let us introduce the quantity

$$
c_{1}\left(\widetilde{L}_{a}\right)=c_{1}\left(L_{a}\right)-B_{-}
$$

and rewrite the induced D3-brane tadpole as

$$
-\sum_{a} N_{a}\left[\int_{D_{a}}\left(c_{1}\left(\widetilde{L}_{a}\right)+B_{-}\right)^{2}+\int_{D_{a}^{\prime}}\left(c_{1}\left(\widetilde{L}_{a}^{\prime}\right)+B_{-}\right)^{2}\right] .
$$

For simplicity we are sticking to gauge flux $\frac{1}{2 \pi} \mathcal{F}=T_{0} c_{1}\left(L_{a}\right)$. For the mixed term we find that

$$
-\sum_{a} N_{a} \int_{Y}\left(\left[D_{a}\right] \wedge c_{1}\left(\widetilde{L}_{a}\right)+\left[D_{a}^{\prime}\right] \wedge c_{1}\left(\widetilde{L}_{a}^{\prime}\right)\right) \wedge B_{-}=0,
$$

where we have used the D5-brane tadpole cancellation condition (16). Finally, we evaluate

$$
-\sum_{a} N_{a} \int_{Y}\left(\left[D_{a}\right] \wedge B_{-}^{2}+\left[D_{a}^{\prime}\right] \wedge B_{-}^{2}\right)=-8 \int_{D_{O 7}} B_{-}^{2}=0
$$

so that as anticipated the continuous $B_{-}$-moduli do not appear in the tadpole cancellation conditions.

\section{K-Theory charge cancellation}

Apart from cancellation of these homological charges, also all K-theoretic torsion charges have to sum up to zero. In general it is a very non-trivial task to compute all in particular torsional K-theory constraints. However, according to the probe brane argument of [60] cancellation of torsion charges is equivalent to absence of a global $S U(2)$ Witten anomaly on the worldvolume of every probe brane supporting symplectic Chan-Paton factors. In concrete compactifications this amounts to requiring an even number of fundamental representations in the sector between the physical D7-branes and each symplectic probe brane. Note that in a concrete model determining all symplectic four-cycles is also far from trivial.

\subsection{The Massless Spectrum}

For applications to phenomenology it is essential to understand the massless matter arising from open strings stretching between two stacks of D7-branes.

Non-chiral matter transforming in the adjoint representation emerges from strings with both endpoints on the same D-brane along $D_{a}$. It consists of the vector multiplet 
together with $h^{1,0}\left(D_{a}\right)$ and $h^{2,0}\left(D_{a}\right)$ chiral multiplets describing the Wilson line and deformation moduli of the D7-branes. Matter in the bifundamental representation 12 $\left(\bar{N}_{a}, N_{b}\right)$ and $\left(N_{a}, N_{b}\right)$ arises from open strings stretching between two different D7branes in the $(a, b)$ and $\left(a^{\prime}, b\right)$ sector, respectively. Intersections between a brane and its image, that is, of type $\left(a^{\prime}, a\right)$, yield matter in the (anti)symmetric representation. For example, if all branes are on top of a $O 7^{(-)}$-plane, then all states in the $\left(a^{\prime}, a\right)$ sector are anti-symmetrised. On an invariant brane with four Dirichlet-Neumann boundary conditions with an $O 7^{(-)}$-plane, the $\left(a^{\prime}, a\right)$ states are symmetrised. The chiral spectrum is summarised in Table 1, see also [61]. For the concrete computation

\begin{tabular}{|c|c|c||c|}
\hline \hline sector & $U\left(N_{a}\right)$ & $U\left(N_{b}\right)$ & chirality \\
\hline \hline$(a b)$ & $\square_{(-1)}$ & $\square_{(1)}$ & $I_{a b}$ \\
\hline$\left(a^{\prime} b\right)$ & $\square_{(1)}$ & $\square_{(1)}$ & $I_{a^{\prime} b}$ \\
\hline$\left(a^{\prime} a\right)$ & $\square_{(2)}$ & 1 & $\frac{1}{2}\left(I_{a^{\prime} a}+2 I_{\mathrm{O} 7 \mathrm{a}}\right)$ \\
\hline$\left(a^{\prime} a\right)$ & $\square(2)$ & 1 & $\frac{1}{2}\left(I_{a^{\prime} a}-2 I_{\mathrm{O} 7 \mathrm{a}}\right)$ \\
\hline \hline
\end{tabular}

Table 1: Chiral spectrum for intersecting D\%-branes. The subscripts denote $U(1)$ charges.

of the chiral index $I_{a b}$ and to determine the vector-like spectrum we have to distinguish two situations according to the localisation of matter on sub-loci of different dimensions. For simplicity we again only discuss the case where all D7-branes carry holomorphic line bundles.

\subsubsection{Matter Divisors}

For two D7-branes wrapping the same divisor $D_{a}=D_{b}=D$ and carrying line bundles $L_{a}$ and $L_{b}$, matter in the bifundamental representation $\left(\bar{N}_{a}, N_{b}\right)$ is counted by the extension groups 62 ]

$$
\operatorname{Ext}^{n}\left(\iota_{*} L_{a}, \iota_{*} L_{b}\right), \quad n=0, \ldots 3
$$

\footnotetext{
${ }^{12}$ For the general overview we only consider diagonal embeddings and postpone a discussion of more general line bundles to the applications in Section 5 .
} 
where $i: D \rightarrow Y$ defines the embedding of $D$ in the Calabi-Yau $Y$. The value $n=1$ refers to anti-chiral multiplets transforming as $\left(\bar{N}_{a}, N_{b}\right)$, while $n=2$ corresponds to chiral multiplets in the same representation. For consistency, the states counted by the groups corresponding to $n=0$ and $n=3$ have to absent. These states do not correspond to matter fields but rather gauge fields and have been named ghosts in [13]. We show in Subsection 2.4 that for supersymmetric configurations with the Kähler form inside the Kähler cone these ghosts are automatically absent. By running through the spectral sequence, one can show that the sheaf extension groups eq. (30) are given by appropriate cohomology groups for line bundles on the divisor $D$, concretely

$$
\begin{array}{rr}
\operatorname{Ext}^{0}\left(\iota_{*} L_{a}, \iota_{*} L_{b}\right)=H^{0}\left(D, L_{a} \otimes L_{b}^{\vee}\right), \\
\operatorname{Ext}^{1}\left(\iota_{*} L_{a}, \iota_{*} L_{b}\right)=H^{1}\left(D, L_{a} \otimes L_{b}^{\vee}\right)+H^{0}\left(D, L_{a} \otimes L_{b}^{\vee} \otimes N_{D}\right), \\
\operatorname{Ext}^{2}\left(\iota_{*} L_{a}, \iota_{*} L_{b}\right)=H^{2}\left(D, L_{a} \otimes L_{b}^{\vee}\right)+H^{1}\left(D, L_{a} \otimes L_{b}^{\vee} \otimes N_{D}\right), \\
\operatorname{Ext}^{3}\left(\iota_{*} L_{a}, \iota_{*} L_{b}\right)= & H^{2}\left(D, L_{a} \otimes L_{b}^{\vee} \otimes N_{D}\right) .
\end{array}
$$

By Serre duality and $N_{D}=K_{D}$ we can relate $H^{i}\left(D, L_{a} \otimes L_{b}^{\vee} \otimes N_{D}\right)=H^{2-i}\left(D, L_{a}^{\vee} \otimes\right.$ $\left.L_{b}\right)$. It straightforwardly follows that for the chiral index $I_{a b}$ counting bifundamental matter transforming as $\left(\bar{N}_{a}, N_{b}\right)$ one obtains

$$
\begin{aligned}
I_{a b}^{\text {bulk }} & =\sum_{n=0}^{3}(-1)^{n} \operatorname{dim} \operatorname{Ext}^{n}\left(\iota_{*} L_{a}, \iota_{*} L_{b}\right) \\
& =\chi\left(D, L_{a} \otimes L_{b}^{\vee}\right)-\chi\left(D, L_{a} \otimes L_{b}^{\vee} \otimes N_{D}\right) \\
& =-\int_{Y}[D] \wedge[D] \wedge\left(c_{1}\left(L_{a}\right)-c_{1}\left(L_{b}\right)\right) .
\end{aligned}
$$

In these conventions $I_{a b}>0$ if there is an excess of chiral states in the representation $\left(\bar{N}_{a}, N_{b}\right)$. Note that this expression only depends on the components of $c_{1}\left(L_{i}\right)$ which are non-trivial on the ambient Calabi-Yau manifold, cf. eq. (55).

We have the additional freedom to twist the line bundle $L_{a}$ on $D$ by a line bundle $R_{a}$ with $\iota_{*} R_{a}=\mathcal{O}$. This does not change the chiral spectrum, though it can change the vector-like one and will in general contribute to the D3-tadpole.

\subsubsection{Matter Curves}

If the two D7-branes wrap different divisors $D_{a}$ and $D_{b}$ intersecting over a curve $C$ of genus $g$ and carrying line bundles $L_{a}$ and $L_{b}$, the cohomology groups counting 
matter in $\left(\bar{N}_{a}, N_{b}\right)$ are

$$
H^{i}\left(C, L_{a}^{\vee} \otimes L_{b} \otimes K_{C}^{\frac{1}{2}}\right) .
$$

Here $i=0$ and $i=1$ refer to chiral and anti-chiral states in the representation $\left(\bar{N}_{a}, N_{b}\right)$, respectively 13 . The chiral index $I_{a b}$ counting the excess of chiral over antichiral states transforming as $\left(\bar{N}_{a}, N_{b}\right)$ follows from the Riemann-Roch-Hirzebruch theorem as

$$
I_{a b}^{\text {loc. }}=\chi\left(C, L_{a}^{\vee} \otimes L_{b} \otimes K_{C}^{\frac{1}{2}}\right)=-\int_{Y}\left[D_{a}\right] \wedge\left[D_{b}\right] \wedge\left(c_{1}\left(L_{a}\right)-c_{1}\left(L_{b}\right)\right) .
$$

In terms of extension groups we therefore get

$$
\operatorname{Ext}^{i}\left(\iota_{*} L_{a}, \iota_{*} L_{b}\right)= \begin{cases}0 & i=0 \\ H^{1}\left(C, L_{a}^{\vee} \otimes L_{b} \otimes K_{C}^{\frac{1}{2}}\right) & i=1 \\ H^{0}\left(C, L_{a}^{\vee} \otimes L_{b} \otimes K_{C}^{\frac{1}{2}}\right) & i=2 \\ 0 & i=3 .\end{cases}
$$

Finally, the index $I_{O 7 a}$ in Table 1 is

$$
I_{O 7 a}=\int_{Y}\left[D_{O 7}\right] \wedge\left[D_{a}\right] \wedge c_{1}\left(L_{a}\right),
$$

reflecting the absence of a gauge bundle on top of the orientifold plane.

We leave it to the readers to convince themselves that, as in the context of the tadpole cancellation conditions, the $B_{-}$-moduli also drop out automatically from all cohomology groups describing the massless spectrum.

\subsection{F- and D-Term Supersymmetry Constraints}

Let us discuss the constraints which need to be imposed in order for the brane configuration to be supersymmetric. In the four-dimensional effective action these constraints manifest themselves through the vanishing of $\mathrm{F}$ - and D-terms in the vacuum. In the following we will discuss both sets of constraints in turn.

We first turn to the supersymmetry constraints imposed by the vanishing of the D-terms. Recall that the D-term induced by a gauging of a field-independent

\footnotetext{
${ }^{13}$ Note the different assignment of chiral and anti-chiral multiplets with the extension groups of even and odd degree for bulk and localised matter. This can be derived, for example, by T-duality from the analogous phenomenon in D9-D5 systems [52,53].
} 
symmetry with Killing vector $X_{a}^{L}$ is of the form $D_{a}=\bar{X}_{a}^{L} \partial_{\bar{M}^{L}} K$, where $K$ is the Kähler potential. Let us recall the induced gauging for the complex scalars $T_{I}$ and $G^{i}$ defined in (11). A gauging of $T_{I}$ can be induced for a non-trivial line bundle on a D7brane $\left(D_{a}, L_{a}\right)$, while $G^{i}$ can be gauged if there exists a $D_{a}^{\prime}$ which is not homologous to $D_{a}$, that is, if we are in the case 1 defined at the beginning of Subsection 2.1, page 11. The Killing vectors for these gaugings are of the form

$$
X_{a I}=\int_{D_{a}}\left[\Gamma_{I}^{+}\right] \wedge c_{1}\left(\widetilde{L}_{a}\right), \quad X_{a}^{i}=\int_{D_{a}}\left[\gamma_{-}^{i}\right] \wedge B_{-},
$$

where $\Gamma_{I}^{+}, \gamma_{-}^{i}$ are the four- and two-cycles introduced in eq. (11) to define $T_{I}, G^{i}$. Note that there is no continuous moduli dependence in $X_{a I}$ since we have explicitly split off the $B_{-}$field as in eq. (26) . One next notes that [49, 63]

$$
\partial_{T_{I}} K \sim r^{I}, \quad \partial_{G^{i}} K \sim s_{i}
$$

where $r^{I}, s_{i}$ arise in the expansions $J=r^{I}\left[\Gamma_{I}^{+}\right]$and $J \wedge B_{-}=s_{i}\left[\gamma_{-}^{i}\right]$. It is important to note that the expression for $\partial_{T_{I}} K$ in eq. (38) is also valid away from the large volume limit. For example, one of the $r^{I}$ can become small while $\partial_{G^{i}} K$ will receive additional corrections, for example, due to world-sheet instantons. We thus encounter a moduli dependent Fayet-Iliopoulos term for the configuration of the form [51, 55]

$$
\xi_{a} \sim \int_{D_{a}} \iota^{*} J \wedge\left(c_{1}\left(\widetilde{L}_{a}\right)+B_{-}\right)=\int_{D_{a}} \iota^{*} J \wedge c_{1}\left(L_{a}\right) .
$$

Note that $\xi_{a}$ depends on the pullback of the Kähler form $J$ of $Y$ to the D7-brane and as a consequence of eq. (5) only on the components of $c_{1}\left(L_{a}\right)$ which are non-trivial on $Y$. Furthermore the $B_{-}$-moduli, encoded in $c_{1}\left(L_{a}\right)$, do not drop out of the D-terms. For vanishing VEVs of the chiral fields charged under the $U(1)$ supported on the D7branes, the D-term supersymmetry condition requires these FI-terms to vanish. This imposes conditions on the combined Kähler and $B_{-}$moduli space. As long as the Kähler moduli are chosen such that $J$ is indeed invariant under the orientifold action, the Fayet-Iliopoulos term for $\left(D_{a}, L_{a}\right)$ and $\left(D_{a}^{\prime}, L_{a}^{\prime}\right)$ coincide. We will encounter that in a concrete example in Subsection 6.2.

For line bundles $L_{a} \neq \mathcal{O}$ satisfying the D-term constraint eq. (39), we will now derive two important consequences:

\section{No ghosts}

First, we realise that the FI-term is nothing else than the slope $\mu\left(L_{a}\right)$ of the line bundle $L_{a}$. Now to come back to the question of ghosts in the massless spectrum in 
eq. (31), it is important to recall the general fact that

- For two vector bundles $V_{a}, V_{b}$ of equal slope and rank, $\mu\left(V_{a}\right)=\mu\left(V_{b}\right)$ and $\operatorname{rk}\left(V_{a}\right)=\operatorname{rk}\left(V_{b}\right)$, the existence of a map $0 \rightarrow V_{a} \rightarrow V_{b}$ implies that $V_{a}=V_{b}$.

We thus conclude for the extension groups between two supersymmetric line bundles $L_{a} \neq L_{b}$ that $\operatorname{Ext}^{0}\left(\iota_{*} L_{a}, \iota_{*} L_{b}\right)=\operatorname{Ext}^{3}\left(\iota_{*} L_{a}, \iota_{*} L_{b}\right)=0$. Indeed, if $H^{0}\left(D, L_{a} \otimes L_{b}^{\vee}\right)$ were non-vanishing, we could define a map $0 \rightarrow \mathcal{O} \rightarrow L_{a} \otimes L_{b}^{\vee}$ where both $\mathcal{O}$ and, by hypothesis, $L_{a} \otimes L_{b}^{\vee}$ have vanishing slope. Therefore, $L_{a}=L_{b}$ in contradiction to our assumption. The same reasoning for the dual bundle $L_{a}^{\vee} \otimes L_{b}$ shows that $\operatorname{Ext}^{3}\left(\iota_{*} L_{a}, \iota_{*} L_{b}\right)=0$.

\section{D3-tadpole contribution}

Second we note that for line bundles with vanishing slope for a Kähler form inside the Kähler cone, the contribution of the gauge flux to the D3-brane tadpole always has the same sign

$$
-\frac{N_{a}}{2} \int_{D_{a}} c_{1}^{2}\left(L_{a}\right) \geq 0
$$

Indeed, on a surface $D_{a}$ the set of $c_{1}\left(L_{a}\right)$ with vanishing slope is given by $H_{2}\left(D_{a}\right)-$ $\{M \cup-M\}$, where $M$ denotes the Mori cone of $D_{a}$. However, the Mori cone contains all classes $C$ with $C^{2}>0$ and $C \cdot K>0$. Therefore, $c_{1}^{2}\left(L_{a}\right) \leq 0$. This result implies that for supersymmetric brane configurations the possible choices of line bundles are rather limited if we do not want to introduce anti-D3-branes in the system.

Finally, let us mention that the other supersymmetry conditions, namely the holomorphy of the divisor and the bundle, arise from a superpotential

$$
W_{D 7}=\int_{\mathcal{C}\left(L_{a}, L_{a}^{\prime}\right)} \Omega,
$$

where $\mathcal{C}\left(L_{a}, L_{a}^{\prime}\right)$ is a chain ending on the two-cycle Poincaré dual to $c_{1}\left(L_{a}^{+}\right)$on the divisor $D_{a}+D_{a}^{\prime}$.

\section{$3 \quad \mathrm{SU}(5)$ GUTs and Their Breaking}

After this discussion of the general model building rules for Type IIB orientifolds with O7- and O3- planes we can now become more specific about the realisation of 
SU(5) Georgi-Glashow GUTs. Parts of the logic are very similar to the implementation of GUT models in Type IIA intersecting D-branes [43, 11,45] as described, for example, in [11,28]. Let us first transfer these rules to our Type IIB setting. Then we move forward to describe how the mechanism of GUT symmetry breaking via $U(1)_{Y}$ flux, exploited by [16] in the local F-theory context, can also be realised in this perturbative orientifold limit.

\subsection{Georgi-Glashow SU(5) GUT}

The starting point is the construction of a $U(5) \times U(1)$ gauge theory from a stack of five D7-branes wrapping a four-cycle $D_{a}$ and one additional brane wrapping $D_{b}$. These brane stacks carry holomorphic bundles $L_{a}$ and $L_{b}$, respectively.

The orientifold action maps $\left(D_{a}, L_{a}\right) \rightarrow\left(D_{a^{\prime}}, L_{a}^{\prime}\right)$ (and similarly for $\left(D_{b}, L_{b}\right)$ ). As previously discussed, this includes the case that $D_{a}$ is invariant under $\sigma$. First we diagonally embed two line bundles $L_{a}$ and $L_{b}$ by identifying their structure group with the diagonal $U(1)_{a}$ and $U(1)_{b}$, respectively. Each of the two Abelian factors $U(1)_{a}$ and $U(1)_{b}$ separately acquires a mass by the Stückelberg mechanism as long as $D_{a}$ and $D_{b}$ are non-trivial homology cycles [54].

A more group theoretic way of describing the gauge group and its matter content is to start with an $S O(12)$ gauge group. The embedding of two line bundles with structure groups $U(1)_{a, b}$ can break this to $U(5) \times U(1)$, where the generators of the two $U(1)$ s are embedded into $S O(12)$ as

$$
\begin{aligned}
U(1)_{a} & \in \operatorname{diag}\left(1_{5 \times 5}, 0 \mid 0,-1_{5 \times 5}\right), \\
U(1)_{b} & \in \operatorname{diag}\left(0_{5 \times 5}, 1 \mid-1,0_{5 \times 5}\right) .
\end{aligned}
$$

The adjoint of $S O(12)$ decomposes into $S U(5) \times U(1)_{a} \times U(1)_{b}$ representations as

$$
\begin{aligned}
{[66]=} & {[24]_{(0,0)}+[1]_{(0,0)}+[10]_{(2,0)}+[\overline{10}]_{(-2,0)}+[5]_{(1,-1)}+[\overline{5}]_{(-1,1)} } \\
& +[5]_{(1,1)}+[\overline{5}]_{(-1,-1)} .
\end{aligned}
$$

To ensure absence of a massless combination of $U(1)$ factors we require that $\left[D_{a}\right]$ and $\left[D_{b}\right]$ be linearly independent in $H_{4}(Y, \mathbb{Z})$. Note that in the presence of further tadpole cancelling D7-branes it has to be ensured that the full mass matrix is of maximal rank. 


\section{The MSSM spectrum}

The massless states transforming in the adjoint representation are given by the deformations of the four-cycles, which are counted by $H^{1}(D, \mathcal{O})$ (Wilson lines) and $H^{2}(D, \mathcal{O})$ (transversal deformations). In principle we could allow for precisely one such adjoint of $S U(5)$, which might break the $S U(5)$ symmetry to the Standard model by the Higgs mechanism. An example of such a surface with $h^{(2,0)}=1$ is $K 3$. However, a complete GUT model relying on this mechanism would have to address the generation of a suitable potential for the GUT Higgs field from string dynamics such that $S U(5)$ is broken dynamically to the Standard Model. Since we will rather be breaking the GUT symmetry by embedding $U(1)_{Y}$ flux, we insist that the $S U(5)$ stack wraps a rigid four-cycle. This is satisfied for del Pezzo surfaces, which have $h^{1,0}(D)=h^{2,0}(D)=0$. In view of the rules of Table 1 the charged GUT spectrum requires the chiral intersection pattern listed in Table 2 .

\begin{tabular}{|c|c||c|c|c|}
\hline \hline state & number & sector & $U(5)$ & $U(1)$ \\
\hline \hline $\mathbf{1 0}$ & 3 & $\left(a^{\prime} a\right)$ & $\Xi_{(2)}$ & 1 \\
$\overline{\mathbf{5}}$ & 3 & $\left(a b^{\prime}\right)$ & $\bar{\square}_{(-1)}$ & $\square_{(-1)}$ \\
\hline $\mathbf{1}_{N}$ & 3 & $\left(b^{\prime} b\right)$ & 1 & $\square_{(2)}$ \\
$\mathbf{5}_{H}+\overline{\mathbf{5}}_{H}$ & $1+1$ & $(a b)$ & $\bar{\square}_{(-1)}$ & $\square_{(1)}$ \\
\hline \hline
\end{tabular}

Table 2: Chiral spectrum for intersecting D\%-brane model. The indices denote the $U(1)$ charges. The last line gives the Higgs particles.

The first two lines contain the antisymmetric representation $\mathbf{1 0}$ of $S U(5)$ and the fundamental $\overline{\mathbf{5}}$. The states from the $b^{\prime} b$ sector are necessary to satisfy the "formal" $U\left(N_{b}\right)$ anomaly $(3 \times(4+1)-3 \times 5=0)$ and carry the charges of right-handed neutrinos. States from the $\left(a^{\prime} b\right)$ carry the right quantum numbers to be identified with the pair of Higgs fields $\mathbf{5}_{\mathbf{H}}+\overline{\mathbf{5}}_{\mathbf{H}}$. However, one can also realise the Higgs fields from intersections $(a c)$ between the $S U(5)$ brane stack and a third one. In contrast to $S O(10)$ GUTs, here all massless fields are perturbatively realised by open string stretched between stacks of D7-branes.

The various fields are localised on the intersection of the various divisors. As mentioned already in Subsection 2.1, these are either curves or divisors. In the latter 
case one has to compute cohomology classes over a del Pezzo surface, which in general gives also vector-like matter. On the contrary, if two divisors intersect over a curve vector-like states are much easier to suppress. We will exemplify this feature in the concrete examples to be discussed later.

\section{Yukawa couplings}

The Yukawa couplings which give masses to the MSSM fields after GUT and electroweak symmetry breaking are

$$
\mathbf{1 0}^{(2,0)} \mathbf{1 0}^{(2,0)} \mathbf{5}_{\mathbf{H}}^{(1,-1)}, \quad \mathbf{1 0}^{(2,0)} \overline{\mathbf{5}}^{(-1,-1)} \overline{\mathbf{5}}_{\mathbf{H}}{ }^{(-1,1)} \quad \mathbf{1}_{N}^{(0,2)} \overline{\mathbf{5}}^{(-1,-1)} \mathbf{5}_{\mathbf{H}}^{(1,-1)},
$$

where the upper indices denote the Abelian $U(1)_{a} \times U(1)_{b}$ charges. If as indicated we realise the matter and Higgs fields as in Table 2, the last two Yukawa couplings, that is, the ones generating masses for the d-quarks and leptons, are allowed already at the perturbative level. For them to be present the wave functions of the massless modes have to overlap. If all states are localised on curves, this means that the three divisors have to meet at a point. On a Calabi-Yau threefold, this is generically the case. If on the other hand the $\mathbf{1 0}$ and the $\mathbf{1}_{\mathbf{N}}$ arise from the bulk of the GUT brane and the $U(1)_{b}$ brane while the $\overline{\mathbf{5}}$ and the Higgs are localised on curves, the perturbative Yukawa couplings involve the triple-product of the restriction of corresponding powers of $L_{a}$ and $L_{b}$ to the matter curve.

By contrast, it is obvious from their $U(1)$ charges in eq. (44) that the u-quark Yukawa couplings $\mathbf{1 0} \mathbf{1 0} 5_{\mathbf{H}}$ are perturbatively forbidden. For quite some time this was considered the main obstacle to the construction of open string SU(5) GUT models. This no-go was bypassed in [28] where it was pointed out that an isolated, rigid Euclidean D3-brane instanton wrapping a four-cycle $D_{\text {inst }}$ of $O(1)$ type (that is, in particular invariant under orientifold action) can generate these missing Yukawa couplings. This requires extra fermionic charged matter zero modes localised at the intersection of the instanton with the two stacks of D7-branes. Concretely, a necessary condition is that the chiral intersection numbers are

$$
I_{a, \text { inst }}=1, \quad I_{b, \text { inst }}=-1
$$

The resulting six chiral zero modes $\lambda_{a}^{i}, \lambda_{b}, i=1, \ldots 5$, can then be absorbed by the disc diagrams

$$
\mathbf{1 0}^{(2,0)} \lambda_{a}^{i} \lambda_{a}^{j}, \quad \mathbf{1 0}^{(2,0)} \lambda_{a}^{k} \lambda_{a}^{l}, \quad \mathbf{5}_{H}^{(1,-1)} \lambda_{a}^{m} \lambda_{b}
$$


As detailed [28] this results in non-perturbative couplings proportional to

$$
Y^{\alpha} Y^{\beta} \epsilon_{i j k l m} 10_{\alpha}^{i j} 10_{\beta}^{k l} 5_{H}^{m}
$$

with $i, j, \ldots$ denoting $S U(5)$ group indices and $\alpha, \beta$ labelling families. Note that the coupling eq. (47) is of unit rank in family space so that a single instanton gives rise to masses only for one particular generation of u-quarks. This is a consequence of the fact that the multiplicities of the $\lambda^{i}$-modes are only due to their $S U(5)$ Chan-Paton factors.

Of course the amplitude is suppressed by the instanton action

$$
\exp \left(-\frac{1}{2 g_{s}} \int_{D_{\text {inst }}} J \wedge J\right) .
$$

As such the scale of the coupling is independent of the GUT coupling, which is controlled by the cycle volume of the GUT brane. The instanton cycle entering the above suppression, however, is a priori unrelated to the GUT cycle. Still in the perturbative regime $g_{s} \ll 1$ there is the danger that the coupling tends to be too small. In our approach we will eventually take the small $T_{\text {inst }}$ limit of the orientifold model and, besides imposing the tadpole constraints, will require that at least for the third family this Yukawa coupling is generated by a Euclidean D3-brane instanton. Of course we have to ensure that when $T_{\text {inst }} \rightarrow 0$ not the whole manifold degenerates. In principle the large hierarchy in the u-quark masses between the third and the first two families can be engineered by different instantons with suitable suppression.

Very similarly, if the Higgs fields originate from the intersection of the $S U(5)$ branes with a third stack then the bottom Yukawa couplings carry $U(1)^{3}$ charges

$$
\mathbf{1 0}^{(2,0,0)} \overline{\mathbf{5}}^{(-1,-1,0)} \overline{\mathbf{5}}_{\mathbf{H}}{ }^{(-1,0,1)}
$$

and are therefore not gauge invariant any more. In this case, also these couplings can only be generated non-perturbatively by an appropriate D3-brane instanton, which intersects the $U(1)$ stacks $b$ and $c$ just once.

\section{$\operatorname{Dim}=4$ proton decay}

In GUT theories there is the danger of generating dimension-four operators violating baryon or lepton number

$$
U D D, \quad Q D L, \quad L L E
$$


Clearly if present they generate unacceptably fast proton decay. In Georgi-Glashow $S U(5)$ all these operators descend from the

$$
\mathbf{1 0}^{(2,0)} \overline{\mathbf{5}}^{(-1,-1)} \overline{\mathbf{5}}^{(-1,-1)}
$$

coupling, which is perturbatively forbidden due to $U(1)_{b}$ violation. However, as just described, even perturbatively absent couplings can be generated non-perturbatively by D3-brane instantons. In certain domains of the Kaähler moduli space such instanton-induced dimension-four operators would be dangerous. For an instanton to generate such a coupling three situations are in principle conceivable in view of the $U(1)_{a}$ and $U(1)_{b}$ charges: it either carries the six charged matter zero modes $\lambda_{a}^{i}, \bar{\lambda}_{a}^{j}$, $\bar{\lambda}_{b}^{k}$ with $i, j, k=1,2$ or alternatively the four zero modes $\lambda_{a}, \bar{\lambda}_{a}, \bar{\lambda}_{b}^{k}$ with $k=1,2$. The third possibility is that it carries just the two zero modes $\bar{\lambda}_{b}^{k}$ with $k=1,2$. On the other hand, charged matter zero modes from intersections of the instanton with the $S U(5)$ stack always appear in multiples of five. We thus conclude that in absence of any known mechanism to absorb the extra zero modes without pulling down more charged matter fields, in the two first cases no such dangerous dimension-four operators are generated. However, the third option is not a priori excluded. Of course, if such an instanton exists the coupling is exponentially suppressed, but we just learnt in the context of the $\mathbf{1 0 1 0} \mathbf{5}_{\mathbf{H}}$ Yukawa coupling that this need not be the case in the F-theory limit. Therefore, to be on the safe side we require that such an instanton does not exist.

\section{Neutrino masses}

We have already seen that the Yukawa coupling $\mathbf{1}_{\mathbf{N}}{ }^{(0,2)} \overline{\mathbf{5}}^{(-1,-1)} \mathbf{5}_{\mathbf{H}}{ }^{(1,-1)}$ generates Dirac type masses for the neutrinos. In order to realise for instance the see-saw mechanism one also needs Majorana type masses of the order $10^{12}-10^{15} \mathrm{GeV}$. These can be either generated by higher dimensional couplings involving some additional $S U(5)$ singlet fields or by D3-brane instantons [25, 26, 64, 65]. Higher dimensional couplings are of course suppressed by the string scale, so that one needs to explain the high scale of these terms.

For directly generating a mass term

$$
S_{\text {mass }}=M_{N} \mathbf{1}_{N}^{(0,2)} \mathbf{1}_{N}^{(0,2)}
$$

via an instanton, it has to carry the four charged matter zero modes $\lambda_{b}^{i}, i=1, \ldots, 4$. In this case the Majorana mass scale is $M_{s} \exp \left(-S_{\text {inst }}\right)$ which, that is, depending on the size of the four-cycle, can still give a suppression by a few orders of magnitude. 


\subsection{Breaking $\mathrm{SU}(5)$ to $\mathrm{SU}(3) \times \mathrm{SU}(2) \times \mathrm{U}(1)$}

Let us describe how one can break the $S U(5)$ GUT via a line bundle $L_{Y}$ whose structure group is embedded into $U(1)_{Y}$. This method was used in the context of local F-theory GUT models in [16]. Here we will discuss its implementation within the perturbative orientifold and find important quantisation constraints on the bundle $L_{Y}$. Clearly these have to be taken into account in a string theoretically consistent framework.

Suppose we have designed the model such that the $S U(5)$ gauge symmetry is supported on a D7-brane wrapping a rigid divisor, which is a del Pezzo surface $\mathrm{dP}_{r} \subset Y$ containing $r+1$ homological 2-cycles. Therefore, even though we cannot turn on (discrete) Wilson lines (as $\pi^{1}(D)$ is vanishing), we have the chance to break the $S U(5)$ gauge symmetry to the Standard Model by turning on non-vanishing flux in $U(1)_{Y}$. This Abelian flux $F_{Y}$ is embedded into the fundamental representation of $S U(5)$ as $F_{Y} T_{Y} \subset S U(5)$ with

$$
T_{Y}=\left(\begin{array}{lllll}
-2 & & & & \\
& -2 & & & \\
& & -2 & & \\
& & & 3 & \\
& & & & 3
\end{array}\right) .
$$

Such gauge flux through a non-trivial 2-cycle in $Y$ would lead, via the Green-Schwarz mechanism, to a mass term by mixing with an axion. However for flux supported on a two-cycle of the $\mathrm{dP}_{r}$ trivial in $Y$, there is no axion to pair with and the $U(1)_{Y}$ remains massless after gauge symmetry breaking [30,16]. As discussed in Subsection 2.1, this means that for $U(1)_{Y}$ to remain massless we have to choose $U(1)_{Y}$ such that its first Chern class $c_{1}\left(L_{Y}\right) \in H^{2}\left(D_{a}, \mathbb{Q}\right)$ is trivial on the ambient Calabi-Yau space $Y$, that is, the element $d_{Y} \in H_{2}\left(D_{a}, \mathbb{Q}\right)$ specifying $L_{Y}=\mathcal{O}_{D_{a}}\left(d_{Y}\right)$ must lie in the kernel of the pushforward $\iota_{*} H_{2}\left(D_{a}\right) \rightarrow H_{2}(Y)$. From the relations eq. (5) it is immediately clear that this flux does not change the chiral spectrum, the D7- and D5-tadpole constraints and the Fayet-Iliopoulos terms.

Cancellation of the Freed-Witten anomaly again constrains the quantisation of the bundles $L_{a}$ and $L_{Y}$. In view of the diagonal embedding of $L_{a}$ into $U(5)$ condition eq. (9) becomes

$$
T_{0}\left(c_{1}\left(L_{a}\right)-\iota^{*} B\right)+T_{Y} c_{1}\left(L_{Y}\right)+\frac{1}{2} T_{0} c_{1}\left(K_{D_{a}}\right) \in H^{2}\left(D_{a}, \mathbb{Z}\right)_{5 \times 5}
$$


with $T_{0}=1_{5 \times 5}$. This equation has two important consequences: First, $c_{1}\left(L_{a}\right)-\iota^{*} B$ and $c_{1}\left(L_{Y}\right)$ can take fractional values. For example, for a Spin divisor the choice $c_{1}\left(L_{a}\right)-\iota^{*} B=\frac{2}{5}, c_{1}\left(L_{Y}\right)=\frac{1}{5}$ would be consistent. Second, the two independent conditions encoded in the matrix valued equation (54) cannot be satisfied simultaneously for non-spin divisors without turning on non-trivial flux $F_{a}$ as well14.

The breaking of $S U(5)$ by means of $L_{Y}$ flux induces the standard splitting of the GUT multiplets into MSSM representations,

$$
\begin{aligned}
& \mathbf{2 4} \rightarrow(\mathbf{8}, \mathbf{1})_{0_{Y}}+(\mathbf{1}, \mathbf{3})_{0_{Y}}+(\mathbf{1}, \mathbf{1})_{0_{Y}}+(\mathbf{3}, \mathbf{2})_{5_{Y}}+(\overline{\mathbf{3}}, \mathbf{2})_{-_{5_{Y}}}, \\
& \overline{\mathbf{5}} \rightarrow(\overline{\mathbf{3}}, \mathbf{1})_{2_{Y}}+(\mathbf{1}, \mathbf{2})_{-_{Y}}, \\
& \mathbf{1 0} \rightarrow(\mathbf{3}, \mathbf{2})_{1_{Y}}+(\overline{\mathbf{3}}, \mathbf{1})_{-_{Y}}+(\mathbf{1}, \mathbf{1})_{6_{Y}} \\
& \mathbf{5}_{H} \rightarrow(\mathbf{3}, \mathbf{1})_{-_{Y}}+(\mathbf{1}, \mathbf{2})_{3_{Y}}, \quad \overline{\mathbf{5}}_{H} \rightarrow(\overline{\mathbf{3}}, \mathbf{1})_{2_{Y}}+(\mathbf{1}, \mathbf{2})_{-3_{Y}}
\end{aligned}
$$

As is familiar from the analogous embedding of $\mathrm{U}(1)$ bundles in heterotic compactifications [32] the number of massless states after GUT symmetry breaking is computed by dressing the bundles appearing in the cohomology groups by a factor of $L_{Y}^{q}$. Here $q$ denotes the hypercharge of the MSSM fields.

From the decomposition of the adjoint of $S U(5)$ one deduces that $H^{*}\left(D_{a}, L_{Y}^{ \pm 5}\right)$ gives rise to extra massless states. Clearly, these vector-like exotics are phenomenologically unappealing, so we require that these cohomology groups vanish. This is a very strong requirement and for fifth powers of integer quantised line-bundles on $\mathrm{dP}_{r}$ impossible to satisfy.

However, as discussed above, $c_{1}\left(L_{Y}\right)$ can really take values in $\mathbb{Z} / 5$. To illustrate this further one can modify the embedding as follows. Instead of embedding the line bundle $L_{a}$ entirely into the diagonal $U(1)_{a}$ of $U(5)$ as in eq. (42), one defines two line bundles $\mathcal{L}_{a}$ and $\mathcal{L}_{Y}$ and identifies their field strengths with the following combinations of generators $T_{a}$ and $T_{Y}$ of the diagonal $U(1)_{a}$ and hypercharge $U(1)_{Y}$,

$$
\begin{aligned}
\mathcal{L}_{a} & \leftrightarrow T_{a}, \\
\mathcal{L}_{Y} & \leftrightarrow \frac{2}{5} T_{a}+\frac{1}{5} T_{Y} .
\end{aligned}
$$

\footnotetext{
${ }^{14}$ Note that this conclusion holds also in the context of F-theory compactifications. In a globally consistent setup, non-trivial $L_{Y}$ cannot be switched on at will, but only in combination with nontrivial and suitably quantised 4 -form flux that takes the role of $L_{a}$.
} 
The analogue of condition eq. (154),

$$
\begin{aligned}
& c_{1}\left(\mathcal{L}_{a}\right)-\iota^{*} B+\frac{1}{2} K_{D_{a}} \in \mathbb{Z}, \\
& c_{1}\left(\mathcal{L}_{a}\right)+c_{1}\left(\mathcal{L}_{Y}\right)-\iota^{*} B+\frac{1}{2} K_{D_{a}} \in \mathbb{Z},
\end{aligned}
$$

leads to $c_{1}\left(\mathcal{L}_{Y}\right) \in \mathbb{Z}$ and in general half-integer quantised $\mathcal{L}_{a}$ bundles. This agrees with the fact that all cohomology groups involve integer powers of $\mathcal{L}_{a}$ and $\mathcal{L}_{Y}$. It is important to realise that the gauge flux $U(1)_{Y}$, though being trivial in the cohomology on $Y$ nevertheless does contribute to the D3-tadpole condition. The contribution of the fluxes $L_{a}$ and $L_{Y}$ reads

$$
N_{\text {gauge }}=-\frac{5}{2} \int_{D_{a}} c_{1}^{2}\left(L_{a}\right)-15 \int_{D_{a}} c_{1}^{2}\left(L_{Y}\right)
$$

where we have taken into account $\operatorname{tr}\left(T_{Y}^{2}\right)=30$. Redefining as above $L_{a}=\mathcal{L}_{a} \otimes \mathcal{L}_{Y}^{\frac{2}{5}}$ and $L_{Y}=\mathcal{L}_{Y}^{\frac{1}{5}}$ yields

$$
N_{\text {gauge }}=-\frac{5}{2} \int_{D_{a}} c_{1}^{2}\left(\mathcal{L}_{a}\right)-\int_{D_{a}} c_{1}^{2}\left(\mathcal{L}_{Y}\right)-2 \int_{D_{a}} c_{1}\left(\mathcal{L}_{a}\right) c_{1}\left(\mathcal{L}_{Y}\right)
$$

$\mathcal{L}_{Y}$ being trivial on the Calabi-Yau, the mixed term $\int c_{1}\left(\mathcal{L}_{a}\right) c_{1}\left(\mathcal{L}_{Y}\right)$ may be nonvanishing only if also $\mathcal{L}_{a}$ has components trivial on $Y$.

Let us discuss the effect of $\mathcal{L}_{Y}$ in more detail:

\section{Massless $\mathrm{U}(\mathbf{1})_{\mathbf{Y}}$}

Since we have now embedded the line bundle $\mathcal{L}_{Y}$ into a combination of $T_{0}$ and $T_{Y}$, one might be worried that due to the Green-Schwarz mechanism it is not directly $U(1)_{Y}$ which remains massless. To find the massive Abelian gauge symmetry, we have to evaluate the relevant axion coupling

$$
\int_{\mathbb{R}_{1,3} \times Y} C_{4} \wedge \operatorname{Tr}\left(F_{\mathrm{GUT}}^{2}\right)
$$

where $F_{\mathrm{GUT}}$ is the total $U(5)$ field strength supported on the GUT brane stack. Splitting this into the four-dimensional parts $F^{4 D}$ and the internal background values given by the first Chern classes, we can write

$$
F_{\mathrm{GUT}}=T_{0}\left(F_{0}^{4 D}+c_{1}\left(\mathcal{L}_{a}\right)-\iota^{*} B+\frac{1}{2} c_{1}\left(K_{D_{a}}\right)+\frac{2}{5} c_{1}\left(\mathcal{L}_{Y}\right)\right)+T_{Y}\left(F_{Y}^{4 D}+\frac{1}{5} c_{1}\left(\mathcal{L}_{Y}\right)\right) .
$$


Inserting this into (60) and extracting the relevant term with two legs of $\operatorname{Tr}\left(F_{\text {GUT }}^{2}\right)$ in the four-dimensional Minkowski space and two legs on the GUT D7-brane, one immediately realises that it is still the diagonal $F_{0}^{4 D}$ which mixes with the axions.

\section{Exotics}

As already described the decomposition of the adjoint of $S U(5)$ yields massless states counted by the cohomology groups $H^{*}\left(D_{a}, \mathcal{L}_{Y}\right)$. For phenomenological reasons we require that these cohomology groups vanish. This gives already a very strong constraint on the possible line bundles.

\section{MSSM matter fields}

Using the bundles $\mathcal{L}_{a}$ and $\mathcal{L}_{Y}$, we now express the relevant cohomology groups counting the number quarks and lepton fields. As mentioned these modes localise either on surfaces or on curves. To treat both cases simultaneously we express the number of modes in terms of sheaf extension groups. It is understood that for the actual computation one uses the formulae collected in Subsection 2.3.

The anti-fundamental matter representation of $S U(5)$ splits as

$$
\begin{aligned}
& (\overline{\mathbf{3}}, \mathbf{1})_{2_{Y}}: \operatorname{Ext}^{*}\left(\mathcal{L}_{a}, \mathcal{L}_{b}^{-1}\right) \\
& (\mathbf{1}, \mathbf{2})_{-3_{Y}}: \operatorname{Ext}^{*}\left(\mathcal{L}_{a} \otimes \mathcal{L}_{Y}, \mathcal{L}_{b}^{-1}\right)
\end{aligned}
$$

with $\mathcal{L}_{b}=L_{b}$. Similarly, for the anti-symmetric representation, we have to compute the three cohomology classes

$$
\begin{array}{ll}
(\mathbf{3}, \mathbf{2})_{1_{Y}} & : \operatorname{Ext}^{*}\left(\mathcal{L}_{a}^{-1} \otimes \mathcal{L}_{Y}^{-1}, \mathcal{L}_{a}\right) \\
(\overline{\mathbf{3}}, \mathbf{1})_{4_{Y}} & : \operatorname{Ext}^{*}\left(\mathcal{L}_{a}^{-1}, \mathcal{L}_{a}\right) \\
(\mathbf{1}, \mathbf{1})_{6_{Y}} & : \operatorname{Ext}^{*}\left(\mathcal{L}_{a}^{-1} \otimes \mathcal{L}_{Y}^{-2}, \mathcal{L}_{a}\right)
\end{array}
$$

Since $\mathcal{L}_{Y}$ is trivial in $Y$ it is guaranteed that the chiral index of these representations does not change. However, in general the vector-like matter will change and will be different for MSSM states descending from the same GUT multiplet. This is avoidable if all matter is localised on curves (and not on surfaces) and that the restriction of $\mathcal{L}_{Y}$ to this matter curve vanishes. 


\section{Higgs field and 3-2 splitting}

The fundamental representation for the Higgs field of $S U(5)$ splits as

$$
\begin{array}{ll}
(\mathbf{3}, \mathbf{1})_{-2_{Y}} & : \operatorname{Ext}^{*}\left(\mathcal{L}_{a}^{-1}, \mathcal{L}_{b}^{-1}\right) \\
(\mathbf{1}, \mathbf{2})_{3_{Y}} & : \operatorname{Ext}^{*}\left(\mathcal{L}_{a}^{-1} \otimes \mathcal{L}_{Y}^{-1}, \mathcal{L}_{b}^{-1}\right)
\end{array}
$$

Note that all these states are vector-like. We need that the $S U(2)$ doublets remain massless and that the $S U(3)$ triplets get a mass of the GUT scale. This translates into requiring that $\operatorname{Ext}^{*}\left(\mathcal{L}_{a}^{-1}, \mathcal{L}_{b}^{-1}\right)=(0,0,0,0)$ and $\operatorname{Ext}^{*}\left(\mathcal{L}_{a}^{-1} \otimes \mathcal{L}_{Y}^{-1}, \mathcal{L}_{b}^{-1}\right)=(0,1,1,0)$. For the Higgs fields localised on the intersection curve $C_{a b}$ of the $U(5)$ divisor $D_{a}$ and the $U(1)$ divisor $D_{b}$, two possibilities can occur.

The first option is that the intersection locus is a single elliptic curve, that is, $C_{a b}=T^{2}$. In this case the restriction of the line bundles to $C_{a b}$ have to be of degree zero so that indeed no chiral matter is localised on $C_{a b}$. Recall that a degree zero line bundle on the elliptic curve $C_{a b}$ can be written as $\mathcal{O}(p-q)$, where $p, q$ denote points different from the origin 0 of the elliptic curve. The trivial bundle $\mathcal{O}$ corresponds to $p-q=0$ and has cohomology $H^{*}\left(C_{a b}, \mathcal{O}\right)=(1,1)$. If $p-q \neq 0$ the line bundle has a non-trivial Wilson line and the cohomology groups vanish. It is therefore clear that for an appropriate choice of the line bundles appearing in eq. (64) it can be arranged that only the doublet remains massless while the triplets acquire string scale masses. According to what we just said this happens provided the restriction of the line bundles appearing in eq. (64) to the genus one matter curve $C_{a b}$ take the form

$$
\begin{aligned}
& \left.\mathcal{L}_{a}^{-1} \otimes \mathcal{L}_{b}\right|_{C_{a b}}=\mathcal{O}(p-q), \quad p-q \neq 0 \\
& \left.\mathcal{L}_{a}^{-1} \otimes \mathcal{L}_{Y}^{-1} \otimes \mathcal{L}_{b}\right|_{C_{a b}}=\mathcal{O}
\end{aligned}
$$

To see how to arrange for this, suppose one has found a model where the line bundles $\mathcal{L}_{a}$ and $\mathcal{L}_{b}$ can both be written as the pullback of line bundles from the Calabi-Yau,

$$
\mathcal{L}_{a}=\iota_{a}^{*} \mathbb{L}_{a}, \quad \mathcal{L}_{b}=\iota_{b}^{*} \mathbb{L}_{b}
$$

Since $\left.\mathcal{L}_{a}^{-1} \otimes \mathcal{L}_{b}\right|_{C_{a b}}$ is of degree zero in this case $\left.\mathcal{L}_{a}^{-1} \otimes \mathcal{L}_{b}\right|_{C_{a b}}=\mathcal{O}$ with trivial Wilson lines. The relations eq. (65) can now simply be met by twisting $\mathcal{L}_{a}$ by a line bundle $R_{a}$ on $D_{a}$ which is trivial on the ambient manifold and satisfies

$$
R_{a}=\mathcal{L}_{Y}^{-1}
$$


As with everything desirable in life this surgery is not for free, as the new contribution of the GUT brane to the D3-brane tadpole is increased from eq. (59) td 15

$$
N_{\text {gauge }}^{a}=-\frac{5}{2} \int_{D_{a}} c_{1}^{2}\left(\mathcal{L}_{a}\right)-\frac{3}{2} \int_{D_{a}} c_{1}^{2}\left(\mathcal{L}_{Y}\right)
$$

We will however also encounter caset 16 in which eq. (66) is not the situation to begin with. In particular it may be inconsistent to define the final GUT bundle as $\iota_{a}^{*} \mathbb{L}_{a} \otimes \mathcal{L}_{Y}^{-1}$ because this bundle might exhibit ghosts in its spectrum. For the line bundle appearing in (64), in this case we have, supposing for definiteness that $\mathcal{L}_{b}=\iota_{b}^{*} \mathbb{L}_{b}$,

$$
\left.\mathcal{L}_{a}^{-1} \otimes \mathcal{L}_{Y}^{-1} \otimes \mathcal{L}_{b}\right|_{C_{a b}}=\mathcal{O}\left(p_{1}-q_{1}\right)
$$

We then need to twist $\mathcal{L}_{b}$ by a line bundle $R_{b}$ trivial on the ambient space, and every such bundle satisfies $\left.R_{b}\right|_{C_{a b}}=\left.\mathcal{O}\left(p_{2}-q_{2}\right)\right|_{C_{a b}}$. To ensure $p_{1}+p_{2}-\left(q_{1}+q_{2}\right)=0$, as desired, might require adjusting some of the complex structure moduli of the manifold.

On the other hand, it was argued in [16] that in case $H_{u}$ and $H_{d}$ are localised on a single curve, dimension five proton decay operators $Q Q Q L$ can be generated by exchanging Kaluza-Klein modes of the Higgs-triplet. To avoid such operators, it was suggested that the intersection locus $D_{a} \cap D_{b}$ consists of two components $C_{1} \cup C_{2}$, such that the $5_{H}$ originates from $H^{*}\left(C_{1}, \mathcal{L}_{a}^{-1} \otimes \mathcal{L}_{b} \otimes \sqrt{K_{C_{1}}}\right)=(1,0)$ and $\overline{\boldsymbol{5}}_{H}$ from $H^{*}\left(C_{2}, \mathcal{L}_{a}^{-1} \otimes \mathcal{L}_{b} \otimes \sqrt{K_{C_{1}}}\right)=(0,1)$. This is the second option we have for the localisation of the Higgs field.

\section{The top Yukawa couplings}

We have seen that in the $S U(5)$ GUT model the top quark Yukawa coupling $1010 \mathbf{5}_{\mathbf{H}}$ can be generated by a single rigid $O(1)$ instanton with the right charged matter zero mode structure. We need to check whether this is compatible with the breaking of the $S U(5)$ GUT group by the $U(1)_{Y}$ flux.

Recall that the five zero modes $\lambda_{a}^{i}$ transform in the $\overline{\mathbf{5}}$ representation of the $S U(5)$ and the single zero mode $\lambda_{b}$ in the singlet representation of $S U(5)$ with $U(1)_{b}$ charge $q_{b}=+1$. The $U(1)_{Y}$ flux splits the $\overline{\mathbf{5}}$ representation according to (55), that is,

\footnotetext{
${ }^{15}$ Here we are using that $\mathcal{L}_{a}=\iota_{a}^{*} \mathbb{L}_{a}$ prior to twisting by $R_{a}$ so that the cross-term in eq. (59) vanishes.

${ }^{16}$ This discussion is only relevant for the models proposed in Section 5 and Subsection 6.1.
} 
the $(\overline{\mathbf{3}}, \mathbf{1})_{2_{Y}}$ zero modes are counted by $\operatorname{Ext}^{*}\left(\mathcal{L}_{a}, \mathcal{O}\right)$ and the $(\overline{\mathbf{1}}, \mathbf{2})_{-3_{Y}}$ modes by $\operatorname{Ext}^{*}\left(\mathcal{L}_{a} \otimes \mathcal{L}_{Y}, \mathcal{O}\right)$. As long as the $S U(5)$ stack of branes and the instanton brane intersect over a 2-cycle non-trivial in the Calabi-Yau manifold, the restriction of $\mathcal{L}_{Y}$ to the intersection locus vanishes and we get precisely three instanton zero modes $\lambda_{a}^{i}$ transforming in the $(\overline{\mathbf{3}}, \mathbf{1})_{2_{Y}}$ representation and two zero modes $\tilde{\lambda}_{a}^{j}$ transforming in the $(\overline{\mathbf{1}}, \mathbf{2})_{-3_{Y}}$ representation. Since $\mathcal{L}_{Y}$ is supported on the $S U(5)$ stack, the single zero mode $\lambda_{b}$ also still exists. Then the Standard Model top-Yukawa couplings $(\mathbf{3}, \mathbf{2})(\overline{\mathbf{3}}, \mathbf{1})(\mathbf{1}, \mathbf{2})_{H}$ are generated by the instanton via the following absorption of the six charged instanton zero modes

$$
\begin{array}{c|l}
(\mathbf{3}, \mathbf{2})_{1_{Y}} & (\overline{\mathbf{3}}, \mathbf{1})_{2_{Y}}^{\lambda_{a}}(\mathbf{1}, \mathbf{2})_{-3_{Y}}^{\tilde{\lambda}_{a}} \\
(\overline{\mathbf{3}}, \mathbf{1})_{-4_{Y}} & (\overline{\mathbf{3}}, \mathbf{1})_{2_{Y}}^{\lambda_{a}}(\overline{\mathbf{3}}, \mathbf{1})_{2_{Y}}^{\lambda_{a}} \\
(\mathbf{1}, \mathbf{2})_{3_{Y}} & (\mathbf{1}, \mathbf{2})_{-3_{Y}}^{\tilde{\lambda}_{a}}(\mathbf{1}, \mathbf{1})_{0_{Y}}^{\lambda_{b}}
\end{array}
$$

\section{The $\mu$-term}

The supersymmetric $\mu$ term clearly vanishes at tree-level. For having it directly generated non-perturbatively, the rigid $O(1)$ instanton must carry the four charged matter zero modes $\lambda_{a}, \bar{\lambda}_{a}$ and $\lambda_{b}, \bar{\lambda}_{b}$. However, due to the $S U(2)$ Chan-Paton factor the $\lambda$ always come in multiples of two, so that these simple non-perturbative $\mu$ terms are absent. However, they can be generated by higher dimensional operators involving $S U(5)$ gauge singlets, which have to receive some non-vanishing vacuum expectation value.

\subsection{Summary of GUT Model Building Constraints}

In this section we have collected a number of perturbative and non-perturbative stringy mechanisms to first realise Georgi-Glashow $S U(5)$ GUTs and second to solve some of their inherent problems. The perfect string model, besides being globally consistent would of course satisfy all these constraints. Eventually, one also has to address the issue of moduli stabilisation by fluxes and instantons, some aspects of which we discuss in Section 8. A more thorough and complete analysis is beyond the main scope of this paper but truly on the agenda.

Let us summarise in Table 3 the main properties a realistic string GUT model should have 


\begin{tabular}{|c||c|c|}
\hline \hline property & mechanism & status \\
\hline \hline globally consistent & tadpoles + K-theory & $\checkmark$ \\
D-term susy & vanishing FI-terms inside Kähler cone & $\checkmark$ \\
gauge group $S U(5)$ & choice of line bundles $\mathcal{L}_{a, b}$ & $\checkmark$ \\
3 chiral generations & localisation on curves & $\checkmark$ \\
no vector-like matter & choice of line bundles & $\checkmark$ \\
1 vector-like of Higgs & rigid 4 -cycles $\leftarrow$ del Pezzo & $\checkmark$ \\
no adjoints & $U(1)_{Y}$ flux $\mathcal{L}_{Y}$ on trivial 2-cycles & $\checkmark$ \\
GUT breaking & Wilson lines on $g=1$ curve & $\checkmark$ \\
$3-2$ splitting & local. of $H_{u}, H_{d}$ on disjoint comp. & $\checkmark$ \\
3 split + no dim=5 $p^{+}$-decay & perturb. or D3-instanton & $\checkmark$ \\
$\mathbf{1 0} \overline{\mathbf{5}}_{H}$ Yukawa & presence of appropriate D3-instanton & $\checkmark$ \\
$\mathbf{1 0} \mathbf{1 0} \mathbf{5}_{H}$ Yukawa & presence of appropriate D3-instanton & $\checkmark$ \\
\hline Majorana neutrino masses & & \\
\hline \hline
\end{tabular}

Table 3: Summary of SU(5) properties and their realisations by different Type IIB orientifold mechanisms. The mark $\checkmark$ in the last column indicates that all features can in principle be realised. 


\section{Del Pezzo Transitions on $\mathbb{P}_{1,1,1,6,9}[18]$}

In this section we discuss a first class of Calabi-Yau orientifold backgrounds which will later support our GUT models. The underlying geometries are compact CalabiYau manifolds $M_{n}$ which can be either constructed as elliptic fibrations over del Pezzo surfaces $\mathrm{dP}_{n}$, or by performing del Pezzo transitions. To set the stage for our analysis of the Calabi-Yau orientifolds we will first recall some basic geometric facts about the surfaces $\mathrm{dP}_{n}$ in Subsection 4.1 and classify all involutions on these surfaces. In Subsection 4.2 we argue that the elliptically fibred Calabi-Yau threefolds over $\mathrm{dP}_{n}$ can also be obtained by del Pezzo transitions starting from the degree 18 hypersurface in $\mathbb{P}_{1,1,1,6,9}$ after flop transitions. Simple examples are obtained if the base is one of the toric del Pezzo surfaces $\mathrm{dP}_{2}$ or $\mathrm{dP}_{3}$. In Sections 4.3 and 4.4 we construct the corresponding Calabi-Yau threefolds and study their different topological phases using toric geometry. In a second step we introduce viable orientifold involutions $\sigma$ on these compact Calabi-Yau manifolds and derive the induced tadpoles from $\mathrm{O} 7$ and O3 planes.

In the second part of this section we will have a closer look at the topological phases of $M_{n}$ with $n \mathrm{dP}_{8}$ surfaces. We show in Subsection 4.5 that these are examples of so-called swiss-cheese Calabi-Yau manifolds which can support large volume vacua with one large and $n+1$ small four-cycles [7, 8, 66]. In Subsection 4.6 we discuss the D-term conditions arising from wrapping a D7-brane on the small del Pezzo surfaces with orientifold invariant homology class.

\subsection{Del Pezzo Surfaces and Their Involutions}

The compact orientifold geometries for our GUT models will be obtained from elliptic fibrations over del Pezzo surfaces $\mathrm{dP}_{n}$. In order to study these threefolds it will be necessary to review some basic facts about del Pezzo surfaces first. We will also discuss involutions on these $\mathrm{dP}_{n}$. We will determine their fix-point locus and action on the exceptional curves of the del Pezzo surface. Since these involutions on the base will descend to involutions on the entire Calabi-Yau manifold, this will enable us to identify viable brane configurations later on. 


\section{On the geometry of del Pezzo surfaces}

By definition, del Pezzo surfaces are the Fano surfaces, that is, the algebraic surfaces with ample canonical bundle. These are either the surfaces $\mathcal{B}_{n}=\mathrm{dP}_{n}$, which are obtained by blowing up $\mathbb{P}^{2}$ on $0 \leq n \leq 8$ point 17 , or $\mathbb{P}^{1} \times \mathbb{P}^{1}$. Their Hodge numbers are $h^{0,0}=h^{2,2}=1, h^{1,1}\left(\mathcal{B}_{n}\right)=n+1$ and $h^{1,1}\left(\mathbb{P}^{1} \times \mathbb{P}^{1}\right)=2$, while all other $h^{p, q}$ vanish. A basis of homologically nontrivial two-cycles in $\mathcal{B}_{n}$ consists of the class of a line $l$ in $\mathbb{P}^{2}$, and the $n$ exceptional curves $e_{i}$, one for each blown-up point. Their intersection numbers are $l^{2}=1, e_{i} \cdot e_{j}=-\delta_{i j}, e_{i} \cdot l=0$. Written in this basis, the first Chern class is

$$
c_{1}\left(T \mathcal{B}_{n}\right)=-K=3 l-\sum_{i=1}^{n} e_{i}
$$

The square of the canonical class

$$
K^{2}=\int_{\mathcal{B}_{n}} c_{1}^{2}=9-n
$$

is also called the degree 18 of the del Pezzo surface. The second (top) Chern class is the Euler density, hence

$$
\chi\left(\mathcal{B}_{n}\right)=\int_{\mathcal{B}_{n}} c_{2}=3+n .
$$

Let $C$ be a curve in the del Pezzo surface. Then its degree $\operatorname{deg}(C)$ and its arithmetic genus $g$ read

$$
\operatorname{deg}(C)=-K \cdot C, \quad g=\frac{1}{2}(C \cdot C+K \cdot C)+1 .
$$

Of particular interest are the rigid genus-0 instantons, that is rational curves of selfintersection $(-1)$. For convenience of the reader we reproduce the classification of such (-1)-curves, see [67, in Table 4.

The del Pezzo surfaces $\mathbb{P}^{2}=\mathcal{B}_{0}, \mathbb{P}^{1} \times \mathbb{P}^{1}, \mathcal{B}_{1}, \mathcal{B}_{2}$, and $\mathcal{B}_{3}$ (that is, those of degree $K^{2} \geq 6$ ) are toric varieties. The remaining surfaces $\mathcal{B}_{4}, \ldots, \mathcal{B}_{8}$ are not toric

\footnotetext{
${ }^{17}$ The points must be general in the sense that no two points are infinitesimally close, no three are on one line, no six on a conic, no eight on a cubic with a node at one of them. In other words, one is not allowed to blow up points sitting on a $(-1)$-curve. If one were to blow up a point on a $(-1)$-curve, then the proper transform would be a (-2)-curve. So yet another characterisation of the allowed points is that there be no curves of self-intersection -2 or less. Moreover, note that different sets of points can correspond to the same (complex structure on the) del Pezzo surface.

${ }^{18}$ To understand this notation, note that a degree $d=K^{2}$ del Pezzo surface with $d \geq 3$ can be realised as a degree- $d$ hypersurface in $\mathbb{P}^{d}$.
} 


\begin{tabular}{|c|rrrrrrrr|}
\hline class $\in H_{2}\left(\mathcal{B}_{n}, \mathbb{Z}\right)$ & $\mathcal{B}_{1}$ & $\mathcal{B}_{2}$ & $\mathcal{B}_{3}$ & $\mathcal{B}_{4}$ & $\mathcal{B}_{5}$ & $\mathcal{B}_{6}$ & $\mathcal{B}_{7}$ & $\mathcal{B}_{8}$ \\
\hline$(0 ; 1)$ & 1 & 2 & 3 & 4 & 5 & 6 & 7 & 8 \\
$\left(1 ;-1^{2}\right)$ & & 1 & 3 & 6 & 10 & 15 & 21 & 28 \\
$\left(2 ;-1^{5}\right)$ & & & & & 1 & 6 & 21 & 56 \\
$\left(3 ;-2,-1^{6}\right)$ & & & & & & & 7 & 56 \\
$\left(4 ;-2^{3},-1^{5}\right)$ & & & & & & & & 56 \\
$\left(5 ;-2^{6},-1^{2}\right)$ & & & & & & & & 28 \\
$\left(6 ;-3,-2^{7}\right)$ & & & & & & & & 8 \\
\hline Total no. & 1 & 3 & 6 & 10 & 16 & 27 & 56 & 240 \\
\hline
\end{tabular}

Table 4: Number of $(-1)$-curves on the $\mathcal{B}_{n}$ del Pezzo surfaces. The coefficients $\left(a ; b_{1}, \ldots b_{n}\right)$ are with respect to the standard basis $\left(l ; e_{1}, \ldots, e_{n}\right)$. For example, $\left(1,-1^{2}\right)$ denotes all $\left(\begin{array}{l}n \\ 2\end{array}\right)$ homology classes of the form $l-e_{i}-e_{j}, 1 \leq i<j \leq n$. Note that there are no $(-1)$-curves on $\mathcal{B}_{0}=\mathbb{P}^{2}$ and $\mathbb{P}^{1} \times \mathbb{P}^{1}$, which are omitted.

varieties, but can of course be embedded into toric varieties. In particular, the del Pezzo surfaces $\mathcal{B}_{5}, \ldots, \mathcal{B}_{8}$ are hypersurfaces or complete intersections in weighted projective spaces. For this, let us denote by $\mathbb{P}\left(d_{1}, \ldots, d_{r} \mid w_{0}, \ldots, w_{m}\right)$ the complete intersection of $r$ equations of homogeneous degree $d_{1}, \ldots, d_{r}$ in weighted projective space with weights $w_{0}, \ldots, w_{m}$. These del Pezzo surfaces are listed in Table 5, One infers that the dimension of the complex deformation spaces for del Pezzo surfaces $\mathcal{B}_{n}$ with $n \geq 5$ is $\operatorname{dim} H^{1}\left(T \mathcal{B}_{n}\right)=2 n-8$.

\begin{tabular}{|c|c|c|l|}
\hline del Pezzo & $K^{2}$ & hypersurface & coordinates \\
\hline $\mathcal{B}_{5}$ & 4 & $\mathbb{P}(2,2 \mid 1,1,1,1,1)$ & $\left(x_{1}, x_{2}, x_{3}, x_{4}, x_{5}\right)$ \\
$\mathcal{B}_{6}$ & 3 & $\mathbb{P}(3 \mid 1,1,1,1)$ & $\left(x_{1}, x_{2}, x_{3}, x_{4}\right)$ \\
$\mathcal{B}_{7}$ & 2 & $\mathbb{P}(4 \mid 2,1,1,1)$ & $\left(y, x_{1}, x_{2}, x_{3}\right)$ \\
$\mathcal{B}_{8}$ & 1 & $\mathbb{P}(6 \mid 3,2,1,1)$ & $\left(y, z, x_{1}, x_{2}\right)$ \\
\hline
\end{tabular}

Table 5: The del Pezzo surfaces of degree $K^{2} \leq 4$. 


\section{Classification of involutions on del Pezzo surfaces}

In order to systematically study GUT models on elliptically fibred Calabi-Yau manifolds with del Pezzo base, one needs to classify all different, non-trivial, holomorphic involutions on del Pezzo surfaces. In the following we intend to discuss the final classification in Table 6 and give a first impression of the necessary steps needed for this derivation. Most of the technical details and geometric constructions are shifted into Appendix A. The classification in Table 6 completes the list obtained in ref. [41].

For a systematic classification of involutions we will look at the pattern of rigid $\mathbb{P}^{1}$ instantons, that is, the $(-1)$-curves. Clearly, every involution induces a $\mathbb{Z}_{2}$ automorphism of the $(-1)$-curves. Conversely, up to degree 6 , the automorphism of the $(-1)$-curves determines the involution. In the remaining degrees $\geq 7$ there either are no $(-1)$-curves, or they lie over a line or point of the blown-up $\mathbb{P}^{2}$. Hence, in the latter case they do not "fill out" the space to uniquely determine the involution. Technically, the $(-1)$-curves generate all of $H_{2}(S, \mathbb{Z})$ if and only if the degree is 6 or less. In a next step, one has to find all conjugacy classes of involutions acting on the $(-1)$ curves and check that these descend to actual geometric involutions on the corresponding del Pezzo surface. The details of this analysis can be found in Appendix A. Here we will discuss the final classification summarised in Table 6.

In Table 6 the complete list of del Pezzo surfaces $\mathcal{B}_{n}$ with involutions $\sigma_{i}$ is shown. For each pair $\left(\mathcal{B}_{n}, \sigma_{i}\right)$ it also includes detailed information about the fix-point set. We use the following notation:

- $\Sigma(\sigma)$ is the homology class of the genus- $g$ curve with $g \geq 1$ in the fixed point set of $\sigma$. As discussed in Appendix A, there is at most one such curve.

- $R(\sigma)$ are the homology classes of the rational genus 0 curves in the fixed point set.

- $B(\sigma)$ is the number of isolated fixed points that do not lie on $(-1)$-curves.

- $P(\sigma)$ is the number of isolated fixed points that do lie on $(-1)$-curves, and hence may not be blown up further.

- $\left(b_{2}^{+}, b_{2}^{-}\right)$are the dimensions of the \pm eigenspaces of $\sigma_{i}$ in $H_{2}\left(\mathcal{B}_{n}\right)$.

In the last column we also displayed the explicit action of the involution on the basis $\left(l, e_{1}, \ldots, e_{n}\right)$ of $H_{2}\left(\mathcal{B}_{n}\right)$ and basis $\left(l_{1}, l_{2}\right)$ of $H_{2}\left(\mathbb{P}^{1} \times \mathbb{P}^{1}\right)$. The $k$-dimensional identity matrix is simply denoted by $\mathbf{1}_{k}$, while $H$ exchanges two elements $e_{i} \leftrightarrow e_{j}$ or $l_{1} \leftrightarrow l_{2}$. 
In addition there are also five involutions $\left(I_{\mathcal{B}_{3}}^{(2)}, I_{\mathcal{B}_{3}}^{(3)}, I_{\mathcal{B}_{5}}^{(5)}, I_{\mathcal{B}_{7}}^{(9)}, I_{\mathcal{B}_{8}}^{(9)}\right)$ which should be viewed as the additional building blocks for all non-trivial involutions on del Pezzo surfaces. We will introduce the explicit form of these involutions in turn.

To define the special involutions we will specify their action on the basis elements $\left(l, e_{1}, \ldots, e_{n}\right)$. On the third del Pezzo surface we define the two involutions

$$
I_{\mathcal{B}_{3}}^{(2)}=\left(\begin{array}{cccc}
2 & 1 & 1 & 1 \\
-1 & -1 & 0 & -1 \\
-1 & 0 & -1 & -1 \\
-1 & -1 & -1 & 0
\end{array}\right), \quad I_{\mathcal{B}_{3}}^{(3)}=\left(\begin{array}{cccc}
2 & 1 & 1 & 1 \\
-1 & 0 & -1 & -1 \\
-1 & -1 & 0 & -1 \\
-1 & -1 & -1 & 0
\end{array}\right) .
$$

The remaining three involutions we need to introduce are well-known classical involutions. They are minimal since they satisfy $\sigma(E) \neq E$ and $\sigma(E) \cap E \neq \emptyset$ for each $(-1)$-curve $E$. This implies that such an involution cannot be obtained by blowing up a higher degree del Pezzo and extending an involution defined on the del Pezzo surface before blow-up. On $\mathcal{B}_{5}$ there is a minimal involution known as the de Jonquières involution which acts as

$$
I_{\mathcal{B}_{5}}^{(5)}=\left(\begin{array}{cccccc}
3 & 2 & 1 & 1 & 1 & 1 \\
-2 & -1 & -1 & -1 & -1 & -1 \\
-1 & -1 & -1 & 0 & 0 & 0 \\
-1 & -1 & 0 & -1 & 0 & 0 \\
-1 & -1 & 0 & 0 & -1 & 0 \\
-1 & -1 & 0 & 0 & 0 & -1
\end{array}\right) .
$$

There is one minimal involution for the del Pezzo surfaces of degree 1 and 2, respectively. The del Pezzo surface $\mathcal{B}_{7}$ admits the Geiser involution

$$
I_{\mathcal{B}_{7}}^{(9)}: \quad l \mapsto-l-3 K, \quad e_{i} \mapsto-K-e_{i} .
$$

while on $\mathcal{B}_{8}$ one has the Bertini involution acting as

$$
I_{\mathcal{B}_{8}}^{(9)}: \quad l \mapsto-l-6 K, \quad e_{i} \mapsto-2 K-e_{i} .
$$

Note that one can explicitly check that each involution on each del Pezzo surface preserves its canonical class $K$ defined in eq. (71).

With these definitions at hand, the involutions in Table 6 can be used for explicit computations. This will be particularly useful for the elliptically fibred threefolds over a del Pezzo base, since all involutions can be lifted to the corresponding Calabi-Yau threefold. We are then in the position to construct explicit Calabi-Yau orientifolds and compute the tadpoles induced by the O3- and O7-planes.

\subsection{The Geometry of Del Pezzo Transitions of $\mathbb{P}_{1,1,1,6,9}[18]$}

We are now in the position to construct compact Calabi-Yau threefolds $M_{n}$ associated to a del Pezzo base. The first construction is via an elliptic fibration over a del Pezzo base $\mathcal{B}_{n}$, while the second construction is by employing del Pezzo transitions. 


\begin{tabular}{|c|c|c|c|c|c|c|c|}
\hline Involution & $g$ & $\Sigma=\left[\Sigma_{g}\right]$ & $R$ & $B$ & $P$ & $\left(b_{2}^{+}, b_{2}^{-}\right)$ & action on $\mathrm{H}_{2}$ \\
\hline$\left(\mathbb{P}^{2}, \sigma\right)$ & & & $l$ & 1 & & $(1,0)$ & $\mathbf{1}_{1}$ \\
\hline$\left(\mathbb{P}^{1} \times \mathbb{P}^{1}, \sigma_{1}\right)$ & & & $\left(l_{1}\right) \cup\left(l_{2}\right)$ & & & $(2,0)$ & $\mathbf{1}_{2}$ \\
\hline$\left(\mathbb{P}^{1} \times \mathbb{P}^{1}, \sigma_{2}\right)$ & & & & 4 & & $(2,0)$ & $\mathbf{1}_{2}$ \\
\hline$\left(\mathbb{P}^{1} \times \mathbb{P}^{1}, \sigma_{3}\right)$ & & & $l_{1}+l_{2}$ & & & $(1,1)$ & $H$ \\
\hline$\left(\mathcal{B}_{1}, \sigma_{1}\right)$ & & & $(l) \cup\left(e_{1}\right)$ & & & $(2,0)$ & $\mathbf{1}_{2}$ \\
\hline$\left(\mathcal{B}_{1}, \sigma_{2}\right)$ & & & $l-e_{1}$ & 1 & 1 & $(2,0)$ & $\mathbf{1}_{2}$ \\
\hline$\left(\mathcal{B}_{2}, \sigma_{1}\right)$ & & & $\left(l-e_{1}\right) \cup\left(e_{2}\right)$ & & 1 & $(3,0)$ & $\mathbf{1}_{3}$ \\
\hline$\left(\mathcal{B}_{2}, \sigma_{2}\right)$ & & & $l-e_{1}-e_{2}$ & 1 & 2 & $(3,0)$ & $\mathbf{1}_{3}$ \\
\hline$\left(\mathcal{B}_{2}, \sigma_{3}\right)$ & & & $l$ & & 1 & $(2,1)$ & $\mathbf{1}_{1} \oplus H$ \\
\hline$\left(\mathcal{B}_{3}, \sigma_{1}\right)$ & & & $\left(l-e_{1}-e_{2}\right) \cup\left(e_{3}\right)$ & & 2 & $(4,0)$ & $\mathbf{1}_{4}$ \\
\hline$\left(\mathcal{B}_{3}, \sigma_{2}\right)$ & & & $l-e_{1}$ & & 2 & $(3,1)$ & $\mathbf{1}_{2} \oplus H$ \\
\hline$\left(\mathcal{B}_{3}, \sigma_{3}\right)$ & & & $2 l-e_{1}-e_{2}$ & & & $(2,2)$ & $I_{\mathcal{B}_{3}}^{(2)}$ \\
\hline$\left(\mathcal{B}_{3}, \sigma_{4}\right)$ & & & & 4 & & $(3,1)$ & $I_{\mathcal{B}_{3}}^{(3)}$ \\
\hline$\left(\mathcal{B}_{4}, \sigma_{1}\right)$ & & & $l-e_{1}-e_{2}$ & & 3 & $(4,1)$ & $\mathbf{1}_{3} \oplus H$ \\
\hline$\left(\mathcal{B}_{4}, \sigma_{2}\right)$ & & & $l$ & & 1 & $(3,2)$ & $\mathbf{1}_{1} \oplus 2 H$ \\
\hline$\left(\mathcal{B}_{5}, \sigma_{1}\right)$ & & & $l-e_{1}$ & & 2 & $(4,2)$ & $\mathbf{1}_{2} \oplus 2 H$ \\
\hline$\left(\mathcal{B}_{5}, \sigma_{2}\right)$ & & & $2 l-e_{1}-e_{2}$ & & & $(3,3)$ & $I_{\mathcal{B}_{3}}^{(2)} \oplus H$ \\
\hline$\left(\mathcal{B}_{5}, \sigma_{3}\right)$ & & & & 4 & & $(4,2)$ & $I_{\mathcal{B}_{3}}^{(3)} \oplus H$ \\
\hline$\left(\mathcal{B}_{5}, \sigma_{\mathrm{dJ}}\right)$ & 1 & $3 l-\sum_{i=1}^{5} e_{i}$ & & & & $(2,4)$ & $I_{\mathcal{B}_{5}}^{(5)}$ \\
\hline$\left(\mathcal{B}_{6}, \sigma_{1}\right)$ & & & $l-e_{1}-e_{2}$ & & 3 & $(5,2)$ & $\mathbf{1}_{3} \oplus 2 H$ \\
\hline$\left(\mathcal{B}_{6}, \sigma_{2}\right)$ & 1 & $3 l-\sum_{i=1}^{6} e_{i}$ & & & 1 & $(3,4)$ & $I_{\mathcal{B}_{5}}^{(5)} \oplus \mathbf{1}_{1}$ \\
\hline$\left(\mathcal{B}_{7}, \sigma_{1}\right)$ & & & & 4 & & $(5,3)$ & $I_{\mathcal{B}_{3}}^{(3)} \oplus 2 H$ \\
\hline$\left(\mathcal{B}_{7}, \sigma_{2}\right)$ & 1 & $3 l-\sum_{i=1}^{7} e_{i}$ & & & 2 & $(4,4)$ & $I_{\mathcal{B}_{5}}^{(5)} \oplus \mathbf{1}_{2}$ \\
\hline$\left(\mathcal{B}_{7}, \sigma_{3}\right)$ & 1 & $3 l-\sum_{i=1}^{5} e_{i}$ & & & & $(3,5)$ & $I_{\mathcal{B}_{5}}^{(5)} \oplus H$ \\
\hline$\left(\mathcal{B}_{7}, \sigma_{\mathrm{G}}\right)$ & 3 & $6 l-2 \sum_{i=1}^{7} e_{i}$ & & & & $(1,7)$ & $I_{\mathcal{B}_{7}}^{(9)}$ \\
\hline$\left(\mathcal{B}_{8}, \sigma_{1}\right)$ & 1 & $3 l-\sum_{i=1}^{8} e_{i}$ & & & 3 & $(5,4)$ & $I_{\mathcal{B}_{5}}^{(5)} \oplus \mathbf{1}_{3}$ \\
\hline$\left(\mathcal{B}_{8}, \sigma_{2}\right)$ & 1 & $3 l-\sum_{i=1}^{6} e_{i}$ & & & 1 & $(4,5)$ & $I_{\mathcal{B}_{5}}^{(5)} \oplus \mathbf{1}_{1} \oplus H$ \\
\hline$\left(\mathcal{B}_{8}, \sigma_{\mathrm{B}}\right)$ & 4 & $9 l-3 \sum_{i=1}^{7} e_{i}$ & & 1 & & $(1,8)$ & $I_{\mathcal{B}_{8}}^{(9)}$ \\
\hline
\end{tabular}

Table 6: All involutions on del Pezzo surfaces. See page 42 for the definition of $\Sigma, R, B$, and $P$. 


\section{Elliptically fibred Calabi-Yau threefolds with del Pezzo base}

Let us construct Calabi-Yau threefolds $M_{n}$ as elliptic fibrations over the del Pezzo base $\mathrm{dP}_{n}$. We consider elliptic fibrations which are generically smooth with the worst degeneration of the fibre of Kodaira type $I_{1}$ [68,69]. In the following we will restrict further to elliptic fibrations with generic elliptic fibres of type $E_{8}$ such that the generic elliptic fibres can be represented by a degree 6 hypersurface in $\mathbb{P}_{1,2,3}$ denoted by $\mathbb{P}_{1,2,3}[6]$. As shown, for example, in [70], one then finds that the Euler number of the elliptic fibration $M_{n}$ is given by

$$
\chi\left(M_{n}\right)=-C_{(8)} \int_{\mathcal{B}_{n}} c_{1}^{2}=60(n-9),
$$

where $C_{(8)}=30$ is the dual Coxeter number of $E_{8}$ and we have used (72) for the del Pezzo base $\mathcal{B}_{n}$. One can also count the number of Kähler classes for these geometries. One finds that there are $n+1$ classes corresponding to the non-trivial two-cycles of the del Pezzo base as well as the fibre class of the elliptic fibration. This implies that $M_{n}$ has Hodge numbers

$$
h^{1,1}\left(M_{n}\right)=n+2, \quad h^{2,1}\left(M_{n}\right)=272-29 n,
$$

where we have used that $\chi=2\left(h^{1,1}-h^{2,1}\right)$.

The specification of $M_{n}$ as an elliptic fibration over the base $\mathrm{dP}_{n}$ will turn out to be particularly useful in the study of orientifold involutions and brane configurations on $M_{n}$. Let us introduce the map

$$
\pi: M_{n} \rightarrow \mathcal{B}_{n},
$$

which is the projection from the threefold $M_{n}$ to the base. Note that every $(-1)$ curve class $E$ in $\mathcal{B}_{n}$ can be pulled back to a divisor in $M_{n}$ using $\pi^{*}: E \mapsto \pi^{*}(E) \in$ $H_{4}\left(M_{n}, \mathbb{Z}\right)$. In fact, each such divisor is a $\mathrm{dP}_{9}$ surface. This surface is defined as blow up of $\mathbb{P}^{2}$ at nine points which arise at the intersections of two cubic curves. Thus, $\mathrm{dP}_{9}$ is an elliptic fibration over $\mathbb{P}^{1}$ which has 12 singular fibres 19 . The $\mathrm{dP}_{9}$ is not strictly a del Pezzo surface, but the equations (172) and (73) remain to be valid. Recall that the $(-1)$ curve in the base have been listed in Table 4, It it thus straightforward to determine the intersections of these curves. In case two curves $E_{1}, E_{2}$ intersect at a point the corresponding two $\mathrm{dP}_{9}$ divisors $\pi^{*}\left(E_{1}\right)$ and $\pi^{*}\left(E_{2}\right)$ in $M_{n}$ will intersect

\footnotetext{
${ }^{19}$ Roughly speaking, the $\mathrm{dP}_{9}$ is half a $\mathrm{K} 3$ surface which is an elliptic fibration over $\mathbb{P}^{1}$ with 24 singular fibres.
} 
over a Riemann surface of genus 1 . Clearly each $\pi^{*}(E)$ intersects the base $\mathcal{B}_{n}$ in a $\mathbb{P}^{1}$. Already this simple analysis allows us to infer the necessary information on the triple intersections of the threefold $\mathcal{M}_{n}$ in the elliptically fibred phase.

We want to apply a similar logic also for the extension of an involution on the del Pezzo base to an involution $\sigma$ on the threefold $\mathcal{M}_{n}$. In fact, by appropriately defining $\sigma$ the action on the $(-1)$ curves of $\mathcal{B}_{n}$ lifts to an action of their $\pi^{*}$ pull-backs. The fixed divisors wrapped by the O7-planes can then be determined using Table 6 . Determining the number of O3-planes in the full set-up also depends on the precise form of the involution on $M_{n}$. In particular, it is not generally the case that each isolated fix-point in the base lifts to a single fix-point in $M_{n}$. Let us consider the case where the torus fibre over the fix-point is smooth, which will turn out to be the case in our explicit examples. In this simple situation, we have to distinguish three cases. Firstly, the fibre over the isolated fix-point can be fixed itself. This extension of the involution should be omitted, since this would imply the presence of O5-planes and violate the condition $\sigma^{*} \Omega=-\Omega$. Secondly, the involution can act as shift on the torus fibre and hence have no fix-points in $M_{n}$. Thirdly, $\sigma$ can act as the inversion of the torus fibre. Such an involution has 4 fix-points, one on the zero section and three on the tri-section

$$
D_{T}=3 \mathcal{B}_{n}-3 \pi^{*}(K),
$$

where $K$ is the canonical class of the del Pezzo base. To define viable involutions on $M_{n}$ one needs to extend this analysis to the singular Kodaira fibres.

\section{Del Pezzo transitions of $\mathbb{P}_{1,1,1,6,9}[18]$}

An alternative way to construct the threefolds $M_{n}$ is to perform del Pezzo transitions starting with the degree 18 hypersurface in weighted projective space $\mathbb{P}_{1,1,1,6,9}$. This Calabi-Yau manifold $M_{0}=\mathbb{P}_{1,1,1,6,9}[18]$ is an elliptic $\mathbb{P}_{1,2,3}[6]$ fibration over the base $\mathcal{B}=\mathbb{P}_{2}$ and has $h^{1,1}=2$. In order to perform the del Pezzo transition $M_{0} \rightarrow M_{1}$ one generates a $\mathrm{dP}_{8}$ singularity by fixing 29 complex structure deformations [71, 41]. This singularity is then resolved by blowing up a del Pezzo surface $\mathrm{dP}_{8}$. Clearly, in this process the Hodge numbers precisely change as required in eq. (800). This process can be repeated to obtain the manifolds $M_{n}$. However, it is important to note that the manifold constructed via the elliptic fibration only coincide with the one obtained by del Pezzo transitions after performing appropriate flop transitions. In order to obtain del Pezzo surfaces $\mathrm{dP}_{8}$ out of the $\mathrm{dP}_{9}$ surfaces of the elliptic fibred phase, one has to perform a flop transition for the $\mathbb{P}^{1}$ intersecting the base. 


\subsection{Orientifold of An Elliptic Fibration Over $\mathrm{dP}_{2}$}

The first Calabi-Yau threefold which we investigate in detail is the manifold $M_{2}$ which corresponds to the elliptic fibration over $\mathrm{dP}_{2}$. The geometry of $M_{2}$ and its topological phases has been studied from a different point of view in [72]. Note that, using eqns. (80) and (79), one finds the Hodge numbers $h^{1,1}\left(M_{2}\right)=4, h^{2,1}\left(M_{2}\right)=214$ and the Euler number $\chi\left(M_{2}\right)=-420$. It will be important that the manifold $M_{2}$ has actually 5 distinct topological phases which are connected by flop transitions. They correspond to the five triangulations of the toric $\mathrm{dP}_{2}$ base. $\mathrm{dP}_{2}$ can be represented torically by the points $(1,0),(0,1),(-1,0),(0,-1),(-1,-1)$ in two dimensions. The five triangulations of this polyhedron are depicted in Figure 1 .
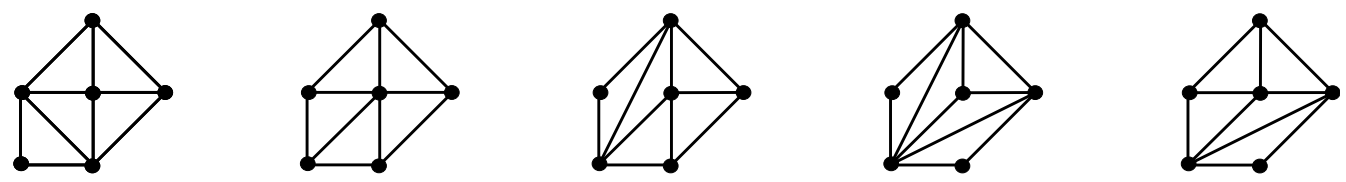

Figure 1: The five different triangulations of the toric $\mathrm{dP}_{2}$ base.

To study the Calabi-Yau space $M_{2}$ in detail, we will also invoke the methods of toric geometry. Note that $\mathbb{P}_{1,1,1,6,9}$ is described by the six points $v_{1}^{*}=(1,0,0,0)$, $v_{2}^{*}=(0,1,0,0), v_{3}^{*}=(0,0,1,0), v_{4}^{*}=(0,0,0,1), v_{5}^{*}=(-9,-6,-1,-1), v_{6}^{*}=$ $(-3,-2,0,0)$. Each of these points corresponds to a divisor $D_{i}$ and the hypersurface $\mathbb{P}_{1,1,1,6,9}[18]$ is defined to admit the anti-canonical class $-\sum_{i=1}^{6} D_{i}$. To obtain the manifold $M_{2}$ one introduces two blowing-up divisors $D_{7}, D_{8}$ corresponding to the points $v_{7}^{*}=(-6,-4,-1,0)$ and $v_{8}^{*}=(-6,-4,0,-1)$. The compact hypersurface with anti-canonical class $-\sum_{i=1}^{8} D_{i}$ is the manifold $M_{2}$. It admits five triangulations just as the $\mathrm{dP}_{2}$ base itself. In the following we will investigate two of them in more detail. The corresponding Calabi-Yau space will be denoted by $M_{2}^{\left(\mathrm{dP}_{9}\right)^{2}}$ and $M_{2}^{\left(\mathrm{dP}_{8}\right)^{2}}$. Here we indicate the type of the divisors $D_{7}, D_{8}$ as we will check below 20 .

\footnotetext{
${ }^{20}$ We are grateful to Albrecht Klemm for help with the programs to perform the toric computations. The analysis of the divisors and their intersection ring was carried out by using the Maple code Schubert.
} 


\section{The geometry of $\mathrm{M}_{2}^{(\mathrm{dP} 9)^{2}}$}

Let us first discuss the Calabi-Yau manifold $M_{2}^{\left(\mathrm{dP}_{9}\right)^{2}}$. The data of the associated linear sigma model is the following. We have eight complex coordinates $x_{i}$. The divisors $D_{i}$ are defined by the constraints $x_{i}=0$. In addition there are four $U(1)$ symmetries. The corresponding charges are shown in (83). Note that we have chosen the charge vectors to correspond to the Mori cone generators for this triangulation.

\begin{tabular}{|c|c|c|c|c|c|c|c|c||c|}
\hline & $x_{1}$ & $x_{2}$ & $x_{3}$ & $x_{4}$ & $x_{5}$ & $x_{6}$ & $x_{7}$ & $x_{8}$ & $p$ \\
\hline \hline$\ell^{(1)}$ & 3 & 2 & 0 & 0 & 0 & 1 & 0 & 0 & 6 \\
$\ell^{(2)}$ & 0 & 0 & 1 & 0 & 1 & -1 & 0 & -1 & 0 \\
$\ell^{(3)}$ & 0 & 0 & 0 & 1 & 1 & -1 & -1 & 0 & 0 \\
$\ell^{(4)}$ & 0 & 0 & 0 & 0 & -1 & -1 & 1 & 1 & 0 \\
\hline
\end{tabular}

The Mori cone for this triangulation is generated by four holomorphic curves $C^{a}$ which intersect the divisors $D_{i}$ as $D_{i} \cdot C^{a}=\ell_{i}^{(a)}$. Hence, the $\ell^{(a)}$ are the coordinates of the $C^{a}$ in the two-cycle basis dual to $D_{i}$. Since there are as much Mori generators as Kähler parameters $h^{1,1}=4$, this Mori cone is simplicial. Using the Mori generators it is also straightforward to determine a basis $K_{i}$ of four-cycles generating the Kähler cone. Expanding the Kähler form as $J=r^{i}\left[K_{i}\right]$ the condition that all physical volumes are positive translates into

$$
\int_{C^{a}} J=r^{i} C^{a} \cdot K_{i}>0
$$

This requires $r^{i}>0$ for

$$
\begin{array}{ll}
K_{1}=3 D_{5}+D_{6}+2 D_{7}+2 D_{8}, & K_{2}=D_{5}+D_{7} \\
K_{3}=D_{5}+D_{8}, & K_{4}=D_{5}+D_{7}+D_{8} .
\end{array}
$$

In this basis all triple intersections are positive, ensuring positivity of the divisor volumes and the total volume of $Y$. However, for our applications it is more useful to display the triple intersection numbers in the basis $\left\{D_{5}, D_{6}, D_{7}, D_{8}\right\}$ as

$$
I_{3}=D_{6}\left(7 D_{6}^{2}-D_{5}^{2}-D_{7}^{2}-D_{8}^{2}-D_{5} D_{6}-D_{6} D_{7}-D_{6} D_{8}+D_{5} D_{7}+D_{5} D_{8}\right) .
$$

Not surprisingly, there are both negative and positive intersections in this basis.

To determine the geometry of the different divisors $D_{i}$ we now compute the Euler characteristic $\chi=\int_{D} c_{2}\left(T_{D}\right)$, as well as $\int_{D} c_{1}^{2}\left(T_{D}\right)$ for each divisor in $M_{2}^{\left(\mathrm{dP}_{9}\right)^{2}}$. We 
exemplify this for the divisor $D_{8}$ and restrict the intersection form (86) to this surface

$$
I_{D_{8}}=-D_{6}^{2}-D_{6} D_{8}+D_{5} D_{6}
$$

Using this intersection form one computes

$$
\chi\left(D_{8}\right)=12, \quad \int_{D_{8}} c_{1}^{2}\left(T_{D_{8}}\right)=0
$$

From this we conclude that $D_{8}=\pi^{*}\left(E_{2}\right)$ is a $\mathrm{dP}_{9}$ surface. Analogously, we proceed for the remaining divisors $D_{i}$. We identify

$$
\begin{array}{llll}
D_{3}=\pi^{*}\left(l-E_{2}\right) & K 3, & D_{4}=\pi^{*}\left(l-E_{1}\right) & K 3, \\
D_{5}=\pi^{*}\left(l-E_{1}-E_{2}\right) & \mathrm{dP}_{9}, & D_{6}=\mathcal{B} & \mathrm{dP}_{2}, \\
D_{7}=\pi^{*}\left(E_{1}\right) & \mathrm{dP}_{9}, & D_{8}=\pi^{*}\left(E_{2}\right) & \mathrm{dP}_{9} .
\end{array}
$$

Let us note that indeed the exceptional divisors $D_{7}$ and $D_{8}$ are $\mathrm{dP}_{9}$ surfaces which justifies our notation $M_{2}^{\left(\mathrm{dP}_{9}\right)^{2}}$. Finally, using (83) the divisor $D_{1}$ can be identified with

$$
D_{1}=3 \mathcal{B}+9 \pi^{*}\left(l-E_{1}-E_{2}\right)+6 \pi^{*}\left(E_{1}\right)+6 \pi^{*}\left(E_{2}\right)=D_{T},
$$

that is, the tri-section $D_{T}$ defined in (82) of the elliptic fibration over $\mathcal{B}=\mathrm{dP}_{2}$. With these identifications one can check that the triple intersection form (86) is indeed the one generated by $\left\{\mathcal{B}, \pi^{*}\left(E_{i}\right), \pi^{*}\left(l-E_{1}-E_{2}\right)\right\}$. All terms in (86) contain the base $D_{6}$ and the expression in the brackets corresponds to the intersection form of $E_{i}$ and $l-E_{1}-E_{2}$ as well as the self-intersection 7 of the anti-canonical class on $\mathrm{dP}_{2}$.

Let us now specify an orientifold projection $\Omega_{p} \sigma(-1)^{F_{L}}$. As a simple example consider the involution

$$
\sigma: x_{3} \rightarrow-x_{3}
$$

and analyse the fixed point set. In order to do that we first note that the coordinates obey some scaling relations dictated by the $U(1)$ weights $\ell^{(k)}$ displayed in (83). This implies that in order to determine the fix-point set of (91), the coordinates $x_{i}$ need only to agree up to scaling such that

$$
x_{j}= \pm \prod_{k=1}^{4} \lambda_{k}^{\ell_{j}^{(k)}} x_{j},
$$

where $\lambda_{k} \in \mathbb{C}^{*}$, and the minus sign should be used for $x_{3}$ while the plus sign holds for all other coordinates. The value of the complex scalars $\lambda_{k}$ is restricted by the 
Stanley-Reisner ideal of the toric ambient space. More precisely, this ideal contains the information which coordinates $x_{i}$ are not allowed to vanish simultaneously. For the case at hand it reads

$$
S R=\left\{x_{3} x_{5}, x_{3} x_{7}, x_{4} x_{5}, x_{4} x_{8}, x_{7} x_{8}, x_{1} x_{2} x_{6}\right\} .
$$

For example, since $x_{1} x_{5}$ is in the Stanley-Reisner ideal, the subspace $x_{1}=x_{5}=0$ is not in the toric variety. Combining these conditions with the scalings eq. (92) fixes the $\lambda_{i}$ to specific values and allows us to determine the fix-point locu 21 .

Let us apply this strategy explicitly to our example. The divisors $D_{3}=\left\{x_{3}=0\right\}$ and $D_{7}=\left\{x_{7}=0\right\}$ are fixed under $\sigma$ consistent with the scalings (83). As mentioned, $D_{3}$ is a $K 3$ surface with $\chi=24$ and $D_{7}$ is a $\mathrm{dP}_{9}$ with $\chi=12$. However, this is not the end of the story, as there exist also fixed points which give the location of $O 3$-planes. Using the projective identifications, we first get the two candidate fixed points

$$
p_{1}=\left\{x_{4}=x_{5}=x_{6}=0\right\}, \quad p_{2}=\left\{x_{5}=x_{6}=x_{8}=0\right\},
$$

where however, the first one $p_{1}$ is part of the Stanley-Reisner ideal and therefore discarded. In addition there exist two fixed loci

$$
p_{3}=\left\{x_{1}=x_{4}=x_{5}=0\right\}, \quad p_{4}=\left\{x_{1}=x_{5}=x_{8}=0\right\},
$$

where again the first is discarded and the second actually consists of 3 fixed points, which is essentially the space $\mathbb{P}_{1,2}[6]$. Note that this is precisely the situation discussed in Subsection 4.2. From eq. (94) one infers that the involution eq. (91) admits one isolated fix-point in the base $D_{6} . \sigma$ acts on the smooth torus fibre over this point as inversion, such that three fix-points eq. (95) arise in the tri-section $D_{1}$.

To summarise, the fixed points locus consists of two non-intersecting $O 7$ planes and four $O 3$ planes. Therefore, the right-hand side of the D7-brane tadpole cancellation condition (13) reads

$$
8\left[D_{O 7}\right]=8 \pi^{*}\left(l-E_{2}\right)+8 \pi^{*}\left(E_{1}\right)
$$

and the right-hand side of the $D 3$ brane tadpole condition (17) takes the form

$$
\frac{\chi(K 3)+\chi\left(\mathrm{dP}_{9}\right)}{6}+\frac{N_{O 3}}{2}=8 .
$$

\footnotetext{
${ }^{21}$ Note that in general, the determination of the fix-point set in the Calabi-Yau hypersurface can be more tricky. This is due to fact that it will in general be non-generic hypersurface to admit the involution $\sigma$. In our examples, this will not introduce any new subtleties.
} 
Note that indeed we get a non-negative integer. In order to cancel the $D 7$ brane tadpole we can only introduce branes wrapping entirely the fibre. Candidates are of course $D_{3}$ and $D_{7}$ which are point-wise invariant under the orientifold projection and therefore belong to class 3.) introduced in Subsection 2.1, A natural candidate of class 2.) is $D_{5}=\pi^{*}\left(l-E_{1}-E_{2}\right)$. This $\mathrm{dP}_{9}$ is not point-wise invariant. In fact, using that $D_{5}$ intersects the O7-plane in a genus 1 curve and that all four O3 planes are inside $D_{5}$ one uses the Lefschetz fix-point formula [73] to compute for $D_{5}$ that $b_{+}^{2}=6$ and $b_{-}^{2}=4$. This involution indeed corresponds to a viable involution of $\mathrm{dP}_{9}$.

Let us also consider the involution $x_{1} \rightarrow-x_{1}$, which is nothing else than the reflection of the torus fibre. The fixed point locus can be determined as $D_{O 7}=$ $D_{1} \cup D_{6}$, such that the right hand side of (13) reads

$$
8\left[D_{O 7}\right]=32 \mathcal{B}+24 \pi^{*} c_{1}(\mathcal{B})
$$

and no fixed points. For the Euler characteristics we find $\chi\left(D_{1}\right)=435$ and $\chi\left(D_{6}\right)=$ $\chi\left(\mathrm{dP}_{2}\right)=5$, such that the right hand side of (17) takes the form

$$
\frac{\chi\left(D_{O 7}\right)}{6}+\frac{N_{O 3}}{2}=\frac{220}{3} .
$$

Finally, let us determine how this involution acts on $\pi^{*}\left(E_{1}\right)=\mathrm{dP}_{9}$. The divisor $\mathcal{B}$ intersects $\pi^{*}\left(E_{1}\right)$ of course over a sphere and from $\chi\left(D_{1} \cap D_{7}\right)=D_{1} \cdot D_{7} \cdot\left(-D_{1}-D_{7}\right)=$ -6 we conclude that the Euler characteristic of the fix-point set in $\mathrm{dP}_{9}$ is $\chi=-4$. This implies $b_{+}^{2}=2$ and $b_{-}^{2}=8$ and corresponds to the blow-up of the Bertini involution $\left(\mathcal{B}_{8}, \sigma_{\mathrm{B}}\right)$ of Table 6 at one invariant point.

\section{The geometry of $\mathrm{M}_{2}^{\left(\mathrm{dP}_{8}\right)^{2}}$}

Let us now consider the triangulation which corresponds to a Calabi-Yau manifold with two exceptional $\mathrm{dP}_{8}$ divisors $D_{7}$ and $D_{8}$. Again we specify the data for the linear sigma-model such that the charge vectors correspond to the Mori cone generators for this triangulation.

\begin{tabular}{|c|c|c|c|c|c|c|c|c||c|}
\hline & $x_{1}$ & $x_{2}$ & $x_{3}$ & $x_{4}$ & $x_{5}$ & $x_{6}$ & $x_{7}$ & $x_{8}$ & $p$ \\
\hline \hline$\ell^{(1)}$ & 3 & 2 & 0 & 1 & 1 & 0 & -1 & 0 & 6 \\
$\ell^{(2)}$ & 3 & 2 & 1 & 0 & 1 & 0 & 0 & -1 & 0 \\
$\ell^{(3)}$ & 0 & 0 & 0 & -1 & -1 & 1 & 1 & 0 & 0 \\
$\ell^{(4)}$ & 0 & 0 & 1 & 1 & 1 & -3 & 0 & 0 & 0 \\
$\ell^{(5)}$ & 0 & 0 & -1 & 0 & -1 & 1 & 0 & 1 & 0 \\
\hline
\end{tabular}


Note that the Mori cone eq. (100) is generated by five holomorphic curves $C^{a}$ in this triangulation. Since this is more than the $h^{1,1}=4$ Kähler deformations the Mori cone is non-simplicial. Also for this case we have to determine the dual Kähler cone spanned by four-cycles. By definition the Kähler cone is generated by four-cycles $D$ which satisfy $D \cdot C^{a} \geq 0$. For our purposes it will be convenient to determine the Kähler cone in coordinates $r^{i}$. We therefore chose to discard one of the 5 Mori vectors (100), namely, the last one $\ell^{(5)}$. As in the $M_{2}^{\left(\mathrm{dP}_{9}\right)^{2}}$ phase it is then straightforward to determine the Kähler cone generators

$$
\begin{aligned}
& K_{1}=3 D_{5}+D_{6}+2 D_{7}+3 D_{8}, \quad K_{2}=-D_{8}, \\
& K_{3}=3 D_{5}+D_{6}+3 D_{7}+3 D_{8}, \quad K_{4}=D_{5}+D_{7}+D_{8} \text {. }
\end{aligned}
$$

Note that for $J=r^{i}\left[K_{i}\right]$, the positivity of the curves $C^{i}, i=1, \ldots, 4$ is ensured when $r^{i}>0$. In order that the last curve is positive we have to additionally impose $\int_{C^{5}} J=r^{i} K_{i} \cdot C^{5}=r^{1}-r^{2}+r^{3}>0$. Clearly, a similar analysis can be carried out when discarding one of the other $\ell^{(a)}$ which allows to define coordinates for the complete non-simplicial Kähler cone.

For convenience we again display the triple intersection numbers for $M_{2}^{\left(\mathrm{dP}_{8}\right)^{2}}$ in the basis $\left\{D_{5}, D_{6}, D_{7}, D_{8}\right\}$,

$$
I_{3}=9 D_{6}^{3}+D_{7}^{3}+D_{8}^{3}+D_{5}\left(D_{5} D_{6}+D_{5} D_{7}+D_{5} D_{8}-2 D_{5}^{2}-3 D_{6}^{2}-D_{7}^{2}-D_{8}^{2}\right) .
$$

In particular, for the intersection form on the surface $D_{8}$ we get

$$
I_{D_{8}}=D_{5}^{2}+D_{8}^{2}-D_{5} D_{8}
$$

so that

$$
\chi\left(D_{8}\right)=11, \quad \int_{D_{8}} c_{1}^{2}\left(T_{D_{8}}\right)=1
$$

which we identify with the correct values for $\mathrm{dP}_{8}$. For $D_{7}$ we find the same result. Comparing this result with (88) we note that in this cone of the complexified Kähler moduli space, one $\mathbb{P}^{1}$ in each $\pi^{*}\left(E_{i}\right)$ of $M_{2}^{\left(\mathrm{dP}_{9}\right)^{2}}$ has been flopped away so that $\mathrm{dP}_{9} \rightarrow$ $\mathrm{dP}_{8}$. Indeed the two exceptional divisors $E_{i}$ of the $\mathrm{dP}_{2}$ base in $M_{2}^{\left(\mathrm{dP}_{9}\right)^{2}}$ are absent in $M_{2}^{\left(\mathrm{dP}_{8}\right)^{2}}$ since $\chi\left(D_{6}\right)=3$, and $\int c_{2}\left(T_{D_{6}}\right)=9$.

Having performed the flop transitions, the exceptional $\mathbb{P}^{1} \mathrm{~s}$ have to reappear in other divisors. In fact, we compute

$$
\chi\left(D_{3}\right)=25, \quad \chi\left(D_{5}\right)=14, \quad \int c_{1}^{2}\left(T_{D_{3}}\right)=-1, \quad \int c_{1}^{2}\left(T_{D_{5}}\right)=-2 .
$$


Again, the divisor $D_{4}$ has the same topology as $D_{3}$. This implies that the divisor $D_{3}, D_{4}$ corresponding to the pull-back divisors $\pi^{*}\left(l-E_{i}\right)$ in $M_{2}^{\left(\mathrm{dP}_{9}\right)^{2}}$ now contain each one additional $\mathbb{P}^{1}$. The divisor $D_{5}$ corresponding to $\pi^{*}\left(l-E_{1}-E_{2}\right)$ contains two additional $\mathbb{P}^{1} \mathrm{~s}$.

Let us now investigate the involutions on $M_{2}^{\left(\mathrm{dP}_{8}\right)^{2}}$. A simple involution exchanging the two $\mathrm{dP}_{8}$ surfaces has been employed in [74]. However, since these two del Pezzo surfaces do not intersect, this involution will not be useful in constructing GUT models. We therefore consider again the involution $\sigma: x_{3} \rightarrow-x_{3}$, which still has the non-intersecting $O 7$ planes $D_{3}$ and $D_{7}$. There exist 4 fixed points so that the tadpole contribution is

$$
\frac{\chi\left(D_{O 7}\right)}{6}+\frac{N_{O 3}}{2}=8
$$

This condition is identical to the condition eq. (97) on $M_{2}^{\left(\mathrm{dP}_{9}\right)^{2}}$ since the topology change of the two $\mathrm{O} 7$ divisors precisely cancels. One checks that 3 fix-points are located on the del Pezzo 8 defined by $x_{8}=0$ which intersects the orientifold locus $D_{3}$ on a genus 1 curve. This implies using Table 6 that $b_{+}^{2}=5, b_{-}^{2}=4$ for the del Pezzo 8.

The involution defined by the reflection $x_{1} \rightarrow-x_{1}$, again has the fixed point divisors $D_{O 7}=D_{1} \cup D_{6}$. For the Euler characteristics we find $\chi\left(D_{1}\right)=435$ and $\chi\left(D_{6}\right)=3$. However, this time the involution also has the two fixed points $p_{1}=$ $\left\{x_{4}=x_{5}=x_{7}=0\right\}$ and $p_{2}=\left\{x_{3}=x_{5}=x_{8}=0\right\}$. For the D3-tadpole contribution we therefore obtain

$$
\frac{\chi\left(D_{O 7}\right)}{6}+\frac{N_{O 3}}{2}=\frac{222}{3} .
$$

Finally, let us determine how this involution acts on $D_{7}=\mathrm{dP}_{8}$. The base $\mathcal{B}=D_{6}$ does not intersect $D_{7}$ after the flop transition, while $D_{1}$ intersects $D_{7}$ over curve with $\chi=-6$. Moreover, only the fixed point $p_{1}$ lies on $D_{7}$, while $p_{2}$ lies on $D_{8}$. Therefore, the Euler-characteristic of the fixed point set in $D_{7}$ is $\chi=-5$ and we obtain $b_{2}^{+}=1, b_{2}^{-}=8$. Comparing this result with Table 6 we conclude that the involution $x_{1} \rightarrow-x_{1}$ acts on the $\mathrm{dP}_{8}$ as the Bertini involution $\left(\mathcal{B}_{8}, \sigma_{\mathrm{B}}\right)$.

\subsection{Orientifold of An Elliptic Fibration Over $\mathrm{dP}_{3}$}

We can repeat the procedure just described also for the del Pezzo base $\mathrm{dP}_{3}$. This remains to be rather simple, since this del Pezzo is still toric and represented by the 


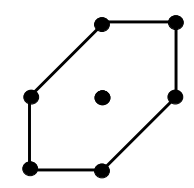

Figure 2: The points of the polyhedron of the $\mathrm{dP}_{3}$ base.

points $(1,0),(0,1),(-1,0),(0,-1),(-1,-1)$ and $(1,1)$ as shown in Figure 2. In this case there are in fact 18 triangulations of the $\mathrm{dP}_{3}$ base. The toric ambient space for the corresponding Calabi-Yau hypersurface $M_{3}$ is obtained by adding the point $v_{9}^{*}=(3,2,1,1)$ to the polyhedron of $M_{2}$ above. This polyhedron has 18 triangulations which yield different phases of the Calabi-Yau hypersurface $M_{3}$. As determined by eqns. (80) and (79), each $M_{3}$ has the topological data $h^{1,1}\left(M_{3}\right)=5, h^{2,1}\left(M_{3}\right)=185$, and $\chi\left(M_{3}\right)=-360$. In the following we discuss one out of the 18 phases in more detail. Namely, the Weierstraß phases $M_{3}^{\left(\mathrm{dP}_{9}\right)^{3}}$ where the divisors $D_{7}, D_{8}, D_{9}$ are $\mathrm{dP}_{9}$ surfaces. A second phase, $M_{3}^{\left(\mathrm{dP}_{8}\right)^{3}}$ will be of importance in Subsection 4.6 where we discuss the issue of moduli stabilisation for compactifications on $M_{n}$. On $M_{3}$ we will also be able to introduce interesting orientifold projections with a non-trivial split $h^{1,1}=h_{+}^{1,1}+h_{-}^{1,1}$.

\section{The geometry of $\mathrm{M}_{3}^{\left(\mathrm{dP}_{9}\right)^{3}}$}

Let us discuss the Weierstraß phase where all $(-1)$-curves lead to $\mathrm{dP}_{9}$ surfaces. Using Table 4 we infer that $\mathrm{dP}_{3}$ has six $(-1)$-curves which yield six $\mathrm{dP}_{9}$ surfaces. The Mori cone associated to this phase is shown in eq. (108).

\begin{tabular}{|c|c|c|c|c|c|c|c|c|c||c|}
\hline & $x_{1}$ & $x_{2}$ & $x_{3}$ & $x_{4}$ & $x_{5}$ & $x_{6}$ & $x_{7}$ & $x_{8}$ & $x_{9}$ & $p$ \\
\hline \hline$\ell^{(1)}$ & 3 & 2 & 0 & 0 & 0 & 1 & 0 & 0 & 0 & 6 \\
$\ell^{(2)}$ & 0 & 0 & 0 & 1 & 1 & -1 & -1 & 0 & 0 & 0 \\
$\ell^{(3)}$ & 0 & 0 & 0 & 0 & -1 & -1 & 1 & 1 & 0 & 0 \\
$\ell^{(4)}$ & 0 & 0 & 1 & 1 & 0 & -1 & 0 & 0 & -1 & 0 \\
$\ell^{(5)}$ & 0 & 0 & 0 & -1 & 0 & -1 & 1 & 0 & 1 & 0 \\
$\ell^{(6)}$ & 0 & 0 & 1 & 0 & 1 & -1 & 0 & -1 & 0 & 0 \\
$\ell^{(7)}$ & 0 & 0 & -1 & 0 & 0 & -1 & 0 & 1 & 1 & 0 \\
\hline
\end{tabular}

Using the data for the Mori cone, it is straightforward to evaluate the associated Kähler cone. However, we again display the triple intersection numbers in the basis 


$$
\begin{aligned}
& D_{6}, \ldots, D_{9} \\
& I_{3}=D_{6}\left(6 D_{6}^{2}-D_{5}^{2}-D_{7}^{2}-D_{8}^{2}-D_{9}^{2}-D_{6}\left(D_{5}+D_{7}+D_{8}+D_{9}\right)+D_{5} D_{8}+D_{7} D_{5}\right) .
\end{aligned}
$$

In a similar spirit as in the previous section we can also check that the divisors

$$
\begin{array}{llll}
D_{3} & =\pi^{*}\left(l-E_{1}-E_{3}\right), & D_{4}=\pi^{*}\left(l-E_{2}-E_{3}\right), & D_{5}=\pi^{*}\left(l-E_{1}-E_{2}\right), \\
D_{7}=\pi^{*}\left(E_{2}\right), & D_{8}=\pi^{*}\left(E_{1}\right), & D_{9}=\pi^{*}\left(E_{3}\right) .
\end{array}
$$

are the six $\mathrm{dP}_{9}$ surfaces, while the $\mathrm{dP}_{3}$ base is the divisor $D_{6}$.

Let us now turn to the definition of the involution on $M_{3}^{\left(\mathrm{dP}_{9}\right)^{3}}$. Using Table 6, we find four candidate involutions on the $\mathrm{dP}_{3}$ base. Our prime focus will be on the exchange involution $\left(\mathcal{B}_{3}, \sigma_{3}\right)$, which admits the rational curve $2 l-E_{1}-E_{2}$ as fix-point divisor. Lifted to the elliptically fibred threefold, this involution descends to

$$
\sigma: \quad x_{7} \leftrightarrow x_{3}, \quad x_{8} \leftrightarrow x_{4}, \quad x_{9} \leftrightarrow x_{5}
$$

We can thus evaluate the action of $\sigma^{*}$ on the cohomology $H^{2}\left(M_{3}\right)$ spanned by $\left[D_{5}, D_{6}, D_{7}, D_{8}, D_{9}\right]$ to show that

$$
h_{+}^{1,1}=3, \quad h_{-}^{1,1}=2 .
$$

This implies that by using (1) this orientifold compactification will admit three Kähler moduli $T_{I}$ and two B-field moduli $G^{i}$.

Again, we can determine the fixpoint set of $\sigma$ using toric geometry. Taking into account the scaling relations (108) and the corresponding constraints from the Stanley-Reisner ideal, one finds the fix-point divisor

$$
x_{5} x_{8} x_{3} x_{9}-x_{4} x_{9} x_{5} x_{7}=0,
$$

and no isolated fix-points meeting the hypersurface. The isolated fix-points and hence O3-planes can also be directly inferred from the fact that there are no fixpoints on the $\mathrm{dP}_{3}$ base for this involution. In accord with the general arguments presented in Subsection 4.2, the locus eq. (113) corresponds to an O7-plane on the divisor class $D_{O 7}=D_{4}+D_{9}+D_{5}+D_{7}=\pi^{*}\left(2 l-E_{1}-E_{2}\right)$ and induces a tadpole

$$
8\left[D_{O 7}\right]=8 \pi^{*}\left(2 l-E_{1}-E_{2}\right) .
$$

For this O7-plane one computes $\chi\left(D_{O 7}\right)=48$, such that the induced D3-tadpole is $\chi\left(D_{O 7}\right) / 6=8$. 


\subsection{The Swiss-Cheese Property}

Though we will deliver some more comments in Section 8, in this paper we do not yet intend to combine the GUT model search with a complete analysis of moduli stabilisation. However, we would like to point out that some of the manifolds discussed so far provide new examples of so-called swiss-cheese type Calabi-Yau manifolds. Here we understand this term in the strong sense that the volume $\mathcal{V}$ of the Calabi-Yau can be expressed a: 22

$$
\mathcal{V}=\frac{\sqrt{8}}{6}\left[\frac{1}{\sqrt{3^{5}}}\left(\tau_{0}\right)^{\frac{3}{2}}-\frac{1}{3}\left(\tau_{\mathcal{B}}\right)^{\frac{3}{2}}-\sum_{i=1}^{h^{1,1}-2}\left(\tau_{i}\right)^{\frac{3}{2}}\right],
$$

where the numerical coefficients are chosen for later convenience and $\tau_{B}, \tau_{i}$ are volumes of a basis of four-cycles $\Gamma_{0}, \Gamma_{\mathcal{B}}, \Gamma_{i}$ given by

$$
\tau_{0}=\frac{1}{2} \int_{\Gamma_{B}} J \wedge J, \quad \tau_{\mathcal{B}}=\frac{1}{2} \int_{\Gamma_{\mathcal{B}}} J \wedge J, \quad \tau_{i}=\frac{1}{2} \int_{\Gamma_{i}} J \wedge J .
$$

Our aim is to show that for the manifolds $M_{n}^{\left(\mathrm{dP}_{8}\right)^{n}}$ one can always find such a basis $\Gamma_{0}, \Gamma_{\mathcal{B}}, \Gamma_{i}$ such that $\mathcal{V}$ is of the form (115).

\section{Swiss-Cheese property of $\mathrm{M}_{2}^{\left(\mathrm{dP}_{8}\right)^{2}}$ and $\mathrm{M}_{3}^{\left(\mathrm{dP}_{8}\right)^{3}}$}

We first consider the manifold $M_{2}^{\left(\mathrm{dP}_{8}\right)^{2}}$. Recall that this Calabi-Yau is connected via a flop transition to the corresponding Weierstraß model, which is an elliptic fibration over the $\mathrm{dP}_{2}$ base. In fact, the two exceptional two cycles $E_{1,2}$ in the base $\mathrm{dP}_{2}$ have been flopped away. We will use the same notation as in Sections 4.3 and 4.4. The triple intersection form on $M_{2}^{\left(\mathrm{dP}_{8}\right)^{2}}$ in the basis $D_{i}$ was given in eq. (102). Expanding the Kähler form as $J=r_{5} D_{5}+r_{6} D_{6}+r_{7} D_{7}+r_{8} D_{8}$ and defining

$$
\Gamma_{\mathcal{B}}=D_{6}, \quad \Gamma_{1}=D_{7}, \quad \Gamma_{2}=D_{8},
$$

we compute the $\tau_{i}$ in (116) as

$$
\tau_{\mathcal{B}}=\frac{1}{2}\left(r_{5}-3 r_{6}\right)^{2}, \quad \tau_{1}=\frac{1}{2}\left(r_{5}-r_{7}\right)^{2}, \quad \tau_{2}=\frac{1}{2}\left(r_{5}-r_{8}\right)^{2} .
$$

\footnotetext{
${ }^{22}$ It has been shown [66] that a much weaker condition is already sufficient to make the LARGE volume scenario work.
} 
Now, let us define the following divisor, which obviously is related to the former tri-section $D_{1}$ in the Weierstraß phase

$$
\Gamma_{0}=D_{1}+3 D_{7}+3 D_{8}=3\left(3 D_{5}+D_{6}+3 D_{7}+3 D_{8}\right) .
$$

The volume of this divisor is given by $\tau_{0}=\frac{3}{2} r_{5}^{2}$. We have found four divisors whose volumes can be written as perfect squares and it is now a simple calculation to show that the total volume of the Calabi-Yau can be written as in (115), showing the swiss cheese structure. Note that indeed the three small cycles are of the type $\Gamma_{\mathcal{B}}=\mathbb{P}_{2}$, $\Gamma_{1,2}=\mathrm{dP}_{8}$ and therefore all are shrinkable to a point.

Along the same lines also the swiss cheese structure of the Calabi-Yau $M_{3}^{\left(\mathrm{dP}_{8}\right)^{3}}$ can be shown. This manifold is related to the Weierstraß model over $\mathrm{dP}_{3}$. For completeness, let us list the relevant data. The intersection form reads

$$
\begin{gathered}
I_{3}=-2 D_{5}^{3}+9 D_{6}^{3}+D_{7}^{3}+D_{8}^{3}+D_{9}^{3}+D_{5}^{2} D_{6}-3 D_{5} D_{6}^{2}+D_{5}^{2} D_{7}-D_{5} D_{7}^{2} \\
\quad+D_{5}^{2} D_{8}-D_{5} D_{8}^{2} .
\end{gathered}
$$

For the volumes of the del Pezzo type divisors $\Gamma_{\mathcal{B}}=D_{6}, \Gamma_{i}=D_{i+6}$ we get

$$
\tau_{\mathcal{B}}=\frac{1}{2}\left(r_{5}-3 r_{6}\right)^{2}, \quad \tau_{1}=\frac{1}{2}\left(r_{5}-r_{7}\right)^{2}, \quad \tau_{2}=\frac{1}{2}\left(r_{5}-r_{8}\right)^{2}, \quad \tau_{3}=\frac{1}{2} r_{9}^{2},
$$

and with

$$
\Gamma_{0}=D_{1}+3 D_{7}+3 D_{8}+3 D_{9}=3\left(3 D_{5}+D_{6}+3 D_{7}+3 D_{8}\right) .
$$

and $\tau_{0}=\frac{3}{2} r_{5}^{2}$ the total volume of the Calabi-Yau can again be written as eq. (115).

\section{Proof of Swiss-Cheese property for $\mathrm{M}_{\mathrm{n}}^{(\mathrm{dP})^{\mathrm{n}}}$}

What we have concretely confirmed for the latter two examples of Calabi-Yau threefolds is, in fact, more generally true. Starting with the Weierstraß phase of an elliptic fibration over a $\mathrm{dP}_{n}, n=0, \ldots, 8$ base, the phase related to this one by flopping away all $n \mathbb{P}^{1}$-cycles in the base, is of the swiss-cheese type. To prove this, we show that we can define $n+2$ divisors such that the triple intersection form is diagonal. Before the flop transition we have the pull-back divisors $\pi^{*}\left(E_{i}\right)=\mathrm{dP}_{9}$. After the flop transition, these lose the $\mathbb{P}^{1}$ given by $\mathcal{B} \cap \pi^{*}\left(E_{i}\right)$ and we get $\Gamma_{i}:=\pi^{*}\left(E_{i}\right)_{\text {flop }}=\mathrm{dP}_{8}$ for $i=1, \ldots, n$. Clearly, these divisors satisfy

$$
\Gamma_{i}^{3}=1, \quad \Gamma_{i} \cap \Gamma_{j}=0 \quad \text { for } i \neq j .
$$


The former base $\mathcal{B}=\mathrm{dP}_{n}$ is now just $\mathbb{P}^{2}$ so that we define $\Gamma_{\mathcal{B}}:=\mathcal{B}_{\text {flop }}=\mathbb{P}^{2}$. Since we have flopped away the intersection locus with the $\pi^{*}\left(E_{i}\right)$, we can write

$$
\Gamma_{\mathcal{B}}^{3}=\mathcal{B}_{\text {flop }}^{3}=c_{1}^{2}\left(\mathbb{P}^{2}\right) \mathcal{B}_{\text {flop }}=9, \quad \Gamma_{\mathcal{B}} \cap \Gamma_{i}=0
$$

For the remaining divisor we start with the former tri-section $3\left(\mathcal{B}+3 \pi^{*}(l)-\sum_{i} \pi^{*}\left(E_{i}\right)\right)$ and realise that, after the flop transition, this divisors gains an extra of $3 n \mathbb{P}^{1} \mathrm{~s}$. Therefore, this divisor cannot be diagonal to the ones introduced so far. However, we can define the new divisor

$$
\begin{aligned}
\Gamma_{0} & =3\left(\mathcal{B}+3 \pi^{*}(l)-\sum_{i} \pi^{*}\left(E_{i}\right)\right)_{\text {flop }}+3 \sum_{i} \pi^{*}\left(E_{i}\right)_{\text {flop }} \\
& =3\left(\mathcal{B}_{\text {flop }}+3 \pi^{*}(l)\right)
\end{aligned}
$$

which satisfies

$$
\Gamma_{0}^{3}=243, \quad \Gamma_{0} \cap \Gamma_{i}=0, \quad \Gamma_{0} \cap \Gamma_{\mathcal{B}}=0 .
$$

Therefore, we have found a basis of $(n+2)$ divisors which diagonalise the triple intersection form. Taking into account that except $\Gamma_{0}$ all four-cycles are shrinkable to a point, we expect that inside the Kähler cone, we can write the volume of the Calabi-Yau in the swiss cheese form eq. (115). Apparently, the two former toric Calabi-Yau manifolds are only two specific examples.

For realising the LARGE volume scenario it is not necessary to have a Calabi-Yau having the strong swiss-cheese type property [66] as in (115). Therefore, one can also discuss the case that from the $n$ initial $\pi^{*}\left(E_{i}\right)$ divisors of the type $\mathrm{dP}_{9}$ only $r$ have been flopped to the $\mathrm{dP}_{8}$ phase. Since $\mathrm{dP}_{9}$ is not shrinkable to a point, one does not expect a swiss-cheese type structure for them, but along the same lines as above one can still write the volume as

$$
\mathcal{V}=\mathcal{V}\left(M_{n-r}^{\left(\mathrm{dP}_{9}\right)^{n-r}}\right)-\sum_{i=1}^{r}\left(\tau_{i}\right)^{\frac{3}{2}}
$$

where $M_{n-r}^{\left(\mathrm{dP}_{9}\right)^{n-r}}$ denotes the Weierstraß phase of the elliptic fibration over $d P_{n-r}$.

\subsection{D-Term Conditions For D7-Branes on Del Pezzo Sur- faces}

In this section we have introduced a specific class of manifolds which admit shrinkable del Pezzo surfaces as divisors. In the following we like to address the question 
whether we can wrap D7-branes on these surfaces and stabilise their volume at sizes significantly larger than the string scale by demanding a vanishing D-term, eq. (39).

Let us denote the del Pezzo surface with a wrapped D7-brane by $\left(D_{d P}, L_{d P}\right)$ and its orientifold image by $\left(D_{d P}^{\prime}, L_{d P}^{\prime}\right)$. In the following discussion it is crucial to again distinguish the three cases defined at the beginning of Subsection 2.1, page 11. We will focus on the cases 2 and 3 where $D_{d P}$ and $D_{d P}^{\prime}$ are in the same homology class. This implies that $D_{d P}$ cannot support $B_{-}$moduli and the D-term arises entirely from the gauging $X_{a I}$ given in (37). The precise form of $X_{a I}$ depends on the choice of four-cycles to define the coordinates $T_{I}$. Let us focus on the swiss-cheese examples of Subsection 4.5. We have argued in eqns. (123) -(126) that one can choose a four-cycle basis $\Gamma_{0}, \Gamma_{\mathcal{B}}, \Gamma_{i}$ of $H_{4}(Y)$ such that the triple intersection form reads

$$
I_{3}=243 \Gamma_{0}^{3}+9 \Gamma_{\mathcal{B}}^{3}+\sum_{i} \Gamma_{i}^{3}
$$

The $\Gamma_{\mathcal{B}}$ and $\Gamma_{i}$ are del Pezzo surfaces, and hence are the candidate $D_{d P}$ for an appropriate orientifold projection. We use this basis in the expansion of the Kähler form $J=-r_{d P}\left[D_{d P}\right]+\ldots$, where $r_{d P}>0$ in the Kähler cone. The coordinate $T_{d P}$ associated to $D_{d P}$ is then given by

$$
\operatorname{Re} T_{d P}=\frac{1}{2} e^{-\phi} \int_{D_{d P}} J \wedge J \sim r_{d P}^{2}
$$

and the Kähler potential for the fields $G^{i}, T_{I}$ takes the form

$$
K=-2 \ln \left(\mathcal{V}_{\text {red }}-\left(T_{d P}+\bar{T}_{d P}\right)^{\frac{3}{2}}\right),
$$

where we have used eq. (115). The important point is that $\mathcal{V}_{\text {red }}$ is independent of the moduli $T_{d P}$ and only depends on the remaining $G^{i}, T_{I}$. We can also evaluate the Killing vector in the basis $\Gamma_{0}, \Gamma_{\mathcal{B}}, \Gamma_{i}$ and find that it diagonalises in the $T_{d P}$ direction with the only non-trivial contribution

$$
X_{d P}=\int_{D_{d P}}\left[D_{d P}\right] \wedge c_{1}\left(\widetilde{L}_{d P}\right)
$$

Using these equations it is straightforward to evaluate the D-term

$$
\xi_{d P} \sim r_{d P}
$$

which thus has to vanish for a supersymmetric vacuum $\xi_{d P}=0$. This implies that we are taken to the point $r_{d P} \rightarrow 0$, where the size of the del Pezzo surface becomes of order string scale. 
Entering a small volume regime implies that our classical analysis is no longer valid and additional corrections need to be included. In particular, as in the underlying $\mathcal{N}=2$ theory, world-sheet instantons will correct the expressions. However, these corrections will not alter the fact that $\partial_{T_{d P}} K \sim r_{d P}$ but rather correct the definition of the $\mathcal{N}=1$ coordinate $T_{d P}$ [63]. Thus, if one still uses the eq. (131) for the Killing vector one is unavoidably driven to the point where $r_{d P}$ is small. This is precisely the regime, where for the local building the techniques of quiver gauge theories on $\mathrm{dP}_{r}$ singularities are relevant [75, 76, 29, 30, 77]. Thus, the global models we presented provide a concrete embedding of these local constructions.

The question now is how general our findings are. One might naively think that whenever one has shrinkable $\mathrm{dP}_{r}, r \leq 8$ surfaces the triple intersection form has the swiss-cheese form eq. (127) so that for GUT branes on these cycles, one is driven to the quiver locus. In Section 7 we will present another class of del Pezzo transitions, based on the Quintic, in which we will instead find mutually intersecting $\mathrm{dP}_{r}$ surfaces, which therefore do not diagonalise the triple intersection form. Therefore, it is not the shrink-ability of the del Pezzo surfaces but rather the swiss-cheese form of the triple intersection form which is responsible for the D-term minimisation at the quiver locus.

\section{$5 \quad$ A GUT Model on $\mathrm{M}_{2}^{\left(\mathrm{dP}_{9}\right)^{2}}$}

In this section we investigate whether the simple geometries with orientifold involutions introduced in the last section are already sufficient to construct realistic, globally consistent intersecting D7-brane GUT models. We work out one toy example in some detail which exemplifies the necessary steps to build a realistic model. This will illustrate the important role played by the structure of the manifold to satisfy the constraints from Table 3. The discussion of this section also serves as a preparation for the construction of two three-generation GUT models in Section 6 on related geometries.

Concretely, we consider the Calabi-Yau manifold $M_{2}^{\left(\mathrm{dP}_{9}\right)^{2}}$ of Subsection 4.3 and choose as the orientifold involution $\Omega \sigma(-1)^{F_{L}}$ with $\sigma: x_{3} \rightarrow-x_{3}$. As discussed previously, the fixed point locus consists of the disjoint divisors $\pi^{*}\left(E_{1}\right)$ and $\pi^{*}(l-$ $\left.E_{2}\right)$ and four additional fixed points. To cancel the D7-brane tadpole eq. (13), we introduce D7-branes on the divisors

$$
D_{a}=\pi^{*}\left(E_{1}\right), \quad D_{b}=\pi^{*}\left(l-E_{1}-E_{2}\right), \quad D_{c}=\pi^{*}\left(l-E_{2}\right),
$$


and denote the corresponding embeddings $\iota_{j}: D_{j} \hookrightarrow Y$. Recall from Subsection 4.3 that $D_{a}, D_{b}$ are $\mathrm{dP}_{9}$ surfaces while $D_{c}$ is a $\mathrm{K} 3$ surface. The O7-tadpole $8\left(\pi^{*}(l-\right.$ $\left.\left.E_{2}\right)+\pi^{*}\left(E_{1}\right)\right)$ is cancelled by three stacks of D7-branes with multiplicities

$$
N \times D_{a}+(N-4) \times D_{b}+(8-N) \times D_{c}, \quad N=4,5,6,7,8
$$

together with their orientifold images wrapping the same divisors. The resulting gauge group is $S O(2 N) \times S P(2 N-8) \times S O(16-2 N)$. Note that the last stack has vanishing intersection with the first two stacks, and will be hidden from the visible sector. The next step is to break the first two gauge groups by turning on non-trivial line-bundles in $U(N) \times U(N-4)$.

\subsection{The Chiral Model}

For now, let us first focus on the chiral sector of the theory and solely compute chiral indices. The computation of the entire cohomology classes is postponed to Subsection 5.4. As our initial Ansatz for the line bundles on the divisors, we will pick all three to be restrictions of global line bundles,

$$
\begin{aligned}
& L_{a}=\iota_{a}^{*} \mathcal{O}_{Y}\left(k \mathcal{B}+\pi^{*}\left(\widetilde{\eta}_{a}\right)\right), \\
& L_{b}=\iota_{b}^{*} \mathcal{O}_{Y}\left(k \mathcal{B}+\pi^{*}\left(\widetilde{\eta}_{b}\right)\right), \\
& L_{c}=\iota_{c}^{*} \mathcal{O}_{Y}\left(m \mathcal{B}+\pi^{*}\left(\eta_{c}\right)\right) .
\end{aligned}
$$

In the following, we will be forced to modify this Ansatz by line bundles that are trivial in $H^{2}(Y, \mathbb{Z})$. However, this changes only the vector-like pairs but not the chiral spectrum.

Since $D_{a}$ and $D_{b}$ are not Spin, it is convenient to explicitly split off a factor $\sqrt{K}$ from $\widetilde{\eta}_{a, b}$. Note that one can rewrite

$$
\sqrt{K_{D_{a}}}=\sqrt{\mathcal{O}_{D_{a}}(-f)}=\iota_{a}^{*} \sqrt{\mathcal{O}_{Y}\left(-D_{a}\right)}=\iota_{a}^{*} \mathcal{O}_{Y}\left(-\frac{1}{2} \pi^{*}\left(E_{1}\right)\right)
$$

and similarly for $D_{b}$. Hence, let us set

$$
\widetilde{\eta}_{a}=\eta_{a}-\frac{1}{2} E_{1}, \quad \widetilde{\eta}_{b}=\eta_{b}-\frac{1}{2}\left(l-E_{1}-E_{2}\right) .
$$

With these definitions, we have parametrised the line bundles by

$$
k, l, m \in \frac{1}{2} \mathbb{Z}, \quad \eta_{a} \in H_{2}\left(D_{a}, \frac{1}{2} \mathbb{Z}\right), \quad \eta_{b} \in H_{2}\left(D_{b}, \frac{1}{2} \mathbb{Z}\right), \quad \eta_{c} \in H_{2}\left(D_{c}, \frac{1}{2} \mathbb{Z}\right) .
$$


Moreover, for vanishing $B_{+}$-field all have to be integral. For non-zero discrete $B_{+^{-}}$ flux, they have to satisfy the quantisation condition eq. (8).

In view of the rules form Table 1 it is straightforward to compute the resulting chiral spectrum, and we list it in Table 7. Let us make a couple of remarks concerning

\begin{tabular}{|c||c|c|c|}
\hline \hline number & $U(N)$ & $U(N-4)$ & $U(8-N)$ \\
\hline \hline$-2 k$ & $\square_{(2)}$ & 1 & 1 \\
$-2 k$ & 1 & $\square(2)$ & 1 \\
$-2 k$ & $\bar{\square}_{(-1)}$ & $\bar{\square}_{(-1)}$ & 1 \\
\hline \hline
\end{tabular}

Table 7: Chiral spectrum for intersecting D7-brane model. The indices denote the $U(1)$ charges.

this spectrum: The third stack does not carry any chiral modes and is completely hidden from the first two. Moreover, the cubic $S U(N)$ and $S U(N-4)$ anomalies are indeed cancelled. Analysing the Abelian and mixed Abelian-non-Abelian anomalies, we find that the linear combination $U(1)_{X}=\frac{1}{N} U(1)_{a}+\frac{1}{N-4} U(1)_{b}$ is anomaly-free. However, due to the Green-Schwarz mass terms, it can nevertheless receive a mass by mixing with an axionic mode. In fact, this is the case as long as the first Chern classes of the line bundles are independent as elements in $H^{2}(Y)$ [54], and there is no massless $U(1)$ prior to breaking $S U(5)$ to the Standard Model. Intriguingly, for $N=5$ we get an $S U(5)$ model with $N_{\text {gen }}=-2 k$ generations of Standard Model particles. The $2 k$ states in the symmetric representation of $U(1)_{b}$ carry the quantum numbers of right-handed neutrinos. So far we have not required D-term supersymmetry of the configuration. For this, one has to ensure that one can choose the Kähler moduli inside the Kähler cone. We will come to this in Subsection 5.3.

\subsection{D3-Brane Tadpole and K-Theory Constraints}

In this subsection, we now investigate the D3-brane tadpole cancellation condition in some more detail. As we have seen, this condition plays no role for the cancellation of the non-Abelian anomalies. In absence of three-form flux the general equation (22) is evaluated to be

$$
N_{D 3}+N_{\text {gauge }}=10
$$


where the contribution from the $U(1)$ fluxes on the D7-branes

$$
\begin{aligned}
N_{\text {gauge }}= & -\frac{1}{2} \sum_{i} N_{i} \int_{D_{i}} c_{1}^{2}\left(L_{i}\right) \\
= & k\left[(N-2) k-N E_{1} \cdot \widetilde{\eta}_{a}-(N-4)\left(l-E_{1}-E_{2}\right) \cdot \widetilde{\eta}_{b}\right] \\
& +m\left[(8-N) m-(8-N)\left(l-E_{2}\right) \cdot \eta_{c}\right]
\end{aligned}
$$

Clearly, for half-integer $k, m$ and pull-back classes $\pi^{*}\left(\eta_{a, b, c}\right)$, this is not always an integer. In this case we simply cannot cancel this tadpole by introducing an integer number of filler D3-branes. Only models with $N_{\text {gauge }} \in \mathbb{Z}$ can be tad-pole free.

Let us now turn to the K-theory constraints. As discussed, these can be determined by the SP-probe brane argument and cancellation of the Witten anomaly. In general, the identification of all potential SP-branes is not an easy task and it is often hard to decide if one has not missed a $\mathbb{Z}_{2}$ constraint. As pointed out previously, since we do not have a CFT description, it not straightforward to decide whether a invariant four-cycle supports $S O$ or $S P$ Chan-Paton factors. Our strategy is to start from the branes on the O-planes, that is, $\pi^{*}\left(E_{1}\right)$ and $\pi^{*}\left(l-E_{2}\right)$, from which we know that they carry $S O$ Chan-Paton factors. A four-cycle with locally four Neumann-Dirichlet boundary conditions relative to the O-planes is expected to carry $S P$ Chan-Paton factors. Consider the divisor $\pi^{*}\left(l-E_{1}-E_{2}\right)$, which wraps the toroidal fibre and intersects $\pi^{*}\left(E_{1}\right)$ in a point in the four-dimensional base $\mathcal{B}$. There, we expect that a brane wrapped on $\pi^{*}\left(l-E_{1}-E_{2}\right)$ carries $S P$ Chan-Paton factors. This identification is further supported by the chiral spectrum in Table 7, where the $a^{\prime} a$ sector leads to fields in the anti-symmetric representation, whereas the $b^{\prime} b$ sector leads to symmetric ones.

This line of reasoning identifies three 4-cycles supporting symplectic Chan-Paton factors, namely

$$
\mathcal{B}, \quad \pi^{*}\left(E_{2}\right), \quad \pi^{*}\left(l-E_{1}-E_{2}\right) .
$$

All of these divisors are not Spin and, therefore, they have to carry a half-integral bundle to comply with the Freed-Witten quantisation condition. As usual, under the action of $\Omega$ the field strength gets reflected, in which case the candidates in eq. (141) actually carry $U(N)$ Chan-Paton factors. However, by turning on quantised B-flux through some cycles in $H_{2}(Y, \mathbb{Z})$, the quantisation conditions on the divisors can change, in which case vanishing gauge flux is allowed resulting in a non-trivial $\mathbb{Z}_{2}$ K-theory constraint. 


\subsection{D-Flatness}

According to the general discussion in Subsection 2.4, the FI-terms for $U(1)_{a}$ and $U(1)_{b}$ are given by

$$
\begin{aligned}
\xi_{a} & \sim k\left(r_{1}-r_{\mathcal{B}}\right)+r_{\mathcal{B}} E_{1} \cdot \widetilde{\eta}_{a} \\
\xi_{b} & \sim k\left(r_{l}-r_{1}-r_{2}-r_{\mathcal{B}}\right)+r_{\mathcal{B}}\left(l-E_{1}-E_{2}\right) \cdot \widetilde{\eta}_{b}, \\
\xi_{c} & \sim m\left(r_{l}-r_{2}-2 r_{\mathcal{B}}\right)+r_{\mathcal{B}}\left(l-E_{2}\right) \cdot \eta_{c}
\end{aligned}
$$

where we have expanded

$$
J=r_{\mathcal{B}} \mathcal{B}+r_{l} \pi^{*}(l)-r_{1} \pi^{*}\left(E_{1}\right)-r_{2} \pi^{*}\left(E_{2}\right) .
$$

Since we do not want to give VEVs to Standard Model fields charged under $U(1)_{a}$ respectively $U(1)_{b}$, we have to require that the two FI-terms eq. (142) vanish. We have to make sure that the resulting constraints define a plane inside the Kähler cone of this triangulation

$$
r_{\mathcal{B}}>0, \quad r_{1}-r_{\mathcal{B}}>0, \quad r_{2}-r_{\mathcal{B}}>0, \quad r_{l}-r_{1}-r_{2}-r_{\mathcal{B}}>0
$$

However, we are actually interested in a slightly weaker condition. Since the top Yukawa coupling $10105_{H}$ is of order one, the coupling-generating instanton necessarily has to be in the non-perturbative regime. In fact, we would like to achieve vanishing (classical) volume of the 4-cycle wrapped by the instanton, corresponding to a particular boundary of the Kähler cone. Note that world-sheet instanton corrections are expected to eventually fix the size at the order of the string scale. Of course, for the model to make sense the gauge couplings of all space-time filling D7-branes should stay finite, which can be achieved in presence of non-trivial gauge flux.

Note that, even if one has satisfied the D7- and D5-brane tadpole cancellation conditions guaranteeing already cancellation of non-Abelian anomalies, the extra conditions of

- integer D3-brane tadpole contribution of the gauge fluxes on the D7-branes,

- a number of $\mathbb{Z}_{2}$ K-theory constraints, and

- satisfying the D-flatness conditions inside or at most on the boundary of the Kähler cone 
provide further strong constraints. On the Calabi-Yau threefold $M_{2}^{\left(\mathrm{dP}_{9}\right)^{2}}$, we have not succeeded in realising a $S U(5)$ GUT model with an odd number of generations. One either does not satisfy the first condition above or lands on an unacceptable boundary of the Kähler cone in the sense that the volume of the Calabi-Yau manifold vanishes. The best example we have found by our manual search on $M_{2}^{\left(\mathrm{dP}_{9}\right)^{2}}$ will be detailed in the next section. Having said this, we will resolve the described problems for concrete examples on the related manifold $M_{2}^{\left(\mathrm{dP}_{8}\right)^{2}}$ in Section 6.

\subsection{Globally Consistent Model}

We now fix $N=5$, leading to a gauge group $U(5) \times U(1) \times S O(6)$. For an odd number of generations we would have to consider an orientifold without vector structure but with discrete NS-NS two-form flux $\int_{F} B=\frac{1}{2}$. As mentioned, in this case we did not succeed in satisfying integer D3-brane tadpole contribution of the gauge flux and D-flatness inside the Kähler cone. Therefore, we will settle for a model with an even number of generations and choose

$$
c_{1}(B)=\frac{1}{2} \pi^{*}\left(E_{1}\right) .
$$

In particular, this satisfies $\int_{F} B=0$ and, therefore, $k \in \mathbb{Z}$ must be integral.

Now we choose the line bundles on the three stacks of D7-branes to be

$$
\begin{aligned}
L_{a} & =\iota_{a}^{*} \mathcal{O}_{Y}\left(-\mathcal{B}-\pi^{*}\left(E_{1}\right)\right), \\
L_{b} & =\iota_{b}^{*} \mathcal{O}_{Y}(-\mathcal{B}), \\
L_{c} & =\iota_{c}^{*} \mathcal{O}_{Y} .
\end{aligned}
$$

For explicitness, let us check the quantisation eq. (86). On the $\mathrm{dP}_{9}$ surfaces, we will use the standard basis

$$
H_{2}\left(\mathrm{dP}_{9}, \mathbb{Z}\right)=\operatorname{span}_{\mathbb{Z}}\left\{l, e_{1}, \ldots, e_{9}\right\} .
$$

Each such surface $D_{a}, D_{b}$ is elliptically fibred with fibre class $f=3 l-\sum e_{i}$ and zero-section

$$
\mathcal{B} \cap D_{a}=e_{9} \in H_{2}\left(D_{a}, \mathbb{Z}\right), \quad \mathcal{B} \cap D_{b}=e_{9} \in H_{2}\left(D_{b}, \mathbb{Z}\right) .
$$

${ }^{23}$ That is, $N_{a}=5, N_{b}=1$, and $N_{c}=3$. 
Noting that the $B$-flux eq. (145) restricts trivially on the four-cycle $D_{c}$, one finds that

$$
\begin{aligned}
& \begin{array}{rcc}
D_{j}: & c_{1}\left(L_{j}\right)-\left.B\right|_{D_{j}}+\frac{1}{2} c_{1}\left(K_{D_{j}}\right) & \in H_{2}\left(D_{j}, \mathbb{Z}\right) \\
\hline D_{a}=\pi^{*}\left(E_{1}\right): & -e_{9}+f-\left(-\frac{1}{2} f\right)+\frac{1}{2}(-f) & \in H_{2}\left(D_{a}, \mathbb{Z}\right)
\end{array} \\
& D_{b}=\pi^{*}\left(l-E_{1}-E_{2}\right): \quad-e_{9} \quad-\quad \frac{1}{2} f \quad+\quad \frac{1}{2}(-f) \quad \in H_{2}\left(D_{b}, \mathbb{Z}\right) \\
& D_{c}=\pi^{*}\left(l-E_{2}\right): \quad 0 \quad-\quad 0 \quad+\quad 0 \quad \in H_{2}\left(D_{c}, \mathbb{Z}\right),
\end{aligned}
$$

and the bundles are, indeed, correctly quantised. For this choice, the contribution to the D3-brane tadpole is

$$
N_{\text {gauge }}=-\frac{1}{2} \sum_{i} N_{i} \int_{D_{i}} c_{1}^{2}\left(L_{i}\right)=8
$$

and we can cancel this tadpole, for example, by two dynamical D3-brane carrying $S P(4)$ gauge group. However, according to eq. (59), this cannot be the final answer as we will get an additional contribution from the $U(1)_{Y}$ flux.

A D7-brane wrapping the divisor $\pi^{*}\left(l-E_{1}-E_{2}\right)$ now carries integer quantised gauge flux. Therefore, the gauge bundle can be chosen to be trivial leading to an $S P$-brane. For such a probe brane we get a Witten anomaly respectively expect a K-theory constraint. It is easy to see that the above choice of branes and line bundles gives indeed a even number of fundamental $S P$-representations. The $B$-flux eq. (145) does not restrict to the other candidate $S P$ cycles, $\mathcal{B}$ and $\pi^{*}\left(E_{2}\right)$ so that a trivial line bundle does not exist. Consequently no further conditions arise.

The supersymmetry conditions become

$$
\xi_{a}=r_{1}-2 r_{\sigma}=0, \quad \xi_{b}=r_{l}-r_{1}-r_{2}-r_{\sigma}=0 .
$$

The first condition can be satisfied inside the Kähler cone whereas the second condition lies on the boundary of the Kähler cone where the 2-cycle $C=l-E_{1}-E_{2}$ inside $\mathrm{dP}_{2}$ has zero size. As we will explain in detail in the following, this is exactly as desired, and there exists a D3-instanton wrapping $\pi^{*}\left(l-E_{1}-E_{2}\right)$ and generating the top-Yukawa couplings. Due to the non-trivial line bundle on the brane $D_{b}$, we find for the $U(1)$ gauge coupling on this brane

$$
\frac{1}{g_{b}^{2}} \sim-\int_{D_{b}} c_{1}^{2}\left(L_{b}\right)=O(1)
$$

which stays finite but, thankfully, leaves the perturbative regime. 


\section{Non-chiral SU(5) spectrum}

We now move forward and compute the vector-like matter spectrum as well. First, we turn to the GUT brane. To compute the relevant bundle cohomology groups, we first evaluate the pull-back in the definition of the line bundles eq. (146). One obtains

$$
\begin{aligned}
L_{a} & =\iota_{a}^{*} \mathcal{O}_{Y}\left(-\mathcal{B}-\pi^{*}\left(E_{1}\right)\right)=\mathcal{O}_{D_{a}}\left(\left(-\mathcal{B}-\pi^{*}\left(E_{1}\right)\right) \cap D_{a}\right) \\
& =\mathcal{O}_{D_{a}}\left(-e_{9}+f\right), \\
L_{b} & =\mathcal{O}_{D_{b}}\left(-e_{9}\right), \\
L_{c} & =\mathcal{O}_{D_{c}} .
\end{aligned}
$$

For the $S U(5)$ matter, we now have to compute the cohomology of powers of $L_{a}$. Using Appendix C, we easily find

$$
H^{*}\left(\mathrm{dP}_{9}, L_{a}^{\vee} \otimes L_{a}^{\vee}\right)=(0,4,0), \quad H^{*}\left(\mathrm{dP}_{9}, L_{a} \otimes L_{a}\right)=(0,6,0) .
$$

Thus we get two chiral and four vector-like pairs of matter fields in the anti-symmetric representation 10 of $S U(5)$.

Next, let us consider the states in the symmetric representation 1 of $U(1)$, that is, the right-handed neutrinos. Since the bundle $\left(L_{b}^{\vee}\right)^{2}$ has sections, we get unwelcome non-trivial elements in $\operatorname{Ext}^{0}\left(\iota_{*} L_{b}^{\vee}, \iota_{*} L_{b}\right)$ which would render the theory inconsistent. In order to get rid of these, we now use the freedom to twist this pull-back bundle by a bundle $R_{b}$ whose push-forward is trivial in $Y$. In particular, we pick

$$
R_{a}=\mathcal{O}_{D_{a}}, \quad R_{b}=\mathcal{O}_{D_{b}}\left(-e_{1}+e_{2}\right), \quad R_{c}=\mathcal{O}_{D_{c}}
$$

and replace the bundle on $D_{i}, i=\{a, b, c\}$, with the tensor product

$$
L_{i} \longrightarrow \widetilde{L}_{i}=L_{i} \otimes R_{i}
$$

As explained previously, this modification does not change the chiral matter content. However, the twisting with $R_{b}$ yields an extra contribution to the D3-tadpole eq. (150), which is now

$$
N_{\text {gauge }}=-\sum_{i} N_{i} \int_{D_{i}} \operatorname{ch}_{2}\left(L_{i} \otimes R_{i}\right)=9 .
$$

The spectrum is, now,

$$
H^{*}\left(\mathrm{dP}_{9},\left(L_{b}^{\vee}\right)^{2} \otimes\left(R_{b}^{\vee}\right)^{2}\right)=(0,4,0), \quad H^{*}\left(\mathrm{dP}_{9},\left(L_{a}\right)^{2} \otimes\left(R_{b}\right)^{2}\right)=(0,6,0),
$$


yielding two chiral and two vector-like pairs.

Finally, consider the matter fields in the $\overline{\mathbf{5}}$ representation, which are localised on the curve

$$
D_{a} \cap D_{b}=F,
$$

that is, on an elliptic fibre $\mathbb{P}_{1,2,3}[6] \subset Y$. We first need to pull-back the bundles via the inclusion $\iota_{F}: F \rightarrow D_{a}$ and $D_{b}$, respectively. Note that the specific fibre $F$ is, viewed as a curve in $D_{a}$ or $D_{b}$, in the fibre class $f$. In order to label its intersection points with the sections $e_{1}, \ldots, e_{9}$, let us define

$$
\begin{gathered}
p_{1}=\iota_{a}\left(e_{1}\right) \cap F, \quad p_{2}=\iota_{a}\left(e_{2}\right) \cap F, \quad \ldots, \quad p_{8}=\iota_{a}\left(e_{8}\right) \cap F, \\
p_{1}^{\prime}=\iota_{b}\left(e_{1}\right) \cap F, \quad p_{2}^{\prime}=\iota_{b}\left(e_{2}\right) \cap F, \quad \ldots, \quad p_{8}^{\prime}=\iota_{b}\left(e_{8}\right) \cap F, \\
0=\iota_{a}\left(e_{9}\right) \cap F=\iota_{b}\left(e_{9}\right) \cap F=\mathcal{B} \cap F .
\end{gathered}
$$

Here we have implicitly fixed some of the complex structure moduli of the manifold such that indeed the sections of $D_{a}$ and $D_{b}$ intersect $F$ in the same points. Using this notation, we obtain

$$
\begin{aligned}
\left.L_{a}\right|_{F} & =\iota_{F}^{*} L_{a}=\mathcal{O}_{F}\left(\left(-e_{9}+f\right) \cap F\right)=\mathcal{O}_{F}(-0), \\
\left.\left(L_{b} \otimes R_{b}\right)\right|_{F} & =\mathcal{O}_{F}\left(-0-p_{1}^{\prime}+p_{2}^{\prime}\right) .
\end{aligned}
$$

With $K_{F}=\mathcal{O}_{F}$ we can easily compute the $\overline{\mathbf{5}}$-spectrum, and obtain

$$
\begin{aligned}
H^{*}\left(F, \iota_{F}^{*}\left(L_{a}^{\vee} \otimes\left(L_{b} \otimes R_{b}\right)^{\vee}\right)\right) & =H^{*}\left(F, \mathcal{O}_{F}\left(0+0+p_{1}^{\prime}-p_{2}^{\prime}\right)\right) \\
& =H^{*}\left(F, \mathcal{O}_{F}(2 \text { pts. })\right)=(2,0) .
\end{aligned}
$$

Hence, we get precisely 2 chiral fields in the anti-fundamental representation. The Higgs $\mathbf{5}_{H}+\overline{\mathbf{5}}_{H}$-spectrum, on the other hand, is determined by

$$
H^{*}\left(F, \iota_{F}^{*}\left(L_{a}^{\vee} \otimes\left(L_{b} \otimes R_{b}\right)\right)\right)=H^{*}\left(F, \mathcal{O}_{F}\left(-p_{1}^{\prime}+p_{2}^{\prime}\right)\right)=(0,0) .
$$

One might worry that there is no candidate $\mathbf{5}_{H}-\overline{\mathbf{5}}_{H}$ pair giving rise to the Higgs after symmetry breaking; However, as we will see in the following, turning on a suitable $L_{Y}$ flux will generate one vector-like pair as desired.

\section{Instanton effects}

A Euclidean D3-brane wrapping the divisor $\pi^{*}\left(l-E_{1}-E_{2}\right)$ with trivial line bundle is of $O(1)$ type and clearly rigid. Therefore, this instanton is a candidate to generate 
the $10105_{\mathbf{H}}$ Yukawa couplings. For the chiral charged matter zero modes, we indeed get $I_{a \text {,inst }}=1$ and $I_{b \text {,inst }}=-1$. Therefore, the necessary condition eq. (45) for the generation of the top Yukawa coupling is satisfied. Moreover, the line bundle on the divisor $D_{b}=\pi^{*}\left(l-E_{1}-E_{2}\right)$ has first Chern class $c_{1}\left(L_{b} \otimes R_{b}\right)=-e_{9}-e_{1}+e_{2}$, and therefore

$$
H^{*}\left(D_{b}, L_{b} \otimes R_{b}\right)=(0,1,0), \quad H^{*}\left(D_{b}, L_{b}^{\vee} \otimes R_{b}^{\vee}\right)=(0,0,0) .
$$

This implies $\operatorname{Ext}^{*}\left(L_{b} \otimes R_{b}, \mathcal{O}_{D_{b}}\right)=(0,1,0,0)$ and shows that there exists precisely one chiral zero mode $\lambda_{b}$ without any additional vector-like pairs. Since the instanton intersects the brane $D_{a}$ over the fibre curve, we find exactly one chiral zero mode $\lambda_{a}$. Moreover, the D-term constraint for the brane $D_{b}$ has fixed the Kähler moduli such that the instanton action goes to zero; it follows that leaving (locally) the perturbative regime, the top Yukawa couplings are really of order one.

To generate Majorana neutrino masses, one needs an $O(1)$ instanton intersecting only the brane stack $D_{b}$. A candidate would be a Euclidean D3-brane wrapping the divisor $\pi^{*}\left(E_{2}\right)$. However, since this divisor is not Spin, it is not of $O(1)$ but $U(1)$ type.

\section{Non-chiral $\mathrm{SU}(3) \times \mathrm{SU}(2) \times \mathrm{U}(1)_{\mathrm{Y}}$ spectrum}

Finally, let us break the $S U(5)$ gauge symmetry to the Standard Model. To do so, we will turn on $U(1)_{Y}$ gauge flux supported on a curve in $H_{2}\left(D_{a}, \mathbb{Z}\right)$ which is a boundary on $Y$. In particular, we pick

$$
\mathcal{L}_{a}=L_{a}, \quad \mathcal{L}_{Y}=\mathcal{O}_{D_{a}}\left(-e_{1}+e_{2}\right) .
$$

The $\mathcal{L}_{Y}$ bundle has vanishing cohomology classes on $D_{a}=\mathrm{dP}_{9}$, and, therefore, no exotics are introduced. The contribution to the D3-tadpole from this flux is

$$
N_{\text {gauge }}^{Y}=-\int_{D_{a}} c_{1}^{2}\left(\mathcal{L}_{Y}\right)=2
$$

The combined gauge flux contribution to the D3 tadpole is $N_{\text {gauge }}=9+2=11$, overshooting by one unit. Therefore, to cancel this tadpole, one needs to introduce one dynamical anti-D3-brane.

The relevant cohomology classes for the descendants of the antisymmetric representation 10 of $S U(5)$ are listed in Table 8. Note that, of course, the chiral matter 


\begin{tabular}{|c|c|c|c|c|}
\hline GUT & SM Field & Cohomology & chiral & vector \\
\hline \multirow{3}{*}{10} & $(\overline{\mathbf{3}}, \mathbf{1})_{-4_{Y}}$ & $\begin{aligned} H^{*}\left(D_{a},\left(\mathcal{L}_{a}^{\vee}\right)^{2}\right) & =(0,4,0) \\
H^{*}\left(D_{a}, \mathcal{L}_{a}^{2}\right) & =(0,6,0)\end{aligned}$ & 2 & $4+4$ \\
\hline & $(3,2)_{1_{Y}}$ & $\begin{array}{r}H^{*}\left(D_{a},\left(\mathcal{L}_{a}^{\vee}\right)^{2} \otimes \mathcal{L}_{Y}^{-1}\right)=(0,5,0) \\
H^{*}\left(D_{a}, \mathcal{L}_{a}^{2} \otimes \mathcal{L}_{Y}\right)=(0,7,0)\end{array}$ & 2 & $5+5$ \\
\hline & $(\mathbf{1}, \mathbf{1})_{6_{Y}}$ & $\begin{array}{r}H^{*}\left(D_{a},\left(\mathcal{L}_{a}^{\vee}\right)^{2} \otimes \mathcal{L}_{Y}^{-2}\right)=(0,8,0) \\
H^{*}\left(D_{a}, \mathcal{L}_{a}^{2} \otimes \mathcal{L}_{Y}^{2}\right)=(0,10,0)\end{array}$ & 2 & $8+8$ \\
\hline 1 & $(\mathbf{1}, \mathbf{1})_{O_{Y}}$ & $\begin{aligned} H^{*}\left(D_{b},\left(L_{b}^{\vee} \otimes R_{b}^{\vee}\right)^{2}\right) & =(0,4,0) \\
H^{*}\left(D_{b}, L_{b}^{2} \otimes R_{b}^{2}\right) & =(0,6,0)\end{aligned}$ & 2 & $4+4$ \\
\hline$\overline{5}$ & $\begin{array}{l}(\overline{\mathbf{3}}, \mathbf{1})_{2_{Y}} \\
(\mathbf{1}, \mathbf{2})_{-3_{Y}}\end{array}$ & $\begin{array}{l}\text { eq. } 168 \mathrm{a}) \\
\text { eq. } 1168 \mathrm{~b}\end{array}$ & $\begin{array}{l}2 \\
2\end{array}$ & $\begin{array}{l}0 \\
0\end{array}$ \\
\hline $\mathbf{5}_{H}+\overline{\mathbf{5}}_{H}$ & $\begin{array}{l}(\mathbf{3}, \mathbf{1})_{-2_{Y}} \\
(\mathbf{1}, \mathbf{2})_{3_{Y}}\end{array}$ & $\begin{aligned} H^{*}\left(F, \mathcal{O}_{F}\left(-p_{1}^{\prime}+p_{2}^{\prime}\right)\right) & =(0,0) \\
H^{*}\left(F, \mathcal{O}_{F}\right) & =(1,1)\end{aligned}$ & $\begin{array}{l}0 \\
0\end{array}$ & $\begin{array}{c}0 \\
1+1\end{array}$ \\
\hline
\end{tabular}

Table 8: Spectrum for the orientifold model in Section 5. The indices denote the $U(1)$ charges. 
does not change; only extra vector-like pairs of matter fields appear. We now turn toward the matter fields in the anti-fundamental representation $\overline{\mathbf{5}}$ as well as the Higgs, both of which are localised on the intersection curve $F$. In addition to eq. (161), we have

$$
\iota_{F}^{*} \mathcal{L}_{Y}=\left.\mathcal{L}_{Y}\right|_{F}=\mathcal{O}_{F}\left(-p_{1}+p_{2}\right) .
$$

First, note that the $\overline{\mathbf{5}}$-spectrum is unchanged since

$$
\begin{gathered}
H^{*}\left(F, \iota_{F}^{*}\left(\mathcal{L}_{a}^{\vee} \otimes L_{b}^{\vee} \otimes R_{b}^{\vee}\right)\right)=H^{*}\left(\mathcal{O}_{F}\left(0+0+p_{1}^{\prime}-p_{2}^{\prime}\right)\right)=(2,0), \\
H^{*}\left(F, \iota_{F}^{*}\left(\mathcal{L}_{a}^{\vee} \otimes L_{b}^{\vee} \otimes R_{b}^{\vee} \otimes \mathcal{L}_{Y}^{\vee}\right)\right)=H^{*}\left(\mathcal{O}_{F}\left(2 \cdot 0+p_{1}+p_{1}^{\prime}-p_{2}-p_{2}^{\prime}\right)\right)=(2,0) .
\end{gathered}
$$

More interesting is the Higgs spectrum, which is determined by

$$
\iota_{F}^{*}\left(\mathcal{L}_{a}^{\vee} \otimes\left(L_{b} \otimes R_{b}\right) \otimes \mathcal{L}_{Y}^{\vee}\right)=\mathcal{O}_{F}\left(p_{1}-p_{1}^{\prime}+p_{2}-p_{2}^{\prime}\right)=\mathcal{O}_{F}(0-q)
$$

for some point $q \in F$. The precise point can be computed using the group law on the elliptic curve $F$, and will depend on the complex structure of $D_{a}, D_{b}$ (and, therefore, $Y)$. We assume that $q=0$, which happens on a locus of codimension one in the complex structure moduli space; See also the discussion around eq. (160). In this case,

$$
H^{*}\left(F, \iota_{F}^{*}\left(\mathcal{L}_{a}^{\vee} \otimes\left(L_{b} \otimes R_{b}\right) \otimes \mathcal{L}_{Y}^{\vee}\right)\right)=H^{*}\left(F, \mathcal{O}_{F}\right)=(1,1) .
$$

As desired, we then obtain one pair of Higgs-conjugate Higgs fields. Moreover, the Higgs doublet is, in fact, split from the dangerous colour triplet. The latter is still absent, thanks to

$$
H^{*}\left(F, \iota_{F}^{*}\left(\mathcal{L}_{a}^{\vee} \otimes\left(L_{b} \otimes R_{b}\right)\right)\right)=H^{*}\left(F, \mathcal{O}_{F}\left(-p_{1}^{\prime}+p_{2}^{\prime}\right)\right)=(0,0) .
$$

To summarise, we have defined a simple involution on $M_{2}^{\left(\mathrm{dP}_{9}\right)^{2}}$ which allows for the introduction of a three-stack intersecting D7-brane configuration cancelling the D7-, D5- and D3-brane tadpoles, the latter at the cost of introducing one anti D3-brane, as well as the K-theory tadpoles. We have found an $S U(5)$ GUT-like model with two chiral generations of Standard Model particles and one Higgs-conjugate Higgs pair. Moreover, we have been able to realise the $U(1)_{Y}$ flux gauge symmetry breaking and computed the resulting chiral and non-chiral matter spectrum. The inevitable appearance of the latter is one of the shortcomings of this example. It can be traced back to the fact that our involution acts trivially on the cohomology $H^{2}(Y, \mathbb{Z})$. As a 
consequence, antisymmetric matter is localised not on a curve but on a whole divisor, widening the sources of contributions to the cohomology. By contrast, matter and Higgs are localised on the elliptic fibre of $Y$. Since the Higgs $H_{u}$ and $H_{d}$ are localised on the same curve, we cannot suppress dimension-five proton decay operators. The D-term supersymmetry conditions can be satisfied on the boundary of Kähler moduli space such that a D3-brane instanton realises 10105 Yukawa couplings of order 1. In Table 9 we summarise phenomenologically desirable features of this simple model.

\begin{tabular}{|c||c|c|}
\hline \hline property & mechanism & status \\
\hline \hline globally consistent & tadpoles + K-theory & $\checkmark^{*, * *}$ \\
D-term susy & vanishing FI-terms inside Kähler cone & $\checkmark^{* * *}$ \\
gauge group $S U(5)$ & choice of line bundles & $\checkmark$ \\
3 chiral generations & localisation on curves & - \\
no vector-like matter & choice of line bundles & - \\
1 vector-like of Higgs & rigid 4-cycles, del Pezzo & $\checkmark$ \\
no adjoints & $U(1)_{Y}$ flux on trivial 2-cycles & $\checkmark$ \\
GUT breaking & Wilson lines on $g=1$ curve & $\checkmark$ \\
$3-2$ splitting & local. of $H_{u}, H_{d}$ on disjoint comp. & - \\
3-2 split + no dim=5 p-decay & perturbative & $\checkmark$ \\
$\mathbf{1 0} \overline{\mathbf{5}} \overline{\mathbf{5}}_{H}$ Yukawa & presence of appropriate D3-instanton & $\checkmark$ \\
$\mathbf{1 0} \mathbf{1 0} \boldsymbol{5}_{H}$ Yukawa & presence of appropriate D3-instanton & $-{ }^{* * * * *}$ \\
\hline Majorana neutrino masses
\end{tabular}

Table 9: Summary of SU(5) properties realised in the model of Section 5.

* overshooting in D3-tadpole $\rightarrow 1 \overline{D 3}$ brane

** K-theory to the best of our ability to detect $S P$ cycles

*** realised on acceptable boundary of Kähler moduli space

**** for special choice of complex structure moduli

***** at least not with $O(1)$ instantons 


\section{GUT Model Search}

The model presented in the previous section does not exhibit all properties desirable for a nice string GUT model. However, as for each single shortcoming it is quite clear how to improve on this. The non-trivial task is to achieve this in a globally consistent framework and without loosing the good features already realised. To this end a more systematic search is necessary and beyond the scope of this paper. In this section we provide a couple of manually found models which incorporate some other desirable properties from Table 3, but come short on already realised ones. The two features we focus on in this section are 3 chiral families and the absence of vector-like matter fields.

\subsection{A 3-Generation GUT Model on $\mathrm{M}_{2}^{\left(\mathrm{dP}_{8}\right)^{2}}$}

We now present an example of a GUT model of the type described previously which indeed gives rise to 3 chiral families of Standard Model matter. To this end we consider the manifold $M_{2}^{\left(\mathrm{dP}_{8}\right)^{2}}$ introduced in Subsection 4.3. Since the intersection form eq. (102) differs considerably from the one of the un-flopped Weierstraß phase, eq. (86), it seems plausible that the no-go result for an odd number of generations can be evaded.

We closely follow the philosophy spelt out in Section 5 so that we can be brief. Concretely, consider again a 3-stack model based on the divisors $D_{a}=D_{7}, D_{b}=D_{5}$ and $D_{c}=D_{5}+D_{7}$, wrapped by D7-branes with multiplicities

$$
N \times D_{7}, \quad(N-4) \times D_{5}, \quad(8-N) \times\left(D_{5}+D_{7}\right)
$$

plus their orientifold images, with $N=5$ corresponding to the GUT model we are interested in. The above configuration satisfies the $D 7$-brane tadpole constraint for any $N$.

Let us first define the chiral $S U(5)$ GUT model by parametrising the part of line bundles $L_{a}, L_{b}$ and $L_{c}$ descending from the Calabi-Yau $Y$ as

$$
\begin{aligned}
L_{a} & =\iota_{a}^{*} \mathcal{O}_{Y}\left(a_{1} D_{5}+a_{3} D_{7}\right), \quad L_{b}=\iota_{b}^{*} \mathcal{O}_{Y}\left(\sum_{i=1}^{4} b_{i} D_{i+4}\right), \\
L_{c} & =\iota_{c}^{*} \mathcal{O}_{Y}\left(\sum_{i=1}^{4} c_{i} D_{i+4}\right) .
\end{aligned}
$$


For the correct definition of the bundles it is essential to take into account that this time all three divisors are not Spin. According to the quantisation condition eq. (7) for vanishing B-field the parameters $b_{1}, a_{3}, c_{1}, c_{3}$ are therefore half-integer while $a_{1}, b_{2}, b_{3}, b_{4}, c_{2}, c_{4}$ are integer.

As can be computed from the intersection form eq. (102), the divisors $D_{a}$ and $D_{b}$ still intersect along a genus 1 curve where the 5 and the $\mathbf{5}_{H}+\overline{\mathbf{5}}_{H}$ are localised. Note also that the divisor $D_{c}$ now intersects $D_{a}$ and $D_{b}$ along a genus 0 and a genus 1 curve, respectively. Still there exists no massless exotic matter in the $D_{a}-D_{c}$ sector, even at the vector-like level, while there might appear truly hidden sector matter fields from the $D_{b}-D_{c}$ intersection. This chiral matter is displayed in Table 10. Clearly all non-Abelian anomalies vanish due to cancellation of D7- and absence of D5-brane tadpoles. Note that for general bundles there exist both symmetric and anti-symmetric states under $U(N-4)$. In what follows we specialise to the case $N=5$ corresponding to a GUT model with the first four lines representing the $\mathbf{1 0}$, $\overline{\mathbf{5}}, \mathbf{5}_{\mathbf{H}}+\overline{\mathbf{5}}_{\mathbf{H}}$ and $N_{R}^{c}$.

\begin{tabular}{|c||c|c|c|}
\hline \hline chirality & $U(N)$ & $U(N-4)$ & $U(8-N)$ \\
\hline \hline$-2 a_{1}+2 a_{3}$ & $\boxminus_{(2)}$ & 1 & 1 \\
$-\left(a_{1}+b_{1}\right)+a_{3}+b_{3}$ & $\square_{(-1)}$ & $\square_{(-1)}$ & 1 \\
$-\left(a_{1}-b_{1}\right)+\left(a_{3}-b_{3}\right)$ & $\square_{(-1)}$ & $\square_{(1)}$ & 1 \\
$2\left(-b_{1}+b_{3}\right)$ & 1 & $\square(2)$ & 1 \\
$2\left(-b_{1}+b_{2}+b_{4}\right)$ & 1 & $\square_{(2)}$ & 1 \\
\hline \hline$\left(b_{1}-c_{1}\right)-\left(b_{2}-c_{2}\right)-\left(b_{4}-c_{4}\right)$ & 1 & $\square_{(-1)}$ & $\square(1)$ \\
$\left(b_{1}+c_{1}\right)-\left(b_{2}+c_{2}\right)-\left(b_{4}+c_{4}\right)$ & 1 & $\square_{(-1)}$ & $\square_{(-1)}$ \\
$2\left(-c_{1}+c_{2}+c_{4}\right)$ & 1 & 1 & $\square_{(2)}$ \\
\hline
\end{tabular}

Table 10: Chiral spectrum for intersecting D\%-brane model. The indices denote the $U(1)$ charges. 


\section{Global consistency conditions}

The D3-brane tadpole condition is

$$
N_{D 3}+N_{\text {gauge }}=10,
$$

with $N_{\text {gauge }}$ given by

$$
\begin{aligned}
-\frac{1}{2} \sum_{a} N_{a} c_{1}^{2}\left(L_{a}\right) & =-\frac{5}{2}\left(a_{1}^{2}+a_{3}^{2}-2 a_{1} a_{3}\right)-\frac{3}{2}\left(-c_{1}^{2}+2 c_{1} c_{2}-3 c_{2}^{2}+2 c_{1} c_{4}-c_{4}^{2}\right) \\
& -\frac{1}{2}\left(-2 b_{1}^{2}+2 b_{1} b_{2}-3 b_{2}^{2}+2 b_{1} b_{3}-b_{3}^{2}+2 b_{1} b_{4}-b_{4}^{2}\right) .
\end{aligned}
$$

Note, however, that there will be additional contributions later on from the part of the line bundles trivial on the ambient space $Y$. As in the previous section, due to the simple structure of the orientifold action, the D5-brane tadpoles cancel automatically between the branes and their image.

On the other hand, there can arise K-theory constraints from the 3 invariant divisors $D_{6}, D_{8}$ and $D_{5}$ which may carry symplectic Chan-Paton factors. The divisor $D_{6}$ is the former basis $\mathrm{dP}_{2}$ of the Weierstraß model $M_{2}^{\left(\mathrm{dP}_{9}\right)^{2}}$ with the two $\mathbb{P}^{1} \mathrm{~s}$ removed. As such it is the surface $\mathbb{P}^{2}$ with $K_{D 6}=\mathcal{O}_{D 6}(3)$ and obviously not Spin. This means that, according to eq. (7), the chiral part of a line bundle on $D_{6}$

$$
L_{D_{6}}=\iota^{*} \mathcal{O}_{Y}\left(x_{1} D_{5}+x_{2} D_{6}\right)
$$

can be trivial only for $B=0 \times D_{5}+\frac{1}{2} D_{6}+\ldots$. Otherwise the cycle does not carry symplectic gauge factors and therefore no K-theory constraint arises from $D_{6}$.

The K-theory constraint from $D_{6}$ therefore reads

$$
b_{1}+3 c_{1}-3\left(b_{2}+3 c_{2}\right) \in 2 \mathbb{Z} \quad \text { if } \quad B=0 \times D_{5}+\frac{1}{2} D_{6} .
$$

A similar analysis for $D_{8}$ and $D_{5}$ yields the two additional constraints

$$
\begin{gathered}
b_{1}+3 c_{1}-\left(b_{4}+3 c_{4}\right) \in 2 \mathbb{Z} \quad \text { if } B=0 \times D_{5}+\frac{1}{2} D_{8}, \\
5 a_{1}-5 a_{3}-2\left(b_{1}+3 c_{1}\right)+\left(b_{2}+3 c_{2}\right)\left(b_{4}+3 c_{4}\right)+3\left(c_{1}-c_{3}\right) \in 2 \mathbb{Z} \\
\text { if } \quad B=\frac{1}{2} D_{5}+0 \times\left(D_{6}+D_{7}+D_{8}\right) .
\end{gathered}
$$

In all other cases the K-theory constraints from these three divisors are trivial. 


\section{D-term supersymmetry constraints}

To determine the D-term supersymmetry conditions we have to expand the Kähler form $J$ in terms of the generators of the full Kähler cone and evaluate the FayetIliopoulos terms. For our purposes it will be sufficient to restrict our attention to the Kähler subcone corresponding to the vectors $K_{i}$ displayed in eq. (101) and take

$$
J=\sum_{i} r_{i} K_{i}, \quad r_{i} \in \mathbb{R}_{+}
$$

As discussed around eq. (101), it suffices to check if the associated FI-terms

$$
\begin{aligned}
& \xi_{a} \simeq\left(a_{1}-a_{3}\right) r_{1} \\
& \xi_{b} \simeq b_{2} r_{4}+b_{3} r_{1}-b_{1} r_{2}+b_{4} r_{2}+b_{1} r_{3} \\
& \xi_{c} \simeq c_{2} r_{4}+c_{4} r_{2}+c_{1}\left(r_{1}-r_{2}+r_{3}\right)
\end{aligned}
$$

vanish for some values of $r_{i}>0$ for which in addition $r_{1}-r_{2}+r_{3}>0$.

\section{A 3-generation GUT model}

As a quick search reveals it is indeed possible to find globally consistent supersymmetric models with 3 chiral generations of SU(5) GUT matter. As one example out of the $\mathrm{O}(100)$ models we found we present the configuration with non-vanishing $B$-field

$$
B=\frac{1}{2} D_{5}+\frac{1}{2} D_{7}
$$

and line bundles

$$
\begin{aligned}
& L_{a}=\iota_{a}^{*} \mathcal{O}_{Y}\left(-\frac{7}{2} D_{5}-2 D_{7}\right), \\
& L_{b}=\iota_{b}^{*} \mathcal{O}_{Y}\left(-D_{5}-D_{6}+\frac{1}{2} D_{7}\right), \\
& L_{c}=\iota_{c}^{*} \mathcal{O}_{Y}\left(-D_{5}+2 D_{7}\right) .
\end{aligned}
$$

According to Table 10 this choice yields precisely 3 chiral GUT families of $\mathbf{1 0}, \overline{\mathbf{5}}$ and $N_{R}^{c}$ with no chiral exotics. In addition there is chiral hidden matter as summarised in Table 11. 


\begin{tabular}{|c||c|c|c|}
\hline \hline chirality & $U(5)$ & $U(1)$ & $U(3)$ \\
\hline \hline 3 & $\Xi_{(2)}$ & 1 & 1 \\
3 & $\square_{(-1)}$ & $\bar{\square}_{(-1)}$ & 1 \\
3 & 1 & $\square(2)$ & 1 \\
\hline \hline 1 & 1 & $\square_{(-1)}$ & $\square(1)$ \\
1 & 1 & $\square(1)$ & $\square(1)$ \\
2 & 1 & 1 & $\square_{(2)}$ \\
\hline
\end{tabular}

Table 11: Chiral spectrum for intersecting D7-brane model with indices denoting the $U(1)$ charges. The last three lines are completely hidden chiral matter.

The contributions to the D3-brane tadpole of this GUT model is

$$
N_{\text {gauge }}=-\frac{5}{2} \cdot \frac{9}{4}+\frac{1}{2} \cdot \frac{17}{4}+\frac{3}{2} \cdot \frac{4}{4}=-2
$$

so that at this stage we would need to add $N_{D 3}=12$ dynamical D3-branes. Note that it is suspicious that the contribution of the gauge flux on the $S U(5)$ brane to the D3-brane tadpole is negative. Indeed, we will see in a moment that this bundle leads to ghosts. We will avoid this conclusion by twisting it by an additional bundle $R_{a}$ which is trivial on $Y$.

For the above choice of B-field the K-theory constraints from $D_{5}, D_{6}, D_{8}$ are vacuous. As expected from the general consideration in Subsection 4.6, the D-term constraint for $D_{a}$ drives us to the boundary of Kähler moduli space in that it requires $r_{1}=0$. The general solution of the three D-term equations for the Kähler moduli is

$$
r_{1}=0, \quad r_{2}=x, \quad r_{3}=x, \quad r_{4}=0 .
$$

For positive $x$ this solution lies on the boundary of Kähler moduli space in that besides $r_{1}=0$ and $r_{4}=0$ also the volume of the generator $C^{5}$ of the Mori cone vanishes. In this regime the classical volume of the divisor $D_{7}$ vanishes, while all other brane volumes and the total volume of the Calabi-Yau are positive. Note that for this model the classical value of the GUT gauge coupling is $\alpha_{G U T}^{-1} \simeq-c_{1}^{2}\left(L_{a}\right)<0$, which we have just seen to be negative. This is another indication that the model 
is pathological in its present form and will be rectified momentarily by twisting $L_{a}$ further.

\section{GUT breaking and $\mathrm{SU}(3) \times \mathrm{SU}(2) \times \mathrm{U}(1)$ spectrum}

To break the GUT symmetry to $S U(3) \times S U(2) \times U(1)_{Y}$ and to compute the vectorlike MSSM spectrum we need the explicit pushforward and pullback maps $\iota_{*}$ and $\iota^{*}$ between the second (co)homology of $D_{7}$ and the ambient space $Y$. Recall that $D_{7}$ is a $\mathrm{dP}_{8}$ surface with $H_{2}\left(D_{7}, \mathbb{Z}\right)$ spanned by $h, e_{1}, \ldots e_{8}$. The pushforward $\iota_{*}$ : $H_{2}\left(D_{7}, \mathbb{Z}\right) \rightarrow H_{2}(Y, \mathbb{Z})$ follows immediately once one takes into account that relative to $\pi^{*}\left(E_{1}\right)=\mathrm{dP}_{9}$ the curve $\mathcal{B} E_{1}$ is flopped away in the present phase. Explicitly,

$$
\iota_{*}\left(e_{i}\right)=f, \quad i=1, \ldots 8, \quad \iota_{*}(h)=3 f,
$$

where $-f \in H_{2}(Y, \mathbb{Z})$ now denotes the class of the curve $D_{7} \cap D_{7}$ in $Y$. This in turn follows from $\iota^{*} D_{7}=K_{D_{7}}=-3 h+\sum e_{i}$ together with the identity $\iota_{*} \iota^{*}=1$. Finally one completes the pullback map to

$$
\iota^{*} D_{7}=-3 h+\sum_{i=1}^{8} e_{i}, \quad \iota^{*} D_{5}=3 h-\sum_{i=1}^{8} e_{i}
$$

and all others vanishing. Therefore, one finds for the pullback of $c_{1}\left(L_{a}\right)$ to $D_{7}$

$$
\mathrm{c}_{1}\left(L_{a}\right)=-\frac{3}{2}\left(3 h-\sum_{i=1}^{8} e_{i}\right) .
$$

For this bundle we can now compute

$$
H^{*}\left(\mathrm{dP}_{8}, L_{a}^{\vee} \otimes L_{a}^{\vee}\right)=(7,0,0), \quad H^{*}\left(\mathrm{dP}_{8}, L_{a} \otimes L_{a}\right)=(0,0,4),
$$

which implies $\operatorname{Ext}^{*}\left(\iota_{*} L_{a}^{\vee}, \iota_{*} L_{a}\right)=(7,4,0,0)$. Therefore, this line bundle on the $S U(5)$ stack leads to ghosts in the spectrum. Since the Kähler form is on the boundary of the Kähler cone this is not in contradiction with the no-ghost theorem from Subsection 2.4. However, we still have the freedom to tensor $L_{a}$ with a line bundle $R_{a}$ which is trivial on the ambient space $Y$. This does not change the chiral spectrum but the non-chiral one.

This freedom can be used to choose the bundles $\mathcal{L}_{a}=L_{a} \otimes R_{a}$ and $\mathcal{L}_{Y}$ as

$$
\mathrm{c}_{1}\left(\mathcal{L}_{a}\right)=\frac{1}{2}\left(-h+\sum_{i=1}^{4} e_{i}-\sum_{i=5}^{8} e_{i}\right), \quad \mathrm{c}_{1}\left(\mathcal{L}_{Y}\right)=-e_{1}+e_{5} .
$$


This configuration leads to the multiplicities displayed in Table 12 for the decomposition of the 10 into MSSM states. For its computation see Appendix B. Note the appearance of only two extra vector-like states.

\begin{tabular}{|c||c|c|}
\hline \hline repr. & extension & spectrum \\
\hline \hline$(\overline{\mathbf{3}}, \mathbf{1})_{-4_{Y}}$ & $\operatorname{Ext}^{*}\left(\mathcal{L}_{a}^{\vee}, \mathcal{L}_{a}\right)$ & $(0,1,4,0)$ \\
\hline$(\mathbf{3}, \mathbf{2})_{1_{Y}}$ & $\operatorname{Ext}^{*}\left(\mathcal{L}_{a}^{\vee} \otimes \mathcal{L}_{Y}^{-1}, \mathcal{L}_{a}\right)$ & $(0,0,3,0)$ \\
\hline$(\mathbf{1}, \mathbf{1})_{6_{Y}}$ & $\operatorname{Ext}^{*}\left(\mathcal{L}_{a}^{\vee} \otimes \mathcal{L}_{Y}^{-2}, \mathcal{L}_{a}\right)$ & $(0,1,4,0)$ \\
\hline \hline
\end{tabular}

Table 12: Multiplicities of MSSM descendants from the SU(5) 10.

To compute the MSSM descendants of the $\overline{\mathbf{5}}$ we recall that the divisors $D_{7}$ and $D_{5}$ intersect again along a genus 1 curve $F$ with $c_{1}\left(\left.\mathcal{L}_{Y}\right|_{F}\right)=0$. We will make use of our freedom to twist also $L_{b}$ by a line bundle $R_{b}$ which is trivial on the Calabi-Yau $\mathrm{Y}$ and define

$$
\mathcal{L}_{b}=L_{b} \otimes R_{b}
$$

Since $R_{b}$ is trivial on $Y$ this does not change the chiral spectrum. Irrespective of its form we find $c_{1}\left(\left.\mathcal{L}_{a}^{\vee} \otimes \mathcal{L}_{b}^{\vee}\right|_{D_{5} \cap D_{7}}\right)=3$, leading to precisely 3 multiplets of $(\overline{\mathbf{3}}, \mathbf{1})_{2 Y}$ and $(\mathbf{1}, \mathbf{2})_{-3 Y}$ and no extra vector-like states.

On the other hand we will choose $R_{b}$ such that the Wilson lines for the bundles $\left.\mathcal{L}_{a}^{\vee} \otimes \mathcal{L}_{b}\right|_{D_{5} \cap D_{7}}$ and $\left.\mathcal{L}_{a}^{\vee} \otimes \mathcal{L}_{Y}^{\vee} \otimes \mathcal{L}_{b}\right|_{D_{5} \cap D_{7}}$ give rise to precisely one pair of Higgs doublets and no Higgs triplet. To this end we recall that $D_{5}$ is a $\mathbb{P}^{2}$ surface with 11 points blown up to a $\mathbb{P}^{1}$, and in analogy with the notation for del Pezzo surfaces $H^{2}\left(D_{5}, \mathbb{Z}\right)$ is spanned by $h, e_{1}, \ldots e_{9}, E_{10}, E_{11}$. Here $E_{10}$ and $E_{11}$ denote the extra two $\mathbb{P}^{1}$ which have been flopped into $D_{5}$ in the present phase. For a special choice of complex structure moduli the elliptic curve $D_{5} \cap D_{7}$ intersects $h, e_{1}, \ldots e_{8}$ in the same points as the classes $24, e_{1}, \ldots e_{8}$ in $H^{2}\left(D_{7}\right)$. It is then clear that, given the choice eq. (189) for $\mathcal{L}_{a}$ and $\mathcal{L}_{Y}$, we have to pick

$$
R_{b}=\mathcal{O}\left(4 h-2 e_{1}-e_{2}-e_{3}-e_{4}-e_{5}-2 e_{6}-2 e_{7}-2 e_{8}\right)
$$

\footnotetext{
${ }^{24}$ This is actually more than we need since we only have to ensure that the sum of the Wilson lines add up to zero to engineer one Higgs pair.
} 
on $D_{5}$ to comply with the requirement stated in equation (65).

The twist with the bundles $R_{a}, R_{b}$ and the addition of the bundle $\mathcal{L}_{Y}$ change the overall contributions of the gauge fluxes to the D3-brane tadpole. These now read

$$
N_{\text {gauge }}=\frac{5}{2} \cdot \frac{7}{4}+\frac{1}{2} \cdot\left(\frac{17}{4}+4\right)+\frac{3}{2}=10
$$

This time $N_{\text {gauge }}$ precisely equals the D3-brane charge of the orientifold planes, and cancellation of the full D3-brane tadpole is possible in a supersymmetric manner without introducing further $D 3$-branes. Moreover, note that despite the vanishing volume of the GUT divisor $D_{7}=\mathrm{dP}_{8}$, the gauge coupling now comes out positive.

Finally, one might wonder about the existence of ghosts on the $U(1)$ and $U(3)$ branes since we have not been able to satisfy all three D-term supersymmetry conditions inside the Kähler cone. On the other hand, one can convince oneself that it is possible to satisfy the supersymmetry conditions inside the Kähler cone for each of the line bundles $L_{b}$ and $L_{c}$ separately. This is already enough for our lemma in Subsection 2.4 to guarantee absence of states in $\mathrm{Ext}^{0}$ and $\mathrm{Ext}^{3}$.

\section{Instanton effects}

The existence of a model with three chiral generations of MSSM matter rested upon the choice eq. (181) for the B-field. The downside of this choice is that none of the invariant divisors $D_{5}, D_{6}, D_{8}$ allows for trivial line bundles. As a consequence there exist no divisors that would give rise to symplectic gauge groups for spacetime-filling branes, and thus no $O(1)$ instantons. To decide if neutrino Majorana masses or the $10105_{\mathbf{H}}$ coupling are generated non-perturbatively we would therefore have to study the effects of D3-brane instantons wrapping non-invariant cycles, for example along the lines of [78]. This, however, is beyond the scope of the present work.

We conclude this section by summarising the key phenomenological properties of our model in Table 13,

\subsection{A GUT Model on $\mathrm{M}_{3}^{\left(\mathrm{dP}_{9}\right)^{3}}$}

In this section we investigate whether we can build a GUT model, where the $\mathbf{1 0}$ representation is also localised on a curve. This is expected to avoid the appearance of extra vector-like states.

Concretely, we consider the elliptic fibration over the $\mathrm{dP}_{3}$ base in the Weierstraß phase with the section $\mathcal{B}=\mathrm{dP}_{3}$ and the six $\mathrm{dP}_{9}$ pull-back divisors $\pi^{*}\left(E_{1}\right), \pi^{*}\left(E_{2}\right)$, 


\begin{tabular}{|c|c|c|}
\hline property & mechanism & status \\
\hline globally consistent & tadpoles + K-theory & $\checkmark^{*}$ \\
\hline D-term susy & vanishing FI-terms inside Kähler cone & $\checkmark^{* *}$ \\
\hline gauge group $S U(5)$ & $U(5) \times U(1)$ stacks & $\checkmark$ \\
\hline 3 chiral generations & choice of line bundles & $\checkmark$ \\
\hline no vector-like matter & localisation on $\mathbb{P}^{1}$ curves & - \\
\hline 1 vector-like of Higgs & choice of line bundles & $\checkmark^{* * *}$ \\
\hline no adjoints & rigid 4-cycles, del Pezzo & $\checkmark$ \\
\hline GUT breaking & $U(1)_{Y}$ flux on trivial 2-cycles & $\checkmark$ \\
\hline 3-2 splitting & Wilson lines on $g=1$ curve & $\checkmark$ \\
\hline $3-2$ split + no dim $=5$ p-decay & local. of $H_{u}, H_{d}$ on disjoint comp. & - \\
\hline $10 \overline{5} \overline{5}_{\mathrm{H}}$ Yukawa & perturbative & $\checkmark$ \\
\hline $10105_{\mathbf{H}}$ Yukawa & presence of appropriate D3-instanton & $--^{* * * *}$ \\
\hline Majorana neutrino masses & presence of appropriate D3-instanton & $--^{* * * *}$ \\
\hline
\end{tabular}

Table 13: Summary of $S U(5)$ properties realised in the model of Subsection 6.1.

* K-theory to the best of our ability to detect SP cycles

** realised on acceptable boundary of Kähler cone

*** for special choice of complex structure moduli

**** at least not with $O(1)$ instantons 
$\pi^{*}\left(E_{3}\right), \pi^{*}\left(l-E_{1}-E_{2}\right), \pi^{*}\left(l-E_{1}-E_{3}\right)$ and $\pi^{*}\left(l-E_{2}-E_{3}\right)$. Moreover, we choose the involution acting as

$$
\left(\begin{array}{c}
l \\
E_{1} \\
E_{2} \\
E_{3}
\end{array}\right) \mapsto\left(\begin{array}{c}
2 l-E_{1}-E_{2}-E_{3} \\
l-E_{1}-E_{3} \\
l-E_{2}-E_{3} \\
l-E_{1}-E_{2}
\end{array}\right)
$$

on the $\mathrm{dP}_{3}$. The orientifold $O 7$-plane wraps the divisor

$$
D_{O 7}=\pi^{*}\left(2 l-E_{1}-E_{2}\right)
$$

which has $\chi\left(D_{O 7}\right)=48$. Since there are no fixed points for this involution, there are no O3-planes. and the contribution of the curvature terms to the D3 tadpole condition is $N_{D 3}+N_{\text {flux }}=12$.

\section{Kähler cone}

Expanding the Kähler form as

$$
J=r_{\mathcal{B}} \mathcal{B}+r_{l} \pi^{*}(l)-r_{1} \pi^{*}\left(E_{1}\right)-r_{2} \pi^{*}\left(E_{2}\right)-r_{3} \pi^{*}\left(E_{3}\right) .
$$

the Kähler cone is simply

$$
r_{\mathcal{B}}>0, \quad r_{i}-r_{\mathcal{B}}>0, \quad r_{l}-r_{i}-r_{j}-r_{\mathcal{B}}>0, \quad i<j \in\{1,2,3\} .
$$

However, the involution $\sigma$ has $h_{-}^{1,1}=2$, so that we expect that two of these five Kähler moduli are fixed. Indeed, requiring that $J$ is invariant under $\sigma$ yields the two relations

$$
r_{1}=r_{2}, \quad r_{l}=2 r_{2}+r_{3}
$$

We are only left with three dynamical Kähler moduli.

In addition, in this case we have two $B_{-}$moduli. With the general Ansatz

$$
B_{-}=b_{\mathcal{B}} \mathcal{B}+b_{l} \pi^{*}(l)-b_{1} \pi^{*}\left(E_{1}\right)-b_{2} \pi^{*}\left(E_{2}\right)-b_{3} \pi^{*}\left(E_{3}\right)
$$

subject to $\sigma\left(B_{-}\right)=-B_{-}$we obtain the three constraints

$$
b_{\mathcal{B}}=0, \quad b_{l}=b_{3} \quad b_{1}=2 b_{3}-b_{2} .
$$




\section{Tadpole cancellation}

To cancel the D7-brane tadpole eq. (13) we introduce three stacks of D7-branes on the divisors

$$
\begin{aligned}
& D_{a}=\pi^{*}\left(E_{2}\right), \quad D_{a}^{\prime}=\pi^{*}\left(l-E_{2}-E_{3}\right), \\
& D_{b}=\pi^{*}\left(l-E_{1}\right), \quad D_{b}^{\prime}=\pi^{*}\left(l-E_{2}\right), \\
& D_{c}=\pi^{*}\left(E_{3}\right), \quad D_{c}^{\prime}=\pi^{*}\left(l-E_{1}-E_{2}\right) \text {. }
\end{aligned}
$$

As for the line bundles it is convenient to split off the continuous $B_{-}$-moduli by writing $c_{1}(\widetilde{L})=c_{1}(L)-B_{-}$. Choosing the $\mathcal{B}$ part of the line bundles on these divisors as

$c_{1}\left(\widetilde{L}_{a}\right)=3 k \mathcal{B}+\pi^{*}\left(\eta_{a}\right), \quad c_{1}\left(\widetilde{L}_{b}\right)=-5 k \mathcal{B}+\pi^{*}\left(\eta_{b}\right), \quad c_{1}\left(\widetilde{L}_{c}\right)=-3 k \mathcal{B}+\pi^{*}\left(\eta_{c}\right)$,

cancels also the D5-brane tadpole. The resulting chiral spectrum is listed in Table 14.

\begin{tabular}{|c||c|c|c|}
\hline \hline number & $U(5)$ & $U(3)$ & $U(5)$ \\
\hline \hline $6 k$ & $\square_{(2)}$ & 1 & 1 \\
$2 k$ & $\bar{\square}_{(-1)}$ & $\bar{\square}_{(-1)}$ & 1 \\
$10 k$ & 1 & $\bar{\Xi}_{(-2)}$ & 1 \\
\hline \hline
\end{tabular}

Table 14: Chiral spectrum for intersecting D\%-brane model. The indices denote the $U(1)$ charges.

Some remarks are in order concerning this spectrum: One gets precisely $6 k$ generations of $\overline{\mathbf{1}} \mathbf{0}$ and, taking into account the extra multiplicities due to the $U(3)$ stack, $2 k \times 3$ generations of $\overline{\mathbf{5}}$, but without right-handed neutrinos. In fact we found that none of the pull-back divisors carries symplectic Chan-Paton factors. In this model the flavour group is gauged. Moreover, since $E_{2}$ and $l-E_{1}$ do not intersect there are no massless Higgs fields in the $(a b)$ sector. However in the $\left(a c^{\prime}\right)$ sector, vanishing Wilson-lines along the elliptic fibre imply that we obtain one vector-like matter field in the $(\mathbf{5}, \mathbf{1}, \mathbf{5})+(\overline{\mathbf{5}}, \mathbf{1}, \overline{\mathbf{5}})$ representation. These carry the GUT quantum numbers to be identified with five pairs of Higgs fields. As mentioned in Subsection 3.1, 
since the Higgs and $\overline{\mathbf{5}}$ matter fields are charged under different U(1) groups also the bottom-type Yukawa couplings need to be realised by D3-brane instantons. Clearly, this is not a completely realistic model, but some rough features are realised and in particular the massless matter states in the anti-symmetric representation of $S U(5)$ are localised on the fibre $F$ of the elliptic fibration. The minimal choice $k=\frac{1}{2}$ gives already three generations.

\section{Three generation model}

Taking now the quantisation conditions for the gauge fluxes into account and turning on half-integer $B$-field flux through the fibre, that is, $c_{1}(B)=\frac{1}{2} \mathcal{B}$, we choose

$$
\begin{aligned}
c_{1}\left(\widetilde{L}_{a}\right) & =\frac{3}{2} \mathcal{B}+\frac{3}{2} \pi^{*}\left(E_{2}\right), \quad c_{1}\left(\widetilde{L}_{b}\right)=-\frac{5}{2} \mathcal{B}+5 \pi^{*}\left(E_{1}\right), \\
c_{1}\left(\widetilde{L}_{c}\right) & =-\frac{3}{2} \mathcal{B}-\frac{3}{2} \pi^{*}\left(E_{3}\right)
\end{aligned}
$$

for the line bundles.

The D-term constraints

$$
\int_{D_{a}} J \wedge\left(c_{1}\left(\widetilde{L}_{a}\right)+B_{-}\right)=0
$$

for all three brane stacks give three constraints which can all be solved inside the Kähler cone provided

$$
r_{\mathcal{B}}>0 \quad-\frac{3}{2}<b_{2}<\frac{3}{2}
$$

The contributions of each of the three stacks to the D3-tadpole condition are positive and add up as

$$
N_{\text {gauge }}=\frac{3 \cdot 45}{8}+\frac{3 \cdot 75}{4}+\frac{3 \cdot 45}{8}=90 \gg 12 .
$$

Here we see explicitly that eventually the $B_{-}$field drops out so that one ends up really with an integer contribution. Clearly this is a massive overshooting and requires the introduction of anti D3-branes. Finally, for $c_{1}(B)=\frac{1}{2} \mathcal{B}$ the divisor $\mathcal{B}$ can carry a trivial line bundle and is expected to have $S P$ Chan-Paton factors. The resulting global Witten anomaly (K-theory) constraint is satisfied. 


\section{Non-chiral spectrum and GUT breaking}

Nevertheless, the purpose was to demonstrate that by choosing non-diagonal involutions, it is (in principle) possible to have also the matter in the antisymmetric representation of $S U(5)$ localised on a curve. In fact here all matter is localised in the fibre elliptic curve $C=F$ with trivial canonical line-bundle. From that it immediately follows that there is no vector-like matter in the $\mathbf{1 0}+\overline{\mathbf{5}}$ representation. Since the $U(5)$ stack is a rigid $\mathrm{dP}_{9}$ surface, we can break the $S U(5)$ GUT gauge symmetry to the Standard Model gauge group by turning on $U(1)_{Y}$ flux of the form $\mathcal{L}_{Y}=\mathcal{O}\left(e_{1}-e_{2}\right)$. In contrast to the first two examples we presented, this does not give any new vector-like matter. As for the Higgs sector, we are now in the favourable situation that the bundles $\tilde{L}_{a}$ and $\tilde{L}_{b}$ are both pullbacks from the ambient space. According to the discussion around eq. (66) it therefore suffices to twist $\tilde{L}_{a}$ by $R_{a}=\mathcal{L}_{Y}^{-1}$ to arrange for precisely one Higgs doublet and no Higgs triplet without further adjusting any complex structure moduli. Adding $\mathcal{L}_{Y}$ and $R_{a}$ results in an additional 3 units of D3-brane charge in the D3-tadpole equation.

\section{Summary of features}

Let us summarise in Table 15 which of the desired features we were able to realise in this simple model.

\section{GUTs on Del Pezzo Transitions of the Quintic}

So far we have studied GUT models on descendants of the elliptic fibration CalabiYau $\mathbb{P}_{1,1,1,6,9}[18]$. We have gone a long way to eventually arrive at GUT like examples featuring many of the desired properties. One of the general aspects of these models was that for the $S U(5)$ GUT stack localised on a shrinkable $\mathrm{dP}_{8}$ surface the D-term conditions in conjunction with the swiss-cheese property of the triple intersection form force the GUT four-cycle to collapse to string scale size, that is, to the quiver locus. If this were a generic feature of all Calabi-Yau orientifolds containing shrinkable surfaces, it would clearly have strong implications for model building.

The clarification of this point is one of our motivations for studying another class of Calabi-Yau manifolds containing del Pezzo surfaces. Instead of starting with the elliptic fibration $\mathbb{P}_{1,1,1,6,9}[18]$, we take the simple Quintic $\mathbb{P}_{1,1,1,1,1}[5]$ and perform del Pezzo transitions. The mathematics of this construction is collected in Subsection 7.1 


\begin{tabular}{|c||c|c|}
\hline \hline property & mechanism & status \\
\hline \hline globally consistent & tadpoles + K-theory & $\checkmark^{*, * *}$ \\
D-term susy & vanishing FI-terms inside Kähler cone & $\checkmark$ \\
gauge group $S U(5)$ & $U(5) \times U(3)$ stacks & $\checkmark$ \\
3 chiral generations & choice of line bundles & $\checkmark$ \\
no vector-like matter & localisation on g=1 curves & $\checkmark$ \\
5 vector-like Higgs & choice of line bundles & $\checkmark$ \\
no adjoints & rigid 4-cycles, del Pezzo & $\checkmark$ \\
GUT breaking & W(1) $)_{Y}$ flux on trivial 2-cycles & $\checkmark$ \\
$3-2$ splitting & Wilson lines on $g=1$ curve & $\checkmark$ \\
$3-2$ split + no dim=5 p-decay & local. of $H_{u}, H_{d}$ on disjoint comp. & - \\
$\mathbf{1 0} \overline{\mathbf{5}} \overline{\mathbf{5}}_{\mathbf{H}}$ Yukawa & presence of appropriate D3-instanton & $-{ }^{* * *}$ \\
$\mathbf{1 0} \mathbf{1 0} \boldsymbol{5}_{\mathbf{H}}$ Yukawa & presence of appropriate D3-instanton & $-{ }^{* * *}$ \\
Majorana neutrino masses & presence of appropriate D3-instanton & $-{ }^{* * *}$ \\
\hline \hline
\end{tabular}

Table 15: Summary of $S U(5)$ properties realised in the model of Subsection 6.2.

* overshooting in D3-tadpole $\rightarrow \bar{D} 3$-branes

** K-theory to the best of our ability to detect $S P$ cycles

*** at least not with $O(1)$ instantons 
with the result that here we can get intersecting $\mathrm{dP}_{r}, r \leq 8$ surfaces, so that the triple intersection forms do not have the diagonal swiss-cheese type structure. In Subsection 7.2 we provide one more example of a GUT model, for which all Standard Model matter is really localised on curves of genus zero and one, respectively.

\subsection{Del Pezzo Transitions of the Quintic}

In this section we introduce a class of compact Calabi-Yau manifolds which can be obtained from the quintic hypersurface by performing del Pezzo transitions. Again these spaces will be realised as hypersurfaces in an ambient toric manifold. It will turn out that the del Pezzo surfaces arising after the transitions can intersect and thus are ideal candidates for supporting intersecting D7-branes.

\section{The toric data and intersection forms}

Let us first give the points in the polyhedron for the toric ambient spaces. The hypersurface is then determined to have the anti-canonical class given by the sum of all toric divisors as in Subsection 4.2. The quintic hypersurface has the points $v_{1}^{*}=(-1,0,0,0), v_{2}^{*}=(0,-1,0,0) v_{3}^{*}=(0,0,-1,0), v_{4}^{*}=(0,0,0,-1)$ and $v_{5}^{*}=$ $(1,1,1,1)$. Its Hodge numbers are $h^{1,1}=1$ and $h^{2,1}=101$. By arranging some of the $h^{2,1}$ complex structure deformations in the hypersurface constraint one can generate del Pezzo singularities and blow up del Pezzo surfaces. This process increases $h^{1,1}$ by the number of Kähler moduli of the del Pezzo four-cycles and lowers $h^{2,1}$ since one has to fix a certain number of the complex structure moduli to generate a singularity.

As a first transition we can blow up a $\mathrm{dP}_{6}$ surface by adding the point $v_{6}^{*}=$ $(1,0,0,0)$ to the polyhedron and consider the resulting hypersurface $Q^{\mathrm{dP}_{6}}$. In fact, the new Calabi-Yau manifold has $h^{1,1}=2$ and $h^{2,1}=90$. There is only one triangulation for the ambient toric space. Using the same methods as in Subsection 4.2 one can explicitly check that the divisor $D_{6}$ is a $\mathrm{dP}_{6}$ del Pezzo surface. The intersection form is simply

$$
I_{3}=3 D_{6}^{3}+3 D_{5}^{2} D_{6}-3 D_{6}^{2} D_{5}+2 D_{5}^{3} .
$$

This Calabi-Yau is also of the strong swiss-cheese type, since the intersection form diagonalises for the transformation $\left(\tilde{D}_{5}=D_{6}-D_{5}, \tilde{D}_{6}=D_{6}\right)$. This is also consistent with the fact that the $\mathrm{dP}_{6}$ surface in this manifold is generic which can be inferred from the fact that in this transition $\Delta \chi=24$. For generic transitions, as the ones in 
Subsection 4.2 with Euler numbers eq. (79), one has $\Delta \chi=2 C_{(n)}$, where $C_{(n)}$ is the dual Coxeter number of the exceptional groups associated to $\mathrm{dP}_{n}$.

To generate a second del Pezzo surface we add the point $v_{7}^{*}=(0,1,0,0)$ to the polyhedron. This blows up a point on the $\mathrm{dP}_{6}$ surface $D_{6}$ to a $\mathbb{P}^{1}$ such that $\mathrm{dP}_{6} \rightarrow \mathrm{dP}_{7} \cong D_{6}$ and generates a second $\mathrm{dP}_{7}$ surface $D_{7}$. The ambient toric space has one Calabi-Yau triangulation and the hypersurface, denoted by $Q^{\left(\mathrm{dP}_{7}\right)^{2}}$, has Hodge numbers $h^{1,1}=3$ and $h^{2,1}=79$. One can then check that the two $\mathrm{dP}_{7}$ surfaces $D_{6}, D_{7}$ intersect on a $\mathbb{P}^{1}$. To do that one computes the triple intersection form

$$
I_{3}=2 D_{7}^{3}+2 D_{6}^{3}+2 D_{5}^{2}\left(D_{7}+D_{6}\right)-D_{7}^{2}\left(2 D_{5}+D_{6}\right)-D_{6}^{2}\left(2 D_{5}+D_{7}\right)+D_{5} D_{6} D_{7} .
$$

This intersection form cannot be diagonalised due to the intersection of $D_{6}$ and $D_{7}$. In other words, the two $\mathrm{dP}_{7}$ surfaces are not generic, but share a common $\mathbb{P}^{1}$. This can be also inferred from the fact that $\Delta \chi=2 \times 24$ with respect to the quintic hypersurface. This change is expected for a transition with two generic $\mathrm{dP}_{6}$ surfaces and corresponds to the fact that the $E_{6}$ sublattices on the two $\mathrm{dP}_{7}$ surfaces are still trivial in the Calabi-Yau threefold. For two generic $\mathrm{dP}_{7}$ surfaces one would find $\Delta \chi=2 \times 36$. That only an $E_{6}$ sublattice is trivial in the Calabi-Yau can also be inferred by computing the BPS-instantons as suggested in [41. One finds that the representations of $E_{7}$ appearing for a generic $\mathrm{dP}_{7}$ transition are split into $E_{6}$ representations for our intersecting divisors $D_{6}$ and $D_{7}$.

We can perform a third transition by adding the point $v_{8}^{*}=(0,0,1,0)$ to the polyhedron and denote the corresponding hypersurface by $Q^{\left(\mathrm{dP}_{8}\right)^{3}}$. In this case one additional $\mathbb{P}^{1}$ is blown up on each $\mathrm{dP}_{7}$ surface $D_{6}, D_{7}$ such that $\mathrm{dP}_{7} \rightarrow \mathrm{dP}_{8}$. The two $\mathrm{dP}_{8}$ surfaces $D_{6}, D_{7}$ intersect a new $\mathrm{dP}_{8}$ surface $D_{8}$ in the blown-up $\mathbb{P}^{1}$ curves. The new toric ambient space has one triangulation, and the corresponding Calabi-Yau hypersurface has Hodge numbers $h^{1,1}=4$ and $h^{2,1}=68$. The intersection form is given by

$$
\begin{aligned}
& I_{3}=D_{6}^{3}+D_{7}^{3}+D_{8}^{3}-D_{5}^{3}+D_{5}^{2}\left(D_{6}+D_{7}+D_{8}\right) \\
&-D_{6}^{2}\left(D_{5}+D_{7}+D_{8}\right)-D_{7}^{2}\left(D_{5}+D_{6}\right.\left.+D_{8}\right)-D_{8}^{2}\left(D_{5}+D_{6}+D_{7}\right) \\
&+D_{5}\left(D_{6} D_{8}+D_{7} D_{8}+D_{7} D_{6}\right) .
\end{aligned}
$$

Once again we note that the $\mathrm{dP}_{8}$ surfaces are non-generic, since they intersect over $\mathbb{P}^{1}$ curve. This is an accord with the fact that $\Delta \chi=3 \times 24$ with respect to the quintic. In fact, one concludes that in each del Pezzo 8 surface and $E_{6}$ sublattice is trivial in the Calabi-Yau space. 
Finally, we can add the point $v_{9}^{*}=(0,0,0,1)$ to the polyhedron and denote the corresponding hypersurface by $Q^{\left(\mathrm{dP}_{9}\right)^{4}}$. In fact, the new Calabi-Yau space has $h^{1,1}=5$ and $h^{2,1}=57$ and one checks that the toric divisors $D_{6}, D_{7}, D_{8}, D_{9}$ are $\mathrm{dP}_{9}$ surfaces. Clearly, this space cannot be obtained by resolving a del Pezzo singularity, since $\mathrm{dP}_{9}$ can be only shrunk to a curve and not to a point. Nevertheless it is a viable Calabi-Yau background with intersection form

$$
\begin{aligned}
I_{3}=D_{5}\left(D_{6} D_{8}+D_{8} D_{9}+D_{6} D_{9}+D_{6} D_{7}+D_{7} D_{8}+D_{7} D_{9}\right) \\
-D_{6}^{2}\left(D_{7}+D_{8}+D_{9}\right)-D_{7}^{2}\left(D_{6}+D_{8}+D_{9}\right)-D_{8}^{2}\left(D_{6}+D_{7}+D_{8}\right) \\
-D_{9}^{2}\left(D_{6}+D_{7}+D_{8}\right)-D_{5}^{3} .
\end{aligned}
$$

One can check that each of the $\mathrm{dP}_{9}$ divisors intersects the other three in a $\mathbb{P}^{1}$. Schematically the intersecting del Pezzo surfaces in the four manifolds $Q^{\mathrm{dP}_{6}}, Q^{\left(\mathrm{dP}_{7}\right)^{2}}$, $Q^{\left(\mathrm{dP}_{8}\right)^{3}}$ and $Q^{\left(\mathrm{dP}_{9}^{4}\right)}$ are shown in Figure 3. The Euler number has changed again by 24 , such that $\Delta \chi=4 \times 24$ with respect to the quintic hypersurface. Again, this corresponds to the fact that there are four $E_{6}$ lattices on the $\mathrm{dP}_{9}$ surfaces which are trivial in $Q^{\left(\mathrm{dP}_{9}\right)^{4}}$.

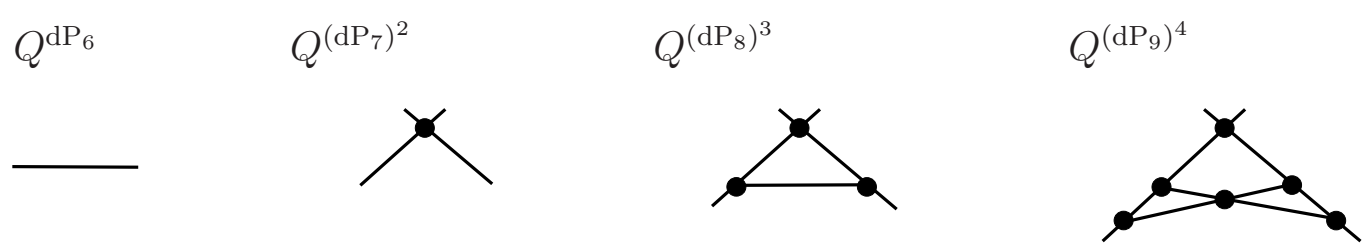

Figure 3: Schematics of the intersecting del Pezzo surfaces on transitions of the quintic. Each intersection is a $\mathbb{P}^{1}$.

\section{Kähler cone and orientifold involution}

In Sections 7.2 and 7.3 we will construct GUT models on $Q^{\left(\mathrm{dP}_{8}\right)^{3}}$ and $Q^{\left(\mathrm{dP}_{9}\right)^{4}}$ with matter and Higgs localised on curves. However, in order to determine the spectrum and check the supersymmetry conditions, we first need to calculate the Kähler cone and the orientifold involution acting on it.

Let us begin by analysing the hypersurface $Q^{\left(\mathrm{dP}_{8}\right)^{3}}$. The Mori cone is simplicial 
for this Calabi-Yau phase, and generated by the vectors

\begin{tabular}{|c|c|c|c|c|c|c|c|c||c|}
\hline & $x_{1}$ & $x_{2}$ & $x_{3}$ & $x_{4}$ & $x_{5}$ & $x_{6}$ & $x_{7}$ & $x_{8}$ & $p$ \\
\hline \hline$\ell^{(1)}$ & 1 & 0 & 0 & 1 & 1 & 0 & -1 & -1 & -1 \\
$\ell^{(2)}$ & 0 & 0 & 0 & -1 & -1 & 1 & 1 & 1 & -1 \\
$\ell^{(3)}$ & 0 & 0 & 1 & 1 & 1 & -1 & -1 & 0 & -1 \\
$\ell^{(4)}$ & 0 & 1 & 0 & 1 & 1 & -1 & 0 & -1 & -1 \\
\hline
\end{tabular}

Its dual, the Kähler cone, is therefore again simplicial and generated by

$$
\begin{array}{ll}
K_{1}=2 D_{5}+D_{6}+D_{7}+D_{8}, & K_{2}=D_{5}+D_{6}, \\
K_{3}=D_{5}+D_{7}, & K_{4}=D_{5}+D_{8} .
\end{array}
$$

As before, the Kähler cone is needed in order to evaluate the D-terms in the physical region of the moduli space.

We next specify a orientifold involution $\sigma$ on $Q^{\left(\mathrm{dP}_{8}\right)^{3}}$. Explicitly, $\sigma$ is given by the exchange of coordinates

$$
\sigma: \quad x_{2} \leftrightarrow x_{3}, \quad x_{7} \leftrightarrow x_{8}
$$

This leads to a split $h_{+}^{1,1}=3$ and $h_{-}^{1,1}=1$, yielding a four-dimensional theory with 3 Kähler moduli $T_{I}$ and one $B_{-}$-modulus $G$ as defined in eq. (11). The fixed point locus of this involution contains one O7-plane wrapping the four-cycle

$$
D_{O 7}=D_{5}+D_{7}+D_{8}
$$

and one fixed point, $N_{O 3}=1$. Note that $\chi\left(D_{O 7}\right)=47$, and, therefore, $\chi\left(D_{O 7}\right)+N_{O 3}$ is indeed divisible by four.

Turning to the other Calabi-Yau phase $Q^{\left(\mathrm{dP}_{9}\right)^{4}}$, the Mori cone is now non-simplicial 
and generated by the vectors

\begin{tabular}{|c|c|c|c|c|c|c|c|c|c||c|}
\hline & $x_{1}$ & $x_{2}$ & $x_{3}$ & $x_{4}$ & $x_{5}$ & $x_{6}$ & $x_{7}$ & $x_{8}$ & $x_{9}$ & $p$ \\
\hline \hline$\ell^{(1)}$ & 0 & 0 & -1 & 0 & -1 & 1 & 1 & 0 & 1 & -1 \\
$\ell^{(2)}$ & 0 & 1 & 0 & 1 & 1 & -1 & 0 & -1 & 0 & -1 \\
$\ell^{(3)}$ & 1 & 0 & 0 & 1 & 1 & 0 & -1 & -1 & 0 & -1 \\
$\ell^{(4)}$ & -1 & 0 & 0 & 0 & -1 & 0 & 1 & 1 & 1 & -1 \\
$\ell^{(5)}$ & 0 & 1 & 1 & 0 & 1 & -1 & 0 & 0 & -1 & -1 \\
$\ell^{(6)}$ & 0 & 0 & 0 & -1 & -1 & 1 & 1 & 1 & 0 & -1 \\
$\ell^{(7)}$ & 0 & 0 & 1 & 1 & 1 & -1 & -1 & 0 & 0 & -1 \\
$\ell^{(8)}$ & 1 & 1 & 0 & 0 & 1 & 0 & 0 & -1 & -1 & -1 \\
$\ell^{(9)}$ & 1 & 0 & 1 & 0 & 1 & 0 & -1 & 0 & -1 & -1 \\
$\ell^{(10)}$ & 0 & -1 & 0 & 0 & -1 & 1 & 0 & 1 & 1 & -1 \\
\hline
\end{tabular}

In order to define coordinates for the Kähler cone, we first discard the $\ell^{(\kappa)}, \kappa=$ $3,4,6,7,8$ and determine the dual basis of four-cycles

$$
\begin{array}{ll}
K_{1}=D_{5}+D_{6}+D_{7}, & K_{2}=D_{5}+D_{8}+D_{9}, \\
K_{3}=D_{5}+D_{6}, & K_{4}=D_{5}+D_{7}+D_{8}, \\
K_{5}=D_{5}+D_{9} . &
\end{array}
$$

Expanding $J=\sum_{i} r_{i} K_{i}$ we have to take $r_{i} \geq 0$ in the Kähler cone. However, we note that the discarded $\ell^{(\kappa)}$ impose the additional conditions

$$
\begin{array}{ll}
r_{3}-r_{4}+r_{5} \geq 0, & r_{2}-r_{3}+r_{4} \geq 0, \\
r_{2}-r_{1}+r_{5} \geq 0, & r_{1}-r_{2}+r_{3} \geq 0 .
\end{array}
$$

We have to ensure that these conditions are satisfied when evaluating the D-terms in coordinates $r_{i}$.

Let us finally specify the involution on $Q^{\left(\mathrm{dP}_{9}\right)^{4}}$. It is simply given by the exchange of $x_{2} \leftrightarrow x_{3}$ and $x_{7} \leftrightarrow x_{8}$, the same as in eq. (212). This leads to a split $h_{+}^{1,1}=4$ and $h_{-}^{1,1}=1$ and, hence, four $T_{I}$ Kähler moduli and one $B_{-}$-modulus $G$. The fixed point locus of this involution contains one O7-plane, wrapping the same linear combination as in eq. (213) and three fixed points, $N_{O 3}=3$. Note that now $\chi\left(D_{O 7}\right)=37$, and, therefore, $\chi\left(D_{O 7}\right)+N_{O 3}$ is again divisible by four. 


\subsection{A GUT Model Without Vector-Like Matter}

In this section we present a $S U(5)$ GUT model with all matter realised on curves. One of our motivations to discuss this example is to illustrate that the GUT brane can indeed wrap a shrinkable $\mathrm{dP}_{r}, r \leq 8$ without being driven to the quiver locus by the D-terms. Our starting point is the Calabi-Yau $Q^{\left(\mathrm{dP}_{8}\right)^{3}}$, which contains three intersecting $\mathrm{dP}_{8}$ surfaces. Using the notation from the previous section, we choose the involution eq. (212), which leads to an O7-plane eq. (213) and one O3-plane. In the following we will specify the D7-branes which define a GUT model with two chiral generations.

\section{Two generation model}

To cancel the D7-brane tadpole (13) we introduce three stacks of D7-branes on the divisors

$$
\begin{array}{rlll}
U(5): & D_{a}=D_{7}, & D_{a}^{\prime}=D_{8}, & \chi\left(D_{a}\right)=11, \\
U(1): & D_{b}=D_{5}, & D_{b}^{\prime}=D_{5}, & \chi\left(D_{b}\right)=25, \\
S O(6): & D_{c}=D_{5}+D_{7}, & D_{c}^{\prime}=D_{5}+D_{8}, & \chi\left(D_{c}\right)=36 .
\end{array}
$$

Here we note that $D_{2}=D_{5}+D_{7}$ and $D_{3}=D_{5}+D_{8}$ are toric divisors. Then the contribution of the curvature terms to the D3-brane tadpole cancellation condition reads

$$
\frac{N_{\mathrm{O} 3}}{4}+\frac{\chi\left(D_{\mathrm{O} 7}\right)}{12}+\sum_{a} N_{a} \frac{\chi_{o}\left(D_{a}\right)}{24}=\frac{1}{4}+\frac{47}{12}+\frac{5 \cdot 11+1 \cdot 25+3 \cdot 36}{24}=12 .
$$

Next, we choose $c_{1}(B)=\frac{1}{2} D_{5}$ and split off the continuous $B_{-}$-moduli by writing $c_{1}(\widetilde{L})=c_{1}(L)-B_{-}$. Taking into account the Freed-Witten quantisation conditions, the following choice of line bundles

$$
c_{1}\left(\widetilde{L}_{a}\right)=\frac{1}{2} D_{5}+\frac{1}{2} D_{7}-D_{8}, \quad c_{1}\left(\widetilde{L}_{b}\right)=D_{5}, \quad c_{1}\left(\widetilde{L}_{c}\right)=0
$$

cancels the D5-brane tadpole as well. Here we used the fact that $D_{7}$ restricts trivially to $D_{c}=D_{5}+D_{7}$. The resulting chiral spectrum is listed in Table 16.

Let us make a couple of remarks concerning this spectrum: One obtains precisely two generations of MSSM particles including the right-handed neutrinos. Moreover, the states transforming in the $\mathbf{1 0}$ representation of $S U(5)$ are localised on the curve $D_{7} \cap D_{8}=\mathbb{P}^{1}$, so that there are no additional vector-like states. Similarly, the 


\begin{tabular}{|c||c|c|c|}
\hline \hline number & $U(5)$ & $U(1)$ & $S O(6)$ \\
\hline \hline 2 & $\square_{(2)}$ & 1 & 1 \\
2 & $\square_{(-1)}$ & $\square_{(1)}$ & 1 \\
2 & 1 & $\bar{\square}_{(-2)}$ & 1 \\
\hline \hline
\end{tabular}

Table 16: Chiral spectrum for intersecting D\%-brane model. The indices denote the $U(1)$ charges.

matter states in the $\overline{\mathbf{5}}$ representation are localised on the curve $D_{7} \cap D_{5}=T^{2}$ so that there are no vector-like states either. Moreover, as in the examples before from the $\left(a^{\prime} b\right)$ sector we will get one vector-like pair of Higgs fields $\mathbf{5}_{H}+\overline{\mathbf{5}}_{H}$ by twisting $L_{b}$ appropriately, see discussion at the end of this subsection. Only the right-handed neutrinos are localised on a surface, namely on $D_{5}$.

A D7-brane wrapped upon $D_{5}$ can carry a trivial line bundle so that this brane is expected to carry symplectic Chan-Paton factors. The resulting K-theory constraint

$$
\int_{Y}\left[D_{5}\right] \wedge\left[D_{7}\right] \wedge c_{1}\left(L_{a}\right)+\int_{Y}\left[D_{7}\right] \wedge\left[D_{5}\right] \wedge c_{1}\left(L_{b}\right) \in 2 \mathbb{Z}
$$

is indeed satisfied for our model. The D3-tadpoles induced by each single brane stack are positive and add up as

$$
N_{\text {gauge }}=\frac{5 \cdot 1}{2}+\frac{1 \cdot 1}{2}=3 .
$$

Let us next evaluate the D-term constraints. The generators $K_{i}$ of the Kähler cone are given in (211). We use these to expand the Kähler form as $J=\sum_{i} r_{i} K_{i}$ with $r_{i}>0$. Note that due to the orientifold action for the last two Kähler cone generators we have $r_{3}=r_{4}$. In addition there exists one $B_{-}$modulus

$$
B_{-}=b\left(D_{7}-D_{8}\right)
$$

The D-term constraints

$$
\int_{D_{i}} J \wedge\left(c_{1}\left(\widetilde{L}_{i}\right)+B_{-}\right)=0
$$


for all three brane stacks $i=a, b, c$ yield three conditions, which are solved on the boundary of the Kähler cone by

$$
r_{1}=0, \quad r_{2}=0, \quad b=0
$$

However, the volumes of the three branes involved and the overall volume are finite

$$
\begin{aligned}
& \tau_{7}=\frac{1}{2} \int_{D_{7}} J \wedge J=r_{3}^{2}, \quad \tau_{5}=\frac{1}{2} \int_{D_{5}} J \wedge J=2 r_{3}^{2}, \\
& \operatorname{Vol}(Y)=\frac{1}{6} \int_{Y} J \wedge J \wedge J=2 r_{3}^{3},
\end{aligned}
$$

so that we still have parametric control over the $\alpha^{\prime}$ expansion in the brane sector.

Finally, to break the $S U(5)$ GUT group to the MSSM, we turn on the trivial line bundle $\mathcal{L}_{Y}$. On $D_{a}=D_{7}=\mathrm{dP}_{8}$ there are now three non-trivial two-cycles. They include the two genus zero curves $\mathbb{P}^{1}$ s from the intersection $D_{7} \cap D_{6}$ and $D_{7} \cap D_{8}$. In addition there exists the genus one curve $D_{7} \cap D_{5}$ which is identical to $-D_{7} \cap D_{7}$. Identifying $D_{7} \cap D_{6}=e_{7}$ and $D_{7} \cap D_{6}=e_{8}$ and $D_{7} \cap D_{5}=3 h-\sum_{i=1}^{8} e_{i}$, we realise that the two-cycles on $\mathrm{dP}_{8}$ trivial in $Y$ are the ones from $\mathrm{dP}_{6}$ orthogonal to $K$. By definition this is the $E_{6}$ sublattice of $H_{2}\left(\mathrm{dP}_{6}, \mathbb{Z}\right)$. Therefore, choosing for instance $c_{1}\left(\mathcal{L}_{Y}\right)=e_{1}-e_{2}$ breaks the $S U(5)$ gauge group to the Standard Model gauge group and contributes additional two units to the D3-brane tadpole. To generate one pair of Higgs doublets and project out the triplet on the elliptic curve $C=D_{5} \cap D_{7}$ we twist $\widetilde{L}_{a}$ by the bundle $R_{a}=\mathcal{L}_{Y}^{-1}$ on $D_{7}$. In essence this yields yet another unit of D3-brane charge in the D3-tadpole equation and we need six dynamical D3-branes to saturate it. Since $\mathcal{L}_{Y}$ restricts trivially to $e_{7}$ and $e_{8}$, there are precisely two generations of charged quark and leptons without any vector like states.

As already mentioned a D7-brane on $D_{5}$ carries $S P$ Chan-Paton factors, so that an Euclidean D3-brane instanton on the same cycle is of type $O(1)$. Indeed, such an instanton carries the right chiral zero modes $I_{a \text {,inst }}=1$ and $I_{b \text {,inst }}=1$ to generate the top-Yukawa couplings. However, since the surface $D_{5}$ has $h^{(2,0)}\left(D_{5}\right)=1$, it is not rigid and there can be additional vector-like zero modes from the intersection of the instanton with the D7-brane wrapping $D_{b}=D_{5}$.

We summarise in Table 17 which of the desired features we were able to realise in this simple model.

\footnotetext{
${ }^{25}$ There is a change of sign compared to (45) as here the Higgs originates from the sector $\left(a^{\prime} b\right)$ instead of $(a b)$.
} 


\begin{tabular}{|c||c|c|}
\hline \hline property & mechanism & status \\
\hline \hline globally consistent & tadpoles + K-theory & $\checkmark^{*}$ \\
D-term susy & vanishing FI-terms inside Kähler cone & $\checkmark^{* *}$ \\
gauge group $S U(5)$ & $U(5) \times U(1)$ stacks & $\checkmark$ \\
3 chiral generations & choice of line bundles & - \\
no vector-like matter & localisation on $g=0,1$ curves & $\checkmark$ \\
5 vector-like Higgs & choice of line bundles & $\checkmark$ \\
no adjoints & rigid 4-cycles, del Pezzo & $\checkmark$ \\
GUT breaking & $U(1)_{Y}$ flux on trivial 2-cycles & $\checkmark$ \\
$3-2$ splitting & Wilson lines on $g=1$ curve & $\checkmark$ \\
3 split + no dim=5 p-decay & local. of $H_{u}, H_{d}$ on disjoint comp. & - \\
$\mathbf{1 0} \overline{\mathbf{5}} \overline{\mathbf{5}}_{\mathbf{H}}$ Yukawa & perturbative & $\checkmark$ \\
$\mathbf{1 0} \mathbf{1 0} \boldsymbol{5}_{\mathbf{H}}$ Yukawa & presence of appropriate D3-instanton & $\checkmark^{* * *}$ \\
Majorana neutrino masses & presence of appropriate D3-instanton & $-{ }^{* * * *}$ \\
\hline \hline
\end{tabular}

Table 17: SU(5) properties realised in the model of Subsection 7.2.

* K-theory to the best of our ability to detect SP-cycles

** realised on acceptable boundary of Kähler moduli space

*** up to absorption of additional vector-like zero modes

**** at least not with $O(1)$ instantons 


\subsection{A Three-Generation Model With Localised Matter on $\mathrm{Q}^{(\mathrm{dP})^{4}}$}

While in on $Q^{\left(\mathrm{dP}_{8}\right)^{3}}$ global consistency conditions are in conflict with the construction of three-generation models, on its cousin $Q^{\left(\mathrm{dP}_{9}\right)^{4}}$ it turns out possible to find GUT models with three generations and all GUT matter realised on matter curves, but without running into half-integer D3 tadpoles. The construction of these models is almost identical to the two-generation example of the previous subsection, and we can be quite brief.

We again cancel the D7-brane tadpole (13) by introducing three stacks of D7branes on the divisors

$$
\begin{array}{llll}
U(5): & D_{a}=D_{7}, & D_{a}^{\prime}=D_{8}, & \chi\left(D_{7}\right)=12, \\
U(1): & D_{b}=D_{5}, & D_{b}^{\prime}=D_{5}, & \chi\left(D_{5}\right)=13, \\
U(3): & D_{c},=D_{5}+D_{7}, & D_{c}^{\prime}=D_{5}+D_{8} & \chi\left(D_{5}+D_{7}\right)=25 .
\end{array}
$$

We consider the non-trivial $B_{+}$-flux

$$
c_{1}(B)=\frac{1}{2}\left(D_{7}+D_{8}+D_{9}\right)
$$

which allows us to introduce the well-defined bundles

$$
\begin{aligned}
& c_{1}\left(\widetilde{L}_{a}\right)=3 D_{5}+2 D_{7}-\frac{1}{2} D_{8}+\frac{1}{2} D_{9}, \\
& c_{1}\left(\widetilde{L}_{b}\right)=\frac{5}{2} D_{5}+D_{6}-\frac{1}{2} D_{7}+\frac{5}{2} D_{8}-\frac{1}{2} D_{9}, \\
& c_{1}\left(\widetilde{L}_{c}\right)=\frac{1}{2} D_{5}+\frac{3}{2} D_{8}-\frac{1}{2} D_{9} .
\end{aligned}
$$

This configuration cancels the D5-tadpole and achieves a pure three-generation spectrum as summarised in Table 18. All phenomenological considerations detailed in the previous section regarding the spectrum of this model apply also for the present three-generation model since the intersection pattern of the divisors $D_{a}, D_{b}$ and $D_{c}$ is unchanged.

The only differences occur when analysing the global consistency and supersymmetry conditions, as we next discuss. Prior to breaking $S U(5)$ via $U(1)_{Y}$ flux the D3-brane tadpole

$$
N_{D 3}+N_{\text {gauge }}=10
$$




\begin{tabular}{|c||c|c|c|}
\hline \hline number & $U(5)$ & $U(1)$ & $U(3)$ \\
\hline \hline 3 & $\boxminus_{(2)}$ & 1 & 1 \\
3 & $\bar{\square}_{(-1)}$ & $\square_{(1)}$ & 1 \\
3 & 1 & $\bar{\square}_{(-2)}$ & 1 \\
\hline 2 & 1 & 1 & $\bar{\square}_{(-2)}$ \\
2 & 1 & $\bar{\square}_{(-1)}$ & $\bar{\square}_{(-1)}$ \\
\hline \hline
\end{tabular}

Table 18: Chiral spectrum for intersecting D\%-brane model. The indices denote the $U(1)$ charges.

is just satisfied without room for extra $D 3$-branes, as we verify by computing

$$
N_{\text {gauge }}=\frac{5}{2} \cdot \frac{1}{2}+\frac{1}{2} \cdot \frac{31}{4}+\frac{3}{2} \cdot \frac{13}{4}=10 .
$$

Next we parametrise the Kähler form $J=\sum_{i=1}^{5} K_{i}$ in terms of the five generators of the Kähler cone introduced previously, where compatibility with the orientifold action fixes $r_{1}=r_{2}$. One easily finds that the general solution to the D-term supersymmetry conditions relates the so-defined Kähler $r_{1}, r_{2}$ as well as the $B_{-}$-modulus $B_{-}=b\left(D_{7}-D_{8}\right)$ to $r_{4}, r_{5}$ as

$$
r_{1}=\frac{2}{3} r_{4}-r_{5}, \quad r_{3}=\frac{13}{6} r_{4}-2 r_{5}, \quad B_{-}=\frac{34 r_{4}-27 r_{5}}{38 r_{4}-30 r_{5}} .
$$

Remarkably any such choice of Kähler form lies inside the Kähler cone as long as $r_{5}>0$ and $\frac{3}{2} r_{5} \leq r_{4} \leq 2 r_{5}$. The existence of solutions inside the Kähler cone is, together with the appearance of exactly three generations of Standard Model matter, one of the motivations to present this example.

However, two caveats require further attention. As the reader is by now very familiar with, we break $S U(5)$ further to the Standard Model gauge group by turning on the line bundle $\mathcal{L}_{Y}$ on $D_{a}$ that is trivial on the ambient Calabi-Yau space. As before the $E_{6}$ sublattice within $H^{2}\left(D_{a}\right)$ of the $\mathrm{dP}_{9}$ surface $D_{a}$ is trivial on the Calabi-Yau, and the minimal choice for $\mathcal{L}_{Y}$ that avoids extra vector-like states is, for example, $\mathcal{L}_{Y}=\mathcal{O}\left(e_{1}-e_{2}\right)$. This, however, leads to an extra contribution of +2 in $N_{\text {gauge }}$ appearing in the D3-brane tadpole equation (228). In addition, the by 
now familiar twist of $L_{a}$ by $R_{a}=\mathcal{L}_{Y}^{-1}$ requires to engineer one Higgs doublet and to project out the triplet contributes with +1 on the left-hand side of equation (228). In conclusion, there is a total overshooting by three units in the D3-brane tadpole equation, which requires the introduction of three anti- $D_{3}$ branes.

A second subtlety is associated with cancellation of K-theory charge. Applying our probe argument we note that the only invariant candidate cycles for SP ChanPaton factors are $D_{1}, D_{4}, D_{5}, D_{6}$ and $D_{9}$, each of which is non-Spin. In view of the $B_{+}$-flux eq. (226) none of them can carry an invariant line bundle in agreement with the Freed-Witten quantisation condition except $D_{9}$. In fact, any line bundle of the form $L_{9}^{(n)}=\frac{n}{2}\left(D_{7}-D_{8}\right)$ with $n$ odd is a liable and invariant gauge field configuration. As stressed several times by now, in absence of unambiguous CFT techniques to establish the orientifold action in the invariant sector it is hard to decide if $\left(D_{9}, L_{9}^{(n)}\right)$ carries $S P$ or rather $S O$ Chan-Paton factors. In the first case, its worldvolume theory would suffer from a global Witten anomaly in our three-generation model, as can be verified by computing that the number of fundamental representations under the symplectic gauge group is odd. In this case, the probe argument would suggest that model would not be globally consistent due to the non-cancellation of K-theory charge. We do not attempt to settle this issue at this stage but rather leave this model in the limbo of phenomenologically highly appealing configurations whose liability as a genuine string vacuum hinges upon as subtle and innocent a condition as the cancellation of torsion K-theory charge. With this warning in mind we conclude our model building adventures with a summary of the phenomenological properties of this model in Table 19.

\section{Comments on Moduli Stabilisation}

So far we have focused our attention on the construction of realistic $S U(5)$ GUT models on intersecting D7-branes in Type IIB orientifold models. Eventually, to obtain a truly predictive framework we have to address the central question of moduli stabilisation. Luckily, just for this kind of models very powerful techniques for moduli stabilisation have been developed during the last years. First to mention is the possibility of freezing the complex structure moduli and the dilaton via three-form fluxes inducing a Gukov-Vafa-Witten type superpotential. Combining this with D3instanton induced contributions depending on the Kähler moduli very predictive scenarios with in principle all moduli stabilised have been proposed. These include in particular the original KKLT scenario [6] with supersymmetry breaking via an 


\begin{tabular}{|c||c|c|}
\hline \hline property & mechanism & status \\
\hline \hline globally consistent & tadpoles + K-theory & $\checkmark^{* * * *}$ \\
D-term susy & vanishing FI-terms inside Kähler cone & $\checkmark$ \\
gauge group $S U(5)$ & $U(5) \times U(1)$ stacks & $\checkmark$ \\
3 chiral generations & choice of line bundles & $\checkmark$ \\
no vector-like matter & localisation on $g=0,1$ curves & $\checkmark$ \\
5 vector-like Higgs & choice of line bundles & $\checkmark$ \\
no adjoints & rigid 4 -cycles, del Pezzo & $\checkmark$ \\
GUT breaking & $U(1)_{Y}$ flux on trivial 2-cycles & $\checkmark$ \\
$3-2$ splitting & Wilson lines on $g=1$ curve & $\checkmark$ \\
3 split + no dim=5 p-decay & local. of $H_{u}, H_{d}$ on disjoint comp. & - \\
$\mathbf{1 0} \overline{\mathbf{5}} \overline{\mathbf{5}}_{\mathbf{H}}$ Yukawa & perturbative & $\checkmark$ \\
$\mathbf{1 0} \mathbf{1 0} \mathbf{5}_{\mathbf{H}}$ Yukawa & presence of appropriate D3-instanton & $-{ }^{* * *}$ \\
Majorana neutrino masses & presence of appropriate D3-instanton & $-{ }^{* * *}$ \\
\hline \hline
\end{tabular}

Table 19: Summary of $S U(5)$ properties realised in the model of Subsection 7.3.

* overshooting in D3-tadpole $\rightarrow 3 \overline{D 3}$-branes

** K-theory to the best of our ability to detect SP-cycles and modulo the possible issue of $\left(D_{9}, L_{9}^{(n)}\right)$

*** at least not with $O(1)$ instantons 
uplift potentia26. In some respects even better controlled is the LARGE volume scenario with Kähler moduli dominated supersymmetry breaking [7,8].

In this latter scenario it is essential to have a Calabi-Yau with negative Euler characteristic (that is, $h^{2,1}>h^{1,1}$ ) and shrinkable, rigid four-cycles supporting D3brane instantons contributing to the superpotential. Such cycles are given by del Pezzo surfaces $\mathrm{dP}_{n}$ with $n \leq 8$. Therefore, gauge coupling unification with $S U(5)$ breaking via $U(1)_{Y}$ fluxes and controllable moduli stabilisation with natural supersymmetry breaking both lead us to the class of Type IIB orientifolds (with some four-cycles $T_{Y u k} \rightarrow 0$ ) on Calabi-Yau manifolds which contain $\mathrm{dP}_{n}$ surfaces supporting two-cycles which are trivial in the Calabi-Yau manifold.

We have already discussed in Subsection 4.5 that the $M_{n}^{\left(\mathrm{dP}_{8}\right)^{n}}$ manifolds exhibit the swiss-cheese structure of the volume form. Thus, the class of Calabi-Yau manifolds studied in Sections 4, 5, and 6 as promising candidates for GUT model building likewise exhibits some attractive features for LARGE volume moduli stabilisation. For the $M_{n}^{\left(\mathrm{dP}_{8}\right)^{n}}$ manifolds we have also shown that placing the $S U(5)$ GUT on a shrinkable $\mathrm{dP}_{8}$ cycle, the D-terms force this cycle to shrink to string scale size. If one is not deterred by the appearance of quantum corrections, one can consider this either as a global embedding of local quiver theorie 27 . If one tries to avoid such corrections the above observation can alternatively serve as a motivation to place the GUT branes on del Pezzo $\mathrm{dP}_{9}$ or other non-shrinkable rigid surfaces instead. In this latter case, the D-term constraints can be solved in the large radius regime. This results in a scenario where the GUT branes are localised on $\mathrm{dP}_{9}$ surfaces while instantons on $\mathrm{dP}_{8}$ or lower del Pezzo surfaces can generate the superpotential contributions realising the LARGE volume scenario. At this stage we also point out that for consistency in a GUT model the string scale must be fixed not below the GUT scale, of course. Thus in our context the original LARGE volume scenario, if applied, has to be modified anyway as to stabilise the volume of the manifold at not too LARGE values $\mathcal{V} \simeq 10^{4}$. For a scenario leading to $M_{s}=M_{G U T}$ along these lines see [10].

The arrangement just described also resolves the constraints pointed out in [61] for the coexistence of a chiral MSSM or GUT like intersecting D7-brane sector on the one hand and of a D3-brane instanton sector contributing to the uncharged superpotential on the other. Since here the phenomenologically relevant sizes of

\footnotetext{
${ }^{26}$ In this sense the presence of anti-D 3 branes in some of our models might turn out to be some use.

${ }^{27}$ While this work was in its final stages the authors of [7] proposed a very similar scenario.
} 
the D7-brane cycles are fixed by the D-term constraints instead of the F-terms, the resulting soft-terms and low-energy signatures are expected to be different from the ones computed so far in the literature for the LARGE volume scenario [8, 79, 9, 10]. There, it was mostly assumed that the string scale is in the intermediate regime and that the MSSM supporting D7-branes wrap the same cycle as the D3-brane instanton.

Very similar conclusions follow from the analysis of the quintic descendants. In contrast to the models derived from $\mathbb{P}_{1,1,1,6,9}[18]$, here we have found intersecting $\mathrm{dP}_{n}$, $n \leq 8$ surfaces, which therefore do not show a swiss cheese structure and allow the Dterms to freeze the Kähler moduli such that the volumes of these del Pezzo remain finite. To arrange for the LARGE or rather GUT volume scenario for this class of models additional points at generic positions have to be blown up, presumably resulting in additional del Pezzo divisors, which are orthogonal to ones supporting D7-branes.

The next logical step is to combine fluxes, instanton effects and GUT D7-brane sectors such that a completely realistic and predictive model arises. For this purpose, one first needs to study the coexistence of three-form fluxes and D7-branes on the same Calabi-Yau, for which additional consistency conditions arise. Here to mention is both the Freed-Witten condition $\left.H_{3}\right|_{D}=0$ and a possible change for the quantisation of the gauge fluxes due to the presence of $F_{3}$ form flux [80]. Moreover, also the coexistence of Euclidean D3-brane instanton contributions to the superpotential and the desired presence of a chiral GUT D7-brane sector implies additional constraints [61]. All this to be evaluated and taken into account carefully to claim to have realised the MSSM or a variation therefore, from a string compactification.

This is a formidable task, but not out of reach in the not too far future. We think the results reported in this paper on GUT realisations in Type IIB orientifolds provide an encouraging step towards achieving this goal.

\section{Conclusions}

In this paper we have started to systematically analyse the construction of GeorgiGlashow like $S U(5)$ GUTs from Type IIB orientifolds with D7- and D3-branes. First, we formulated the quite restrictive global model building rules. Beyond the common tadpole and K-theory constraints, there arise a couple of additional subtle but quite restrictive constraints. These include the delicate quantisation rules for the gauge 
flux on the D7-branes wrapping rigid del Pezzo surfaces, which derive from the fact that del Pezzo surfaces are not Spin. In addition these gauge fluxes have to be chosen such that the D-term constraints can be satisfied inside the Kähler cone of the Calabi-Yau threefold. Applied to $S U(5)$ GUT models, in particular the quantisation conditions cannot be satisfied with only the GUT breaking line bundle $\mathcal{L}_{Y}$ supported on the $S U(5)$ stack. The presence of a second bundle $\mathcal{L}_{a}$ embedded into the diagonal $U(1) \subset U(5)$ is essential. It would be interesting to study the precise lift of the consistency conditions to the F-theory description of these models. While some details are known, we think it is fair to say that the general picture is still not fully understood.

After outlining the general structure, we have provided a class of concrete CalabiYau threefolds containing del Pezzo surfaces. Though the construction is more general, we first considered examples descending from the Calabi-Yau manifold $\mathbb{P}_{1,1,1,6,9}[18]$ via del Pezzo transitions. The resulting Calabi-Yau threefolds feature various phases (triangulations) related via flop-transitions of curves in the del Pezzo base. To define orientifolds of Type IIB on these manifolds, we have classified all their involutions resulting from involutions of the del Pezzo base. This provides already a large set of models, which deserves a more systematic (statistical) investigation than we could provide in this paper. Clearly, there exist more general involutions which also act on the elliptic fibre. The prototype example is just the $y \rightarrow-y$ involution of the elliptic fibre. It would be interesting to study these more general orientifolds, as well. Another natural route to pursue is to start with the related torus fibred Calabi-Yau manifolds $\mathbb{P}_{1,1,1,3,6}[12]$ and $\mathbb{P}_{1,1,1,3,3}[9]$. More generally, one could study systematically which Calabi-Yau manifolds in the known class of hypersurfaces in toric varieties allow for similar del Pezzo transitions.

Equipped with the general structure and appropriate concrete Calabi-Yau manifolds, we have manually searched for globally consistent examples. We have presented three models in detail, each realising around $60-70 \%$ of the desired GUT features, with almost every property being realised in at least one example. Therefore, we do not see any conceptual obstacle to finding GUT models exhibiting all features in a single configuration.

We have also introduced a second class of suitable Calabi-Yau manifolds defined via del Pezzo transitions of the simple quintic hypersurface in $\mathbb{P}_{4}$. In particular, these manifolds contain intersecting shrinkable del Pezzo surfaces, a property the first class based on $\mathbb{P}_{1,1,1,6,9}[18]$ was lacking due to the swiss-cheese structure of the triple intersection form. Finally, we have presented two GUT models with all matter 
fields localised on curves and therefore without any additional vector-like matter, the second of which realises just three families of Standard Model matter plus a Higgs pair. Clearly, it would be interesting to generalise the here presented techniques for constructing (toric) Calabi-Yau manifolds containing del Pezzo surfaces.

Our emphasis has been on the global string consistency conditions, which in a first attempt seem to be easier to analyse in the IIB orientifold framework than for F-theory compactifications on compact elliptically fibred Calabi-Yau fourfolds. The price one has to pay for working in the orientifold phase is that some couplings such as top-Yukawa couplings and Majorana neutrino masses are non-perturbatively generated by D3-brane instantons. With the recent understanding of such instanton effects we have however been able to formulate a criterion respectively constraint for their presence in concrete set-ups. The realistic corner in the moduli space of these models is clearly where the 4-cycles wrapped by these instantons go to zero size. In this respect it would be very important to better understand the relation of the orientifold construction to the F-theory uplift on Calabi-Yau fourfolds.

Eventually, we have briefly discussed the issue of moduli stabilisation for these models. We have shown that the manifolds $M_{n}^{\left(\mathrm{dP}_{8}\right)^{n}}$ indeed feature a swiss-cheese structure, which is a prerequisite for realising the LARGE volume scenario. We think it is striking that both from the viewpoint of realising GUTs and from the viewpoint of phenomenologically acceptable moduli stabilisation one is led to the same class of string constructions, namely Type IIB orientifolds (F-theory) on CalabiYau threefolds with shrinkable four-cycles, that is, del Pezzo surfaces.

\section{Acknowledgements}

We gratefully acknowledge helpful discussions with Andres Collinucci, Mirjam Cvetič, Hans Jockers, Shamit Kachru, Albrecht Klemm, Dieter Lüst, Sebastian Moster, Hans Peter Nilles, Erik Plauschinn, Maximilian Schmidt-Sommerfeld and Gary Shiu. Furthermore we thank the CERN Theory Institute for hospitality during parts of this work. R.B., V.B. and T.G. acknowledge the hospitality of the Erwin-SchrödingerInstitut, Vienna, and T.W. thanks the Aspen Center for Theoretical Physics and the Max-Planck-Institut, Munich, for hospitality during parts of this project. This work was supported in parts by the European Union 6th framework program MRTNCT-2004-503069 "Quest for unification", MRTN-CT-2004-005104 "ForcesUniverse", MRTN-CT-2006-035863 "UniverseNet", SFB-Transregio 33 "The Dark Universe" by the DFG and by the US Department of Energy under contract DE-AC02-76SF00515. 


\section{Appendices}

\section{A Involutions on Del Pezzo Surfaces}

\section{A.1 Del Pezzo Surfaces of High Degree}

This appendix is the completion and continuation of Subsection 4.1. We start with a more detailed examination of the del Pezzo surfaces of high degree $\geq 6$. In the Subsections A.2 A.6 we will consider each such del Pezzo surface individually and classify their involution 28 . However, before we go into the details of the different involutions let us recall Table 20

\section{A.2 Involutions on the Projective Plane}

Let us start with the simplest del Pezzo surface, $\mathbb{P}^{2}$. There are no $(-1)$-curves. Up to coordinate transformations, the unique involution acts on the homogeneous coordinates as

$$
\sigma: \mathbb{P}^{2} \rightarrow \mathbb{P}^{2}, \quad\left[z_{0}: z_{1}: z_{2}\right] \mapsto\left[-z_{0}: z_{1}: z_{2}\right] .
$$

The fixed point set of the involution $\sigma$ is

$$
\left(\mathbb{P}^{2}\right)^{\sigma}=\{[0: *: *]\} \cup\{[1: 0: 0]\} \simeq \mathbb{P}^{1} \cup\{\mathrm{pt}\},
$$

and its homology class is $l \in H_{2}\left(\mathbb{P}^{2}, \mathbb{Z}\right)$.

The projective plane is a toric variety, determined by the 2-dimensional polytope shown in Figure 4 as follows. Associate one complex-valued variable to each point of the polytope. Here, we label them $x_{0}, x_{1}$, and $x_{2}$. Whenever there are two points that are not connected by a line, the two variables are not allowed to vanish simultaneously. This does not happen here, but will be important later on. Finally, for each linear relation amongst the points we impose the an equivalence under "homogeneous" rescaling. For example, the single linear relation

$$
\begin{aligned}
\overbrace{(0,1)}^{x_{0}}+\overbrace{(-1,-1)}^{x_{1}}+\overbrace{(1,0)}^{x_{2}} & =0 \\
& \Rightarrow \quad\left[x_{0}: x_{1}: x_{2}\right]=\left[\lambda x_{0}: \lambda x_{1}: \lambda x_{2}\right] \quad \forall \lambda \in \mathbb{C}^{\times}
\end{aligned}
$$

\footnotetext{
${ }^{28}$ That is, the different connected components of the moduli space of involutions.
} 


\begin{tabular}{|c|c|c|c|c|c|c|}
\hline$S$ & $\operatorname{deg}(S)$ & case & action & fixed point set $S^{\sigma}$ & {$\left[S^{\sigma}\right] \in H_{2}$} & action on $H_{2}(S, \mathbb{Z})$ \\
\hline $\mathbb{P}^{2}$ & 9 & 1 & eq. (231) & {$[0: *: *] \cup[1: 0: 0] \simeq \mathbb{P}^{1} \cup\{$ pt. $\}$} & $l$ & $(1)$ \\
\hline $\mathbb{P}^{1} \times \mathbb{P}^{1}$ & 8 & 1 & eq. (237a) & {$[1: 1 \mid *: *] \cup[-1: 1 \mid *: *]$} & $\left(l_{2}\right)+\left(l_{2}\right)$ & $\left(\begin{array}{ll}1 & 0 \\
0 & 1\end{array}\right)$ \\
\hline $\mathbb{P}^{1} \times \mathbb{P}^{1}$ & 8 & 2 & eq. (237b & {$[ \pm 1: 1 \mid \pm 1: 1] \simeq 4$ points } & 0 & $\left(\begin{array}{ll}1 & 0 \\
0 & 1\end{array}\right)$ \\
\hline $\mathbb{P}^{1} \times \mathbb{P}^{1}$ & 8 & 3 & eq. (237c) & diagonal $\mathbb{P}^{1}$ & $l_{1}+l_{2}$ & $\left(\begin{array}{ll}0 & 1 \\
1 & 0\end{array}\right)$ \\
\hline $\mathcal{B}_{1}$ & 8 & 1 & eq. (241a) & $\pi^{-1}([0: *: *]) \cup e_{1} \simeq 2 \mathbb{P}^{1}$ & $(l)+\left(e_{1}\right)$ & $\left(\begin{array}{ll}1 & 0 \\
0 & 1\end{array}\right)$ \\
\hline $\mathcal{B}_{1}$ & 8 & 2 & eq. (241b) & $\mathbb{P}^{1} \cup\{2$ pts. $\}$ & $l-e_{1}$ & $\left(\begin{array}{ll}1 & 0 \\
0 & 1\end{array}\right)$ \\
\hline $\mathcal{B}_{2}$ & 7 & 1 & eq. (248) & $2 \mathbb{P}^{1} \cup\{$ pt. $\}$ & $\left(l-e_{1}\right)+\left(e_{2}\right)$ & $\left(\begin{array}{lll}1 & 0 & 0 \\
0 & 1 & 0 \\
0 & 0 & 1\end{array}\right)$ \\
\hline $\mathcal{B}_{2}$ & 7 & 2 & eq. (251) & $\mathbb{P}^{1} \cup\{3$ pts. $\}$ & $l-e_{1}-e_{2}$ & 0 \\
\hline $\mathcal{B}_{2}$ & 7 & 3 & eq. (252) & $\mathbb{P}^{1} \cup\{$ pt. $\}$ & $l$ & $\left(\begin{array}{llll}1 & 0 \\
0 & 0 & 0 \\
0 & 1 & 1\end{array}\right]$ \\
\hline $\mathcal{B}_{3}$ & 6 & 1 & eq. (255) & $2 \mathbb{P}^{1} \cup\{2$ pts. $\}$ & $\left(e_{1}\right)+\left(l-e_{2}-e_{3}\right)$ & \\
\hline $\mathcal{B}_{3}$ & 6 & 2 & eq. (258) & $\mathbb{P}^{1} \cup\{2$ pts. $\}$ & $l-e_{3}$ & $\begin{array}{llll}1 & 0 & 0 & 0 \\
0 & 0 & 1 & 0 \\
0 & 1 & 0 & 0 \\
0 & 0 & 0\end{array}$ \\
\hline $\mathcal{B}_{3}$ & 6 & 3 & eq. (259) & $\mathbb{P}^{1}$ & $2 l-e_{1}-e_{2}$ & $\left(\begin{array}{cccc}2 & 1 & 1 & 1 \\
-1 & -1 & 0 & -1 \\
-1 & 0 & -1 & -1 \\
-1 & -1 & -1 & 0\end{array}\right)$ \\
\hline $\mathcal{B}_{3}$ & 6 & 4 & eq. (261) & 4 points & 0 & $\left(\begin{array}{cccc}2 & 1 & 1 & 1 \\
-1 & 0 & -1 & -1 \\
-1 & -1 & 0 & -1 \\
-1 & -1 & -1 & 0\end{array}\right)$ \\
\hline
\end{tabular}

Table 20: Involutions on del Pezzo surfaces of degree $\geq 6$. 
corresponds to the usual rescaling of the homogeneous coordinates. Hence, the toric description of the projective plane is

$$
\frac{\mathbb{C}^{3}-\{\}}{\mathbb{C}^{\times}}=\mathbb{P}^{2} .
$$

By construction, the "algebraic torus" $\left(\mathbb{C}^{\times}\right)^{3}$ act 29 multiplicatively on the 3 homogeneous variables of $\mathbb{P}^{2}$, hence the name toric variety. Any subgroup of this action is called a toric group action. In particular, the involution eq. (231) is a toric $\mathbb{Z}_{2}$ action.

Alternatively, the involution can be seen as a symmetry of the polytope. The reflection symmetry shown in Figure 4 generates the involution

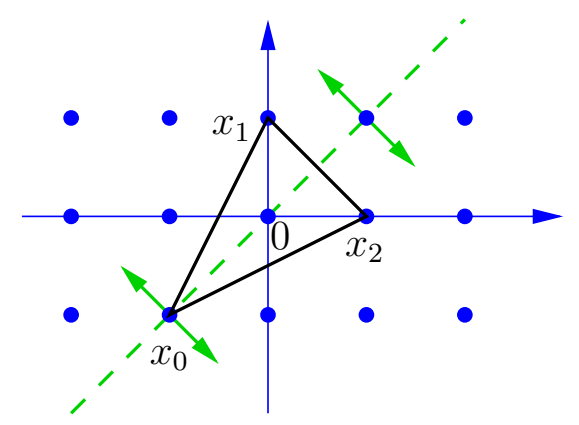

Figure 4: Symmetry of the toric polytope defining $\mathbb{P}^{2}$.

$$
\sigma: \frac{\mathbb{C}^{3}}{\mathbb{C}^{\times}} \rightarrow \frac{\mathbb{C}^{3}}{\mathbb{C}^{\times}}, \quad\left[x_{0}: x_{1}: x_{2}\right] \mapsto\left[x_{0}: x_{2}: x_{1}\right]
$$

This is the same group action as in eq. (231), only written in different coordinates 30 .

\section{A.3 Involutions on the Product of Lines}

There is only one non-trivial involution on $\mathbb{P}^{1}$ acting as $\left[z_{0}: z_{1}\right] \mapsto\left[-z_{0}: z_{1}\right]$, which can act on each factor of $\mathbb{P}^{1} \times \mathbb{P}^{1}$. Together with the exchange of the two factors, this

\footnotetext{
${ }^{29}$ Clearly, the diagonal $\mathbb{C}^{*}$ is already modded out and acts trivially.

${ }^{30}$ For future reference, we note that the fixed point set in the coordinates eq. (235) is

$$
\left(\mathbb{P}^{2}\right)^{\sigma}=\left\{\left[t_{0}: t_{1}: t_{1}\right] \mid\left[t_{0}: t_{1}\right] \in \mathbb{P}^{1}\right\} \cup\{[0: 1:-1]\} \simeq \mathbb{P}^{1} \cup\{\mathrm{pt}\} .
$$
}


generates all possible holomorphic involutions on $\mathbb{P}^{1} \times \mathbb{P}^{1}$. All of these involutions arise from symmetries of the toric polytope, see Figure 5. The symmetry group of

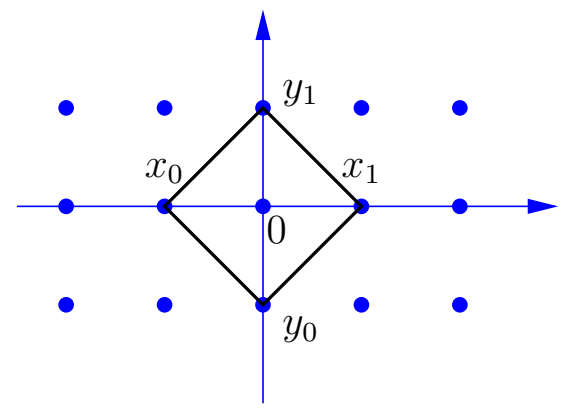

Figure 5: The toric polytope defining $\mathbb{P}^{1} \times \mathbb{P}^{1}$.

the toric polytope is $D_{8}$, the dihedral group with 8 elements. It has three conjugacy classes of $\mathbb{Z}_{2}$ subgroups, namely:

1. Mirroring at vertical axis. In homogeneous coordinates, the induced action on $\mathbb{P}^{1} \times \mathbb{P}^{1}$ is

$$
\sigma_{1}: \mathbb{P}^{1} \times \mathbb{P}^{1} \rightarrow \mathbb{P}^{1} \times \mathbb{P}^{1}, \quad\left[x_{0}: x_{1} \mid y_{0}: y_{1}\right] \mapsto\left[x_{1}: x_{0} \mid y_{0}: y_{1}\right] .
$$

2. Rotating by $\pi$, with induced action

$$
\sigma_{2}: \mathbb{P}^{1} \times \mathbb{P}^{1} \rightarrow \mathbb{P}^{1} \times \mathbb{P}^{1}, \quad\left[x_{0}: x_{1} \mid y_{0}: y_{1}\right] \mapsto\left[x_{1}: x_{0} \mid y_{1}: y_{0}\right] .
$$

3. Mirroring at diagonal axis $=$ Rotate by $\frac{\pi}{2}$ and mirror at vertical axis. The induced action is

$$
\sigma_{3}: \mathbb{P}^{1} \times \mathbb{P}^{1} \rightarrow \mathbb{P}^{1} \times \mathbb{P}^{1}, \quad\left[x_{0}: x_{1} \mid y_{0}: y_{1}\right] \mapsto\left[y_{0}: y_{1} \mid x_{0}: x_{1}\right] .
$$

According to the Künneth theorem, the homology group $H_{2}\left(\mathbb{P}^{1} \times \mathbb{P}^{1}\right)=\mathbb{Z}^{2}$ is generated by the classes of the two factors, which we call $l_{1}$ and $l_{2}$. The fixed point sets and their homology classes are straightforward and listed in Table 20.

\section{A.4 Blow-up of the Projective Plane}

We now come to the first case with a $(-1)$-curve, namely the blow-up $\mathcal{B}_{1}$ of $\mathbb{P}^{2}$ at one point. One possible realisation is the hypersurface

$$
\mathcal{B}_{1}=\left\{x_{0} \cdot 0+x_{1} t_{0}+x_{2} t_{1}=0\right\} \subset \mathbb{P}_{\left[x_{0}: x_{1}: x_{2}\right]}^{2} \times \mathbb{P}_{\left[t_{0}: t_{1}\right]}^{1} .
$$


The obvious projection $\pi: \mathcal{B}_{1} \rightarrow \mathbb{P}^{2},\left[x_{0}: x_{1}: x_{2} \mid t_{0}: t_{1}\right] \mapsto\left[x_{0}: x_{1}: x_{2}\right]$ is, in fact, the corresponding blow-down map. To see this, consider the preimage:

- If $\left[x_{0}: x_{1}: x_{2}\right] \neq[1: 0: 0]$, then the preimage is the single point

$$
\pi^{-1}\left(\left[x_{0}: x_{1}: x_{2}\right]\right)=\left[x_{0}: x_{1}: x_{2} \mid x_{2}:-x_{1}\right]
$$

- If $\left[x_{0}: x_{1}: x_{2}\right]=[1: 0: 0]$, then the preimage is

$$
\pi^{-1}([1: 0: 0])=\left\{\left[1: 0: 0 \mid t_{0}: t_{1}\right] \mid\left[t_{0}: t_{1}\right] \in \mathbb{P}^{1}\right\} \simeq \mathbb{P}^{1}
$$

In other words, the hypersurface eq. (238) is the blow-up at $[1: 0: 0] \in \mathbb{P}^{2}$.

We now consider involutions of the hypersurface induced from the ambient space $\mathbb{P}^{2} \times \mathbb{P}^{1}$. In fact, up to coordinate changes there are two distinct possibilities, namely

$$
\sigma_{1}: \mathcal{B}_{1} \rightarrow \mathcal{B}_{1}, \quad\left[x_{0}: x_{1}: x_{2} \mid t_{0}: t_{1}\right] \mapsto\left[-x_{0}: x_{1}: x_{2} \mid t_{0}: t_{1}\right]
$$

and

$$
\sigma_{2}: \mathcal{B}_{1} \rightarrow \mathcal{B}_{1}, \quad\left[x_{0}: x_{1}: x_{2} \mid t_{0}: t_{1}\right] \mapsto\left[x_{0}: x_{2}: x_{1} \mid t_{1}: t_{0}\right] .
$$

In terms of the blown-up $\mathbb{P}^{2}$, the two involutions can be understood as follows. Recall from Subsection A.2 that the fixed-point set on $\mathbb{P}^{2}$ is the disjoint union of a line and a point.

1. The first involution, eq. (241a), is the blow-up of the isolated fixed point on $\mathbb{P}^{2}$. The corresponding fixed-point set in $\mathcal{B}_{1}$ is the whole exceptional $\mathbb{P}^{1}$ as well as the fixed line in $\mathbb{P}^{1}$. This $\mathbb{Z}_{2}$ group action is toric.

2. The second involution, eq. (236), is the blow-up at a point on the fixed line on $\mathbb{P}^{2}$. The exceptional $\mathbb{P}^{1}$ is mapped to itself, but it is not point-wise fixed. Rather, the involution acts as a rotation by $\pi$ on this $\mathbb{P}^{1} \simeq S^{2}$ and the north and south pole of the sphere end up being fixed. Looking at the whole $\mathcal{B}_{1}$, the proper transform of the fixed line in $\mathbb{P}^{2}$ passes through one of the fixed points in the exceptional curve. Hence, the fixed point set consists of this proper transform $31 \tilde{l} \simeq \mathbb{P}^{1}$ together with the remaining fixed point in the exceptional curve and the isolated fixed point that was already in $\mathbb{P}^{2}$.

In terms of toric geometry, this involution is induced from the reflection symmetry of the polyhedron shown in Figure 6

\footnotetext{
${ }^{31}$ The $\sigma_{2}$-fixed line $l_{2}$ in $\mathbb{P}^{2}$ has the parametrisation$$
\xi \mapsto l_{2}(\xi)=[1: \xi: \xi], \quad \xi \in \mathbb{C} \cup\{\infty\} \simeq \mathbb{P}^{1} .
$$ 


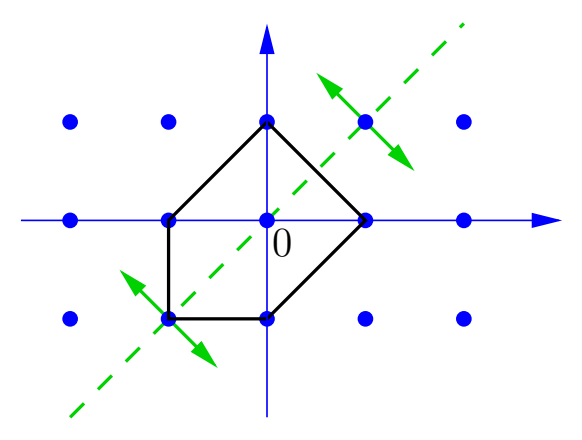

Figure 6: Symmetry of the toric polytope defining $\mathcal{B}_{1}$.

More abstractly, we can understand the two involutions from the $(-1)$-curves. Since there is precisely one such curve, namely $e_{1}$, this curve must necessarily be mapped to itself under any involution. But whenever there is an invariant 32 exceptional curve on $\mathcal{B}_{n}$, then we can blow down this curve and obtain a involution on $\mathcal{B}_{n-1}$ (or $\mathbb{P}^{1} \times \mathbb{P}^{1}$ if $n=2$ ). This is why every involution on $\mathcal{B}_{1}$ is simply the (unique) involution on $\mathbb{P}^{2}$ blown up at a fixed point. There are two connected components to the fixed point set, and the choice of blow-up point coincides with the two different ways that $e_{1} \simeq \mathbb{P}^{1}$ can be mapped to itself:

1. If one blows up the isolated fixed point on $\mathbb{P}^{2}$, then $e_{1}$ is point-wise fixed under the induced involution on $\mathcal{B}_{1}$.

2. If one blows up one point in the fixed line on $\mathbb{P}^{2}$, then the induced involution on $\mathcal{B}_{1}$ acts on $e_{1} \simeq S^{2}$ as rotation by $\pi$.

\section{A.5 Blow-up of the Projective Plane at Two Points}

The blow-up of $\mathbb{P}^{2}$ at two points, $\mathcal{B}_{2}$, is the first case with an interesting pattern of $(-1)$-curves. Clearly, there are the exceptional divisors $e_{1}$ and $e_{2}$. But there is also

It passes through the point $l_{2}(0)=[1: 0: 0]$, which we are about to blow up. By definition, the proper transform is the curve

$$
\tilde{l}=\pi^{-1} \circ l_{2}\left(\mathbb{P}^{1}-\{0\}\right) \cup \lim _{\xi \rightarrow 0}\left(\pi^{-1} \circ l_{2}(\xi)\right) \subset \mathcal{B}_{1} .
$$

Since $\tilde{l} \cdot l=1=\tilde{l} \cdot e_{1}$, the homology class of the proper transform must be $[\tilde{l}]=l-e_{1}$.

${ }^{32}$ One can of course blow down any $(-1)$-curve and obtain a smooth surface, but the involution is lost (or, rather, becomes a birational map) if the (-1)-curve was not invariant. 
a third rigid curve, namely the line on $\mathbb{P}^{2}$ through the two blow-up points. As we reviewed in Footnote 31, this line defines a curve on the blow-up $\mathcal{B}_{2}$. This so-called proper transform has homology class

$$
\tilde{l}=l-e_{1}-e_{2}
$$

One can easily check that $\tilde{l}^{2}=-1$, as expected for a rigid curve. We draw the intersection pattern of the three lines in Figure 7. In the following, we will always

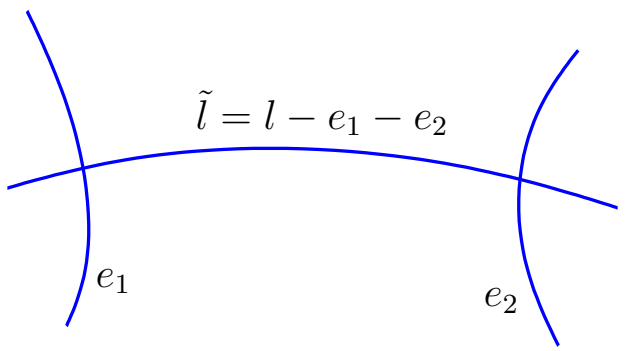

Figure 7: On the left, intersection pattern of the three (-1)-curves on $\mathcal{B}_{2}$. The dual graph is shown on the right.

use the dual graph of the (-1)-curves (and, by abuse of notation, drop the "dual"). By definition, this is the graph with

- One node for each (-1)-curve, and

- One connecting line whenever two curves intersect.

There are two different kinds of nodes, one of valence 2 and two of valence 1 . Blowing down the middle node yields $\mathbb{P}^{1} \times \mathbb{P}^{1}$, while blowing down one of the nodes at the end yields $\mathcal{B}_{1}$.

Clearly, the automorphism group of the graph is $\mathbb{Z}_{2}$ and the middle node is always fixed. Hence, the easiest way to describe all involutions is as blow-up of $\mathbb{P}^{1} \times \mathbb{P}^{1}$, where there were three distinct involutions. Just an in eq. (238), we will realise the blow-up at the point $\left[\xi_{0}: \xi_{1} \mid \eta_{0}: \eta_{1}\right] \in \mathbb{P}^{1} \times \mathbb{P}^{1}$ as a degree- $(1,1,1)$ hypersurface

$$
\mathcal{B}_{2}=\left\{\left(\xi_{1} x_{0}-\xi_{0} x_{1}\right) t_{0}+\left(\eta_{1} y_{0}-\eta_{0} y_{1}\right) t_{1}=0\right\} \subset \mathbb{P}_{\left[x_{0}: x_{1}\right]}^{1} \times \mathbb{P}_{\left[y_{0}: y_{1}\right]}^{1} \times \mathbb{P}_{\left[t_{0}: t_{1}\right]}^{1} .
$$

Using this construction, we can characterise the three different involutions on $\mathcal{B}_{2}$ as follows: 
1. First, let us start by blowing up $\left(\mathbb{P}^{1} \times \mathbb{P}^{1}, \sigma_{1}\right)$ at a $\sigma_{1}$-fixed point. The fixed point set consists of two disjoint $\mathbb{P}^{1}$, so one might think that there is a discrete choice. However, the two $\mathbb{P}^{1}$ are exchanged by a remaining symmetry of $\mathbb{P}^{1} \times \mathbb{P}^{1}$, so they cannot be distinguished. Henceforth, we will pick the fixed point

$$
p=[1: 1 \mid 0: 1] \in \mathbb{P}^{1} \times \mathbb{P}^{1}
$$

and define

$$
\mathcal{B}_{2}=\left\{\left(x_{0}-x_{1}\right) t_{0}+y_{0} t_{1}=0\right\} \subset \mathbb{P}_{\left[x_{0}: x_{1}\right]}^{1} \times \mathbb{P}_{\left[y_{0}: y_{1}\right]}^{1} \times \mathbb{P}_{\left[t_{0}: t_{1}\right]}^{1} .
$$

In order to make the hypersurface equation invariant under the involution, we must extend eq. (237a) to

$$
\sigma_{1}: \mathcal{B}_{2} \rightarrow \mathcal{B}_{2}, \quad\left[x_{0}: x_{1}\left|y_{0}: y_{1}\right| t_{0}: t_{1}\right] \mapsto\left[x_{1}: x_{0}\left|y_{0}: y_{1}\right|-t_{0}: t_{1}\right]
$$

2. Now we blow up one of the 4 fixed points of $\left(\mathbb{P}^{1} \times \mathbb{P}^{1}, \sigma_{2}\right)$. Again, the fixed points are exchanged by residual symmetries, and cannot be distinguished. Hence, there is essentially only one choice which we take to be

$$
p=[1: 1 \mid 1: 1] \in \mathbb{P}^{1} \times \mathbb{P}^{1} .
$$

The blow-up with the induced involution is then

$$
\begin{gathered}
\mathcal{B}_{2}=\left\{\left(x_{0}-x_{1}\right) t_{0}+\left(y_{0}-y_{1}\right) t_{1}=0\right\} \subset \mathbb{P}_{\left[x_{0}: x_{1}\right]}^{1} \times \mathbb{P}_{\left[y_{0}: y_{1}\right]}^{1} \times \mathbb{P}_{\left[t_{0}: t_{1}\right]}^{1}, \\
\sigma_{2}: \mathcal{B}_{2} \rightarrow \mathcal{B}_{2}, \quad\left[x_{0}: x_{1}\left|y_{0}: y_{1}\right| t_{0}: t_{1}\right] \mapsto\left[x_{1}: x_{0}\left|y_{1}: y_{0}\right| t_{0}: t_{1}\right]
\end{gathered}
$$

3. Finally, we can blow-up one point on the fixed (diagonal) $\mathbb{P}^{1}$ in $\left(\mathbb{P}^{1} \times \mathbb{P}^{1}, \sigma_{3}\right)$. For concreteness, let us take the point in eq. (249), which is also fixed under $\sigma_{3}$. Hence, the hypersurface equation is the same as in eq. (250). However, the induced involution has to extend a different involution on $\mathbb{P}^{1} \times \mathbb{P}^{1}$, and must be

$$
\sigma_{3}: \mathcal{B}_{2} \rightarrow \mathcal{B}_{2}, \quad\left[x_{0}: x_{1}\left|y_{0}: y_{1}\right| t_{0}: t_{1}\right] \mapsto\left[y_{0}: y_{1}\left|x_{0}: x_{1}\right| t_{1}: t_{0}\right]
$$

Equivalently, the three involutions can be described as blow-ups of $\mathbb{P}^{2}$ at two points. Let us quickly go over this equivalent point of view. 
1. The first two involutions correspond to the trivial automorphism of the graph of $(-1)$-curves. Hence, one must blow up two fixed points of $\left(\mathbb{P}^{2}, \sigma\right)$. The first possibility is to blow up the isolated fixed point and one point on the fixed line in $\mathbb{P}^{2}$. As we saw in Subsection A.4, blowing up a point on the fixed $\mathbb{P}^{1}$ leaves the proper transform $\tilde{l}$ (in the homology class $l-e_{1}$ ) and one isolated point fixed. Blowing up the fixed point leads to a point-wise fixed exceptional divisor $e_{2}$.

2. The second involution is the blow-up of two points on the fixed $\mathbb{P}^{1}$ of $\left(\mathbb{P}^{2}, \sigma\right)$. The fixed point set consists of

- The proper transform of the fixed line. Its homology class is $l-e_{1}-e_{2}$.

- One isolated fixed point on $e_{1}$ and one on $e_{2}$.

- The isolated fixed point that was already on $\mathbb{P}^{2}$.

3. The last involution corresponds to the non-trivial automorphism of the graph. There, the involution must exchange the two blow-up points. The fixed point set is the same as on $\left(\mathbb{P}^{2}, \sigma\right)$.

Clearly, the first two involutions act trivially on $H_{2}\left(\mathcal{B}_{2}, \mathbb{Z}\right)$. The third involution exchanges $e_{1} \leftrightarrow e_{2}$ while leaving $l$ invariant.

\section{A.6 Blow-up of the Projective Plane at Three Points}

The del Pezzo surface $\mathcal{B}_{3}$ has 6 rigid lines, the 3 exceptional divisors together with the 3 lines connecting any pair of blow-up points. Their homology classes are

$$
e_{1}, e_{2}, e_{3}, l-e_{2}-e_{3}, l-e_{1}-e_{3}, l-e_{2}-e_{3} .
$$

The graph of $(-1)$-curves is a hexagon, whose automorphism group is $D_{12}$, the dihedral group with 12 elements. It has 3 conjugacy classes of order 2 , which are depicted in Figure 8. We now investigate which involutions on $\mathcal{B}_{3}$ give rise to each graph automorphism.

1. Let us start with the trivial action on the graph. This is necessarily the blowup of $\left(\mathbb{P}^{2}, \sigma\right)$ at three fixed points. Note, however, that by construction no three point can lie on any one line and, in particular, not on the fixed line. 


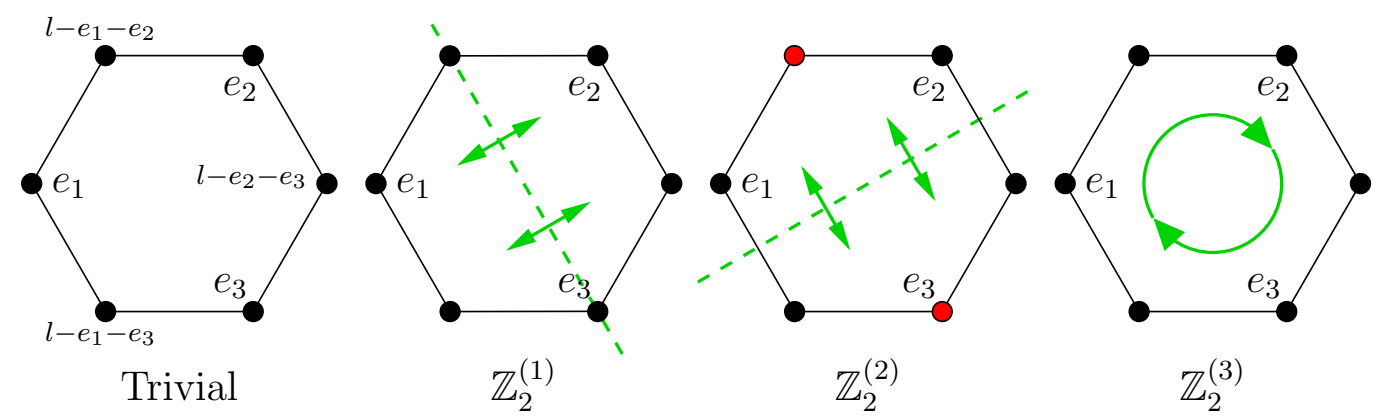

Figure 8: The trivial and the three order-2 automorphisms of the graph of $(-1)$-curves on $\mathcal{B}_{3}$.

Therefore, there is (up to coordinate changes) only one choice 33 of three fixed points to blow up. In the coordinates given by eq. (231), these three points can be chosen to be

$$
p_{1}=[1: 0: 0], p_{2}=[0: 1: 0], p_{3}=[0: 0: 1]
$$

The first involution on $\mathcal{B}_{3}$ is the one defined through the blow-up,

$$
\left(\mathcal{B}_{3}, \sigma_{1}\right)=\mathrm{Bl}_{\left\{p_{1}, p_{2}, p_{3}\right\}}\left(\mathbb{P}^{2}, \sigma\right) .
$$

We denote by $e_{i}$ the exceptional divisor of the blow-up at $p_{i}$. With this notation, the fixed point set consists of the exceptional divisor $e_{1}$, the proper transform of the fixed $\mathbb{P}^{1}$, one isolated point on $e_{2}$, and one isolated point on $e_{3}$.

2. We now consider the first non-trivial involution on the graph of $(-1)$-lines, which is denoted $\mathbb{Z}_{2}^{(1)}$ in Figure 8 , There are two fixed $(-1)$-curves, one of which we already labelled $e_{3}$. Blowing down this exceptional divisor $e_{3}$, we clearly obtain the surface $\mathcal{B}_{2}$ with the non-trivial action on its graph of $(-1)$ curves. There is only one such involution, namely $\left(\mathcal{B}_{2}, \sigma_{3}\right)$. Therefore, the desired involution on $\mathcal{B}_{3}$ is the blow-up of $\left(\mathcal{B}_{2}, \sigma_{3}\right)$ at a fixed point.

Recall that $\left(\mathcal{B}_{2}, \sigma_{3}\right)$ is the blow-up of $\left(\mathbb{P}^{2}, \sigma\right)$ at a point-image point pair. We can pick coordinates such that

$$
\left(\mathcal{B}_{2}, \sigma_{3}\right)=\mathrm{Bl}_{\{[1: 1: 0],[-1: 1: 0]\}}\left(\mathbb{P}^{2}, \sigma\right) .
$$

\footnotetext{
${ }^{33}$ Namely the isolated fixed point and two points on the fixed line in $\mathbb{P}^{2}$.
} 
The fixed point set of $\left(\mathcal{B}_{2}, \sigma_{3}\right)$ has two connected components, an isolated point $[1: 0: 0]$ and $[0: *: *] \simeq \mathbb{P}^{1}$. Note, however, that the isolated point is collinear with the previous blow-up points,

$$
\{[1: 1: 0],[-1: 1: 0],[1: 0: 0]\} \in\{[*: *: 0]\} .
$$

Hence, we cannot blow up $\left(\mathcal{B}_{2}, \sigma_{3}\right)$ at the isolated fixed point. The only possibility is to pick a fixed point $q \neq[1: 0: 0]$. This point $q$ must lie on the fixed $\mathbb{P}^{1}$ of $\left(\mathcal{B}_{2}, \sigma_{3}\right)$. Hence we obtain the involution

$$
\left(\mathcal{B}_{3}, \sigma_{2}\right)=\mathrm{Bl}_{q}\left(\mathcal{B}_{2}, \sigma_{3}\right)
$$

3. Now, consider the $\mathbb{Z}_{2}^{(2)}$-automorphism shown in Figure 8, There is no fixed $(-1)$-curve, so we cannot describe it in terms of a blown-up $\mathcal{B}_{2}$ del Pezzo. We can, however, blow down a pair of $(-1)$-curves that is exchanged by the involution and does not intersect. There is only one such pair, marked in red. Blowing down this pair yields a del Pezzo surface of degree 8 without any remaining (-1)-curves, that is, $\mathbb{P}^{1} \times \mathbb{P}^{1}$.

There are three choices for the involution on $\mathbb{P}^{1} \times \mathbb{P}^{1}$. It turns out 34 that $\left(\mathbb{P}^{1} \times \mathbb{P}^{1}, \sigma_{3}\right)$ gives rise to the right graph automorphism. Therefore, we set

$$
\left(\mathcal{B}_{3}, \sigma_{3}\right)=\mathrm{Bl}_{\{p, q\}}\left(\mathbb{P}^{1} \times \mathbb{P}^{1}, \sigma_{3}\right),
$$

where $p, q$ is a generic point and its image on $\left(\mathbb{P}^{1} \times \mathbb{P}^{1}, \sigma_{3}\right)$. The fixed point set $\mathcal{B}_{3}^{\sigma_{4}}$ is, by construction, the same as $\left(\mathbb{P}^{1} \times \mathbb{P}^{1}\right)^{\sigma_{3}}$. Since the $(-1)$-curves span $H_{2}\left(\mathcal{B}_{3}, \mathbb{Z}\right)$, the action on the curve homology classes can be read off from Figure 8. One obtains

$$
\left(\begin{array}{c}
l \\
e_{1} \\
e_{2} \\
e_{3}
\end{array}\right) \mapsto\left(\begin{array}{c}
2 l-e_{1}-e_{2}-e_{3} \\
l-e_{1}-e_{3} \\
l-e_{2}-e_{3} \\
l-e 1-e_{2}
\end{array}\right)
$$

4. Finally, consider $\mathbb{Z}_{2}^{(3)}$. Again, there is no $(-1)$-curve, but we can blow down a pair of $(-1)$-curves and relate the involution to $\mathbb{P}^{1} \times \mathbb{P}^{1}$. In fact, only one

\footnotetext{
${ }^{34} \mathrm{On}\left(\mathbb{P}^{1} \times \mathbb{P}^{1}, \sigma_{1}\right)$ there is no suitable pair of non-fixed points to blow up into $\mathcal{B}_{3}$. The second involution, $\left(\mathbb{P}^{1} \times \mathbb{P}^{1}, \sigma_{3}\right)$, will be used in Item 4.
} 
involution on $\mathbb{P}^{1} \times \mathbb{P}^{1}$ gives rise to the desired graph automorphism. Hence, we set

$$
\left(\mathcal{B}_{3}, \sigma_{4}\right)=\mathrm{Bl}_{\{p, q\}}\left(\mathbb{P}^{1} \times \mathbb{P}^{1}, \sigma_{2}\right),
$$

where $p, q$ is a generic point and its image on $\left(\mathbb{P}^{1} \times \mathbb{P}^{1}, \sigma_{2}\right)$.

\section{A.7 The Weyl Group and The Graph of Lines}

Recall that, by definition, the degree of a curve is its intersection with the canonical class $K=-3 l+\sum_{i} e_{i}$. As an alternative basis for the curve homology classes one
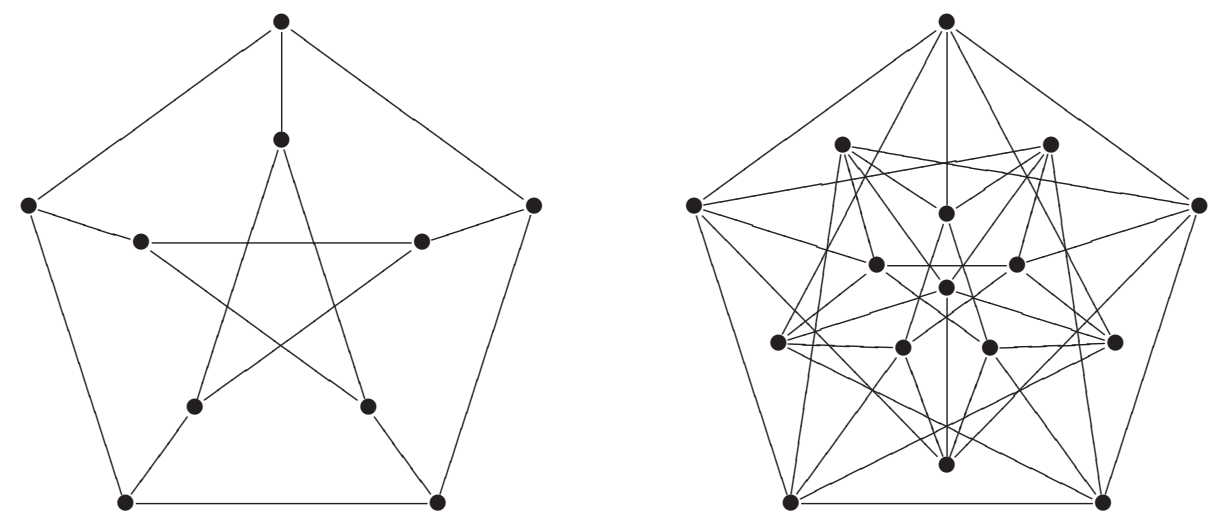

Figure 9: The Petersen graph (left) and the Clebsch graph (right).

can use the canonical class and the degree zero sublattice

$$
K^{\perp} \subset H_{2}\left(\mathcal{B}_{n}, \mathbb{Z}\right)
$$

This sublattice contains a finite number of classes with self-intersection -2 . One can show [81] that these classes span the root lattic 35 of a Lie algebra for $n \geq 2$. By this identification, we will call the (-2)-classes 36 of degree 0 roots, as well. Explicitly, the simple roots can be taken to be

$$
\begin{aligned}
\alpha_{i} & =e_{i}-e_{i+1}, \quad i=1, \ldots, n-1, \\
\alpha_{n} & =l-e_{1}-e_{2}-e_{3} .
\end{aligned}
$$

\footnotetext{
${ }^{35}$ The lattice product on $K^{\perp}$ is minus the intersection product in homology.

${ }^{36}$ Note that such a $(-2)$-homology class cannot be represented by a holomorphic curve on a del Pezzo surface.
} 


\begin{tabular}{|c|c|c|c|c|c|c|c|}
\hline del Pezzo $S$ & $\operatorname{deg}(S)$ & $\begin{array}{l}\# \text { of } \\
\text { roots }\end{array}$ & root lattice & Weyl group $W$ & Order $|W|$ & $\begin{array}{c}\text { Number of } \mathbb{Z}_{2} \\
\text { conjugacy classes }\end{array}$ & graph \\
\hline $\mathbb{P}^{2}$ & 9 & 0 & \{\} & 1 & 1 & 0 & \{\} \\
\hline $\mathbb{P}^{1} \times \mathbb{P}^{1}$ & 8 & 0 & \{\} & 1 & 1 & 0 & \{\} \\
\hline $\mathcal{B}_{1}$ & 8 & 0 & \{\} & 1 & 1 & 0 & $\bullet$ \\
\hline $\mathcal{B}_{2}$ & 7 & 2 & $A_{1}$ & $\mathbb{Z}_{2}$ & 2 & 1 & $\bullet \bullet \bullet$ \\
\hline $\mathcal{B}_{3}$ & 6 & 8 & $A_{2} \oplus A_{1}$ & $D_{6} \times \mathbb{Z}_{2}=D_{12}$ & 12 & 3 & \\
\hline $\mathcal{B}_{4}$ & 5 & 20 & $A_{4}$ & $S_{5}$ & 120 & 2 & $\begin{array}{c}\text { Petersen graph } \\
\text { Figure 9 }\end{array}$ \\
\hline $\mathcal{B}_{5}$ & 5 & 40 & $D_{5}$ & $\operatorname{Weyl}\left(D_{5}\right)$ & 1920 & 5 & $\begin{array}{c}\text { Clebsch graph } \\
\text { Figure 9 }\end{array}$ \\
\hline $\mathcal{B}_{6}$ & 5 & 72 & $E_{6}$ & $\operatorname{Weyl}\left(E_{6}\right)$ & 51840 & 4 & 27 nodes \\
\hline $\mathcal{B}_{7}$ & 5 & 126 & $E_{7}$ & $\operatorname{Weyl}\left(E_{7}\right)$ & 2903040 & 9 & 56 nodes \\
\hline $\mathcal{B}_{8}$ & 5 & 240 & $E_{8}$ & $\operatorname{Weyl}\left(E_{8}\right)$ & 696729600 & 9 & 240 nodes \\
\hline
\end{tabular}

Table 21: The Weyl groups and the number of $\mathbb{Z}_{2}$ conjugacy classes. The Weyl group equals the automorphism group of the graph of (-1)-curves on each del Pezzo surfaces. 
The intersection matrix is given in terms of the Cartan matrix $C_{i j}$ of the corresponding Lie algebra as

$$
\alpha_{i} \cdot \alpha_{j}=-C_{i j}, \quad \alpha_{i} \cdot K=0, \quad K^{2}=9-n .
$$

The root lattices for the del Pezzo surfaces are listed in Table 21 together with some information on the corresponding Weyl groups.

One of the advantages of working with this root lattice is the following characterisation of the symmetries of the graph of $(-1)$-curves on a del Pezzo surface:

Theorem 1 (Manin) The Weyl group of the root lattice associated to a del Pezzo surface equals the automorphism group of the graph of (-1)-curves.

In particular, we are interested in the conjugacy classes of involutions acting on the graph of $(-1)$-curves, which we can easily compute in terms of the conjugacy classes of $\mathbb{Z}_{2}$ subgroups in the corresponding Weyl group. It is now a simple (but tedious) computation to enumerate 37 all the Weyl group elements in each conjugacy class.

Note that, on del Pezzo surfaces of degree 6 and higher $\left(\mathcal{B}_{n}\right.$ with $\left.n \geq 3\right)$, the canonical class and root lattice span the whole homology groun $38 H_{2}\left(\mathcal{B}_{n}, \mathbb{Q}\right)$. From now on we will restrict ourselves to this case, where we now have three equivalent bases for the (rational) homology:

- The standard basis $l, e_{1}, \ldots, e_{n}$.

- The canonical class together with the $n$ simple roots.

- Any maximal (that is, consisting of $n+1$ ) linearly independent set of $(-1)$ curves.

The second basis is especially useful to determine the homology action of an involution on a del Pezzo surface. By definition, the canonical class is invariant under the action of a holomorphic map and the Weyl group acts on its orthogonal complement $K^{\perp}$. In Table 22, we use this to find the action on the homology of each possible involution of the graph of $(-1)$-curves. Note that conjugate involutions can have

\footnotetext{
${ }^{37}$ We list the sizes of the conjugacy classes in Table 22.

${ }^{38}$ In fact, $\operatorname{span}\left\{K, \alpha_{1}, \ldots, \alpha_{n}\right\}$ is a finite-index sublattice of $H_{2}\left(\mathcal{B}_{n}, \mathbb{Z}\right)$ for $n \geq 3$. However, since there is no torsion in the homology of del Pezzo surfaces this does not matter in the following.
} 
Table 22: The $\mathbb{Z}_{2}$ conjugacy classes in the Weyl groups associated to del Pezzo surfaces. These (together with the trivial group element) classify the possible actions on the degree-2 homology of the corresponding del Pezzo surface. Note that in some cases the potential action cannot be realised on a del Pezzo surface. The $d \times d$ identity matrix is denoted by $\mathbf{1}_{d}$, the $2 \times 2$ permutation matrix by $H=\left(\begin{array}{ll}0 & 1 \\ 1 & 0\end{array}\right)$.

\begin{tabular}{|c|c|c|c|c|}
\hline $\begin{array}{l}0 \\
\text { N } \\
0 \\
\text { 2 } \\
\text { 0 }\end{array}$ & 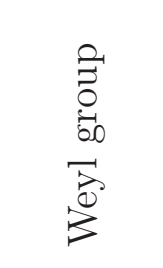 & Action on $H_{2}\left(\mathcal{B}_{n}, \mathbb{Z}\right)=\operatorname{Span}\left\{l, e_{1}, \ldots, e_{n}\right\}$ & $\left(b_{2}^{+}, b_{2}^{-}\right)$ & 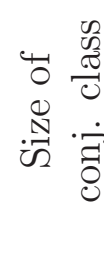 \\
\hline $\mathcal{B}_{3}$ & $A_{2} \oplus A_{1}$ & $\begin{aligned} I_{\mathcal{B}_{3}}^{(1)} & =\mathbf{1}_{2} \oplus H \\
I_{\mathcal{B}_{3}}^{(2)} & =\left(\begin{array}{cccc}2 & 1 & 1 & 1 \\
-1 & -1 & 0 & -1 \\
-1 & 0 & -1 & -1 \\
-1 & -1 & -1 & 0 \\
2 & 1 & 1 \\
-1 & 0 & -1 & 1 \\
-1 & -1 & 0 & -1 \\
-1 & -1 & -1 & 0\end{array}\right) \\
I_{\mathcal{B}_{3}}^{(3)} & =\end{aligned}$ & $\begin{array}{l}(3,1) \\
(2,2) \\
(3,1)\end{array}$ & $\begin{array}{l}3 \\
1\end{array}$ \\
\hline $\mathcal{B}_{4}$ & $A_{4}$ & $\begin{array}{l}I_{\mathcal{B}_{4}}^{(1)}=\mathbf{1}_{3} \oplus H \\
I_{\mathcal{B}_{4}}^{(2)}=\mathbf{1}_{1} \oplus 2 H\end{array}$ & $\begin{array}{l}(4,1) \\
(3,2)\end{array}$ & $\begin{array}{l}10 \\
15\end{array}$ \\
\hline $\mathcal{B}_{5}$ & $D_{5}$ & 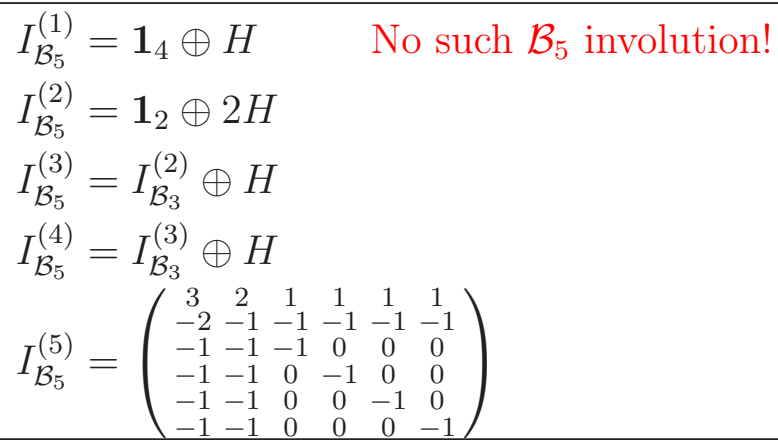 & $\begin{array}{l}(5,1) \\
(4,2) \\
(3,3) \\
(4,2) \\
(2,4)\end{array}$ & $\begin{array}{l}20 \\
60 \\
60 \\
10 \\
5\end{array}$ \\
\hline $\mathcal{B}_{6}$ & $E_{6}$ & 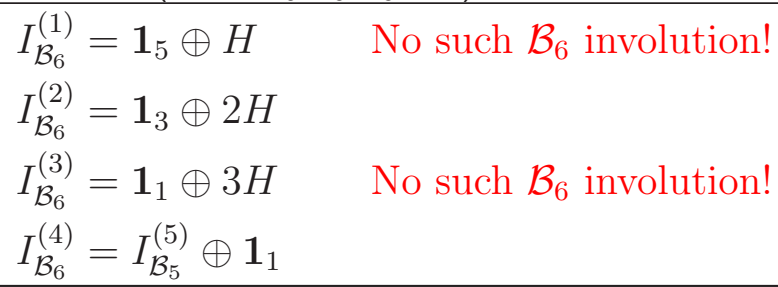 & $\begin{array}{l}(6,1) \\
(5,2) \\
(4,3) \\
(3,4)\end{array}$ & $\begin{array}{c}36 \\
270 \\
540 \\
45\end{array}$ \\
\hline
\end{tabular}

Continued on the next page 
Table 22 - continued from previous page

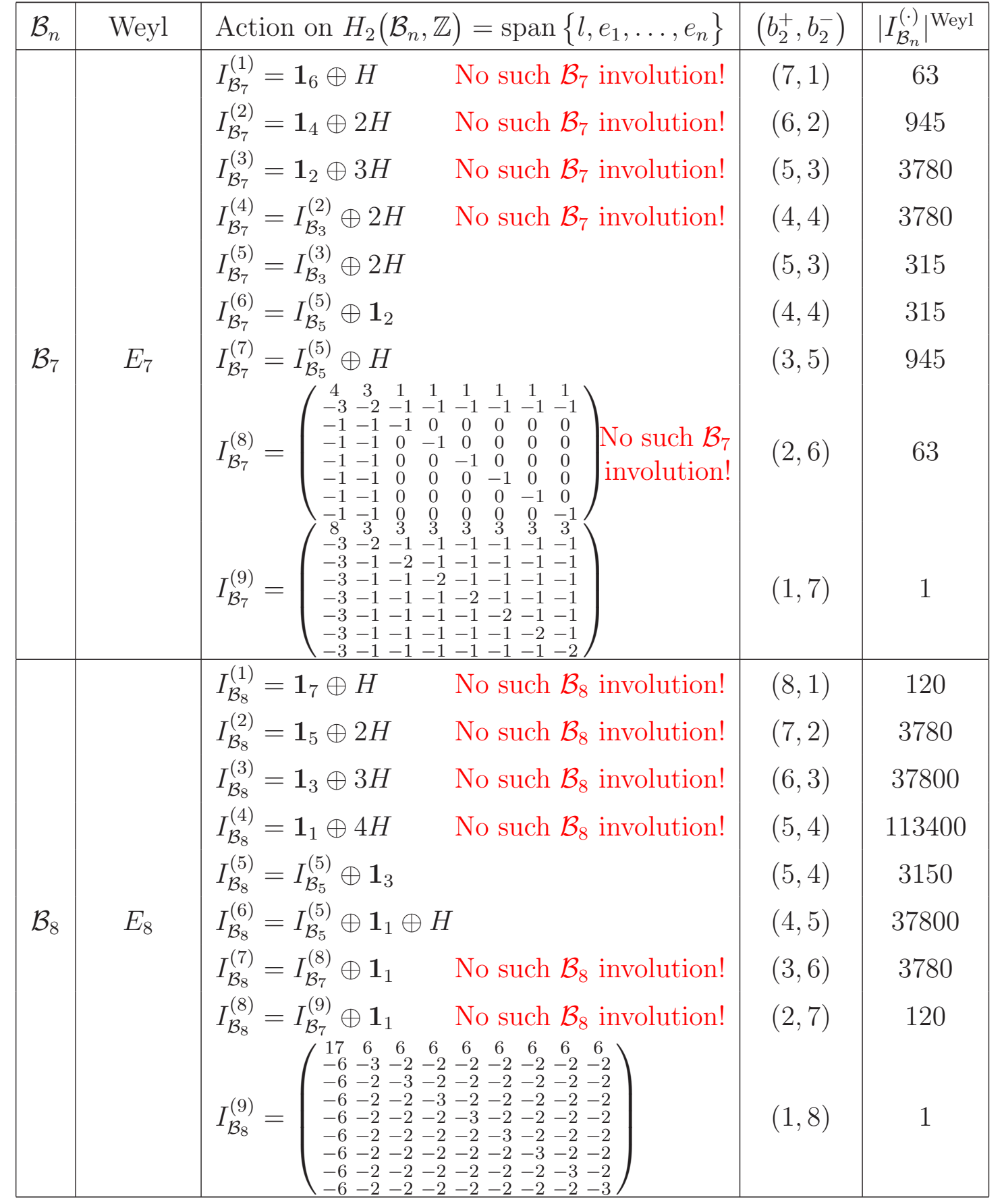


different matrix expressions; We pick particularly "nice" representatives by picking block-diagonal ones such that the bottom right blocks are either $2 \times 2$ permutation matrices, or identity matrices.

Note that no two $(-1)$-curves are homologous, that is, the homology classes of the $(-1)$-curves are all distinct. Hence, the homology class of a $(-1)$-curve can only be invariant under an involution if the $(-1)$-curve is geometrically invariant. Similarly, a pair of homology classes of self-intersection -1 is exchanged under an involution if and only if the actual (-1)-curves are exchanged by the involution. Hence,

- If an involution on $\mathcal{B}_{n}$ leaves one $(-1)$-class invariant, then said involution is the blow-up of an involution on $\mathcal{B}_{n-1}$ at one invariant point. In the standard basis, the group action on $H_{2}\left(\mathcal{B}_{n}, \mathbb{Z}\right)$ is block diagonal, consisting of the action on $H_{2}\left(\mathcal{B}_{n-1}, \mathbb{Z}\right)$ and one $\mathbf{1}_{1}$ block.

- If an involution on $\mathcal{B}_{n}$ exchanges two $(-1)$-classes that do not intersect, then said involution is the blow-up of an involution on $\mathcal{B}_{n-2}$ at a pair of points (that is, a non-fixed point and its image point). In the standard basis, the group action on $H_{2}\left(\mathcal{B}_{n}, \mathbb{Z}\right)$ is block diagonal, consisting of the action on $H_{2}\left(\mathcal{B}_{n-2}, \mathbb{Z}\right)$ and one $H$ block.

\section{A.8 Minimal Involutions}

Analysing the list of possible actions on $H_{2}(S, \mathbb{Z})$ in Table 20 , one easily sees that many are related by adding $\mathbf{1}_{1}$ or $H$ blocks. If the action is induced from a del Pezzo surface, then these operations correspond to blowing up a fixed point and blowing up a point-image point pair, respectively. The minimal group actions, which cannot be built from simpler ones, are

- $\mathbf{1}_{1}$ acting on $H_{2}\left(\mathbb{P}^{2}, \mathbb{Z}\right) \quad$ Realised by $\left(\mathbb{P}^{2}, \sigma\right)$, eq. (231).

- $\mathbf{1}_{2}$ acting on $H_{2}\left(\mathbb{P}^{1} \times \mathbb{P}^{1}, \mathbb{Z}\right)$

Realised by $\left(\mathbb{P}^{1} \times \mathbb{P}^{1}, \sigma_{1}\right)$ and $\left(\mathbb{P}^{1} \times \mathbb{P}^{1}, \sigma_{2}\right)$, eqns. (237a $)$ and (237b).

- $H$ acting on $H_{2}\left(\mathbb{P}^{1} \times \mathbb{P}^{1}, \mathbb{Z}\right)$

Realised by $\left(\mathbb{P}^{1} \times \mathbb{P}^{1}, \sigma_{3}\right)$, eq. (237a).

- $I_{\mathcal{B}_{5}}^{(5)}$ acting on $H_{2}\left(\mathcal{B}_{5}, \mathbb{Z}\right)$

- $I_{\mathcal{B}_{7}}^{(8)}$ acting on $H_{2}\left(\mathcal{B}_{7}, \mathbb{Z}\right)$ 
- $I_{\mathcal{B}_{7}}^{(9)}$ acting on $H_{2}\left(\mathcal{B}_{7}, \mathbb{Z}\right)$

- $I_{\mathcal{B}_{8}}^{(9)}$ acting on $H_{2}\left(\mathcal{B}_{8}, \mathbb{Z}\right)$

Moreover, the first three (and only the first three) are related by adding and subtracting blocks of the form $\mathbf{1}_{1}$ and $H$. In terms of geometric involutions, this means that they are birational. Hence, there are five disconnected cases of involutions modulo blow-up/down. One obvious way to understand these cases is to look at the minimal involution, that is, those that cannot be further blown down. We already analysed the minimal models $\left(\mathbb{P}^{2}, \sigma\right),\left(\mathbb{P}^{1} \times \mathbb{P}^{1}, \sigma_{1}\right),\left(\mathbb{P}^{1} \times \mathbb{P}^{1}, \sigma_{2}\right)$, and $\left(\mathbb{P}^{1} \times \mathbb{P}^{1}, \sigma_{3}\right)$, all of which are birationally equivalent 39 . The remaining four minimal involutions are classically known involutions:

$I_{\mathcal{B}_{8}}^{(9)}$ : The Bertini involution on $\mathcal{B}_{8}$, which we denote by $\left(\mathcal{B}_{8}, \sigma_{\mathrm{B}}\right)$. Its fixed point set see eq. (272), consists of a genus-4 curve and one isolated fixed point. Since $b_{2}^{+}=1$, all invariant homology classes must be proportional to the canonical class. The constant of proportionality determines the genus of a corresponding holomorphic curve via eq. (74). Using this, the homology class of the fixed point set must be

$$
\left[\Sigma_{4}\right]=-3 K=9 l-3 \sum_{i=1}^{8} e_{i} \in H_{2}\left(\mathcal{B}_{8}, \mathbb{Z}\right)^{\sigma_{\mathrm{B}}}=\mathbb{Z} K .
$$

$I_{\mathcal{B}_{7}}^{(9)}$ : The Geiser involution on $\mathcal{B}_{7}$, which we denote by $\left(\mathcal{B}_{7}, \sigma_{\mathrm{G}}\right)$. The fixed point set, see eq. (272), consists of a genus-3 curve with homology class

$$
\left[\Sigma_{3}\right]=-2 K=6 l-2 \sum_{i=1}^{7} e_{i} \in H_{2}\left(\mathcal{B}_{7}, \mathbb{Z}\right)^{\sigma_{\mathrm{G}}}=\mathbb{Z} K .
$$

$I_{\mathcal{B}_{7}}^{(8)}$ : The de Jonquières involution of degree 4 on the blow-up of $\mathbb{P}^{2}$ at 7 points. In this case, 6 of the points necessarily lie on a conic [82, so this involution cannot be realised on a del Pezzo surface. The invariant homology classes are the rank-2 lattice generated by $K$ and $l-e_{1}$. The fixed point set consists of a

\footnotetext{
${ }^{39}$ Evidently, a minimal model can be a "local minimum"
} 
single genus-2 curve $\Sigma_{2}$. Its homology class is

$$
\begin{aligned}
{\left[\Sigma_{2}\right]=-K+\left(l-e_{1}\right)=4 l-2 e_{1} } & -\sum_{i=2}^{7} e_{i} \\
& \in H_{2}\left(\mathcal{B}_{7}, \mathbb{Z}\right)^{\sigma_{\mathrm{dJ}}}=\operatorname{span}_{\mathbb{Z}}\left\{K, l-e_{1}\right\} .
\end{aligned}
$$

$I_{\mathcal{B}_{5}}^{(5)}$ : The de Jonquières involution of degree 3 on $\mathcal{B}_{5}$, which we denote by $\left(\mathcal{B}_{5}, \sigma_{\mathrm{dJ}}\right)$. The fixed point set, see eq. (272), consists of a single genus-1 curve (that is, an elliptic curve) $\Sigma_{1}$ in the class

$$
\left[\Sigma_{1}\right]=-K=3 l-\sum_{i=1}^{5} e_{i} \in H_{2}\left(\mathcal{B}_{5}, \mathbb{Z}\right)^{\sigma_{\mathrm{dJ}}}=\operatorname{span}_{\mathbb{Z}}\left\{K, l-e_{1}\right\}
$$

Each of these involutions comes with moduli, which can be identified with the moduli of the fixed point curve $\Sigma_{g}$ [83,84]. For example, the moduli space of $\left(\mathcal{B}_{5}, \sigma\right)$ is the usual moduli space of elliptic curves 40 . Since these moduli spaces are all connected, we learn that there is (up to continuous deformation) a unique involution giving rise to the actions $\left(I_{\mathcal{B}_{5}}^{(5)}, I_{\mathcal{B}_{7}}^{(8)}, I_{\mathcal{B}_{7}}^{(9)}\right.$, and $I_{\mathcal{B}_{8}}^{(9)}$, respectively) in homology.

Looking at the fixed point sets, we can understand the five different birationally connected components of involutions as follows. Note that blowing up a point on a surface always generates an exceptional divisor $\simeq \mathbb{P}^{1}$ and does not change the genus of other curves. Hence, all fixed points in del Pezzo involutions birationally connected to $\left(\mathbb{P}^{2}, \sigma\right)$ are either isolated fixed points or of genus 0 . The fixed point set in the other 4 disconnected components all contain a single curve of genus $1,2,3$, and 4, respectively. Again, since the genus of these divisors is a birational invariant, these 5 cases cannot be connected by a chain of blow-ups/downs.

\section{A.9 Blow-ups of Minimal Models}

In order to list all involutions on del Pezzo surfaces, we now just have to start with the 5 minimal involutions and perform successive blow-ups at fixed points or pointimage point pairs. Recall that, by definition, the blow-up point must not lie on $(-1)$ curves, see Footnote 17. Together with our local analysis of the fixed-point set after blow-up, Subsection A.4, we can summarise the blow-up procedure as follows:

\footnotetext{
${ }^{40}$ That is, the upper half plane modulo $\operatorname{PSL}(2, \mathbb{Z})$.
} 
- Blowing up an isolated fixed point is only allowed if this fixed point is not part of a $(-1)$-curve. After the blow-up, the isolated fixed point is replaced with the point-wise fixed exceptional divisor $e_{n} \simeq \mathbb{P}^{1}$ on $\mathcal{B}_{n}$. No points of $e_{n}$ may be blown up any further.

- On a point-wise fixed curve $C$ one may blow up points as long as $C^{2} \geq 0$ and the point is not one of the finitely many intersection points with $(-1)$-curves. After the blow up to $\mathcal{B}_{n}$, the fixed curve $C$ is replaced by its proper transform $\tilde{C}$ and one isolated point $\tilde{p}$. The new exceptional divisor $e_{n}$ is not point-wise fixed, but intersects $\tilde{C} \cdot e_{n}=1$ and contains $\tilde{p} \in e_{n}$. In particular, $\tilde{p}$ may not be blown up further. The homology class of the new fixed-point set is

$$
[\tilde{C}]=\left[C-e_{n}\right] \in H_{2}\left(\mathcal{B}_{n}, \mathbb{Z}\right)
$$

and $\tilde{C}^{2}=C^{2}-1$.

- A generic point $p$ is neither fixed nor part of the finitely many (-1)-curves. Hence, one can always find such a point $p$ and image point $q$. However, since the two points are not independent, one must check that all points are still in general position, see Footnote 17. The fixed point set does not change when blowing up such a pair of points.

Clearly, the important data about the fixed points set are the curves and the isolated fixed points that can/cannot be blown up further.

The successive blow-ups of minimal involutions are then subject to the requirement that the blow-up points are in general position. This yields the following restrictions:

- Consider a generic point-image point pair on $\left(\mathbb{P}^{2}, \sigma\right)$,

$$
p=\left[y: x_{0}: x_{1}\right], \quad q=\left[-y, x_{0}: x_{1}\right] \in \mathbb{P}^{2} .
$$

$p, q$, and the isolated fixed point $[1: 0: 0] \in\left(\mathbb{P}^{2}\right)^{\sigma}$ are collinear. Hence, one may either blow up this isolated fixed point or blow up a point-image point pair, but not all three.

- Amongst 4 invariant points on $\left(\mathbb{P}^{2}, \sigma\right)$ there are 3 collinear ones. This excludes actions containing $\mathbf{1}_{5}$ and higher. 
- Any 3 point-image point pairs on $\left(\mathbb{P}^{2}, \sigma\right)$ lie on a conic. This excludes actions containing $3 H$ and $4 H$.

- Any 3 point-image point pairs on $\left(\mathbb{P}^{1} \times \mathbb{P}^{1}, \sigma_{3}\right)$ lie on a conic. This excludes the action $I_{\mathcal{B}_{3}}^{(2)} \oplus 2 H$.

- The de Jonquières involution of degree 4 on the blow-up of $\mathbb{P}^{2}$ at 7 points is excluded since there are 6 points lying on a conic.

- Recall the action of the Geiser involution on $\mathcal{B}_{7}=\mathrm{Bl}_{p_{1}, \ldots, p_{7}} \mathbb{P}^{2}$, the image of a point $q \in \mathcal{B}_{7}$ is the remaining basepoint of the pencil of cubics going through the 8 points $\left\{p_{1}, \ldots, p_{7}, q\right\}$. If this remaining basepoint coincides with $q$, then the cubic has a node at $q$. Hence, blowing up a fixed point of $\left(\mathcal{B}_{7}, \sigma_{\mathrm{G}}\right)$ is excluded as it would result in a nodal cubic going through the 8 points.

Starting from the minimal involutions, this lets us enumerate all involutions on del Pezzo surfaces. The result is presented in Table 6. These involutions are related through blowing up fixed points or point-image point pairs. This is depicted in Figure 10.

\section{A.10 Explicit Realisations}

Finally, let us list explicit examples for the involutions on del Pezzo surfaces of degree 5 and less. These can be written as hypersurfaces in weighted projective spaces. For simplicity, we pick a particular simple point in the complex moduli space in each case:

$$
\begin{array}{ll}
\mathcal{B}_{6}=\left\{x_{0}^{3}+x_{1}^{3}+x_{2}^{3}+x_{3}^{3}=0\right\} & \subset \mathbb{P}^{1,1,1,1} \\
\mathcal{B}_{7}=\left\{y^{2}+x_{0}^{4}+x_{1}^{4}+x_{2}^{4}=0\right\} & \subset \mathbb{P}^{2,1,1,1} \\
\mathcal{B}_{7}=\left\{y^{2}+z^{3}+x_{0}^{6}+x_{1}^{6}=0\right\} & \subset \mathbb{P}^{3,2,1,1}
\end{array}
$$



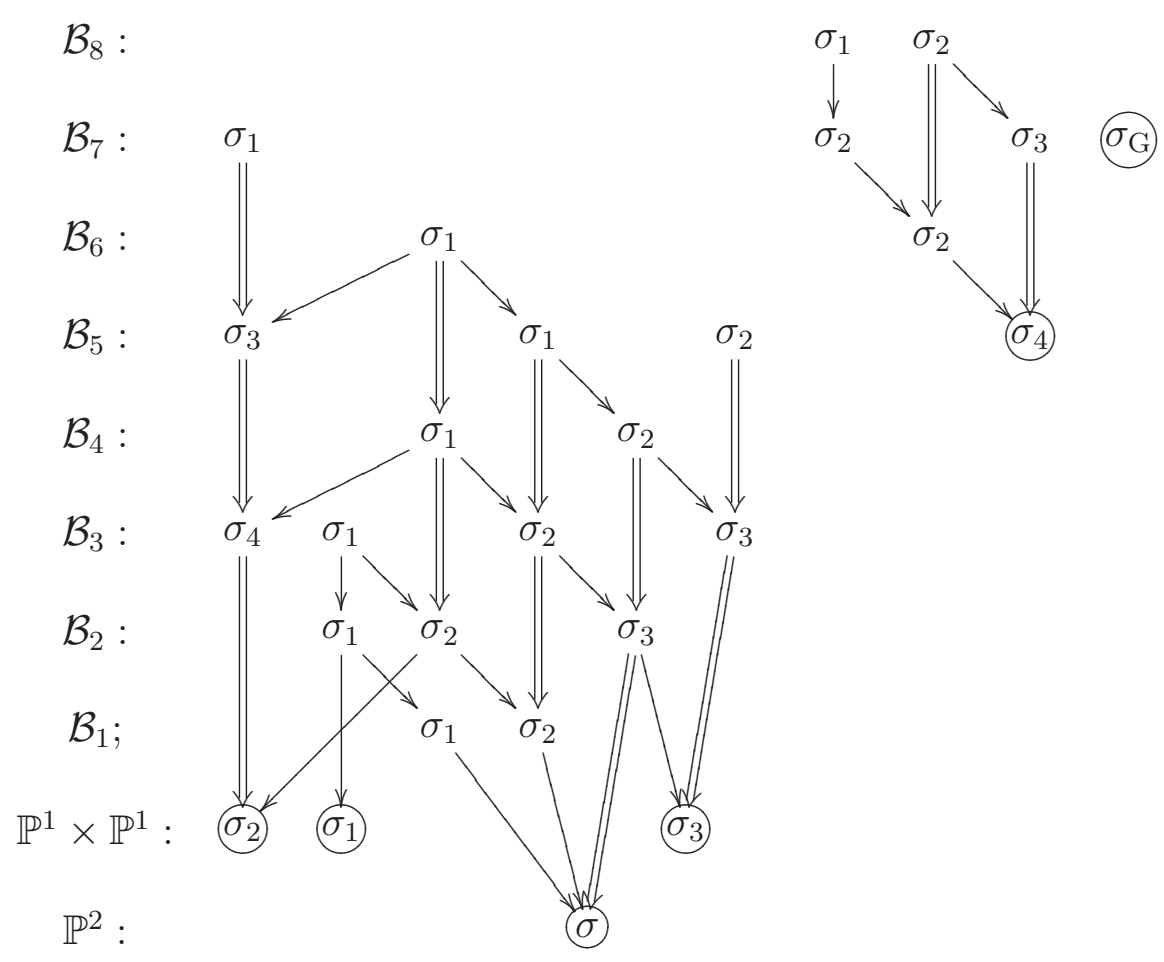

$\sigma_{\mathrm{B}}$

$\sigma_{G}$

Figure 10: Relations between the involutions on del Pezzo surfaces. The single arrows $(\rightarrow)$ are the blow-downs of a fixed $(-1)$-curve, the double arrows $(\Rightarrow)$ are the blow-downs of a $(-1)$-curve and its image curve. The minimal involutions are encircled. 
The involutions listed in Table 6 then act as follows on the hypersurfaces:

$$
\begin{aligned}
& \sigma_{1}: \mathcal{B}_{6} \rightarrow \mathcal{B}_{6}, \quad\left[x_{0}: x_{1}: x_{2}: x_{3}\right] \mapsto\left[x_{1}: x_{0}: x_{3}: x_{2}\right], \\
& \sigma_{2}: \mathcal{B}_{6} \rightarrow \mathcal{B}_{6}, \quad\left[x_{0}: x_{1}: x_{2}: x_{3}\right] \mapsto\left[x_{1}: x_{0}: x_{2}: x_{3}\right], \\
& \sigma_{1}: \mathcal{B}_{7} \rightarrow \mathcal{B}_{7}, \quad\left[y: x_{0}: x_{1}: x_{2}\right] \mapsto\left[-y:-x_{0}: x_{1}: x_{2}\right], \\
& \sigma_{2}: \mathcal{B}_{7} \rightarrow \mathcal{B}_{7}, \quad\left[y: x_{0}: x_{1}: x_{2}\right] \mapsto\left[y:-x_{0}: x_{1}: x_{2}\right], \\
& \sigma_{3}: \mathcal{B}_{7} \rightarrow \mathcal{B}_{7}, \quad\left[y: x_{0}: x_{1}: x_{2}\right] \mapsto\left[y: x_{1}: x_{0}: x_{2}\right], \\
& \sigma_{\mathrm{G}}: \mathcal{B}_{7} \rightarrow \mathcal{B}_{7}, \quad\left[y: x_{0}: x_{1}: x_{2}\right] \mapsto\left[-y: x_{0}: x_{1}: x_{2}\right], \\
& \sigma_{1}: \mathcal{B}_{8} \rightarrow \mathcal{B}_{8}, \quad\left[y: z: x_{0}: x_{1}\right] \mapsto\left[y: z:-x_{0}: x_{1}\right], \\
& \sigma_{2}: \mathcal{B}_{8} \rightarrow \mathcal{B}_{8}, \quad\left[y: z: x_{0}: x_{1}\right] \mapsto\left[y: z: x_{1}: x_{0}\right], \\
& \sigma_{\mathrm{B}}: \mathcal{B}_{8} \rightarrow \mathcal{B}_{8}, \quad\left[y: z: x_{0}: x_{1}\right] \mapsto\left[-y: z: x_{0}: x_{1}\right] .
\end{aligned}
$$

\section{B Cohomology of Line Bundles over del Pezzo Surfaces}

On a del Pezzo surface $\mathcal{B}_{n}=\mathrm{Bl}_{p_{1}, p_{2}, \ldots, p_{n}}\left(\mathbb{P}^{2}\right), n \leq 8$, the line bundles 41

$$
\operatorname{Pic}\left(\mathcal{B}_{n}\right)=H^{2}\left(\mathcal{B}_{n}, \mathbb{Z}\right)=\operatorname{span}_{\mathbb{Z}}\left\{l, e_{1}, e_{2}, \ldots, e_{n}\right\} \simeq \mathbb{Z}^{n+1}
$$

are classified by their first Chern class. We will parametrise the first Chern class of any line bundle $L$ as

$$
L=\mathcal{O}\left(a l+\sum_{i \in I} b_{i} e_{i}-\sum_{j \in J} c_{j} e_{j}\right), \quad a \in \mathbb{Z}, b_{i} \in \mathbb{Z}_{\geq}, c_{j} \in \mathbb{Z}_{>},
$$

where we split the index range $\{1,2, \ldots, n\}=I \cup J$ into two disjoint index sets.

In order to compute the bundle cohomology groups of $L$, we fist recall the following two facts:

- The index of $L$ is

$$
\begin{aligned}
\chi(L) & =\sum_{k=0}^{2}(-1)^{k} h^{k}\left(\mathcal{B}_{n}, L\right)=\int_{\mathcal{B}_{n}} \operatorname{ch}(L) \operatorname{Td}\left(T \mathcal{B}_{n}\right)= \\
& =\left(\begin{array}{c}
a+2 \\
2
\end{array}\right)-\sum_{i \in I} \frac{b_{i}\left(b_{i}-1\right)}{2}-\sum_{j \in J} \frac{c_{j}\left(c_{j}+1\right)}{2} .
\end{aligned}
$$

\footnotetext{
${ }^{41}$ We are going to label the exceptional divisors such that $e_{k} \subset \mathcal{B}_{n}$ corresponds to the blow-up point $p_{k} \in \mathbb{P}^{2}, k=1, \ldots, n$.
} 
- Serre duality relates

$$
\begin{aligned}
H^{k}\left(\mathcal{B}_{n}, L\right)^{\vee} & =H^{2-k}\left(\mathcal{B}_{n}, L^{\vee} \otimes K\right) \\
& =H^{2-k}\left(\mathcal{B}_{n}, L^{\vee} \otimes \mathcal{O}\left(-3 l+e_{1}+\cdots+e_{n}\right)\right)
\end{aligned}
$$

Using Serre duality if necessary, it therefore suffices to calculate the cohomology groups of $L$ for $a \geq-2$. In the following, we will always assume this to be the case.

First, the cohomology of $\mathcal{O}(a l)$ is clearly identical to the cohomology of $\mathcal{O}_{\mathbb{P}^{2}}(a)$, which is

$$
H^{*}\left(\mathcal{B}_{n}, \mathcal{O}(a l)\right)= \begin{cases}0 & *=2 \\
0 & *=1 \\
\left(\begin{array}{c}
a+2 \\
2
\end{array}\right) & *=0\end{cases}
$$

The $\left(\begin{array}{c}a+2 \\ 2\end{array}\right)$ global sections are nothing but the degree- $a$ homogeneous polynomials in the 3 homogeneous variables. Similarly, the global sections of $\mathcal{O}\left(a l-\sum c_{i} e_{i}\right)$ can be identified with the degree- $a$ homogeneous polynomials that vanish at the blow-up point $p_{j}$ to the degree $c_{j}, j \in J$. Note that the homogeneous polynomials are a linear space spanned by the monomials, and, therefore, counting the dimension of the space of such sections is a simple linear algebra problem. We denote the dimension of the sections vanishing at $p_{1}, \ldots, p_{n}$ by 42

$$
A_{\sum c_{i} p_{i}}(a)=\operatorname{dim}\left\{P_{a}(x, y, z) \mid P_{a}\left(p_{i}\right)=0 \text { to order } c_{i}\right\} .
$$

To obtain the higher-degree cohomology groups, consider the standard short exact sequence

$$
0 \longrightarrow \mathcal{O}_{\mathcal{B}_{n}}\left(a l-\sum c_{j} e_{j}\right) \longrightarrow \mathcal{O}_{\mathcal{B}_{n}}(a l) \longrightarrow \oplus_{j \in J} \mathcal{O}_{c_{j} e_{j}} \longrightarrow 0
$$

${ }^{42}$ The expected dimension is

$$
A_{\sum c_{i} p_{i}}^{\text {expect }}(a)=\max \left\{0,\left(\begin{array}{c}
a+2 \\
2
\end{array}\right)-\sum \frac{c_{j}\left(c_{j}+1\right)}{2}\right\},
$$

and this is often the actual value. However, for example, $\mathcal{O}_{\mathcal{B}_{3}}\left(3-3 e_{1}-2 e_{2}-e_{3}\right)$ has $A_{3 e_{1}+2 e_{2}+e_{1}}^{\text {expect }}(3)=$ 0 while the actual value is $A_{3 e_{1}+2 e_{2}+e_{1}}(3)=1$. Moreover, for special values of the complex structure moduli of $\mathcal{B}_{n}$, the dimension of the cohomology group jumps and $A_{\sum c_{i} p_{i}}(a)>A_{\sum c_{i} p_{i}}^{\text {expect }}(a)$. Note that one can always pick coordinates on $\mathbb{P}^{2}$ such that

$$
p_{1}=[1: 0: 0], p_{2}=[0: 1: 0], p_{3}=[0: 0: 1], p_{4}=[1: 1: 1] .
$$

In other words, only $\mathcal{B}_{n}$ with $n \geq 5$ have complex structure moduli, parametrised by the position of the points $p_{5}, \ldots, p_{n}$. 
From the corresponding long exact sequence 43 ,

$$
\begin{aligned}
& 0 \longrightarrow H^{0}\left(\mathcal{O}_{\mathcal{B}_{n}}\left(a l-\sum c_{j} e_{j}\right)\right) \longrightarrow\left(\begin{array}{c}
a+2 \\
2
\end{array}\right) \longrightarrow \sum \frac{c_{j}\left(c_{j}+1\right)}{2} \longrightarrow \\
& \longrightarrow H^{1}\left(\mathcal{O}_{\mathcal{B}_{n}}\left(a l-\sum c_{j} e_{j}\right)\right) \longrightarrow 0
\end{aligned}
$$

it follows that the cohomology is concentrated in degrees 0 and 1 only. Hence, using the index, one obtains that

$$
H^{2}\left(\mathcal{B}_{n}, \mathcal{O}\left(a l-\sum c_{j} e_{j}\right)\right)=0
$$

Finally, consider the short exact sequence

$$
0 \longrightarrow \mathcal{O}_{\mathcal{B}_{n}}\left(a l-\sum c_{j} e_{j}\right) \longrightarrow L \longrightarrow \oplus_{i \in I} \mathcal{O}_{b_{i} e_{i}}\left(-b_{i}\right) \longrightarrow 0
$$

The corresponding long exact sequence reads

$$
\begin{array}{r}
0 \longrightarrow H^{0}\left(\mathcal{O}_{\mathcal{B}_{n}}\left(a l-\sum c_{i} e_{i}\right)\right) \longrightarrow H^{0}(L) \longrightarrow H^{1}\left(\mathcal{O}_{\mathcal{B}_{n}}\left(a l-\sum c_{i} e_{i}\right)\right) \longrightarrow H^{1}(L) \longrightarrow \sum \frac{b_{i}\left(b_{i}+1\right)}{2} \longrightarrow \\
\longrightarrow 0 \longrightarrow H^{2}(L) \longrightarrow 0 \\
\longrightarrow 0 .
\end{array}
$$

One immediately notices that the cohomology of $L$ is concentrated in degrees 0 and 1 , and therefore is determined by the index and $A$. Therefore, the cohomology of $L=\mathcal{O}\left(a l+\sum b_{i} e_{i}-\sum c_{j} e_{j}\right)$ with $a \geq-2$ is given by

$$
H^{*}\left(\mathcal{B}_{n}, L\right)= \begin{cases}0 & *=2 \\ -\chi(L)-A_{\sum c_{i} p_{i}}(a) & *=1 \\ A_{\sum c_{i} p_{i}}(a) & *=0 .\end{cases}
$$

\footnotetext{
${ }^{43} \mathrm{By}$ abuse of notation, we just write $k$ instead of a $k$-dimensional vector space in long exact sequences.
} 


\section{Cohomology of Line Bundles On Rational El- liptic Surfaces}

By definition, a $\mathrm{dP}_{9}$ surface is a rational elliptic surface, meaning that it is simultaneously a blow-up of $\mathbb{P}^{2}$ and elliptically fibred. Given this definition, one can show that there must be precisely 9 blow-up point 44

$$
\mathrm{dP}_{9}=\mathrm{Bl}_{\left\{p_{1}, \ldots, p_{9}\right\}}\left(\mathbb{P}^{2}\right),
$$

that the base of the fibration must be $\mathbb{P}^{1}$ and can 45 be taken to be $e_{9}$ ), and that the fibre class is

$$
f=3 l-\sum_{i=1}^{9} e_{i} \in H_{2}\left(\mathrm{dP}_{9}, \mathbb{Z}\right) .
$$

However, the position of the 9 points are not arbitrary. In other words, not every blow-up of $\mathbb{P}^{2}$ at 9 points is elliptically fibred, but, rather, the blow-up points have to be in the right position. The immediate consequence for line bundles is that often the actual cohomology groups are larger than what one would obtain from the naive application of Appendix B. For example [85],

$$
H^{*}\left(\mathrm{dP}_{9}, \mathcal{O}_{\mathrm{dP}_{9}}(f)\right)=(2,1,0),
$$

while eq. (286) would have yielded $(1,0,0)$.

However, there are two special case 47 where we can, in fact, simply apply the results of Appendix B for $\mathcal{B}_{r}, r=0, \ldots, 8$ :

- Consider the case where one of the exceptional divisors (say, $e_{9}$ ) does not appear in the line bundle, that is,

$$
L=\mathcal{O}\left(a l+\sum_{i \in I} b_{i} e_{i}-\sum_{j \in J} c_{j} e_{j}+0 \cdot e_{9}\right), \quad a \in \mathbb{Z}, b_{i} \in \mathbb{Z}_{\geq}, c_{j} \in \mathbb{Z}_{>},
$$

\footnotetext{
${ }^{44}$ In contrast to the del Pezzo case, the blow-up points can be "infinitesimally close". This generates (-2)-curves in the blown-up surface, which are allowed on a $\mathrm{dP}_{9}$ but not on a del Pezzo surface. In fact, the irreducible components of most Kodaira fibres (all except $I_{0}, I_{1}$, and $I I I$ ) have self-intersection -2 .

${ }^{45}$ And we will make this choice always in the following.

${ }^{46}$ Of course, the index is the same since it is a topological quantity.

${ }^{47}$ Here, and in the following, we will assume that we are on a sufficiently generic $\mathrm{dP}_{9}$ surface where all blow-up points $p_{1}, \ldots, p_{9}$ can be chosen to be distinct.
} 
with $I \cup J=\{1, \ldots, 8\}$ disjoint index sets. Then, we can identify the cohomology with the analogue

$$
H^{*}\left(\mathrm{dP}_{9}, L\right)=H^{*}\left(\mathcal{B}_{8}, \mathcal{O}_{\mathcal{B}_{8}}\left(a l+\sum_{i \in I} b_{i} e_{i}-\sum_{j \in J} c_{j} e_{j}\right)\right)
$$

on $\mathcal{B}_{8}$, where eq. (286) holds.

- The canonical bundle is

$$
K_{\mathrm{dP}_{9}}=\mathcal{O}_{\mathrm{dP}_{9}}(-f),
$$

and, therefore, Serre duality allows us to identify

$$
H^{*}\left(\mathrm{dP}_{9}, L \otimes \mathcal{O}_{\mathrm{dP}_{9}}(-f)\right)=H^{2-*}\left(\mathrm{dP}_{9}, L^{\vee}\right)^{\vee} .
$$

However, in general one has to use the use the program detailed in [85], and use the Leray-Serre spectral sequence for the elliptic fibration $\pi: \mathrm{dP}_{9} \rightarrow \mathbb{P}^{1}$. This revolves around the three steps:

1. Compute the push-down $R^{q} \pi_{*} L$ for linear combinations of sections, adding and subtracting one section $s$ at a time. This amounts to repeated applications of the short exact sequence

$$
0 \longrightarrow \mathcal{O}_{\mathrm{dP}_{9}}(D-s) \longrightarrow \mathcal{O}_{\mathrm{dP}_{9}}(D) \longrightarrow \mathcal{O}_{s}(D \cdot s) \longrightarrow 0
$$

and the corresponding long exact sequence

$$
\begin{gathered}
0 \longrightarrow \pi_{*}\left(\mathcal{O}_{\mathrm{dP}_{9}}(D-s)\right) \longrightarrow \pi_{*}\left(\mathcal{O}_{\mathrm{dP}_{9}}(D)\right) \longrightarrow \mathcal{O}_{\mathbb{P}^{1}}(D \cdot s) \longrightarrow \\
\longrightarrow R^{1} \pi_{*}\left(\mathcal{O}_{\mathrm{dP}_{9}}(D-s)\right) \longrightarrow R^{1} \pi_{*}\left(\mathcal{O}_{\mathrm{dP}_{9}}(D)\right) \longrightarrow 0
\end{gathered}
$$

for push-downs.

2. Use the projection formula

$$
R^{q} \pi_{*}\left(L \otimes \mathcal{O}_{\mathrm{dP}_{9}}(n f)\right)=R^{q} \pi_{*}(L) \otimes \mathcal{O}_{\mathbb{P}^{1}}(n)
$$

to shift fibre classes to the base $\mathbb{P}^{1}$. 
3. Compute the cohomology groups of $R^{q} \pi_{*}(L)$ (on $\mathbb{P}^{1}$ ) and use the Leray-Serre spectral sequence to conclude

$$
H^{*}\left(\mathrm{dP}_{9}, L\right)= \begin{cases}H^{1}\left(\mathbb{P}^{1}, R^{1} \pi_{*}(L)\right) & *=2 \\ H^{1}\left(\mathbb{P}^{1}, \pi_{*}(L)\right) \oplus H^{0}\left(\mathbb{P}^{1}, R^{1} \pi_{*}(L)\right) & *=1 \\ H^{0}\left(\mathbb{P}^{1}, \pi_{*}(L)\right) & *=0\end{cases}
$$

As an example, let us consider $L=\mathcal{L}^{-2} \otimes \mathcal{L}_{Y}^{-1}=\mathcal{O}_{\mathrm{dP}_{9}}\left(2 e_{9}+e_{1}-e_{2}-2 f\right)$ in Table 8. Adding and subtracting sections yields (vanishing $R^{1} \pi_{*}$ s are skipped)

$$
\begin{aligned}
\pi_{*}\left(\mathcal{O}_{\mathrm{dP}_{9}}\right) & =\mathcal{O}_{\mathbb{P}^{1}}, \quad R^{1} \pi_{*}\left(\mathcal{O}_{\mathrm{dP}_{9}}\right)=\mathcal{O}_{\mathbb{P}^{1}}(-1), \\
\pi_{*}\left(\mathcal{O}_{\mathrm{dP}_{9}}\left(e_{9}\right)\right) & =\mathcal{O}_{\mathbb{P}^{1}} \\
\pi_{*}\left(\mathcal{O}_{\mathrm{dP}_{9}}\left(2 e_{9}\right)\right) & =\mathcal{O}_{\mathbb{P}^{1}} \oplus \mathcal{O}_{\mathbb{P}^{1}}(-2), \\
\pi_{*}\left(\mathcal{O}_{\mathrm{dP}_{9}}\left(2 e_{9}+e_{1}\right)\right) & =\mathcal{O}_{\mathbb{P}^{1}} \oplus \mathcal{O}_{\mathbb{P}^{1}}(-2) \oplus \mathcal{O}_{\mathbb{P}^{1}}(-1), \\
\pi_{*}\left(\mathcal{O}_{\mathrm{dP}_{9}}\left(2 e_{9}+e_{1}-e_{2}\right)\right) & =\mathcal{O}_{\mathbb{P}^{1}}(-2) \oplus \mathcal{O}_{\mathbb{P}^{1}}(-1) .
\end{aligned}
$$

Hence, using eq. (296),

$$
\begin{aligned}
\pi_{*}(L) & =\mathcal{O}_{\mathbb{P}^{1}}(-4) \oplus \mathcal{O}_{\mathbb{P}^{1}}(-3) \\
R^{1} \pi_{*}(L) & =0 .
\end{aligned}
$$

Finally, the Leray-Serre spectral sequence yields

$$
H^{*}\left(\mathrm{dP}_{9}, L\right)=(0,5,0) .
$$

\section{Bibliography}

[1] R. Blumenhagen, M. Cvetič, P. Langacker, and G. Shiu, "Toward realistic intersecting D-brane models," Ann. Rev. Nucl. Part. Sci. 55 (2005) 71-139, hep-th/0502005.

[2] M. R. Douglas and S. Kachru, "Flux compactification," Rev. Mod. Phys. 79 (2007) 733-796, hep-th/0610102.

[3] R. Blumenhagen, B. Kors, D. Lüst, and S. Stieberger, "Four-dimensional String Compactifications with D-Branes, Orientifolds and Fluxes," Phys. Rept. 445 (2007) 1-193, hep-th/0610327. 
[4] F. Denef, M. R. Douglas, and S. Kachru, "Physics of string flux compactifications," Ann. Rev. Nucl. Part. Sci. 57 (2007) 119-144, hep-th/0701050.

[5] H. P. Nilles, S. Ramos-Sanchez, M. Ratz, and P. K. S. Vaudrevange, "From strings to the MSSM," 0806.3905.

[6] S. Kachru, R. Kallosh, A. Linde, and S. P. Trivedi, "De Sitter vacua in string theory," Phys. Rev. D68 (2003) 046005, hep-th/0301240

[7] V. Balasubramanian, P. Berglund, J. P. Conlon, and F. Quevedo, "Systematics of moduli stabilisation in Calabi-Yau flux compactifications," JHEP 03 (2005) 007, hep-th/0502058.

[8] J. P. Conlon, F. Quevedo, and K. Suruliz, "Large-volume flux compactifications: Moduli spectrum and D3/D7 soft supersymmetry breaking," JHEP 08 (2005) 007, hep-th/0505076.

[9] L. Aparicio, D. G. Cerdeno, and L. E. Ibanez, "Modulus-dominated SUSY-breaking soft terms in F-theory and their test at LHC," JHEP 07 (2008) 099, 0805.2943.

[10] R. Blumenhagen, S. Moster, and E. Plauschinn, "String GUT Scenarios with Stabilised Moduli," 0806.2667.

[11] R. Blumenhagen, B. Körs, D. Lüst, and T. Ott, "The standard model from stable intersecting brane world orbifolds," Nucl. Phys. B616 (2001) 3-33, hep-th/0107138.

[12] C. Vafa, "Evidence for F-Theory," Nucl. Phys. B469 (1996) 403-418, hep-th/9602022.

[13] R. Donagi and M. Wijnholt, "Model Building with F-Theory," 0802.2969.

[14] C. Beasley, J. J. Heckman, and C. Vafa, "GUTs and Exceptional Branes in F-theory - I," 0802.3391.

[15] H. Hayashi, R. Tatar, Y. Toda, T. Watari, and M. Yamazaki, "New Aspects of Heterotic-F Theory Duality," 0805.1057.

[16] C. Beasley, J. J. Heckman, and C. Vafa, "GUTs and Exceptional Branes in F-theory - II: Experimental Predictions," 0806.0102. 
[17] R. Donagi and M. Wijnholt, "Breaking GUT Groups in F-Theory," 0808.2223 .

[18] A. Font and L. E. Ibanez, "Yukawa Structure from U(1) Fluxes in F-theory Grand Unification," 0811.2157.

[19] J. J. Heckman and C. Vafa, "Flavor Hierarchy From F-theory," 0811.2417.

[20] D. Lüst, P. Mayr, S. Reffert, and S. Stieberger, "F-theory flux, destabilization of orientifolds and soft terms on D7-branes," Nucl. Phys. B732 (2006) 243-290, hep-th/0501139.

[21] P. Aluffi and M. Esole, "Chern class identities from tadpole matching in type IIB and F-theory," 0710.2544.

[22] A. P. Braun, A. Hebecker, and H. Triendl, "D7-Brane Motion from M-Theory Cycles and Obstructions in the Weak Coupling Limit," Nucl. Phys. B800 (2008) 298-329, 0801.2163.

[23] A. Collinucci, F. Denef, and M. Esole, "D-brane Deconstructions in IIB Orientifolds," 0805.1573.

[24] A. P. Braun, A. Hebecker, C. Ludeling, and R. Valandro, "Fixing D7 Brane Positions by F-Theory Fluxes," 0811.2416.

[25] R. Blumenhagen, M. Cvetič, and T. Weigand, "Spacetime instanton corrections in $4 \mathrm{D}$ string vacua - the seesaw mechanism for D-brane models," Nucl. Phys. B771 (2007) 113-142, hep-th/0609191.

[26] L. E. Ibanez and A. M. Uranga, "Neutrino Majorana masses from string theory instanton effects," JHEP 03 (2007) 052, hep-th/0609213.

[27] B. Florea, S. Kachru, J. McGreevy, and N. Saulina, "Stringy instantons and quiver gauge theories," JHEP 05 (2007) 024, hep-th/0610003.

[28] R. Blumenhagen, M. Cvetič, D. Lüst, R. Richter, and T. Weigand, "Non-perturbative Yukawa Couplings from String Instantons," Phys. Rev. Lett. 100 (2008) 061602, 0707.1871.

[29] H. Verlinde and M. Wijnholt, "Building the standard model on a D3-brane," JHEP 01 (2007) 106, hep-th/0508089. 
[30] M. Buican, D. Malyshev, D. R. Morrison, H. Verlinde, and M. Wijnholt, "D-branes at singularities, compactification, and hypercharge," JHEP 01 (2007) 107, hep-th/0610007.

[31] E. Witten, "New Issues in Manifolds of SU(3) Holonomy," Nucl. Phys. B268 (1986) 79.

[32] R. Blumenhagen, G. Honecker, and T. Weigand, "Loop-corrected compactifications of the heterotic string with line bundles," JHEP 06 (2005) 020, hep-th/0504232.

[33] R. Tatar and T. Watari, "Proton decay, Yukawa couplings and underlying gauge symmetry in string theory," Nucl. Phys. B747 (2006) 212-265, hep-th/0602238.

[34] R. Blumenhagen, S. Moster, and T. Weigand, "Heterotic GUT and standard model vacua from simply connected Calabi-Yau manifolds," Nucl. Phys. B751 (2006) 186-221, hep-th/0603015.

[35] R. Blumenhagen, S. Moster, R. Reinbacher, and T. Weigand, "Massless spectra of three generation U(N) heterotic string vacua," JHEP 05 (2007) 041, hep-th/0612039.

[36] R. Tatar and T. Watari, "GUT Relations from String Theory Compactifications," 0806.0634.

[37] J. J. Heckman, J. Marsano, N. Saulina, S. Schafer-Nameki, and C. Vafa, "Instantons and SUSY breaking in F-theory," 0808.1286.

[38] J. Marsano, N. Saulina, and S. Schafer-Nameki, "An Instanton Toolbox for F-Theory Model Building," 0808.2450.

[39] J. Marsano, N. Saulina, and S. Schafer-Nameki, "Gauge Mediation in F-Theory GUT Models," 0808.1571.

[40] E. Witten, "PiTP Lecture: String Compactifications I," http://video.ias.edu/PiTP-2008-Witten1.

[41] T. W. Grimm and A. Klemm, "U(1) Mediation of Flux Supersymmetry Breaking," JHEP 10 (2008) 077, 0805.3361. 
[42] D. S. Freed and E. Witten, "Anomalies in string theory with D-branes," hep-th/9907189.

[43] I. Antoniadis, E. Kiritsis, and T. N. Tomaras, "A D-brane alternative to unification," Phys. Lett. B486 (2000) 186-193, hep-ph/0004214.

[44] J. R. Ellis, P. Kanti, and D. V. Nanopoulos, "Intersecting branes flip SU(5)," Nucl. Phys. B647 (2002) 235-251, hep-th/0206087.

[45] M. Cvetič, I. Papadimitriou, and G. Shiu, "Supersymmetric three family $\mathrm{SU}(5)$ grand unified models from type IIA orientifolds with intersecting D6-branes," Nucl. Phys. B659 (2003) 193-223, hep-th/0212177.

[46] F. Gmeiner and M. Stein, "Statistics of SU(5) D-brane models on a type II orientifold," Phys. Rev. D73 (2006) 126008, hep-th/0603019.

[47] M. Cvetič and P. Langacker, "New grand unified models with intersecting D6-branes, neutrino masses, and flipped SU(5)," Nucl. Phys. B776 (2007) 118-137, hep-th/0607238.

[48] I. Antoniadis, A. Kumar, and B. Panda, "Supersymmetric SU(5) GUT with Stabilized Moduli," Nucl. Phys. B795 (2008) 69-104, 0709.2799.

[49] T. W. Grimm and J. Louis, "The effective action of $\mathrm{N}=1$ Calabi-Yau orientifolds," Nucl. Phys. B699 (2004) 387-426, hep-th/0403067.

[50] T. W. Grimm, "The effective action of type II Calabi-Yau orientifolds," Fortsch. Phys. 53 (2005) 1179-1271, hep-th/0507153.

[51] M. Marino, R. Minasian, G. W. Moore, and A. Strominger, "Nonlinear instantons from supersymmetric p-branes," JHEP 01 (2000) 005, hep-th/9911206.

[52] R. Blumenhagen, G. Honecker, and T. Weigand, "Supersymmetric (non-)abelian bundles in the type I and SO(32) heterotic string," JHEP 08 (2005) 009, hep-th/0507041.

[53] R. Blumenhagen, G. Honecker, and T. Weigand, "Non-abelian brane worlds: The open string story," hep-th/0510050.

[54] E. Plauschinn, "The generalized Green-Schwarz mechanism for Type IIB orientifolds with D3- and D7-branes," 0811.2804. 
[55] H. Jockers and J. Louis, "The effective action of D7-branes in $\mathrm{N}=1$ Calabi-Yau orientifolds," Nucl. Phys. B705 (2005) 167-211, hep-th/0409098.

[56] H. Jockers and J. Louis, "D-terms and F-terms from D7-brane fluxes," Nucl. Phys. B718 (2005) 203-246, hep-th/0502059.

[57] E. Witten, "Toroidal compactification without vector structure," JHEP 02 (1998) 006, hep-th/9712028.

[58] C. Bachas, M. Bianchi, R. Blumenhagen, D. Lüst, and T. Weigand, "Comments on Orientifolds without Vector Structure," JHEP 08 (2008) 016, 0805.3696 .

[59] I. Pesando, "A comment on discrete Kalb-Ramond field on orientifold and rank reduction," Phys. Lett. B668 (2008) 324-330, 0804.3931.

[60] A. M. Uranga, "D-brane probes, RR tadpole cancellation and K-theory charge," Nucl. Phys. B598 (2001) 225-246, hep-th/0011048.

[61] R. Blumenhagen, S. Moster, and E. Plauschinn, "Moduli Stabilisation versus Chirality for MSSM like Type IIB Orientifolds," JHEP 01 (2008) 058, 0711.3389 .

[62] S. H. Katz and E. Sharpe, "D-branes, open string vertex operators, and Ext groups," Adv. Theor. Math. Phys. 6 (2003) 979-1030, hep-th/0208104.

[63] T. W. Grimm and J. Louis, "The effective action of type IIA Calabi-Yau orientifolds," Nucl. Phys. B718 (2005) 153-202, hep-th/0412277.

[64] M. Cvetič, R. Richter, and T. Weigand, "Computation of D-brane instanton induced superpotential couplings - Majorana masses from string theory," Phys. Rev. D76 (2007) 086002, hep-th/0703028.

[65] L. E. Ibanez, A. N. Schellekens, and A. M. Uranga, "Instanton Induced Neutrino Majorana Masses in CFT Orientifolds with MSSM-like spectra," JHEP 06 (2007) 011, 0704.1079.

[66] M. Cicoli, J. P. Conlon, and F. Quevedo, "General Analysis of LARGE Volume Scenarios with String Loop Moduli Stabilisation," 0805.1029.

[67] M. Demazure, Séminaire sur les singularités des surface, ch. Surfaces de Del Pezzo, II, III, IV et V. Springer-Verlag, 1980. 
[68] K. Kodaira, "On compact analytic surfaces II," Annals of Math. 77 (1963) 563-626.

[69] K. Kodaira, "On compact analytic surfaces III," Annals of Math. 78 (1963) $1-40$.

[70] A. Klemm, B. Lian, S. S. Roan, and S.-T. Yau, "Calabi-Yau fourfolds for Mand F-theory compactifications," Nucl. Phys. B518 (1998) 515-574, hep-th/9701023.

[71] D. R. Morrison and C. Vafa, "Compactifications of F-Theory on Calabi-Yau Threefolds - II," Nucl. Phys. B476 (1996) 437-469, hep-th/9603161.

[72] J. Louis, J. Sonnenschein, S. Theisen, and S. Yankielowicz, "Non-perturbative properties of heterotic string vacua compactified on K3 x T**2," Nucl. Phys. B480 (1996) 185-212, hep-th/9606049.

[73] W. Barth, C. Peters, and A. Van den Ven, "Compact Complex Surfaces," Springer-Verlag, Berlin (1984).

[74] D.-E. Diaconescu, R. Donagi, and B. Florea, "Metastable quivers in string compactifications," Nucl. Phys. B774 (2007) 102-126, hep-th/0701104.

[75] M. R. Douglas and G. W. Moore, "D-branes, Quivers, and ALE Instantons," hep-th/9603167.

[76] G. Aldazabal, L. E. Ibanez, F. Quevedo, and A. M. Uranga, "D-branes at singularities: A bottom-up approach to the string embedding of the standard model," JHEP 08 (2000) 002, hep-th/0005067.

[77] J. P. Conlon, A. Maharana, and F. Quevedo, "Towards Realistic String Vacua," 0810.5660.

[78] R. Blumenhagen, M. Cvetič, R. Richter, and T. Weigand, "Lifting D-Instanton Zero Modes by Recombination and Background Fluxes," JHEP 10 (2007) 098, 0708.0403 .

[79] J. P. Conlon, S. S. Abdussalam, F. Quevedo, and K. Suruliz, "Soft SUSY breaking terms for chiral matter in IIB string compactifications," JHEP 01 (2007) 032, hep-th/0610129. 
[80] I. Garcia-Etxebarria and A. M. Uranga, "From F/M-theory to K-theory and back," JHEP 02 (2006) 008, hep-th/0510073.

[81] Y. I. Manin, "Rational surfaces over perfect fields. II," Mat. Sb. (N.S.) 72 (1967) 161-192.

[82] I. V. Dolgachev and V. A. Iskovskikh, "Finite subgroups of the plane Cremona group," math/0610595.

[83] L. Bayle and A. Beauville, "Birational involutions of $P^{2}$," math/9907028.

[84] T. de Fernex, "On planar Cremona maps of prime order," NAGOYA MATH.J. 174 (2004) 1, math/0302175.

[85] V. Braun, Y.-H. He, B. A. Ovrut, and T. Pantev, "Vector bundle extensions, sheaf cohomology, and the heterotic standard model," Adv. Theor. Math. Phys. 10 (2006) 4, hep-th/0505041. 